\title{
DESIGN OF A LOW PROFILE CONFORMAL ARRAY FOR TRANSCRANIAL ULTRASOUND IMAGING
}

\author{
AREF SMILEY \\ Master of Science in Biomedical Engineering \\ Cleveland State University
}

August 2014

\begin{abstract}
submitted in partial fulfillment of requirements for the degree of DOCTORAL OF ENGINEERING IN BIOMEDICAL ENGINEERING

at the

CLEVELAND STATE UNIVERSITY
\end{abstract}

May 2018 


\section{ACKNOWLEDGMENTS}

Thanks to God for all the blessings he has given me in my life.

I would like to thank my adviser Dr. Greg Clement for his unconditional support, guidance, encouragement, and patience throughout my $\mathrm{PhD}$ program. Without his support this project would not have happened. Upon his departure from the lab, Dr. Aaron Fleischman took over in an advisory role and I must thank him for bringing added detail and closure to my project.

Dr. Margot Damaser took a chance on me by hiring me to work in her lab while I was still finishing this project, and I could not be more grateful that she did. She was very supportive and understanding about my obligations and gave me a great opportunity to succeed in the next chapter of my life.

I would also like to thank my academic adviser, Dr. Dan Simon and his wife, Annette for their support and encouragement. While I was trying hard to succeed in my program, I faced many challenges. Dr. Simon was always there for me as an instructor, mentor, and confidant. I could not overcome my challenges without his support.

I would like to thank all of my thesis committee members.

During my program, I had an opportunity to work at Cleveland Clinic, Dr.

Clement ultrasound lab. The lab manager, Mark Howell, had pivotal role in my success. I would like to thank him for all he has done for me. He is a great friend and a lab partner.

Last but not least, I would like to thank my family for their unconditional support throughout my time at CSU. In particular, my mum, Tooran, my dad, Ali and my dear brother, Dr. Abbas Smiley. 


\title{
DESIGN OF A LOW PROFILE CONFORMAL ARRAY FOR TRANSCRANIAL ULTRASOUND IMAGING
}

\begin{abstract}
AREF SMILEY
ABSTRACT

A "belt-type" conformal transducer array designed transcranial brain imaging has been investigated. In precursory simulation work it was estimated that an array consisting of approximately 500 channels would be sufficient to achieve two-dimensional brain imaging utilizing a tomographic approach valid on irregular boundaries [1], such as the human head. To achieve such an array in practice a transducer designed and constructed around an assembly of rigid 1-3 composite planar sub-arrays $(500 \mathrm{kHz}, 1-3$ random-fiber, $20 \mathrm{~mm}$ X 20mm) consisting of 19 elements. Sub-arrays are bonded to custom-printed circuit boards wired to shielded ribbon cable. The full transducer is created by UV bonding the electrodes to a flexible 3D-printed head band. Element performance is assessed by underwater scanned hydrophone characterization in the far-field. Measurements are then numerically back-projected to the transducer face to assess isolation. It is determined that elements function independently with little impact on the nearby elements. The maximum measured pressure amplitude crosstalk of the neighboring elements to the active element is less than $-14 \mathrm{~dB}$, which satisfies the later tomography imaging requirements. Results also show a maximum $3 \mathrm{~dB}$ decrease in the pressure amplitude as referenced to the maximum pressure amplitude of elements. Due to the limited number of channels on the data and control acquisition system $(\mathrm{N}=128)$, an expander was designed and fabricated using 4:1 multiplexers (MUXs) to cover all the required number of transducer elements for the imaging system. The electrical characterization of the expander was addressed in terms
\end{abstract}


of noise and crosstalk. Results show ae crosstalk of about $-58.7 \pm 2.9 \mathrm{~dB}$ for the MUXs. Average signal to noise ratio (SNR) decreased by about $0.05 \mathrm{~dB}$ after adding the expander. Noise level remained almost the same after increasing the average detected signal power. Overall, the design and measured results are found to satisfy the key transducer requirements for a conformal array designed for transcranial diffraction tomography. This "work in progress" will be combined with an optical registration technique to form a complete imaging device. 


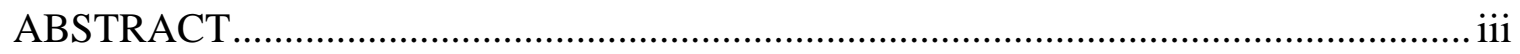

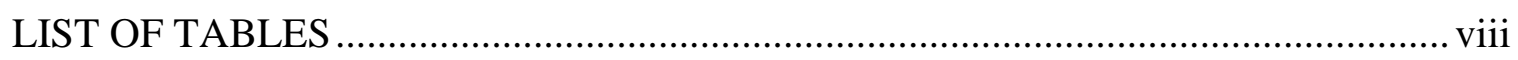

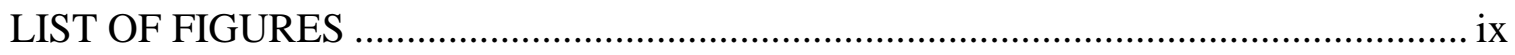

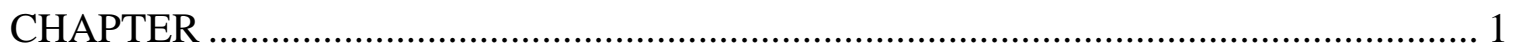

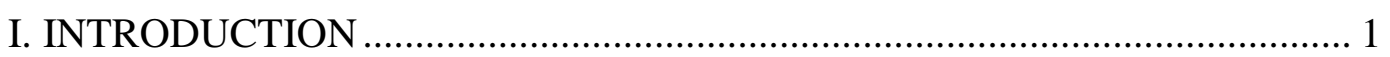

1.1 Problem Overview ......................................................... 1

1.2 Literature Review .............................................................. 6

1.3 Motivation for Fabricating the Transducer Array ......................... 9

1.4 Thesis Organization and Contribution..................................... 12

II. BASICS OF ULTRASOUND ................................................................ 15

2.1 The Ultrasound ................................................................... 15

2.2 What is Ultrasound Transducer? .............................................. 16

2.3 Ultrasonic Transducer Array ................................................. 23

2.4 Ultrasound Pressure Waves ................................................... 24

2.4.1 Ultrasound Wave Properties ....................................... 26

2.4.2 Beam Properties ..................................................... 27

2.5 Interaction of the Ultrasound with Body Tissues ......................... 34

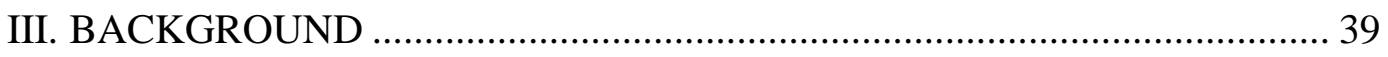


3.1 Transcranial Ultrasound

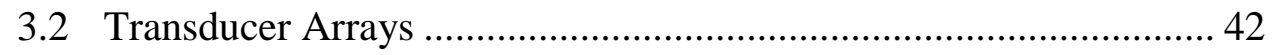

3.3 Image Reconstruction Algorithms ........................................ 45

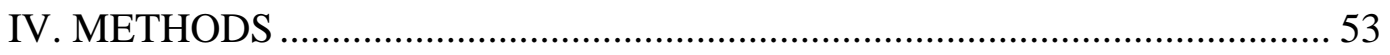

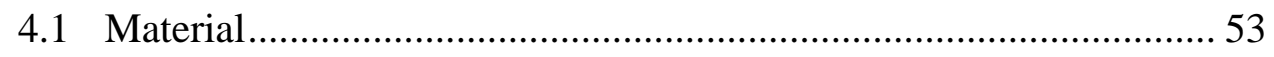

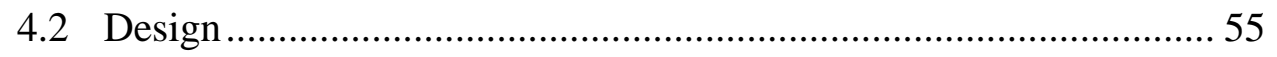

4.2.1 Transducer ............................................................. 56

4.2.2 PC Board .................................................................. 57

4.2.3 Connecting PC Board to PZT ........................................ 59

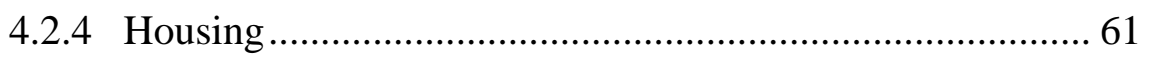

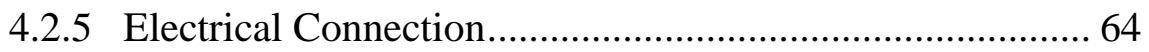

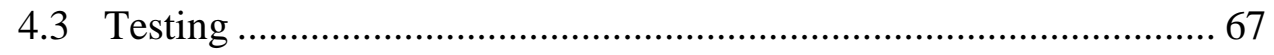

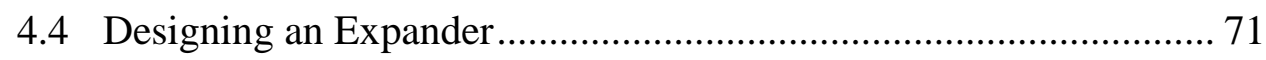

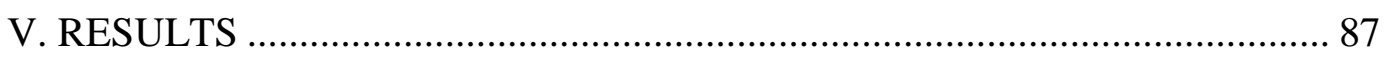

5.1 Transducer Functionality .................................................. 87

5.2 Expander Characteristics .................................................. 90

VI. CONCLUSION AND FUTURE WORK ................................................ 103

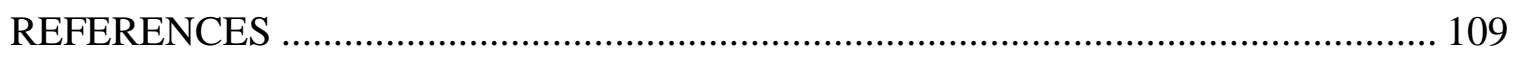

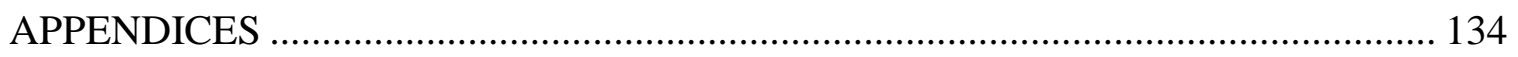

A. Silver Conductive Epoxy Data Sheet .......................................... 135 
B. Low Viscosity, "Wicking” Epoxy Data Sheet ................................... 137

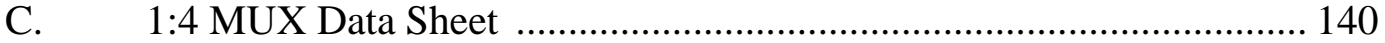




\section{LIST OF TABLES}

Table

Page

I. Importance of transducer frequency in clinical ultrasound applications ................. 27 


\section{LIST OF FIGURES}

Figure

1. A concept of how the array transducers device ................................................... 10

2. Ultrasound imaging system ……………………...................................... 12

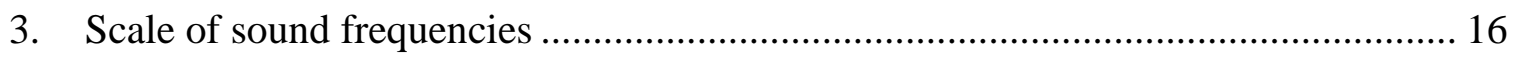

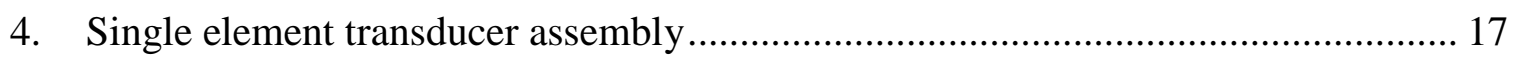

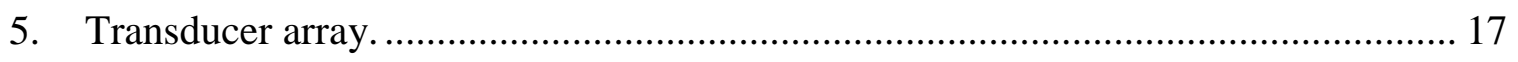

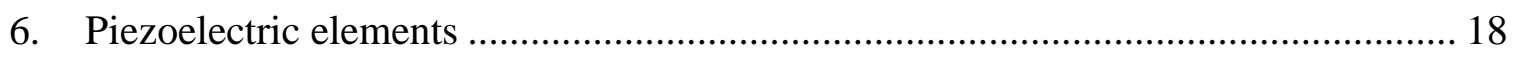

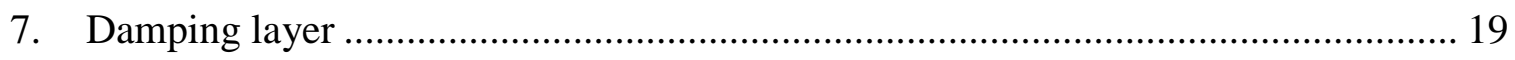

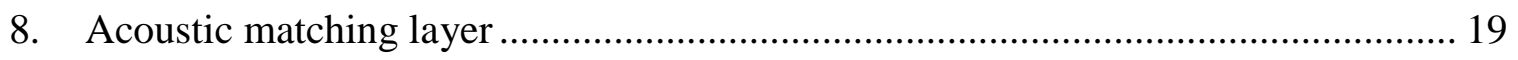

9. Optimal size of the matching layer........................................................................ 20

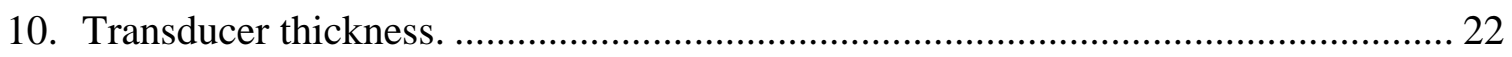

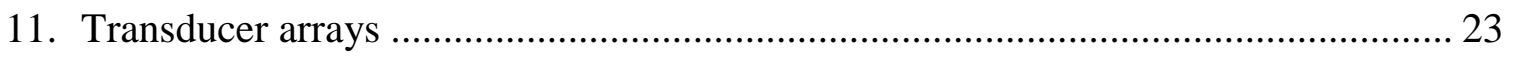

12. Propagation of ultrasound through a medium ……….............................................. 24

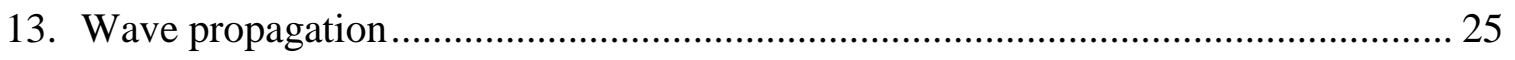

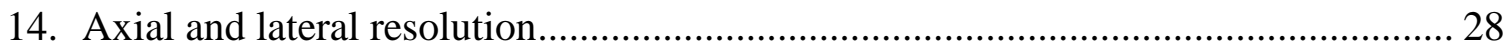

15. Schematic of a typical ultrasound transducer ........................................................ 32

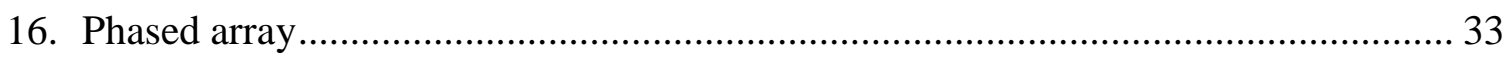

17. Adjustable focal zone in phased-array transducers ................................................... 34

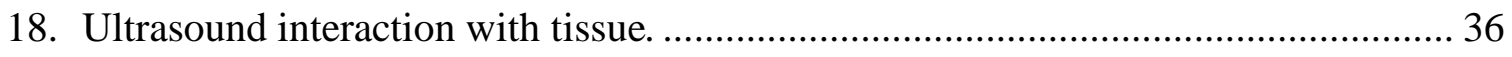

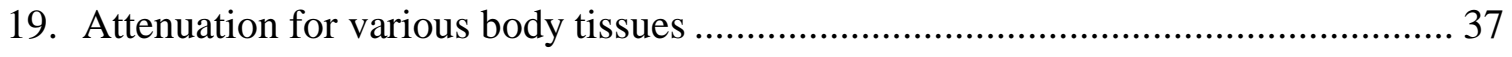

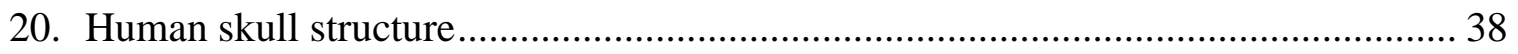

21. MR guided focused ultrasound, insightec. ............................................................ 42 


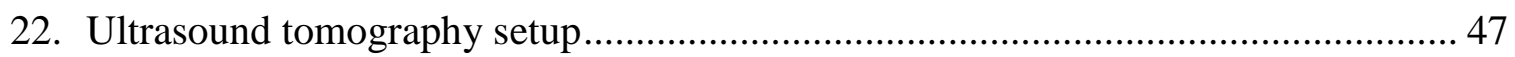

23. Magnetic resonance imaging (MRI)..................................................................... 49

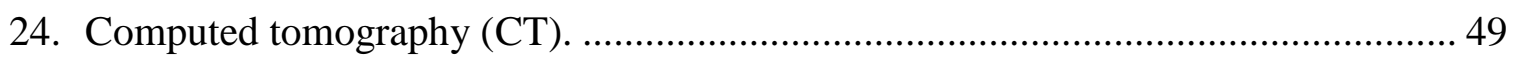

25. A graphic showing how PZT transducers are created ............................................ 55

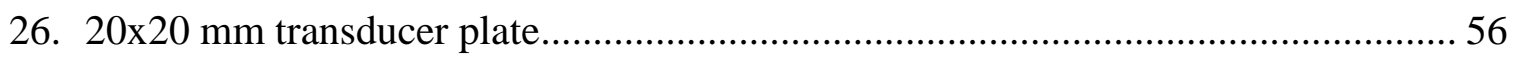

27. Different views of the PC board with connectors attached to it ............................... 58

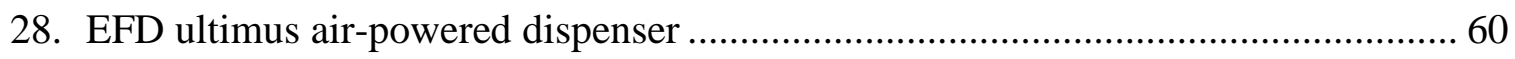

29. Conductive epoxy on the PZT before connecting the PZT to PC board ................... 60

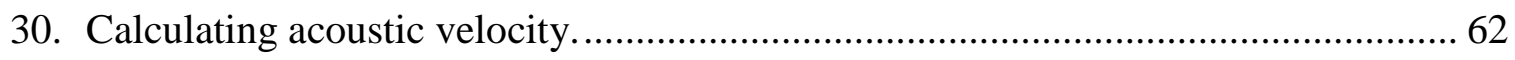

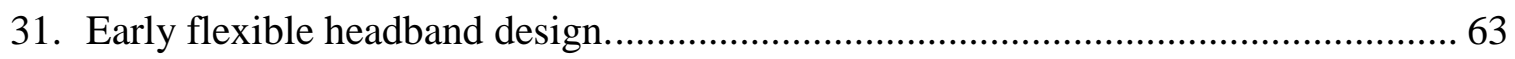

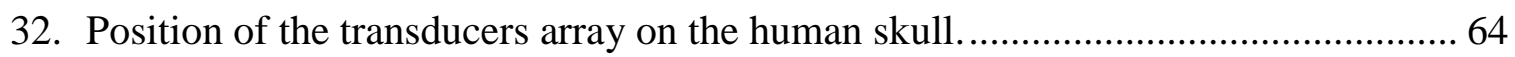

33. Electrical connection of a sample of a transducer plate connected to PCB .............. 64

34. Array interface view of the control and acquisition system..................................... 65

35. A male plug header with contacts sticking out of the back of it .............................. 65

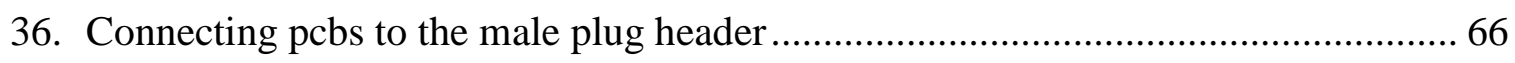

37. Experimental setup for measuring the pressure plane ............................................. 67

38. Example of pressure amplitude and phase information............................................. 70

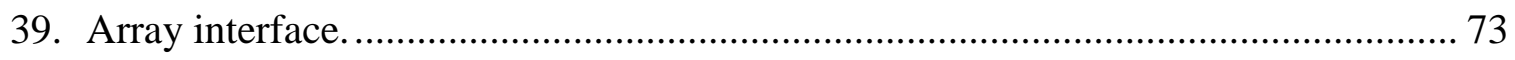

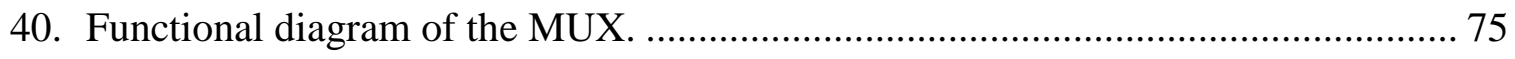

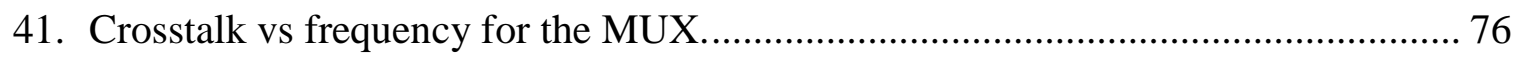

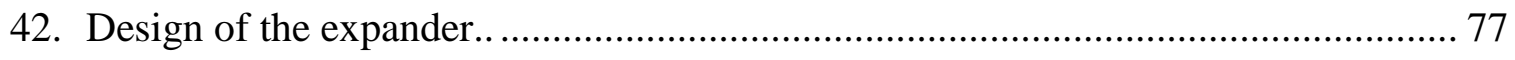

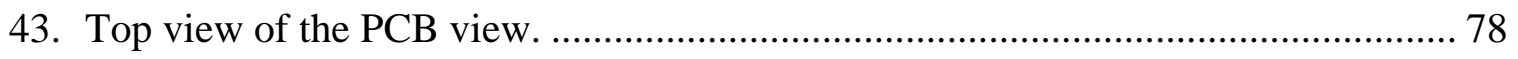

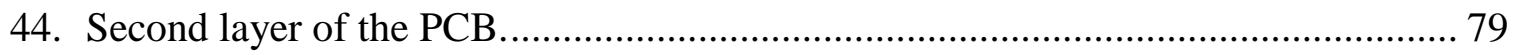




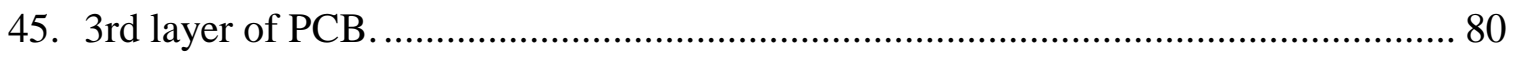

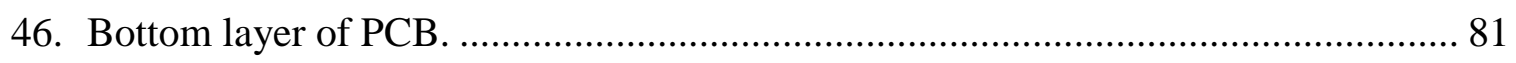

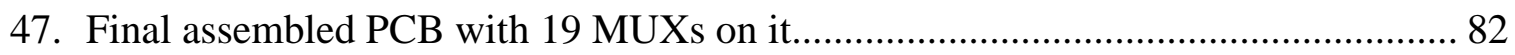

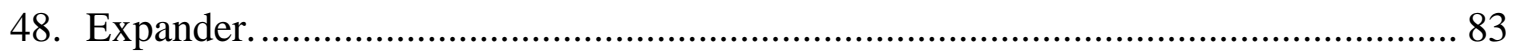

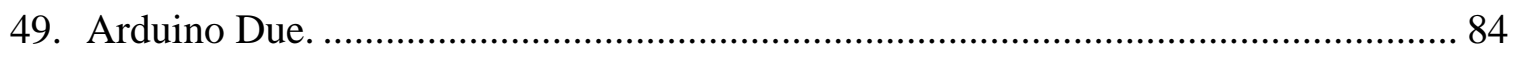

50. Switch between the channels of the MUX in which the signal is firing. .................. 85

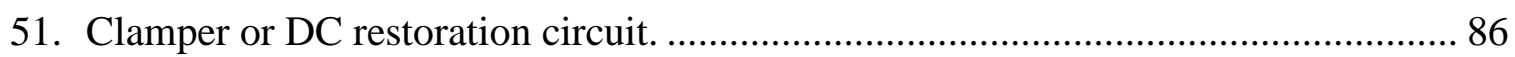

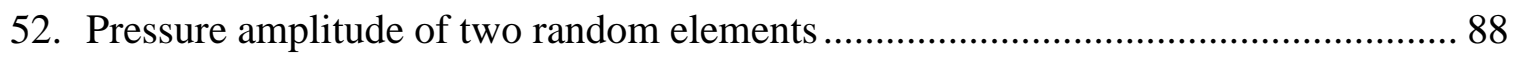

53. Pressure amplitude of two elements located at very edges of transducer. ................ 89

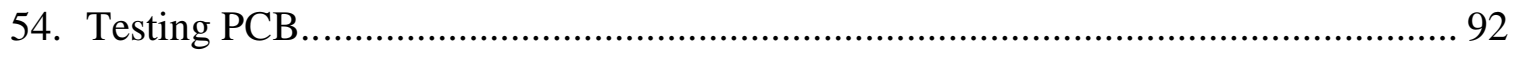

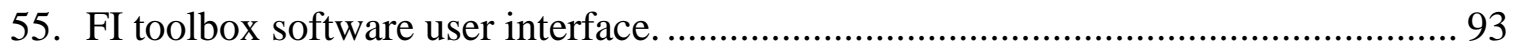

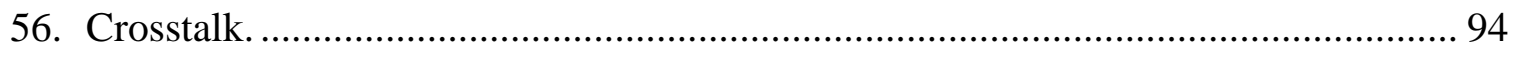

57. Histograms of SNR for 50 collected measurements with and without exopander.... 95

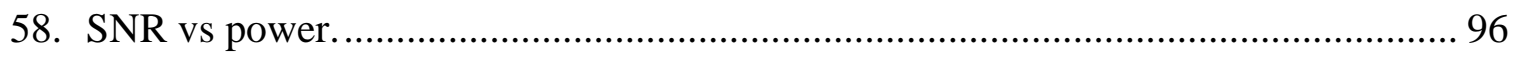

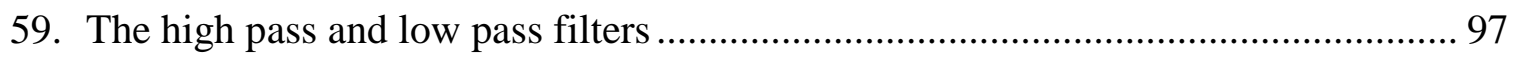

60. Frequency response of a functioning transducer element......................................... 98

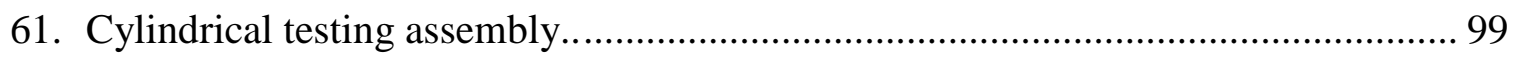

62. Connecting transducers to cylindrical testing assembly ....................................... 100

63. Received scattered ultrasonic signals using the array........................................... 101

64. Using 3D scanner to find the location of each transducer...................................... 107

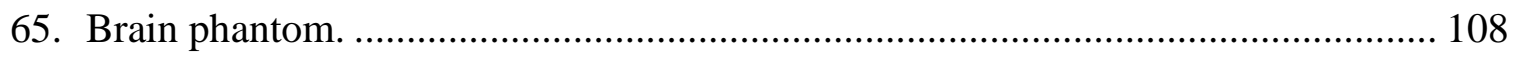




\section{CHAPTER I}

\section{INTRODUCTION}

Section 1.1 gives the problem overview. Section 1.2 provides a literature review related to the topics discussed in this thesis. Section 1.3 gives a discussion of the motivation for brain imaging by using ultrasound. Section 1.4 summarizes the contributions of this thesis.

\subsection{Problem Overview}

Numerous studies have demonstrated the potential use of transcranial ultrasound (US) in brain therapies, including tumor ablation, stroke, and localized opening of the blood brain barrier[2]-[15]. The hope is to apply the noninvasive US through the intact skull [16], [17]. However, there are some major problems for ultrasound passing through the bone, which attenuates and scatters the signal [18]. These problems make the transcranial ultrasound propagation a major issue if noninvasive procedures are to become common practice. Prior to more contemporary achievements in transcranial focusing, animal experiments where skull sections were surgically removed had shown 
that focused ultrasound could be applied to open the blood-brain barrier [19], activate drugs [20], and close blood vessels [21]. However, the need for such a surgical procedure was a deterrent to applying ultrasound to the brain clinically.

Such problems can be overcome by transmitting ultrasound energy through the skull without surgery. The procedure is achievable by the means of an ultrasound transducer array to offset skull distortion and focusing ultrasound inside the brain without problematic heating at the brain-skull interface [4].

Non-invasive ablation of tissue inside the brain by using an array of large number of transducers is already tested and approved by food and drug administration (FDA) [22] for the treatment of essential tremor. By using low frequency with high intensity ultrasound waves, they could ablate the tissue at focal point inside the tissue.

Due to the limitations involved in passing the ultrasound energy inside the skull, non-invasive imaging of the brain has not been the topic of interest for researchers. Compare to soft tissue, the skull has variable and highly contrasting acoustic properties which causes severe phase aberrations. Moreover, signal propagation would be impeded due to the high attenuation caused by the skull. For more penetration, lower frequencies are required. However, the use of lower frequencies is generally a tradeoff between depth penetration and radial resolution [23]. This is explained in detail in Chapter 2.

Due to the structural nature of the human skull bone, it attenuates the ultrasound wave energy by about $90 \%$ even though low frequency ultrasound $(<1 \mathrm{MHz})$ is used (see Chapter 2 for more details). This means after first penetrating the skull and then being received after passing a second time back through the skull, the signal strength has been reduced by about $99 \%$. 
Therefore, non-invasive imaging of the brain requires maximizing the energy propagated into the cranial vault and the ability to collect the very small signals that are scattered out of the skull, in order to reconstruct an image.

A new concept of diffraction tomography investigated by G. T. Clement [1] shows that it is possible to construct a $2 \mathrm{D}$ slice image of any curved shape plane by surrounding the plane with ultrasound transmitters and receivers. This tomographic ultrasound technique, verified in a simulation study, is a single-frequency phase-based imaging approach which brings about two important features for brain imaging: 1- single-frequency imaging allow us to send more energy inside the skull at that frequency (continuous wave signals are used) and 2- it also enhances receivers sensitivity for measuring the weak scattered signal at that frequency (more sensitive narrow-band receivers can be used). These features are important because of the fact that the strength of even directly-received signals are around $1 \%$ of the transmitted signals after passing the human skull [24]-[26] (see Chapter 2).

Ultrasound diffraction tomography has been under consideration researches in medical imaging field since the mid-1980s. Ultrasound diffraction tomography is a technique for imaging based on mapping acoustic properties, including attenuation, density, and etc. such properties by collecting the complex pressure (amplitude and phase) of scattered waves resulting from a known transmission source. Most commonly this is a transmitted plane wave or point-source at a single temporal frequency [27].

In medical applications, as the acoustic properties of normal and diseased tissues have different values, ultrasound diffraction tomography is capable of providing functional information of the object of interest. For example, in breast cancer imaging, 
the benign mass, the malignant tumor, and the normal tissue can be differentiated from each other [28], [29].

Fourier diffraction theory is central to the image reconstruction in diffraction tomography [30], [31]. It works under the assumption of weak scattering constituting a Born or Rytov approximation [32]. In the case of plane wave tomography, the object of interest is illuminated by a plane wave from a certain direction and the scattering waves are collected along a planar receiver. Then the spatial Fourier transform would be applied over the received data., the 1-D transform values are then mapped onto a 2D surface, representing the values of the Fourier transform of the object itself along a semicircle arc. The process would be repeated after rotating the transmitter and receiver at incremental angles around the object until sufficient spatial frequency information is available to provide an inverse transform. Finally, an ultrasound diffraction tomography image is reconstructed through inverse of this $2 \mathrm{D}$ mapped spatial Fourier transform. The process can be repeated with more spatial frequency samples to avoid under-sampling, but this repetition increases the scan and process time [29].

Chapter 3 provides more details of how the data is collected in diffraction tomography. In order to image an object through the presently-proposed diffraction tomographic approach, the object needs to be surrounded with transmitters and receivers over a plane. This could be done by designing an array of transducers which surrounds the object.

One of the biggest limitations of ultrasound diffraction tomography is that it is only applicable for either symmetric geometries or on a curvature with restrictions [33], [34]. These limitations prevent the ultrasound to be applied for the structures with 
irregularly-shaped boundaries where there is need to couple to those structures. For example, the human head is both structurally non-symmetric and highly attenuates the signal. Without providing coupling of the transducers to the skull skin, it is impossible to penetrate the ultrasound through the skull and then collect the scattered signal. On the other hand, coupling the transducer to the human head creates nonsymmetrical geometry. Therefore, in addition to applying low frequency ultrasound for more penetration, it is necessary to tightly attach the transducers to the human head as any gap between the transducer and the skull would cause a huge reflection and contribute to a further loss of energy. This requires an image reconstruction algorithm applicable to irregularly-shaped boundaries of the imaged plane, like human skull.

The new concept of diffraction tomography [1] evaluates 2D image reconstruction of objects which are surrounded by an arbitrarily-shaped curve of sources and receivers. The approach does not require explicit restrictions on curvature. This method was tested on three different irregular curves, in addition to a Shepp-Logan phantom, by using two virtual source/receiver pairs at $0.5 \mathrm{MHz}$ frequency (a sound speed similar to the range of many fluids and tissues). The results show that point-scatters inside the surrounding elements could be identified with low distortion, but with little image contrast [1]. The new technique was developed to reconstruct the image around irregularly-shaped boundaries and may be applied in any boundary shapes, especially in the situations where long boundary needs to be covered by sources and receivers to reconstruct an image.

Therefore, in order to reconstruct the brain image noninvasively by applying the new ultrasound diffraction tomography approach, a conformal array of transducers is needed to be coupled around the head with high sensitivity to its resonance frequency to 
collect the weak scattered signals. Because of the limitations of applying ultrasound to human skull as well as lack of an appropriate image reconstruction algorithm for the intended application, there is no reported such an array to be made by the date of writing this dissertation. The central part of my $\mathrm{PhD}$ dissertation is to design, fabricate, and test a conformal array capable of taking 2D images of human brain noninvasively.

\subsection{Literature Review}

Safe, low-cost, and portable ultrasound imaging has become widespread in clinical radiology. However, it is restricted to use only in certain areas of the body. Using ultrasound for imaging in the skull is hindered by the steep challenge of beam focusing. Compared to skin and brain tissue, the skull has variable and highly contrasting acoustic properties, causing severe phase aberrations. This can harm the tissue outside the region of interest (ROI) [18], [35], [36]. Acoustic phase is known as the main factor of determining the ultrasound focus [37], [38]. The feasibility of ultrasound focus reconstruction after passing human skull was initially shown by manual adjustment of a transducer array's driving phases [17]. However, the phased signal should only have significant acoustic pressure at the ultrasound focus and not too much in other regions, like the skull surface. There is also an issue of accurately focusing ultrasound in the brain without overheating the skull, but this has been overcome by the development of phased arrays [18], [35], [39]. Different identifying methods to show how to estimate phase distortions in vivo were investigated [40]. In most of the methods, estimation of the structural and acoustic properties of the skull was achieved from computed tomography (CT) scans of the head [41]-[43]. 
One of the most important factors in signal propagation through the skull is frequency. Studies have shown that signal propagation is impeded due to high attenuation caused by the skull, specifically at frequencies above $1 \mathrm{MHz}$, as the absorption coefficient increases rapidly with frequency [44]-[46]. The possibility of focusing ultrasound through the skull with less distortion has been investigated for frequencies less than $1 \mathrm{MHz}$ [13], [16], [26]. These studies showed the feasibility of focusing, but also that distortion would be inevitable [2]. Investigations on the heat produced at the surface of the skull showed that the optimal thermal gain between the focus in brain region of interest (ROI) and the skull surface would have an average frequency of $700 \mathrm{KHz}$ [4], [21], [23], [40]-[42], [47]-[51]. G. T. Clement [40] showed that the change in temperature, caused by phase distortion, would be less than $14^{\circ}$ in the skull at $0.51 \mathrm{MHz}$ when the experiment temperature was varied between $22^{\circ} \mathrm{C}$ and $50^{\circ} \mathrm{C}$. This means that phase correction could be applied through a skull at room temperature. It was also concluded that the increasing temperature in the skull bone (which can happen during a therapeutic US treatment) should not significantly change the phase [40].

Frequency is also important in determining resolution in ultrasound imaging since higher frequencies are capable of producing higher resolution. On the other hand, high frequencies cause more absorption. Therefore, finding a clinically relevant resolution while having enough signal strength becomes problematic for highly absorbing structures. Such an issue is very relevant to applications in transcranial imaging, where there is a trade-off between the resolution and signal penetration. More penetration needs lower frequencies and therefore, more resolution reduction [23]. 
Backscattered signal strength is another key issue. Even in the relatively low frequency range of $0.5 \mathrm{MHz}$ to $1 \mathrm{MHz}$ only about $10 \%$ of an acoustic signal (power) is transmitted through the skull [24]-[26]. Therefore, in a pulse-echo scenario signals on the order of $1 \%$ of its soft-tissue equivalent would be expected.

An approach that could overcome issues of limited signal strength and image resolution is the use of single-frequency diffraction tomography [1] in transmission mode, which uses an array that surrounds the region to be imaged and utilizes phase information from both forward and backscattered signals. This approach was considered in a simulation study [1], which examined diffraction tomography for cases of irregular geometry such as the human head. It was concluded in this work that a $0.5 \mathrm{MHz}$ array with source/receiver locations separated by approximately $0.97 \mathrm{~mm}$ intervals along a closed curve could achieve a resolution of approximately $1.3 \mathrm{~mm}$ (around half of the wavelength), indicating that an array of up to 512 elements is necessary to accommodate an adult head.

In the present work, a transducer that is designed to satisfy all key acoustic requirements of [1], yet also remain feasible in terms of practical constrains of usability and manufacturability, is investigated. A flexible, circumferential belt shape design is described that conforms to the head such that it can be coupled to the skin while remaining sufficiently lightweight that it may be worn without slipping and without external support. The design is based upon the linking of 30 rigid planar sub-arrays. These sub-arrays were further divided into 19 sending/receiving elements to form a flexible array of transducers operating at resonance frequencies of $0.5 \mathrm{M} \mathrm{Hz}$. In our 
design, the sub-arrays could be added/dispatched to/from the array to fully surround the skull depending on the size of the human skull.

To test the experimental feasibility of the transducer, a planar area of the ultrasound was measured in a water tank at specific distance in front of the transducer. Then the acoustic phase and amplitude was determined by using temporal Fourier transform of the received waveform at the transducer's driving frequency. Finally, the planes will be back-propagated to the surface of the transducer to see the influence of the element and its surrounding elements on the phase and amplitude properties.

Results show that each element is finely separated from each other and there is less than $3 \mathrm{~dB}$ decrease in the pressure amplitude of different elements compare to the biggest one.

\subsection{Motivation for Fabricating the Transducer Array}

The goal of this thesis is to fabricate of a transducer array capable of focusing ultrasound through the skull to acquire a $2 \mathrm{D}$ image. This will be done by a circumferential transducer array fastened in a belt shape around the head; as the aim is to later use the concept of diffraction tomography for imaging the brain [1], an array needs to be designed to cover the entire plane of interest to collect the signals. In the simulation example done in [1], it was concluded that when source/receiver locations are separated by around $0.97 \mathrm{~mm}$ intervals along the closed curve, spatial distortion would be negligible in ROF, the resolution of $1.3 \mathrm{~mm}$ (around half of the wavelength) or greater is distinguishable, and image artifacts are not seen. In order to meet this criteria, our conformal array will consist of around 30 individual transducers to fully surround the 
skull (Figure 1), each of which having 19 separate sending and receiving elements which operate at resonance frequencies of $0.5 \mathrm{MHz}$.

The array needs to be designed in a way that would minimize the gaps between transducers, use an optimal coupling lay to transmit sound waves from the transducers into the head, and stay light weight enough to be portable and comfortable to wear during scanning.

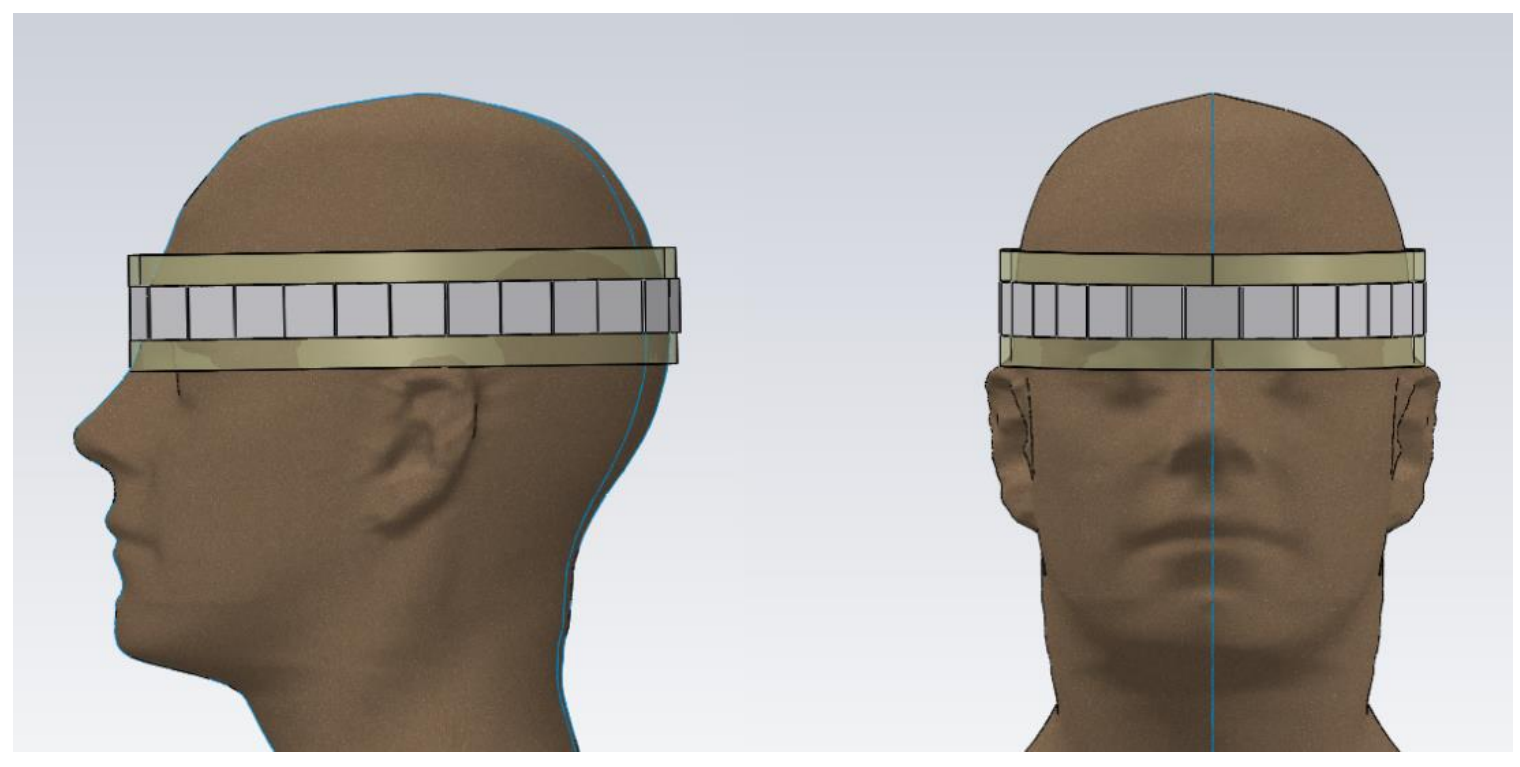

FIGURE 1: A CONCEPT OF HOW THE ARRAY TRANSDUCERS DEVICE WILL LOOK LIKE.

In order to test the experimental feasibility of the transducer, a planar area of the acoustic fields measured in a water tank at specific distance in front of test elements. Then the acoustic phase and amplitude will be determined by using temporal Fourier transform of the received waveform at the transducer's driving frequency. Finally, the planes will be back-propagated to the surface of the transducer to see the influence of the element and its surrounding elements on the phase and amplitude properties (see Chapter 4, Section 4.3). 
The way transducers are fabricated is unique as micro-scale conductive epoxy is used to connect 1-3 PZT composite transducers to PC boards without further backing. This would cause a narrower bandwidth of frequencies around our desired frequency. As the intended imaging technique [1] works with single frequency to deliver more energy as well as to enhance receivers sensitivity for that frequency, transducers need to be designed the with highest sensitivity to the resonance frequency.

In addition, for providing optimal coupling lay as well as housing the transducer near each other with minimum possible gaps to form a flexible array, optimal thickness calculated and tested for the a rectangular shaped 3D printed soft materials to later attach to the surface of the transducers (See details in Chapter 4). This will be done by using liquid version of the material between the transducer surface and the belt. As will be shown in more details in Chapter 4, by applying UV light, not only a strong bondage of the transducers and the belt can be achieved, but also flexible matching layer to easily wrap it around the head.

Designing of a new ultrasound imaging system for the brain would provide major advantages over other image modalities. First, compare to CT and MRI, ultrasound imaging systems are portable. It is especially important for rural areas where accessibility of other image modalities are very limited. Second, compare to CT and MRI, ultrasonic imaging system are much cheaper. Third, ultrasound provide real time monitoring of the image (depending on the image reconstruction algorithm); and so it can provide early detection of problems, especially when the resolution of the image is not that important and having access to other image modalities is limited. For example, the imaging system can be used in the ambulance to detect the brain bleeding (hemorrhages or cerebral 
hemorrhages) which mostly happened during the injury. Figure 2 shows the potential setup of the ultrasonic based imaging system in the future for getting 2D plane images of the brain.
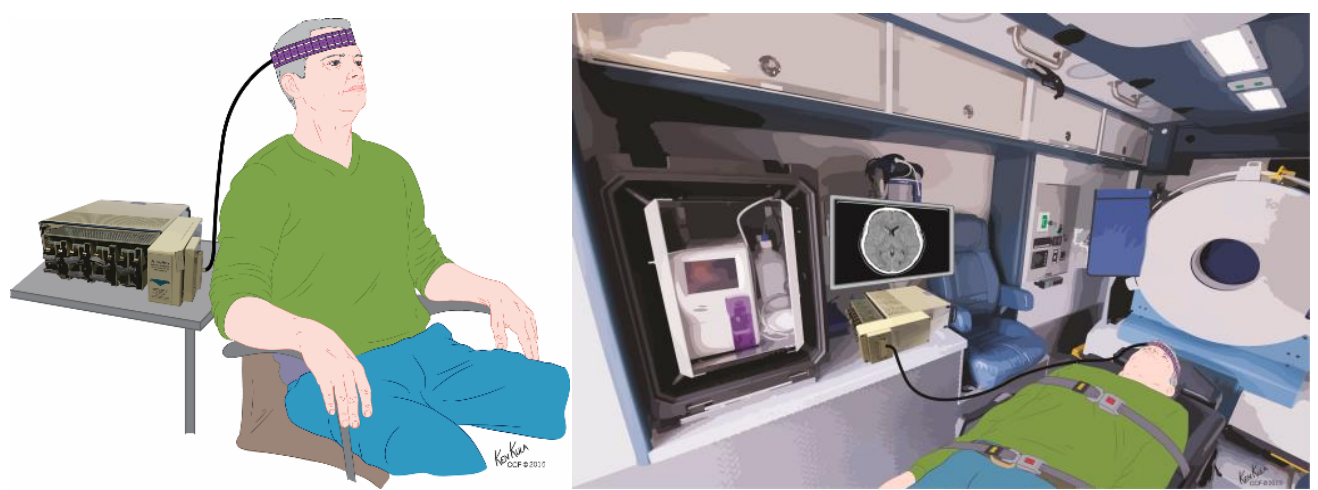

FigURE 2: ULTRASOUND IMAGING SYSTEM. PORTABILITY, LOW COST, AND ITS CAPABILITY TO GET REAL TIME IMAGES OUTWEIGH THE OTHER IMAGING SYSTEMS WHEN THE IMMEDIATE DIAGNOSIS PLAYS AN IMPORTANT ROLE. THE IMAGE SHOWS THE FUTURE HOPE OF USING OF ULTRASONIC SYSTEM IN AMBULANCES.

As it is shown in Figure 2, the transducers array would be designed in a way that it tights around the head to provide $2 \mathrm{D}$ image of the surrounded plane. Therefore, one of the biggest advantages of the system is that by moving the patient there would be no image artifacts in the final image. The array is designed in a way that it could be adjusted to different size of the human skulls. Small pieces of transducers would be added for bigger skull sizes and vice versa. In the next section, some of the requirements and challenges that address specifications that should be considered for designing the system are reviewed. These requirements are addressed in previous publications and researches.

\subsection{Thesis Organization and Contribution}

Chapter 2 provides basics of ultrasound. This chapter start with defining of the ultrasound (Section 2.1) and the ultrasound transducer (Section 2.2). Section 2.2 also talks about the basic elements of the transducers as well as their functions. Section 2.3 evaluates the Ultrasonic Transducer array. Ultrasound pressure waves are discussed in Section 2.4. 
This section includes two subsections: Ultrasound pressure waves (2.4.1) and Beam Properties (2.4.2). Finally, the interaction of the ultrasound with body tissue is evaluated at Section 2.5.

Chapter 3 presents a summary of similar works that have been done in transcranial ultrasound. As brain imaging is completely a new concept, there is no previous work to be considered as the background. Due to the similar limitations of the focused ultrasound to our project, background of the transcranial ultrasound has been reviewed in Section 3.1. This would be followed by a brief overview of the transducers arrays in Section 3.2. These arrays have been made for different purposes. In this section, the important features of the design are addressed in previous projects although there is no array of transducers was developed for brain imaging purpose due to the limitations mentioned before. In Section 3.3, a brief background of the reconstruction algorithms is provided.

Chapter 4 illustrates a detailed review of our design process. This section starts with a discussion of the choice of materials (Section 4.1). It then followed by our design process in Section 4.2. This subsection talks about transducers preparation for our intended use (Section 4.2.1), PC board design to provide electrical connectivity to the PZT on one side and to the data acquisition system for data collection on the other side (Section 4.2.2), connecting PC board to PZT for further mechanical support (Section 4.2.3), and housing for putting transducers near each other and providing a flexible matching layer (Section 4.2.4). Section 4.2.5 talks about how and why it is decided to choose ribbon cables to electrically connect the transducers to our data acquisition system. 
Chapters 5 shows our results in evaluating the array and transducers function.

Isolation evaluation tests are explained in Section 5.1 and the results of the expander characterization are discussed in Section 5.2.

Finally, Chapter 6 discusses our conclusions, and future work. 


\section{CHAPTER II}

\section{BASICS OF ULTRASOUND}

This chapter provides information about the physics of ultrasound in addition to the basic requirements for designing transducers for intended use. Section 2.1 talks about the definition of the ultrasound. Section 2.2 provides information about different parts of a typical ultrasound transducers and their functioning. Section 2.3 and 2.4 discuss about the transducer arrays and ultrasonic beam propagation respectively. Finally, interaction of the ultrasound with body tissues is discussed in section 2.5 .

\subsection{The Ultrasound}

Ultrasound refers to acoustic or sound energy in the form of waves with frequency above the human detectable hearing range. Its applications include but are not limited to medical diagnostics, underwater, manufacturing, aerospace, navigation and nondestructive testing (NDT) and material characterization in the metallurgy, and automotive industries. With the audible sound frequency range being $20 \mathrm{~Hz}$ to $20 \mathrm{kHz}$, 
ultrasound is the sound waves with frequencies above $20 \mathrm{kHz}$. In diagnostic ultrasound, the frequency range between 2 and $20 \mathrm{MHz}$ is typically used to see internal body structures (Figure 3).

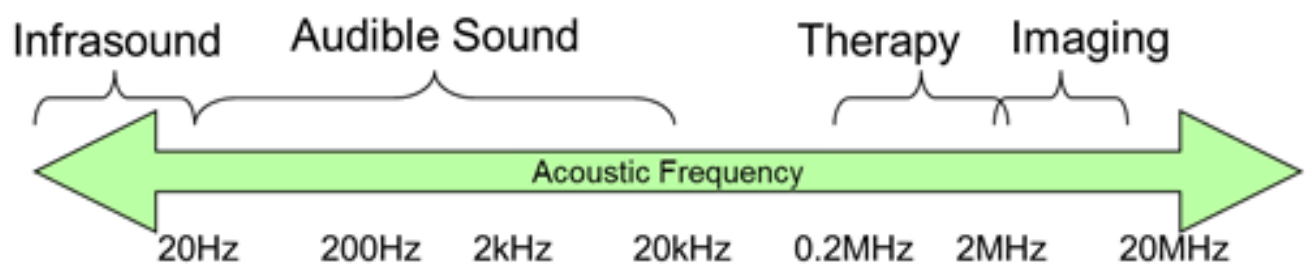

FIGURE 3: SCALE OF SOUND FREQUENCIES RANGING FROM $<20 \mathrm{~Hz}$ TO $>20 \mathrm{MHZ}$. TAKEN FROM [52].

\subsection{What is Ultrasound Transducer?}

Transducers are capable of producing and detecting ultrasound. The design and functionality of the transducer assembly has been changed during the last few decades, however. Such assemblies vary from single-element resonance crystals to a broadband transducer array of hundreds of individual elements. A schematic of a typical single element transducer as well as an array of transducers are shown in Figure 4 and 5. In general, all transducers have the same basic elements:

- piezoelectric material,

- matching layer,

- backing block,

- acoustic absorber,

- insulating cover, 
- $\quad$ sensor electrodes, and

- $\quad$ transducer housing

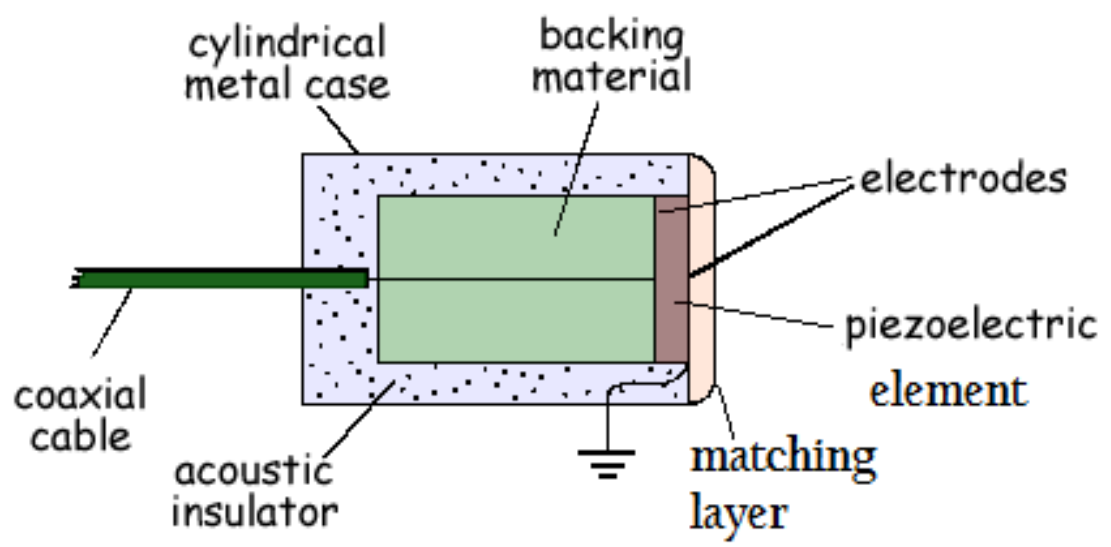

FIGURE 4: SINGLE ELEMENT TRANSDUCER ASSEMBLY COMPRISED OF A PIEZOELECTRIC ELEMENT, DAMPING LAYER, IMPEDANCE MATCHING LAYER, ACOUSTIC ABSORBER, INSULATING COVER, SENSOR ELECTRODES, AND TRANSDUCER HOUSING. TAKEN FROM [53].

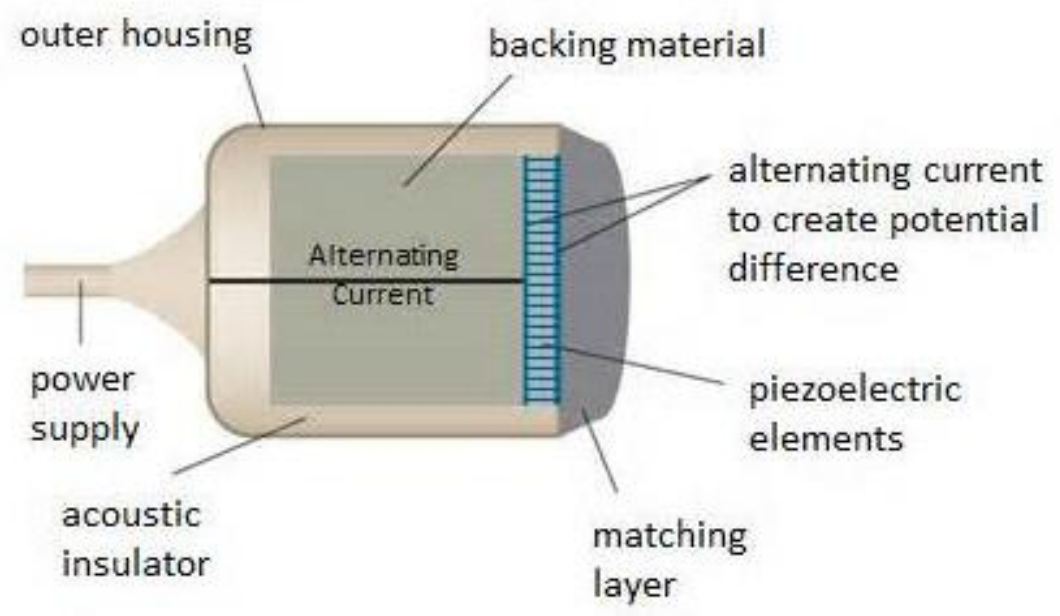

FIGURE 5: TRANSDUCER ARRAY. TAKEN FROM [54].

A typical transducer composed of one or more piezoelectric elements with electromechanical (piezoelectric) properties. In order to produce ultrasound, electrical 
energy would be converted into mechanical energy by the ceramic element. Conversely, for ultrasound detection, the ceramic element converts mechanical energy into electrical energy.

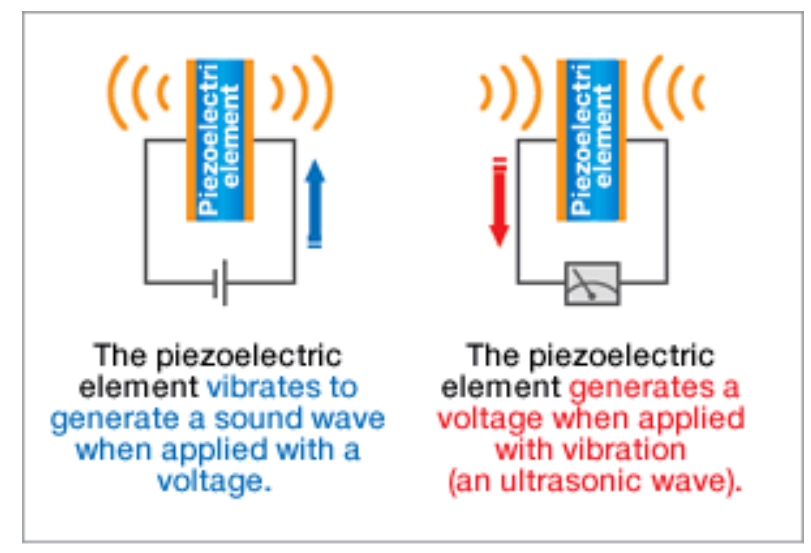

Figure 6: Piezoelectric elements Vibrate by RePeatedly expanding AND CONTRACTING WHEN A Voltage IS APPLIED, CREATING SOUND WAVES. CONVERSELY, MECHANICAL PRESSURE APPLIED TO ITS SURFACE, SUCH AS A SOUND WAVE, GENERATES A VOLTAGE PROPORTIONAL TO THE INCIDENT PRESSURE AMPLITUDE. TAKEN FROM [55].

The amplitude of the voltage of the incident waves is measured as a function of time by surface electrodes. The surface electrode also relays the voltage from an external voltage source.

Backward directed ultrasound energy would be absorbed by damping layer. This backing material is located behind the piezoelectric element to prevent excessive vibration as well. The reduction of excessive vibration will result in a shorter ultrasonic pulse length and, therefore, improves axial resolution in images (Figure 7). 


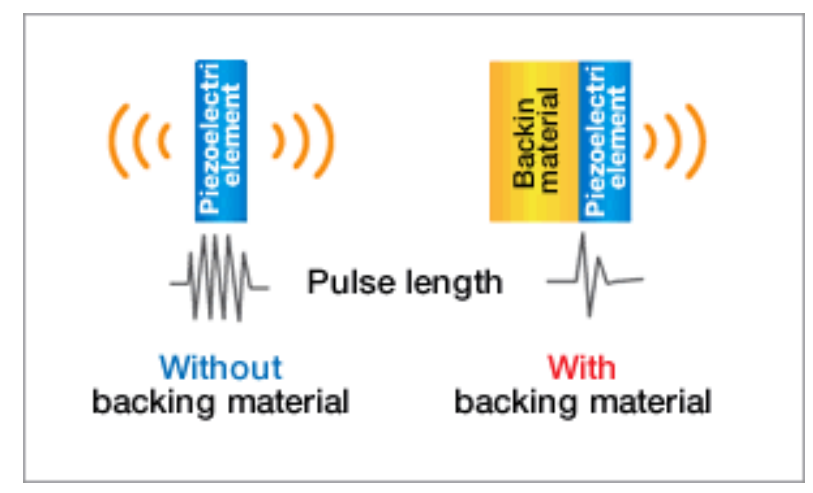

FIGURE 7: DAMPING LAYER. THIS FIGURE SHOWS HOW BACKING MATERIALS WORK [55].

When the piezoelectric element is active, the transmitted ultrasonic waves may be reflected off a target as there is a big difference in acoustic impedance between the piezoelectric element and the object. In order to maximize energy out of the transducer, an impedance matching layer is placed between the object and the face of the transducer. This helps the ultrasonic waves to efficiently penetrate the object. Optimal impedance matching would have the thickness of $1 / 4$ of the desired wavelength. This keeps the reflected waves within the matching layer to be in phase.

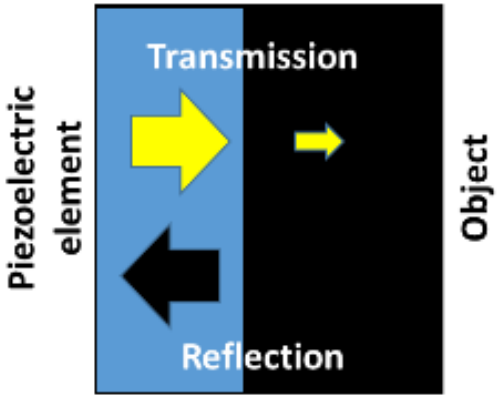

Without an impedance matching layer

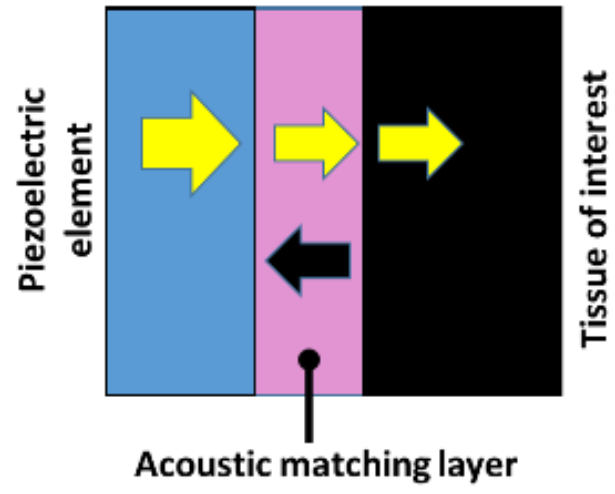

With an impedance matching layer

FIGURE 8: ACOUSTIC MATCHING LAYER. AN INTERMEDIATE MATERIAL IS INSERTED BETWEEN THE TRANSDUCER SURFACE AND THE OBJECT SO THAT ULTRASONIC WAVES CAN EFFICIENTLY ENTER THE OBJECT. TAKEN FROM [55]. 


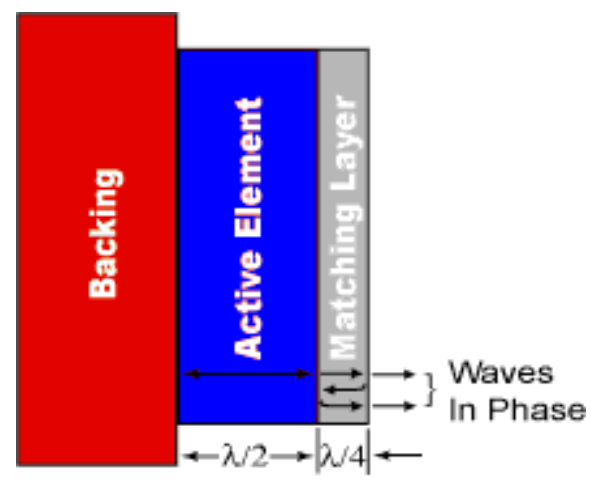

Figure 9: OPtIMAL SIZE OF THE MATCHING LAYER. OPTIMAL IMPEDANCE MATCHING SHOULD HAVE THE THICKNESS OF $1 / 4$ OF THE DESIRED WAVELENGTH. THEREFORE, REFLECTED WAVES WITHIN THE MATCHING LAYER ARE IN PHASE WHEN THEY EXIT THE MATCHING LAYER. TAKEN FROM [56].

Unwanted transmitted vibrations from the piezoelectric element to the transducer case is blocked with the acoustic insulator. The acoustic insulator prevents vibrations originating from the piezoelectric element from being transmitted to the transducer housing while also absorbing external noise. Finally, the housing or case, usually made of hard plastic or metal, protects the internal components of the transducer.

There are typically three types of the ultrasound transducers, which are differentiated based on their capability of coupling to the test media: 1- air-coupled transducers: they use air as the coupling medium between the transducer and test sample. They are especially useful for inspecting sensitive materials which may be negatively affected by liquid coupling agents such as water, gel or oil or physical contact with the transducer. 2- Immersion transducers: they are single element longitudinal wave transducers with a 1/4 wavelength matching layer acoustically matched to water. 3Contact transducers: they are designed to make direct contact with the test material and usually use a water-based gel or highly viscous fluid such as honey or glycerin as a 
coupling medium to reduce the impedance mismatch. These transducers are used in medical diagnostics, non-destructive testing (NDT), and material characterization.

Another important factor in ultrasound transducer function is the range of the frequency in which they operate. In pulse echo ultrasound imaging, resonance transducers are manufactured to operate in a "resonance" mode with a high voltage of very short duration (commonly $150 \mathrm{~V}$ with spike of $\approx 1 \mathrm{msec}$ ). This high voltage spike causes contraction of the piezoelectric material and, therefore, the vibration at a natural resonance frequency. The thickness of the material determines the resonance frequency in which the ultrasound transducer should operate. Such a thickness should be half of the wavelength of ultrasound waves to be transmitted. The operating frequency is, therefore, calculated based on the speed of the sound in the piezoelectric materials as well as the thickness $(0.5 \lambda)$ of them [57].

Resonance transducers transmit and receive preferentially at a single resonance frequency. Higher frequencies could be achieved with thinner elements, and lower frequencies with thicker elements [57]. 

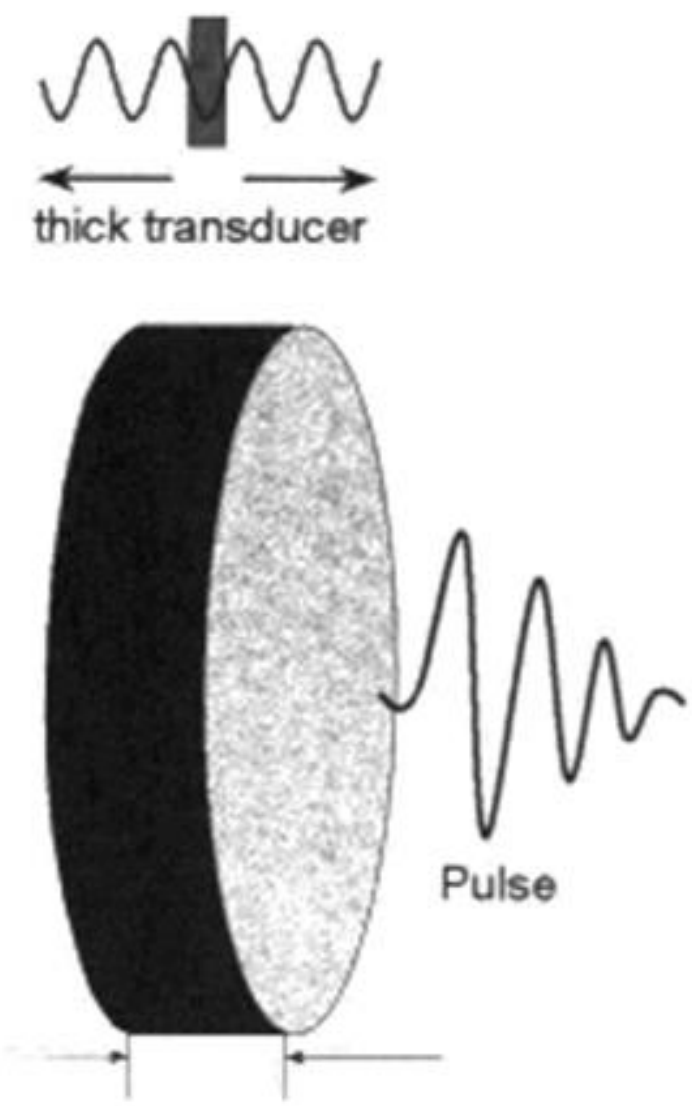

Low frequency
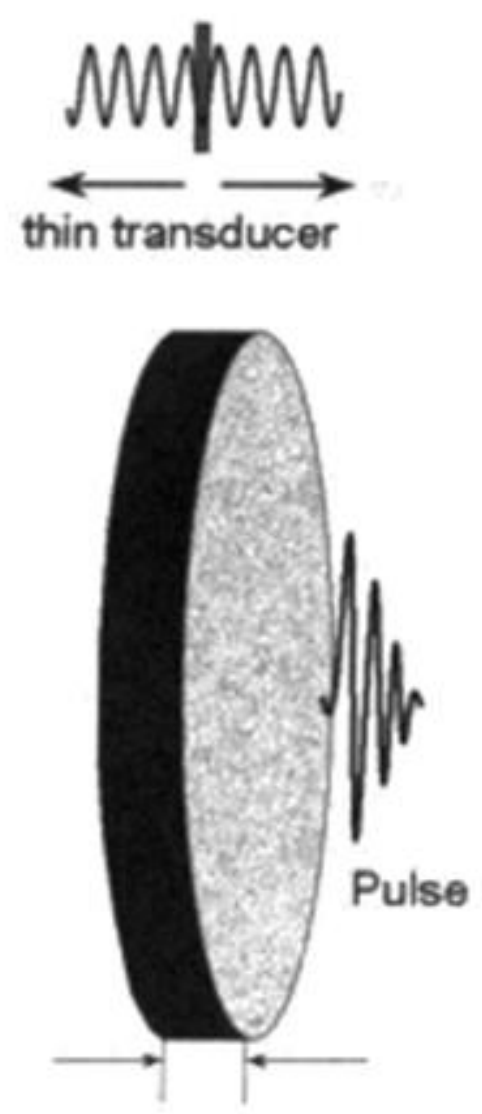

High frequency

FIGURE 10: TRANSDUCER THICKNESS. RESONANCE FREQUENCY IS DETERMINED BY THE TRANSDUCER THICKNESS EQUAL TO 0.5 $\wedge$. TAKEN FROM [57].

The addition of a damping block gives the ultrasound pulse width a short spatial pulse length. Such a damping of vibration (known as ring-down) decreases the purity of the resonance frequency and, therefore, make a broadband frequency spectrum. In other words, ring-down causes an increase in the range of frequencies in the ultrasound pulses to have higher and lower frequencies above and below the resonance frequency. The applications of a broad bandwidth transducers are mostly in imaging where high spatial resolution along the direction of the beam is required. However, in Doppler ultrasound, 
narrow-band transducers are used, as the velocity information encoded by changes in the echo frequency relative to the incident frequency should be preserved.

\subsection{Ultrasonic Transducer Array}

The majority of ultrasound systems consist of transducers with many individual rectangular piezoelectric elements forming linear or curvilinear arrays (Figure 11). Most of the arrays have 128 to 512 individual rectangular elements. The width of each element is typically less than half the wavelength and a length of several millimeters.

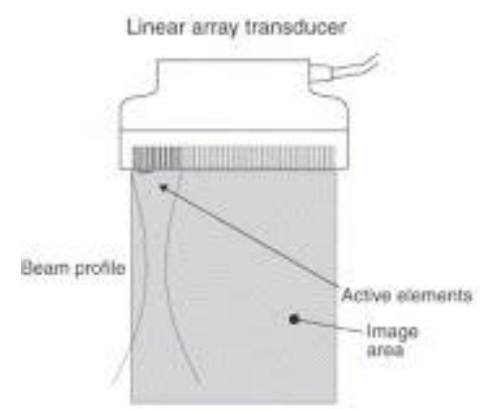

Phased array transducer

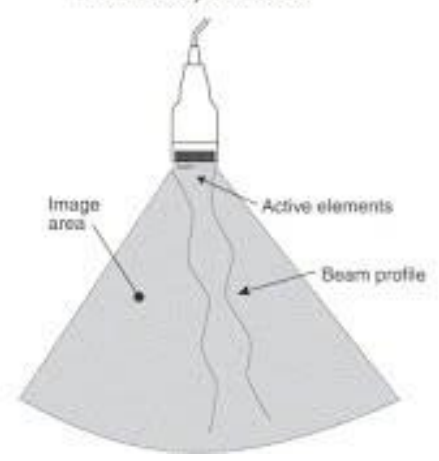

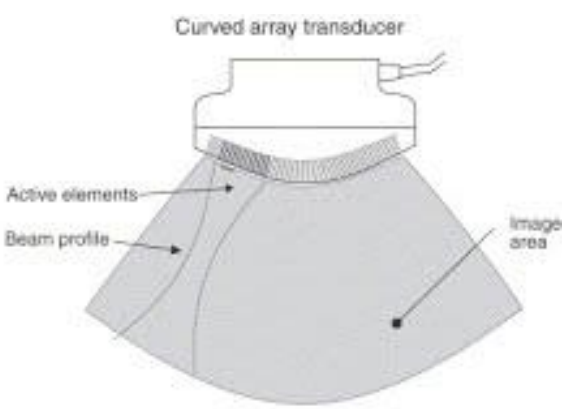

Sector scan transducer

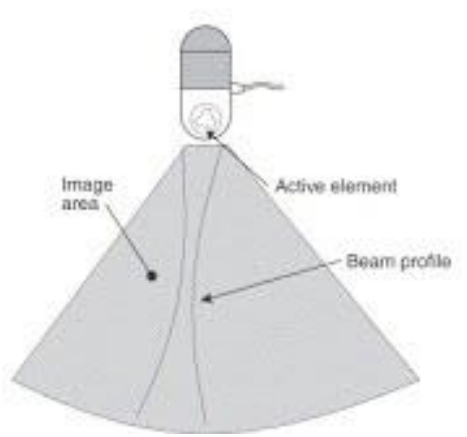

Figure 11: TRANSDUCER ARRAYS. DifFERENT TYPES OF TRANSDUCERS. TAKEN FROM [58].

A beam is produced from two different modes of activation: linear (sequential) and phased activation/ receive modes. In linear arrays, the ultrasound beam is formed by the simultaneous firing of a small group of adjacent elements $(\approx 20)$. Then, echoes would be collected from most of the transducer elements. This would be continued by firing 
another group of transducer elements displaced by one or two elements and then collecting received echoes by most of the elements. In linear array, a rectangular or a trapezoidal field of view can be produced by linear or curved linear transducer arrays (Figure 11).

In a phased array, however, the discrete elements of transducers will be activated by applying a time delay. This causes the ultrasound beam to be steered and focused electronically without moving the transducer. Then, all of the elements collect the received echoes. These received echoes will then be used for image reconstruction with more sophisticated algorithms.

\subsection{Ultrasound Pressure Waves}

Ultrasound energy requires a medium to propagate. Ultrasound is the result of propagation of the mechanical energy through the medium. Sound waves would be transmitted from the mechanical vibrations of a source of ultrasound to propagating medium which the source is in contact with. Such sound waves are transmitted as a series of pressure waves with high and low pressure pulses (Figure 12).

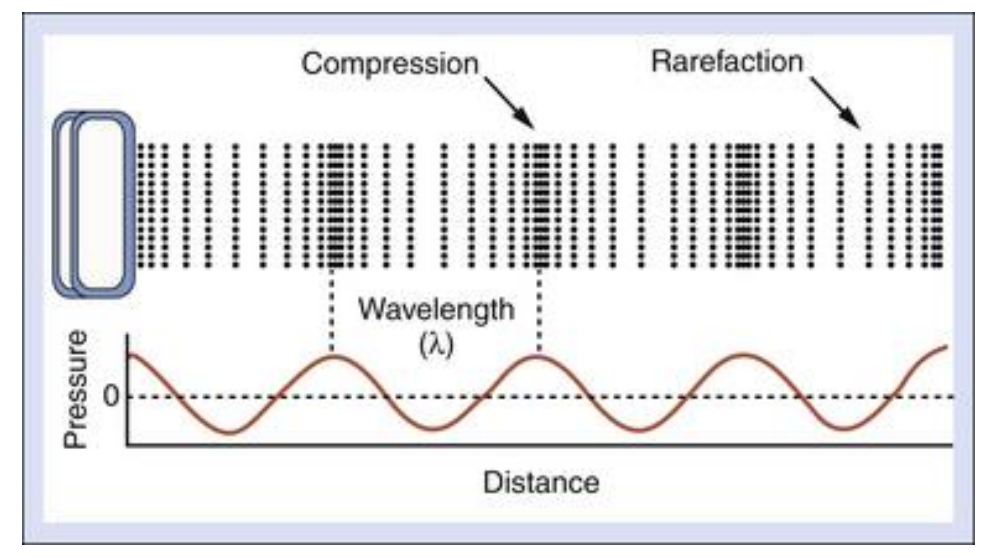

Figure 12: Propagation of Ultrasound through a MEDIUM. THIS FigURE SHOWS the VARIATION OF PRESSURE IN THE PROPAGATION OF ULTRASOUND. TAKEN FROM [59]. 
In Figure 9, compression at high pressure areas would be happened when the particles in the medium squeezed together and conversely, rarefaction at low pressure areas would be happened when the particles spread apart. The distance in which the particle is displaced is considered as the amplitude of the ultrasound.

The propagation direction of the particles' displacement in the medium defines the propagation mode of the sound wave. There are four principle modes of propagations based on the particles oscillation in solid. Sound can propagate as longitudinal waves, shear waves, surface waves, and plate waves (in thin materials). In ultrasound testing, Longitudinal and shear waves are the two widely used of the modes of propagation (Figure 13) [60].

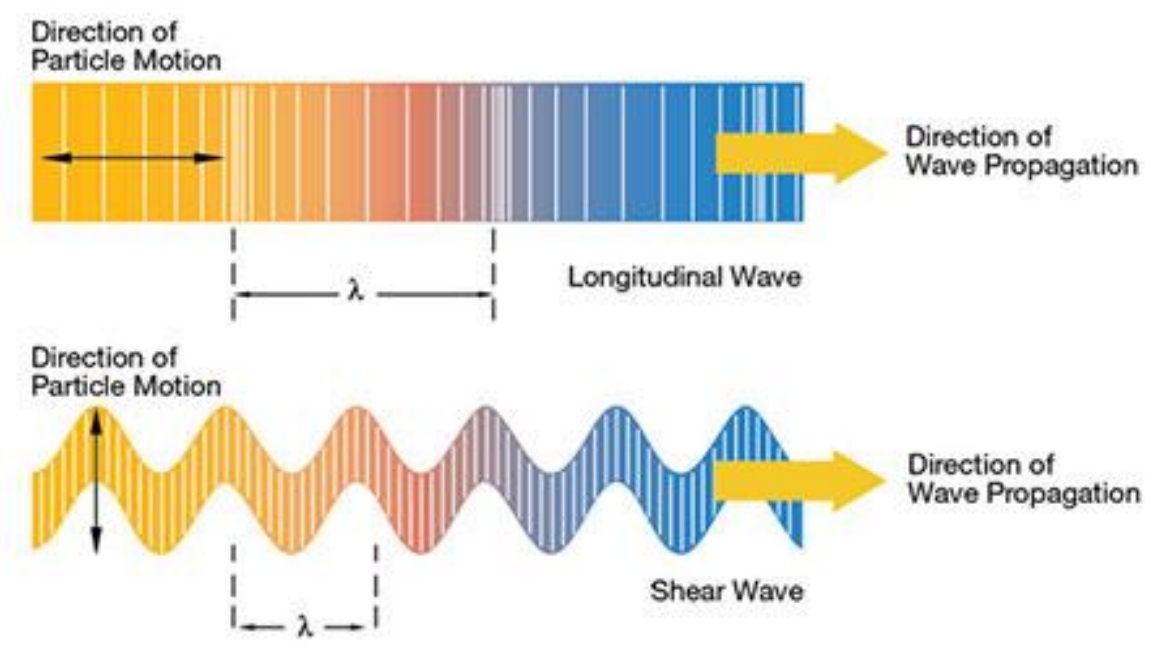

FigURE 13: WAVE PROPAGATION. LONGITUDINAL WAVES VS SHEAR WAVES. TAKEN From [61].

In a longitudinal wave, the direction of the particle motion in the medium is parallel to the direction of the wave front. Audible sound waves propagate in longitudinal mode. In contrast, when the direction of the particle motion is perpendicular to the direction of the wave, it would be considered as shear waves. Shear wave has shorter 
wavelength and smaller velocity than longitudinal wave. When the oscillating motion travels along the surface of a test piece with the depth of one wavelength, it refers to the surface waves, also known as Rayleigh waves.

\subsubsection{Ultrasound Wave Properties}

The propagation of the ultrasound wave through a medium causes periodic movements of the particles. Such particles periodic movements result in fluctuations in pressure. These fluctuations could be represented as a sinusoidal curve (Figure 12). Such a sinusoidal curve is characterized by the following parameters.

The magnitude of particle displacement from its mean position gives us the amplitude for that specific moment. When this amplitude reaches the maximum particle displacement, it is known as peak amplitude which represents the maximum change in pressure. If the amplitude against distance is ploted, the distance between every two corresponding positions on the sine wave is known as wavelength $(\lambda)$. Therefore, in one complete wave cycle, wavelength represents the distance in which the pressure wave is propagated and it shows one vibration of the source transducer.

The number of times the wave is repeated per unit of time is known as frequency

(f). Reciprocally, the time for one complete cycle is known as period $\left(T=\frac{1}{\mathrm{f}}\right)$. So the total propagated distance by the wave in unit of time can be achieved by the product of wavelength and frequency. This is known as velocity of the wave $(c=\lambda \times f)$.

Attenuation of the ultrasound in biological tissues are highly related to the frequency. When the frequency is higher, the energy of the wave would be attenuated 
faster through a medium. Therefore, depth of penetration would be higher in lower frequencies. Table 1shows Frequencies used in some common diagnostic applications.

TABLE I: IMPORTANCE OF TRANSDUCER FREQUENCY IN CLINICAL ULTRASOUND APPLICATIONS. NOTE THAT LOWER FREQUENCIES ARE USED TO REACH FARTHER WITHIN THE BODY, SUCH AS THE ABDOMEN. TAKEN FROM [52].

\begin{tabular}{|c|l|}
\hline Frequency $(\mathrm{MHz})$ & \multicolumn{1}{c|}{ Application } \\
\hline 2.5 & Deep Abdomen. OB/Gyn \\
3.5 & General Abdomen. OB/Gyn \\
5.0 & Vascular, Breast, Gyn, \\
7.5 & Breast, Thyroid \\
10.0 & $\begin{array}{l}\text { Breast, Thyroid, Superficial Veins, } \\
\text { Superficial Masses } \\
\end{array}$ \\
\hline
\end{tabular}

The ability to distinguish two adjacent objects in space is called spatial resolution. When the distinguishable distance between two objects is smaller, the spatial resolution is higher. The spatial resolution is known as axial and lateral resolution. Frequency and wavelength are two major factors in determining the spatial resolution.

\subsubsection{Beam Properties}

The ultrasound beam leaves the transducer surface in the form of a wave in the longitudinal direction. The waves are typically 2 or 3 cycles (wavelengths) of the same frequency. The length and the width of the pulse determines the axial and the lateral resolutions, respectively (Figure 15) [52]. Axial resolution (also known as longitudinal, depth or linear resolution in the direction parallel to the ultrasound beam) is independent of the depth in which the pulse is propagated and is the same at any point along the beam. Axial resolution can be defined as follows: 
- Axial resolution $=$ spatial pulse length/2 or $(\#$ cycles in the pulse $\mathrm{x}$ wavelength $) / 2$ Therefore, axial resolution can be improved by shortening the length of the ultrasound pulse. This could be done by decreasing the number of cycles in the pulse or increasing the frequency of the pulse. Therefore, with a higher frequency comes shorter pulse length, and, therefore, higher axial resolution. However, by increasing the frequency, we would have less depth of penetration.
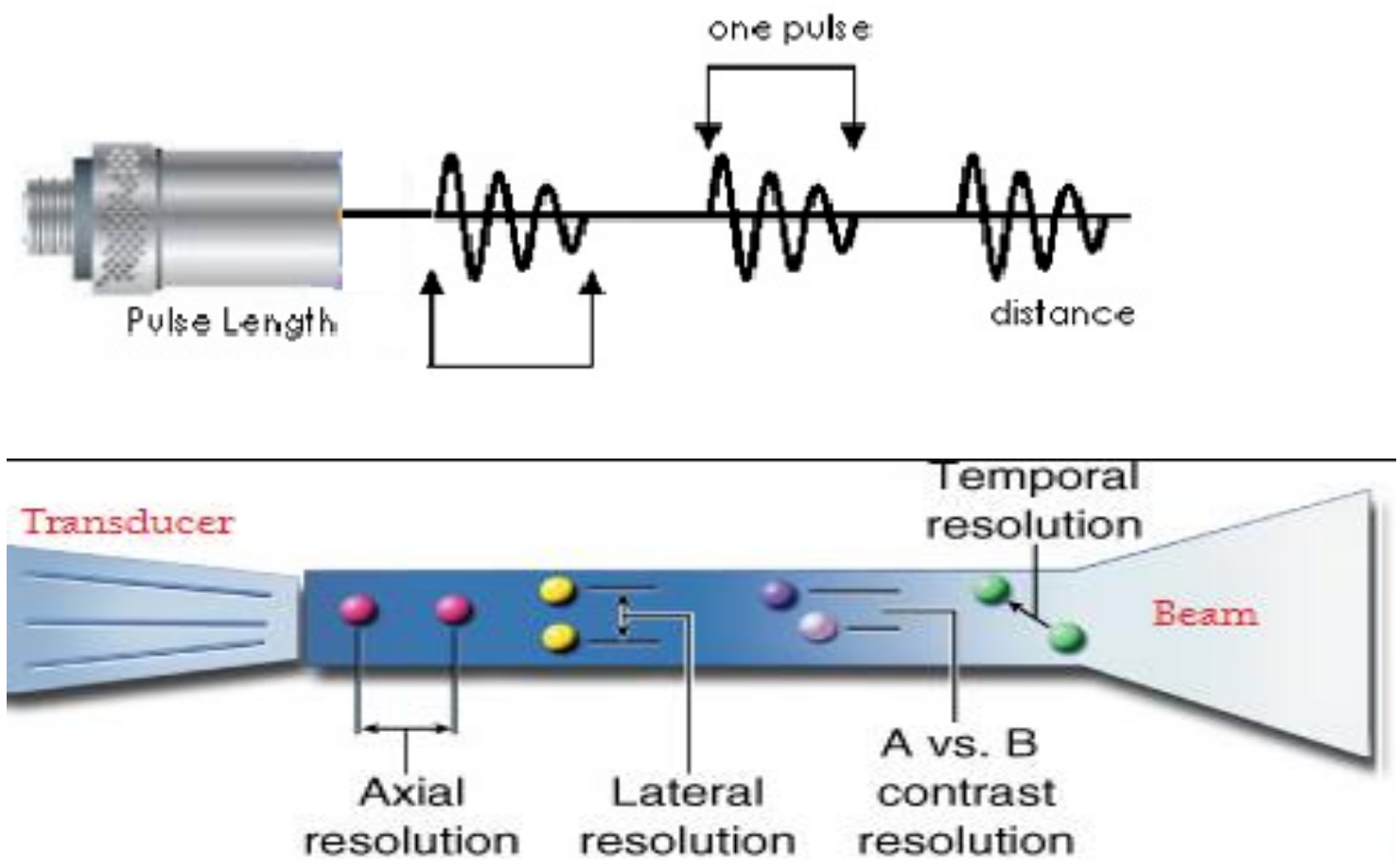

FIGURE 14: AXIAL AND LATERAL RESOLUTION. THE LENGTH AND THE WIDTH OF THE PULSE DETERMINES THE AXIAL AND THE LATERAL RESOLUTIONS, RESPECTIVELY. AXIAL RESOLUTION IS IN THE PARALLEL DIRECTION TO THE ULTRASOUND BEAM; EQUALS TO ONE-HALF THE SPATIAL PULSE LENGTH. LATERAL RESOLUTION IS ABILITY TO DISTINGUISH TWO OBJECTS IN PERPENDICULAR DIRECTION TO THE DIRECTION OF THE ULTRASOUND BEAM. TAKEN FROM [SHERONICA] AND [62].

In children and superficial structures, in which penetration is not important, higher frequency ultrasound provides better resolution. In contrast, for deep structures, 
where deeper penetration is required, lower frequency transducers are used but at a tradeoff of lower resolution [52].

The ability to distinguish two points in the direction perpendicular to the direction of the ultrasound beam propagation is called lateral resolution. Lateral resolution is related to the width of the beam and the depth of imaging. Lateral resolution would be decreased by wider beams as they typically diverge further. In addition, any ultrasound beam diverges at greater depth, will result in lower lateral resolution. Therefore, lateral resolution is worse with deeper depths and best at shallow depths.

When two points lying side by side are located within the same beam width, Lateral resolution is poor as the returning echoes overlap with each other and, therefore, the resulting image displays the points as one. In practice, it is very important to keep the beam width as narrow as possible by choosing the highest frequency transducer to maximize lateral resolution. However, the trade of choosing either higher frequency or more penetration should be taken into account.

The ability to distinguish between objects with different intensity in an image refers to contrast resolution (Figure 15), and temporal resolution is the ability to detect object movement over time by ultrasound. Temporal resolution depends on the frame rate:

- $\quad$ The temporal resolution or frame rate $=1 /($ time to scan one frame $)$.

- The time to scan one frame $=$ pulse repetition period $\times$ number of scan lines per frame. 
In order to improve the temporal frequency, we can decrease the pulse repetition period by decreasing the depth of imaging or narrowing the imaging sector, which decreases the time it takes to scan one frame.

The ultrasound beam has two distinct patterns, called near field and far field (Figure 16). In near field (also known as Fresnel zone), the beam is slightly converging out to a distance specified by the geometry of the transducer and the frequency. It is adjacent to the transducer face. Multiple constructive and destructive interference patterns of the ultrasound waves from the transducer surface makes the beam converge in the near field. The near field length can be calculated by equation (1).

$$
\text { Near field length }=\frac{d^{2}}{4 \lambda}=\frac{r^{2}}{\lambda}
$$

$r$ is the transducer radius, $d$ is the transducer diameter, and $\lambda$ is the wavelength of ultrasound in the propagation medium. Lateral resolution peaks at the end of the near field for a transducer with single element and decreases as it is moved away from the transducer surface.

In far field (also known as Fraunhofer zone), the beam is diverging, starting at the end of the near field. The angle of the beam divergence in the far field can be calculated by equation (2):

$$
\operatorname{Sin}(\theta)=1.22 \frac{\lambda}{d}
$$

$\lambda$ is the wavelength and $\mathrm{d}$ is the effective diameter of the transducer. Therefore, by using large diameter transducers and high frequency, beam divergence would decrease. 
In a transducer array, several elements operate at the same time to form the ultrasound beam. The width of each element is typically less than one wavelength to produce a diverging beam at close distance to the elements surface. When a group of selected elements in an array simultaneously fire an ultrasound beam, a transducer width equal to the some of the individual elements would be formed and the final beam, compiled from the individual beams, would have the same properties of a single transducer of the same size of the active elements. The focal distance in the linear array is a function of the transducer diameter or the width of the group of simultaneously activated elements, resonance frequency, and the presence of any acoustic lenses attached to the surface of the elements. 


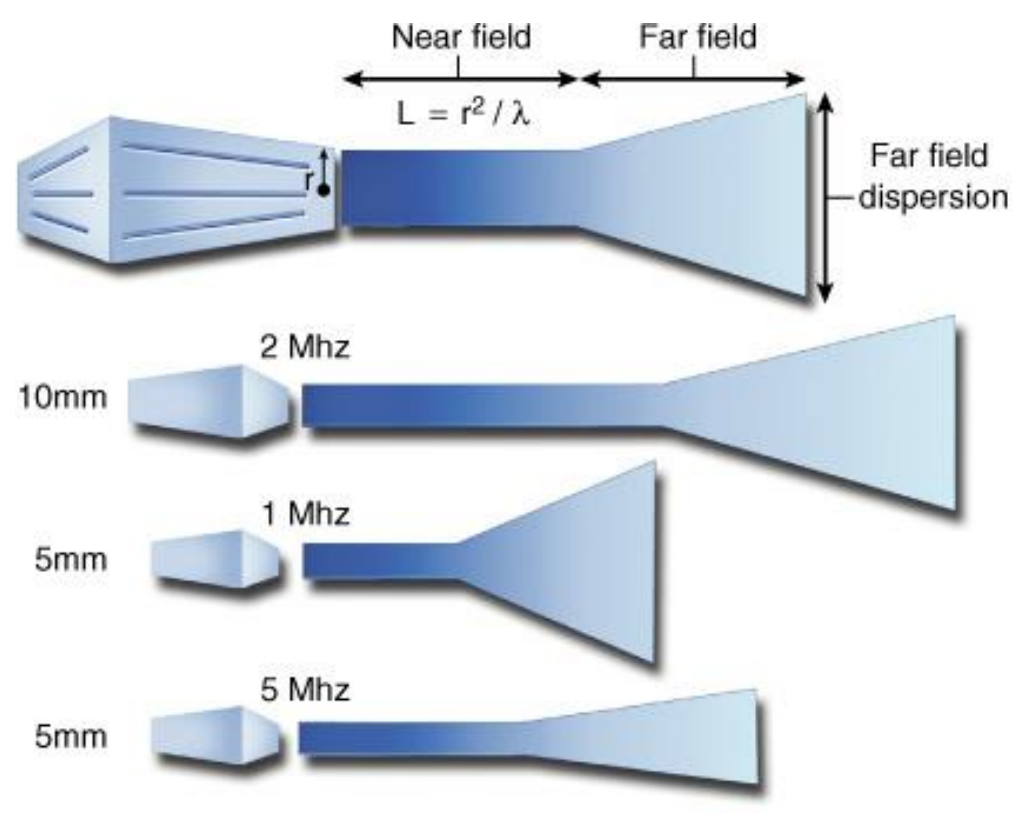

FIGURE 15: SCHEMATIC OF A TYPICAL ULTRASOUND TRANSDUCER. THE ULTRASOUND TRANSDUCER EMITS ULTRASOUND FROM A SERIES OF PIEZOELECTRIC CRYSTALS. THE BEAM HAS SEVERAL COMPONENTS, INCLUDING A NEAR FIELD (FRESNEL ZONE) WITH RELATIVELY NARROW BEAM WIDTH AND A FAR FIELD WHERE THERE IS DIVERGENCE OF THE ULTRASOUND BEAM. ULTRASOUND INTENSITY DECREASES WITH DISTANCE FROM THE

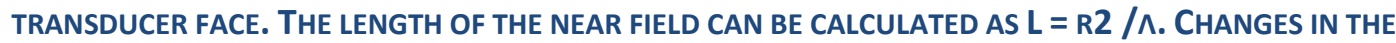
TRANSDUCER DIAMETER AND FREQUENCY HAVE PREDICTABLE EFFECTS ON THE NEAR AND FAR FIELD CHARACTERISTICS, AS IS NOTED IN THE LOWER TWO ILLUSTRATIONS. HIGHER FREQUENCIES PRESERVE THE LENGTH OF THE NEAR FIELD COMPARED TO LOWER FREQUENCIES, AS DO LARGER TRANSDUCER DIAMETERS. TAKEN FROM [62].

In a phased-array transducer, elements are activated with a slight difference in excitation time. The beam is produced with the interaction of the individual wave fronts from each element. Such phase differences in the elements can steer of focus the beam profile. The focal distance in phased-array transducers and many linear array transducers is adjustable and can be adjusted by applying timing delays to the activated elements (Figure 17). 

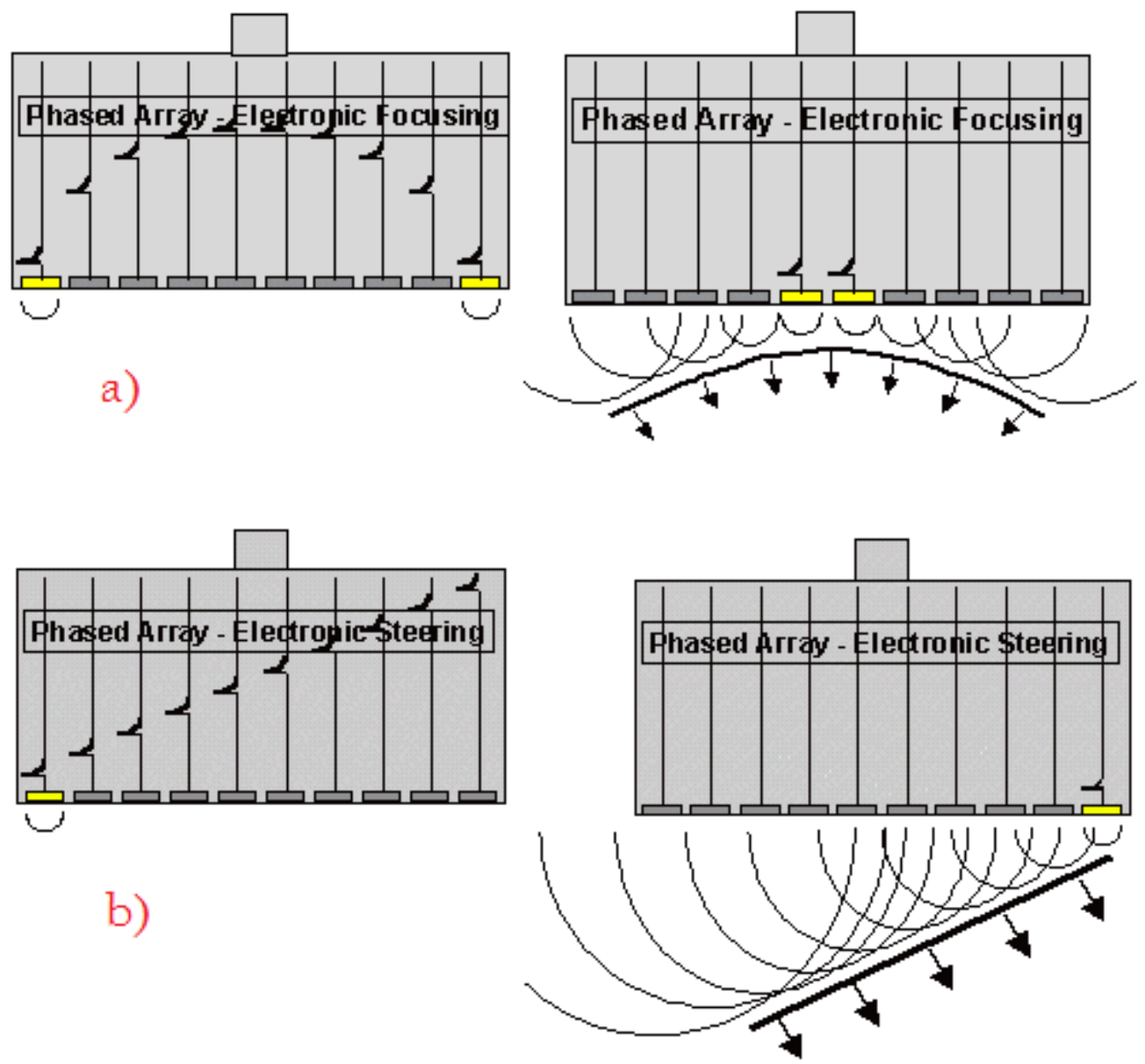

FIGURE 16: PHASED ARRAY. BY APPLYING A TIME DELAY TO FIRE ULTRASOUND SIGNALS FROM DIFFERENT ELEMENTS, WE CAN CONTROL EITHER THE DIRECTION OF PROPAGATION OR THE DEPTH OF THE FOCAL ZONE. TAKEN FROM [63].

In phased-array transducers, for producing a shallow focal zone, for example, the outer elements should fire the wave before the activation of the inner elements. By reducing the delay time in firing the waves from outer elements to inner elements, we can produce greater focal distances. It is also possible to create multiple focal zones by repeatedly firing and collecting data with the same number of elements but with different delays in sending the waves from the elements (Figure 18). 
The received echoes would be collected by all the elements and are summed to create the ultrasound signal. As the received echoes at the edge are travelling a longer distance (compared to ones collected at the center of the array), signals from each individual elements should be rephrased to avoid loss of resolution.

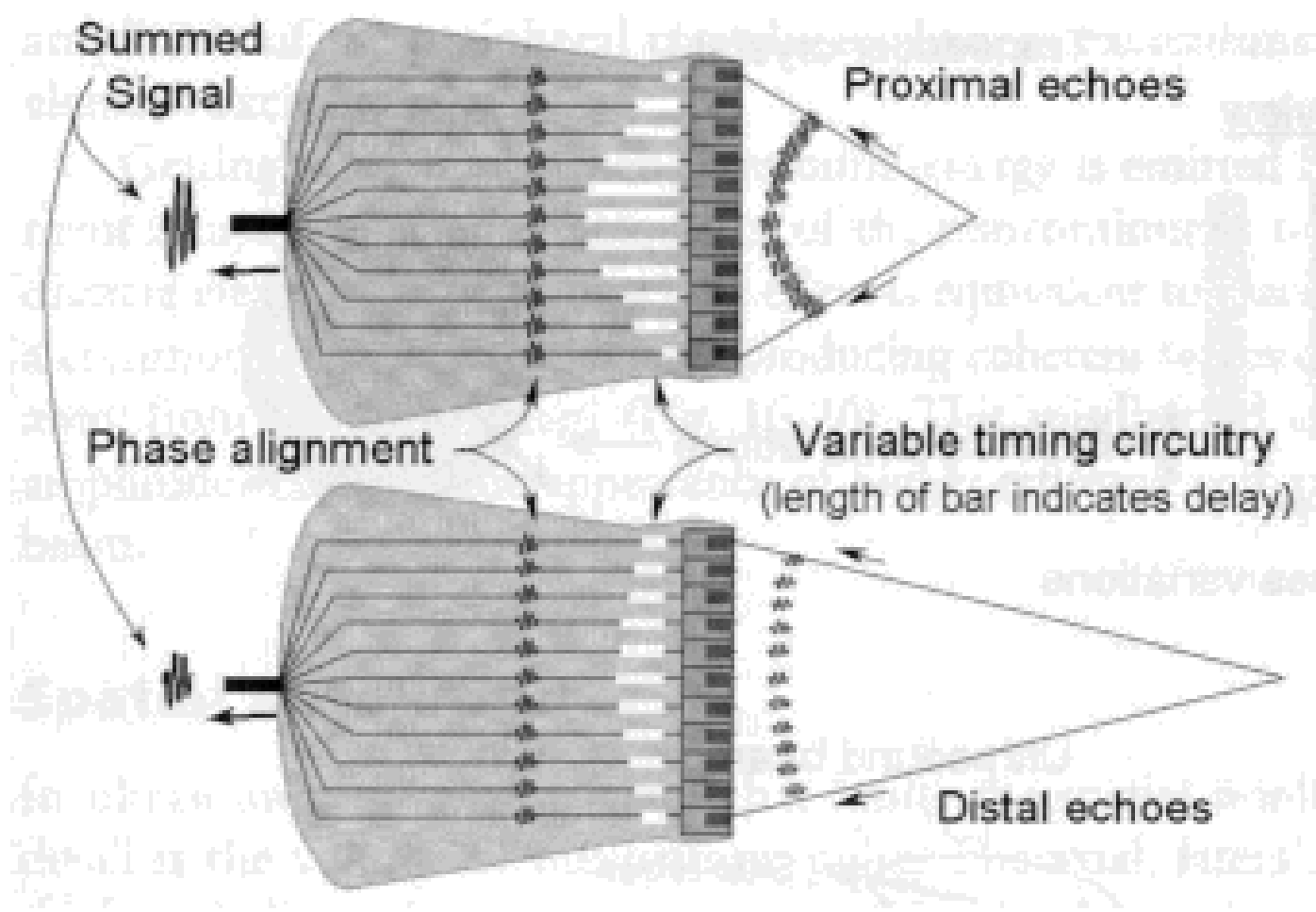

FiguRE 17: ADJUSTABLE FOCAL ZONE IN PHASED-ARRAY TRANSDUCERS. TAKEN FROM [57].

\subsection{Interaction of the Ultrasound with Body Tissues}

Ultrasound can have different responses depending on the acoustic property of different tissues. This property of tissue is called acoustic impedance $(z)$. It describes the amount of resistance an ultrasound encounters as it passes through a tissue. Acoustic impedance 
depends on two factors (equation (3)): the density of the tissue $(\rho$, in $\mathrm{kg} / \mathrm{m} 3)$ and the speed of the sound wave $(\mathrm{c}$, in $\mathrm{m} / \mathrm{s})$.

$z=\rho \times c$

Therefore, if the density of the tissue or the speed of the sound increases, the acoustic impedance would also be increased. For example, the lowest acoustic impedance is for air-containing organs such as the lung, and the highest acoustic impedance is for body parts with the highest density, like bone. When ultrasound propagates through the body, it interacts with tissue in four manners. It could be reflected back at the interface of two body tissues towards the transducer as the tissues have different acoustic impedances. Reflection could also happen if the tissue boundary width is bigger than the wavelength of the ultrasound wave. In addition, if there is any angle less than 90 degrees between the ultrasound beam and the tissue boundary, the ultrasound beam has a chance to be reflected.

If the ultrasound signal is deflected from a straight path, it would refer to refraction. This happens at a different medium interface with different acoustic impedance and can cause artifacts. If the width of the tissue boundary is less than one wavelength of the ultrasound beam, scattering will happen. The reflected signal that reaches the transducer is much weaker compared to the transmitted signal. Scattering mostly occurs at interfaces involving structures of small dimensions, like red blood cells with average diameter of $7 \mu \mathrm{m}$. Diffuse reflection also can be the result of interaction of the incident pulse with a rough, irregular tissue interface. Figure 19 shows different interactions of ultrasound with tissue boundaries. 


\section{Reflection}
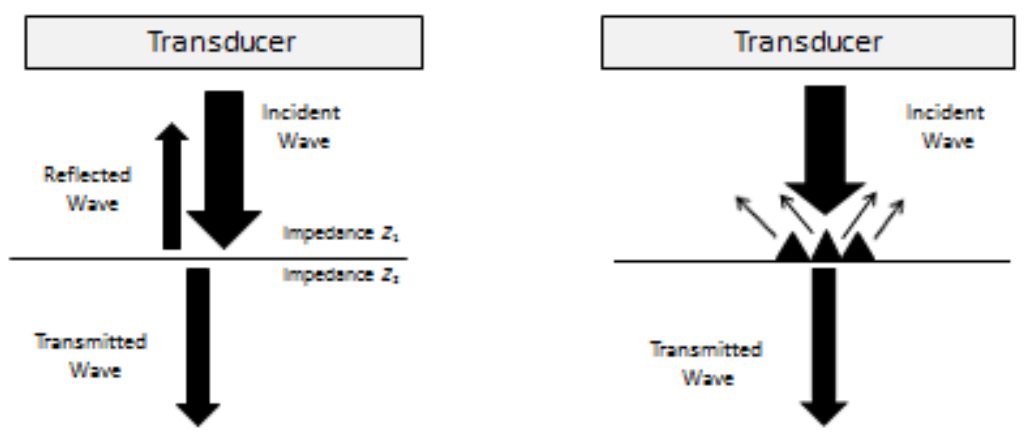

\section{Refraction}
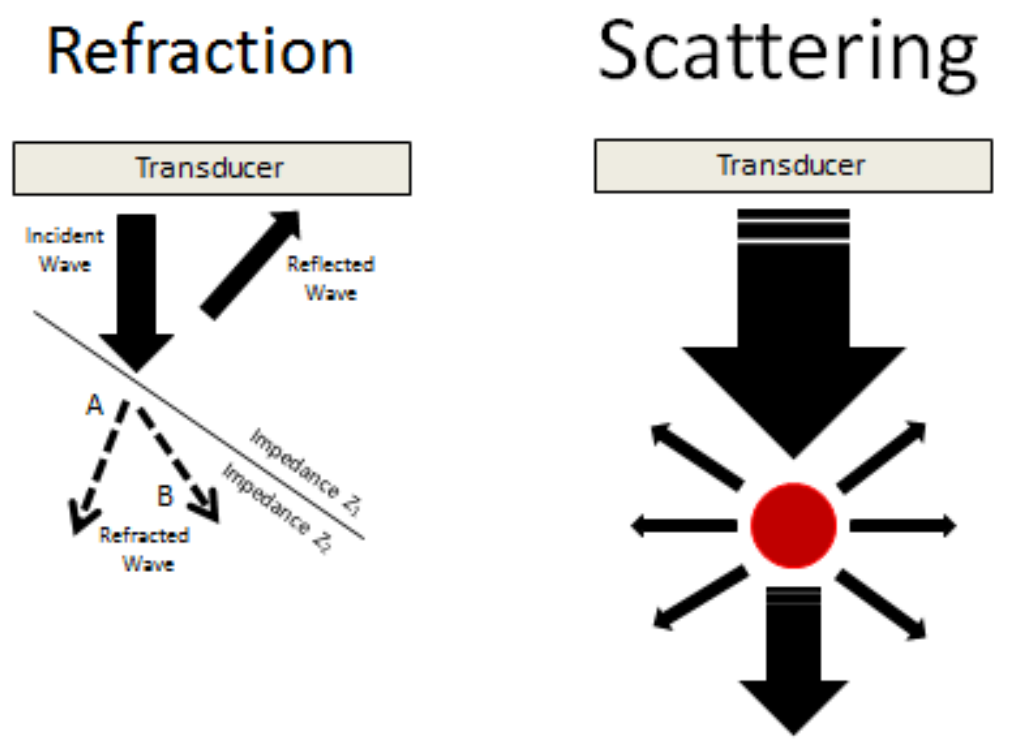

FIGURE 18: ULTRASOUND INTERACTION WITH TISSUE. TAKEN FROM [64].

When the ultrasound wave propagates through a medium, its intensity decreases as a function of distance. This is known as attenuation. In attenuation, the medium absorbs some of the energy of the ultrasound wave. Therefore, as the ultrasound travels through the medium, it loses its energy by reflection, scattering, and attenuation.

Attenuation is also dependent on the frequency. In order to evaluate the attenuation of the ultrasound intensity as a function of frequency, attenuation coefficients 
$(\alpha)$ are used. Attenuation coefficient varies for different media. It can be used to calculate the total attenuation in the medium (equation (4)).

$$
\text { Attenuation }=\alpha \times l \times f
$$

$\alpha$ is attenuation coefficients, $l$ is the medium length, and $f$ is the frequency. The unit of the total calculated attenuation is in $\mathrm{dB}$. Figure 19 shows the attenuation for various body tissues as a function of the frequency used in ultrasound.

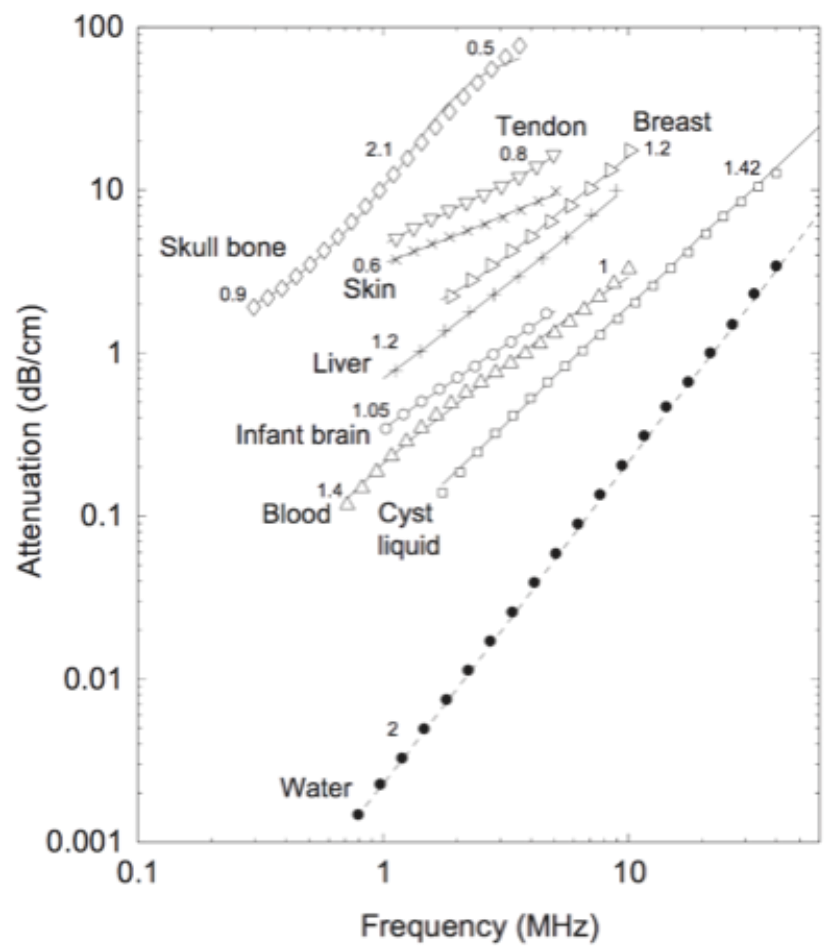

FigURE 19: ATTENUATION FOR VARIOUS BODY TISSUES AS A FUNCTION OF THE FREQUENCY. TAKEN FROM [65].

From Figure 19, it is shown that the skull bone has the highest attenuation compared to other body tissues. One of the biggest challenges of designing a noninvasive ultrasonic based imaging system for the brain is the amount of energy loss caused by the human skull. Skull bone attenuates the ultrasound wave energy about $90 \%$ even with low frequency ultrasound $(<1 \mathrm{MHz})$ is being used. 
The major causes of attenuations are due to reflection, scattering, and mode conversion [52]. The skull bone consists of a layer of cancellous (spongy) bone called diploë. It is located inside the layers of compact bone (Figure 20).

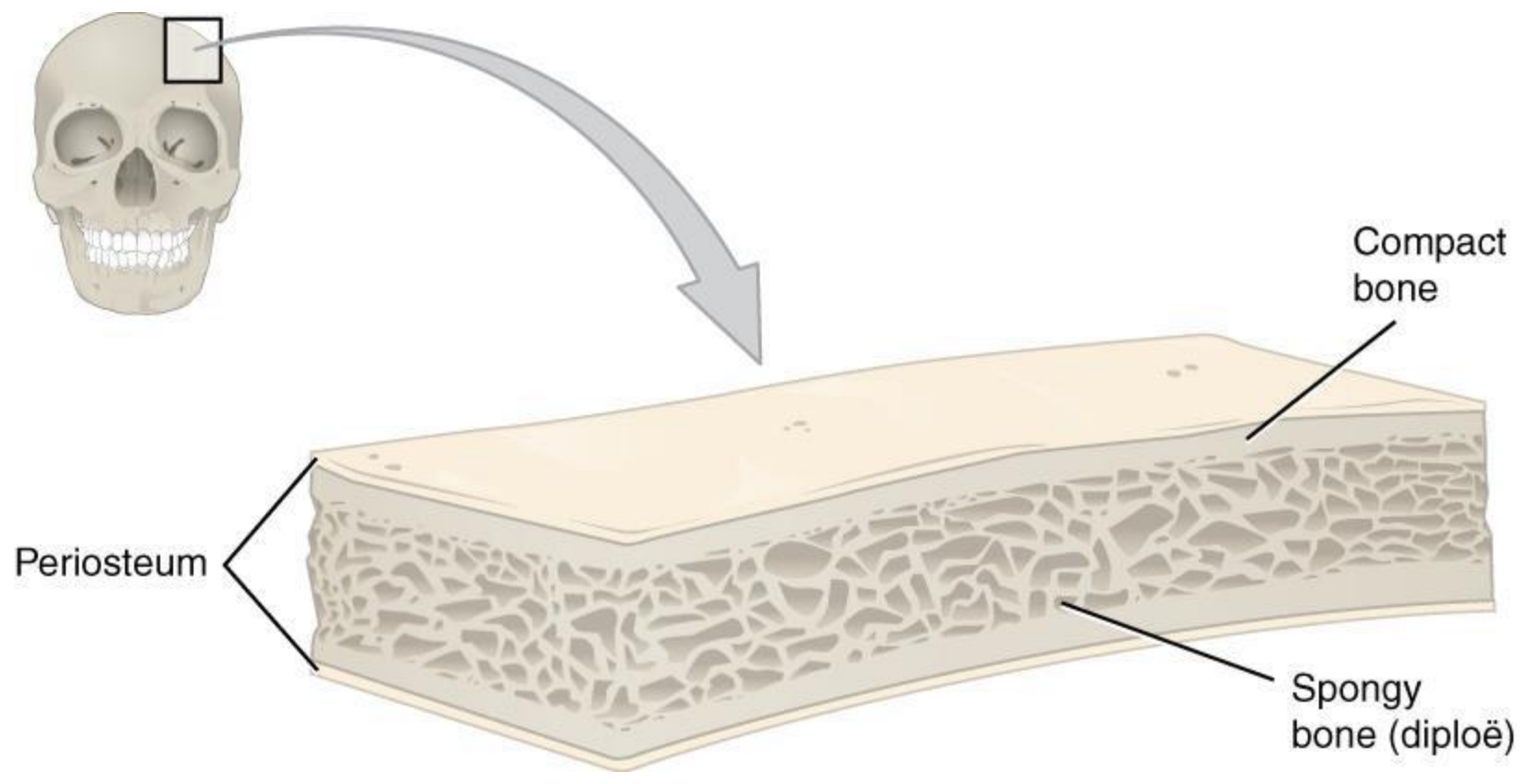

FIGURE 20: HUMAN SKULL STRUCTURE. TAKEN FROM [52].

The two layers of compact bone in human skull are denser than spongy bone. The spongy bone is filled with fluid-like marrow. In addition to the porosity structure of the diploë, differences in mechanical properties between the marrow and the bone highly scatter the ultrasound [52]. Therefore, the conventional pulse-echo method for imaging the brain is not practical as the intensity of the reflected signal from soft tissue is normally too weak to penetrate the highly attenuating skull bone and cannot be detected by the transducers. Therefore, noninvasive imaging of the brain requires a system which is capable of sending maximum energy inside the skull with minimum reflection and then detecting weak scattered signals outside the human skull. 


\section{CHAPTER III}

\section{BACKGROUND}

Following is a brief overview of the application of ultrasound applied noninvasively in the human brain. Due to the limitations of applying high frequency ultrasound energy through the human skull, there is no known ultrasonic imaging systems capable of taking images of the human brain. However, current applications of ultrasonic systems for focusing energy inside the brain (Section 3.1), different types of transducer arrays (Section 3.2), and Ultrasound tomography image reconstruction algorithms (Section 3.3) are briefly reviewed.

\subsection{Transcranial Ultrasound}

The purpose of this section is to introduce existing biomedical applications of the ultrasound in human brain. Over the past 20 years techniques for focusing ultrasound through the human skull have been developed for therapeutic treatment of the brain. In the current work, the problem is more difficult: energy must not only be directed through 
the skull, but there is also the need to collect the scattered signal for image reconstruction.

One of the non-invasive applications of focused ultrasound utilizes its capability to thermally ablate tissue. It is possible to focus the ultrasound beam deep into soft tissue. High intensities ultrasound beams are absorbed resulting in localized heating in the focal zone. This procedure can be done without damaging the surrounding tissues. Focus of the beam can be done by guidance from Ultrasound imaging to target the beam [reference] and, more significantly, MRI for targeting and providing feedback control of the procedure with temperature imaging [66]. The combination of such imaging systems with focused ultrasound has brought about several focused ultrasound systems designed for mostly soft tissue targets [67]-[70]. When the tissue heated up more than the threshold for protein denaturation at around 57-60 degrees Celsius, result in the necrosis of the cells depending upon a very predictable temperature-time relationship [71]-[74].

Ultrasound is a pressure wave that propagates through a medium. As the wave travels, pressure variations causes microscopic shearing that subsequently are mechanically converted into heat [75]. This is the main process of the heating in soft tissue during sonication (the ultrasound exposures). Another factor is the non-linear propagation of the ultrasound wave during sonication which can cause excess heating [76]-[79]. The process highly depends on the medium characteristics, wave amplitude, and distance traveled. Therefore, high-intensity focused ultrasound is also a big factor to consider in generating heat [80].

Focusing ultrasound inside the brain has been prevented due to the interaction of the skull on the ultrasound propagation. Compared to soft tissues, bone has around 30-60 
times more acoustic attenuation resulting in rapid heating in the skull and thus limits the exposure levels that can be safely applied [22]. A solution to the problem was addressed in [22] by designing a hemispherical transducer operating at a lower frequency for ultrasound beam generation and actively cooling down the scalp [18], [22], [35]. Overheating can be prevented by designing hemispherical transducers to distribute the resulting bone heating over a large enough area and lower frequency (700 kHz [47]) can be used for generating ultrasound to reduce absorption in the skull. The gain also was increased by designing large geometric aperture to have sufficient focal intensity for ablation even at lower frequencies [22].

One of the studies [22] designed and tested a clinical prototype of a phased array transcranial MRI-guided focused ultrasound surgery (TcMRgFUS) [3], [4], [22], [81]. The prototype is based on extensive preclinical acoustic [18], [35], [41], [82], [83] and MRI [66], [84]-[86] studies.

Technical results of patient treatments as well as the feasibility of the focusing ultrasonic beam through the skull was reported [87]. The increase of the temperature at focal area and the surface of the brain was evaluated using MRI [22], [88]. Figure 21 shows the procedure that how the newly developed focused ultrasound device would use high intensity beams to ablate the tissue non-invasively, INSIGHTEC LTD, MRgFUS, Carmel-Tirat, Israel. This device is approved by food and drug administration (FDA) to treat patients with essential tremor [89]. As the procedure is non-invasive, there is no need of the ionizing radiation, incisions, or implants. 

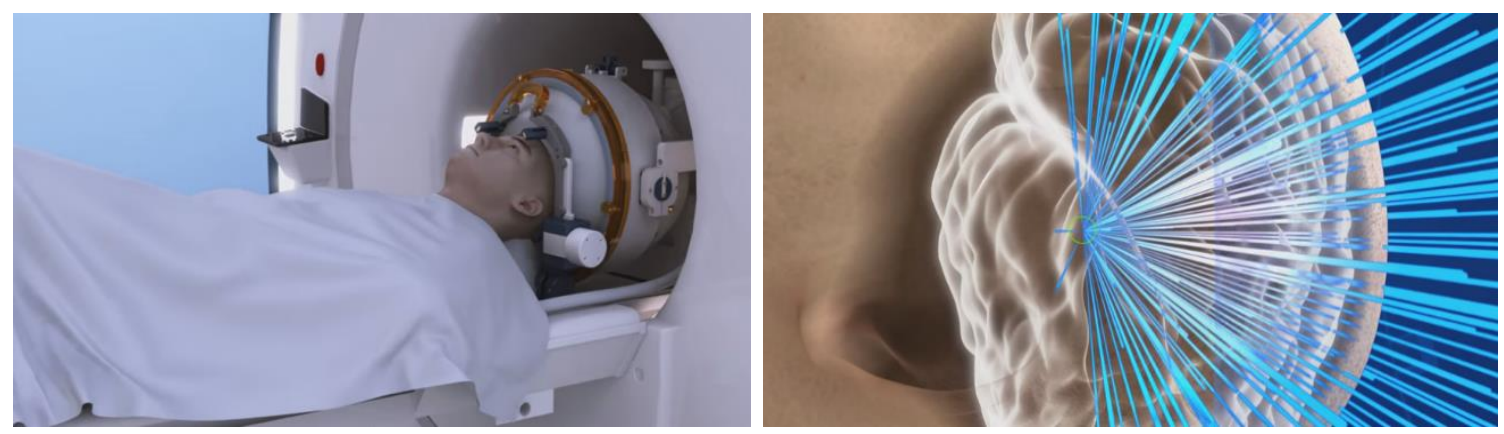

FIGURE 21: MR GUIDED FOCUSED ULTRASOUND, INSIGHTEC.

For essential tremor treatments, Focused ultrasound waves with high intensity target a focal point in the Vim nucleus in the thalamus, small section of the brain that is thought to be responsible for causing tremors. The transducer array consists of 1024 elements to provide enough heat to ablate the targeted tissue during treatment. During the treatment, the patient is fully conscious and lying on the bed of the MRI scanner. The role of MRI is to provide high resolution visualization, planning of the patient-specific treatment, and finally procedure monitoring.

Although the previous example of focusing ultrasound beams inside the skull gives us bright view of the biomedical application of transcranial ultrasound, for imaging purpose however, ultrasound must not only be directed into the brain, but also received after exiting the head. Therefore, all the limitations involved in transcranial focused ultrasound are present, while the new challenges of receiving a very small scattered signal are introduced. As in the case of focused ultrasound therapy, imaging should maximize the energy inside the brain.

\subsection{Transducer Arrays}

The use of acoustical devices have been used for underwater imaging applications since World War I [90]. Ultrasound were first used in medicine in the 1930s [90]. 
Magnetostrictive materials, nickel for example, and piezoelectric crystals, like Rochelle salt and quartz, were the choices of interest of transduction material until the 1940s. The advent of the piezoelectric ceramics, barium titanate and lead zirconate titanate for example, goes back to the World War II, when there was an intense materials research. Diagnostic ultrasound roots back in the late 1960s by introducing electronic sector scanning. A stretched and poled films of polyvinylidene fluoride (PVDF) showed tensile piezoelectricity in 1969. Higher resolution and faster image formation started in the 1970s, when fixed-focus mechanical sector scanners was replaced by linear arrays with electronic scanning. More detailed explanation of transducer technologies and ultrasound imaging history could be found at [91]-[95]. Nowadays, due to the advances in microelectronics and digital signal processing technology, it is possible to easily process large amounts of data from an array of transducers with large number of elements [92], [96]-[103], [90]. Huge research progress has been seen to introduce new reconstruction algorithms, improve and analyze ultrasound images. This is due to the advances in digital data processing systems [90]. However, the accuracy and usefulness of such algorithms highly depend on the quality of the original echo signal, for example, signal to noise ratio, bandwidth, and dynamic range. That is why making the transducer and its associated front-end electronics are the most important part of ultrasound imaging systems. For sending and receiving ultrasonic signals, piezoelectric crystals, polymers, ceramics, and piezocomposite materials [95] have been used for imaging purposes [90]. The phased array transducers has become popular in medical ultrasound imaging to focus and steer the ultrasound beam [90], [92], [96]-[111]. Many of them have been trying to design and develop a two-dimensional phased array transducers to 
symmetrically focus the ultrasound beam to the region of interest (ROI). One of the main considerations of developing an array is half wavelength spacing between the elements in order to avoid unwanted grating lobes in array response [112], [113]. Flexibility and conformity of the array is another factor which should be taken into consideration [105]. While traditionally transducers were based on rigid substrates and not conformal to human body shape [105], [110], [114]-[116], recent works reported flexible ultrasound transducers for implant ultrasound imaging [105]. These factors would be much more important for micro-fabricated ultrasound transducer [105], [117]-[121].

Prior studies have reported the fabrication of a conformal array of transducers and its benefits as an alternatives to rigid multi-element transducers for medical imaging and nondestructive evaluation [122]-[126]. Such arrays can be wrapped around curved body surfaces or objects and provide more angular coverage compare to handheld medical probes. Such features may provide better detection of objects and defects that are not in direct sight lines of rigid transducers. Conformal array may also give us an advantage of elimination of manually rotate and translate handheld probes. Although there are lots of reported fabrication procedures for conformal array of transducers for imaging purpose, no dedicated flexible and conformal imaging system has been reported. This is likely due to the inability to track the precise location of each element, as well as the difficulty in forming an image from an irregular surface. Following is a brief explanation of previously reported fabrication techniques.

In two past studies a passive polymer matrix was used to embed ceramic rods, with reported trade-off between aspect ratio and flexibility [127], [128]. In another study, a sectioned wafer with the etch trenches was refilled with polydimentysiloxane 
(PDMS) to make capacitive microfabricated ultrasound transducers (CMUTs) [126], [128] which reported to be highly flexible but has the lack of robustness because of cracking of the electrical traces bridging the PDMS-refilled trenches [122]. In other recent works rigid piezoelectric materials were packed onto flexible substrates, for example, attaching piezoelectrics materials onto flexible printed circuits [128], transferring piezoelectrics from polydimethylsiloxane (PDMS) templates to flexible substrates by using adhesive films [127], and fixing transducers onto spring-loaded probe matrices [129], [130]. Although some of these fabrication strategies have resulted in flexible devices, they have limitations in uniformity and scalability [122].

\subsection{Image Reconstruction Algorithms}

Since the development of sonar in 1910s, different acoustical imaging techniques have been used for many applications. B-mode imaging is one of those common modes which is based on the sonar principle [131]. B-mode imaging has applications in nondestructive evaluation and medical imaging. This technique is based on the changes in the acoustical impedance function and, therefore, detects differentiation among different media. By the use of an array of transducers [132] or highly focused single-element transducers [133], spatial resolution of about wavelength can be obtained. Amplitude and phase aberrations are the factors which can ruin the image quality [134], but image construction process is both simple and reliable. B-mode imaging is the simplest way of constructing quantitative ultrasonic images. However, it highly depends on the expertise of the operator.

Several techniques have been used to extract features for texture analysis. These techniques includes but not limited to parametric representations of envelope statistics 
[135], [136], first and second order parameters [137]-[139], and wavelet analysis [140],

[141]. However, all these approaches are dependent on the system being used and had limited success. That is why they are not commonly used on medical devices [142].

Ultrasound tomography (UT) is an imaging technique in which quantitative images would be created based on the density and compressibility of the object of interest (OI) from collected ultrasonic data outside the OI. Such information can be used in biomedical diagnosis, like breast cancer and brain imaging, where malignant tissues can be differentiated from healthy tissues [143]. UT is a non-invasive technique as the data is collected outside the OI and there is no need for biopsy. Although this fact seems to be relatively easy to implement for soft tissues, as it will be explained later in this research, our algorithm reconstruct image of the brain by sending/ receiving low frequency signals to/ from inside the skull [1]. In this section, a brief explanation of UT and its different types were explained and compared.

In UT, the object is surrounded by several transducers which can send and receive ultrasound energy (Figure 22). When one of the transducers send the ultrasound energy, all of them would work as receivers and collect this energy. The procedure follows by sending the energy from the second transducer and collected again by all of them. Such a trend would be continued until all the transducers act as transmitters [143]-[147]. It can be done at different frequencies to collect more information of OI [148]-[159]. Background medium, which is known as homogeneous medium, refers to the region of interest without OI and transducers [143]. The choice of background medium is important to minimize the reflection from the OI. In breast imaging, for example, water mostly is chosen as background medium because of water to the relevant physical 
parameters of breast tissues. Therefore, much of the ultrasound energy penetrates into the breast tissues and, as a result, extracting more information.

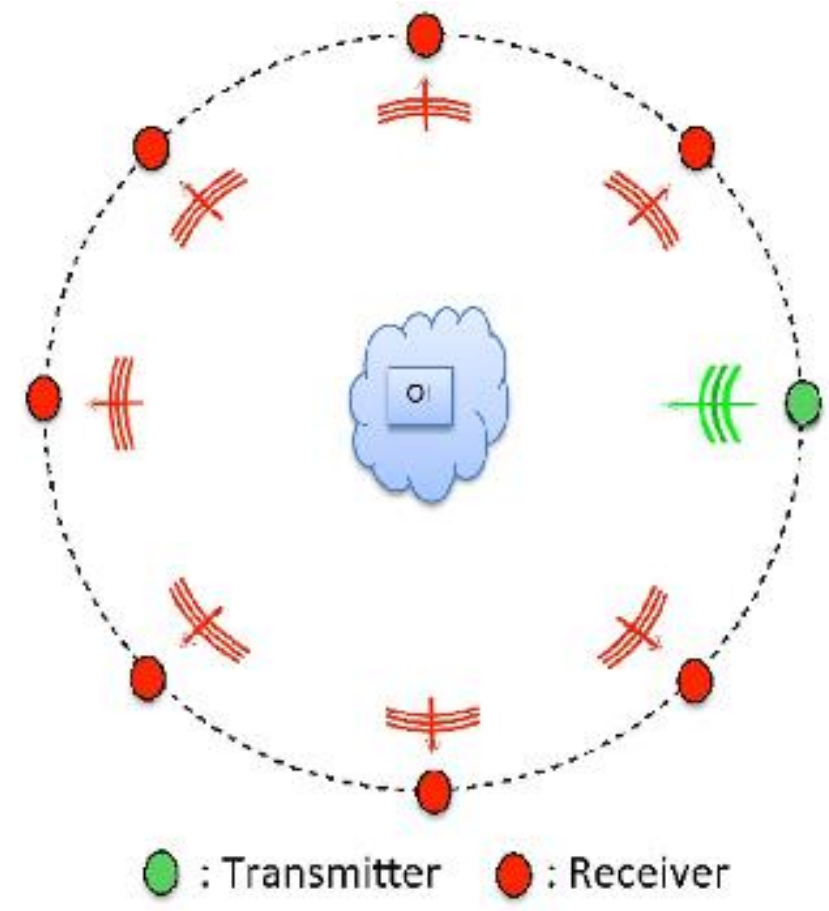

FIGURE 22: ULTRASOUND TOMOGRAPHY SETUP IN WHICH THE OBJECT OF INTEREST (OI) IS ENCLOSED BY AN ARRAY OF ULTRASOUND TRANSMITTERS/RECEIVERS. TAKEN WITH PERMISSION [143].

When the transmitter sends the ultrasound wave to the imaging domain (OI and background medium), it would be scattered and then collected by the receivers. To get the scattered field information, the measurements are first performed in the background medium in the absence of the OI and refers to incident field measurement. Then the same procedure would be applied in the presence of the object (total field data). Scattered field data is resulted from the subtraction of incident field data from the total field data. These scattered field data is then used for UT problem which can later be mathematically formulated as an inverse scattering problem which is a nonlinear problem [143], [148], [149], [157], [158]. 
The low frequency conformal array designed in this study as well as the image reconstruction algorithm it is based around are completely new. As such there is no history of such use in the literature. Following is a brief literature review of the applications of UT and its comparison to other image modalities presented.

Biomedical UT has been highly applied in the studies of breast cancer detection [143], [149], [160]-[164]. Breast cancer is ranked first in the number of cancer occurrences in women and is considered as the second most common occurring cancer across the world. It is predicted that around 1,152,000 new breast cancer incidents would be reported each year and around 411,000 patients die [165]. Therefore, imaging techniques which provide high resolution (to distinguish between benign and malignant tumors) for early detection of the breast cancer would be highly crucial. Current imaging methods are X-ray mammograms, Magnetic Resonance Imaging (MRI), X-ray Computed tomography (CT) and conventional ultrasound imaging [166]. But new research studies have demonstrated the use of microwave and ultrasound tomography using inverse scattering methods as an alternative to current imaging techniques.

MRI is high resolution imaging system which can be used for detection of breast cancer or tumor in the brain. But the cost and the volume of the system are the major drawbacks compare to ultrasonic system which is portable and much cheaper (Figure 23). It also needs the use of contrast agents for breast imaging [162], [167]. X-ray is based on the photon absorption in different tissues with different density. As x-rays are ionizing radiation, it is suggested to be avoided [168]. Figure 24 shows a typical CT scan used in hospitals. 


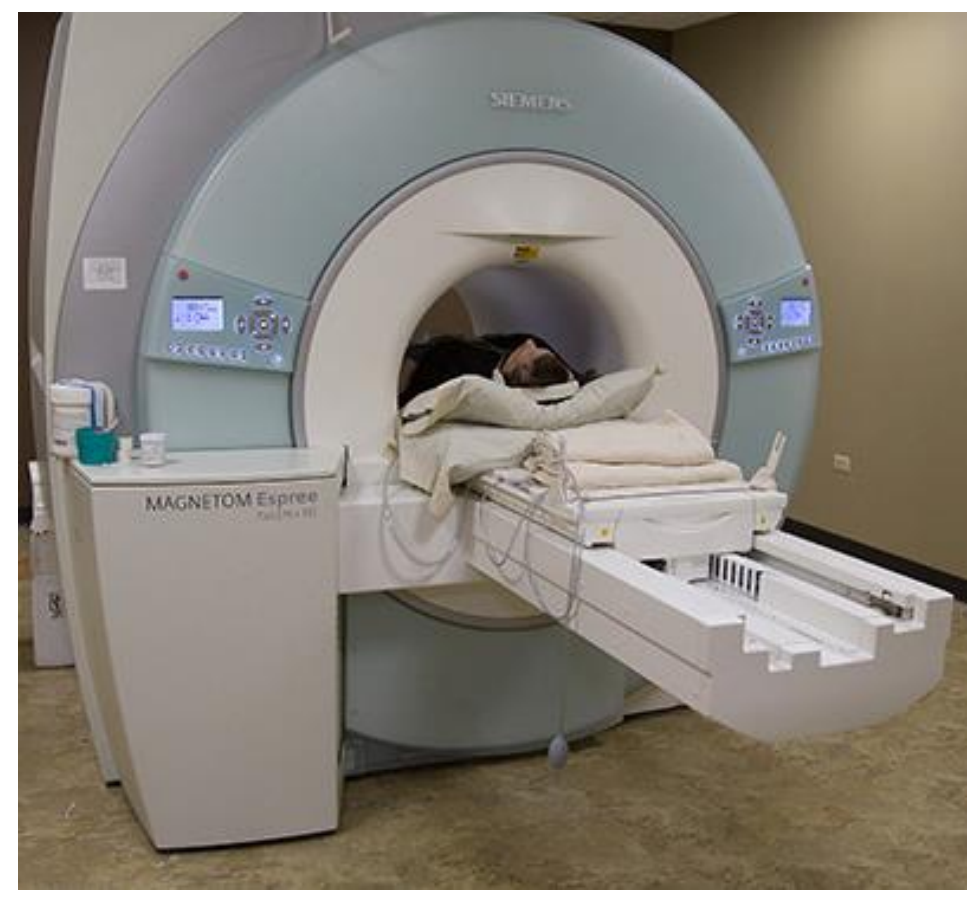

Figure 23: MAGNETIC RESONANCE IMAGING (MRI).

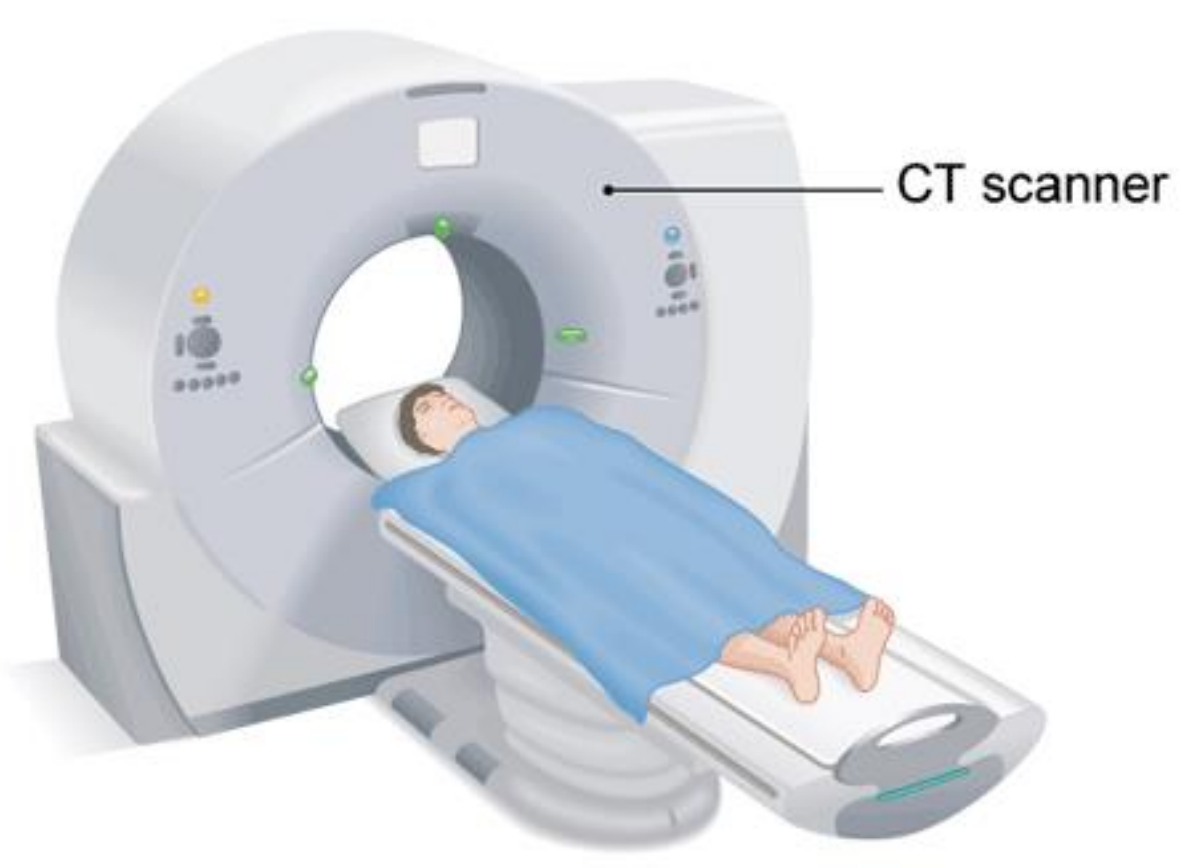

FIGURE 24: COMPUTED TOMOGRAPHY (CT). 
Ultrasound imaging was introduced by the invention of the supersonic reectoscope [169], [170]. Ultrasound sonography and ultrasound tomography techniques were then introduced for image construction of the object of interest. The main problem of sonography is that it is operator dependent. To overcome such a problem a technique which is not dependent on the operator should be taken into consideration [162].

Ultrasound Tomography (UT) which is a quantitative imaging system has much potential for biomedical imaging. As mentioned before, ultrasonic waves would be sent to OI and scattered energy would be detected for image reconstruction. There are three main approaches in UT: 1- Diffraction tomography [143], [160], [162], [171]-[173] 2Time of flight tomography [162], [174], [175] and 3- full-wave inversion algorithms [143].

The approach in this study seeks to overcome skull attenuation by applying sufficiently low frequency beams to penetrate maximum energy through skull and designing a transducer array to receive the scattered signals all around the imaging plane. This cannot be done by conventional amplitude time of flight imaging. In B-scan, for example, the time-of-flight (or travel time) of the sound energy is displayed along the vertical axis and the linear position of the transducer is displayed along the horizontal axis; therefore, the ultrasound scanner should be capable of determining two important things: time of flight and echo strength. Then the ultrasonic scanner can locate which pixel in the image to light up and to what intensity. In addition, Different ranges of frequencies with the trade-off between spatial resolution of the image and imaging depth would be used to reconstruct the image (see Chapter 2 for details). However, as the ultrasonic imaging system is designed to get 2D imaged of the brain, it is impossible to 
use the conventional imaging mode. The skull highly attenuates and scatters the signal (see Chapter 2 for details) and therefore, the collected signal cannot provide neither of information of time of flight and detectable echo strength.

The new technique of ultrasound diffraction tomography and inverse scattering is based on a single low frequency ultrasound which would be applied to any arbitrarilyshaped curve of sources and receivers [1]. Therefore, for intended use in this project of brain imaging, it allows us to provide coupling to the head by attaching the transducers to the skull skin. Moreover, using a single frequency ultrasound would allow us to deliver more energy at a single frequency and to enhance receivers' sensitivity for collecting the scattered signals at that frequency.

The way of collecting data in diffraction tomography technique is called full matrix capture (FMC). In FMC, one of the elements of the array would fire the signal and all of the elements would detect the received scattered signal. This would be repeated till all the elements would fire the signal once (see Chapter 2 for details). As the algorithm is based on a single frequency, the resolution would be increased by transmitting data from different angles of transmitting the signal. Therefore, an array of transducers is made to satisfy all the requirements needed for brain imaging by applying the new image reconstruction approach [1]. The array is flexible to provide optimal coupling to the nonsymmetrical shape of the human head (see Chapter 4 for details). It also consists of elements with highest sensitivity to their resonance frequency. As the reconstruction algorithm works only with single frequency, we do not need further backing for the transducers. Therefore, the transducers can deliver more energy at a single frequency and have high sensitivity for that frequency to receive the scattered signal in the brain. In 
addition, this would be a phased array of transducers tighten around a plane of the head (to be imaged later) to perform FMC from different angles of the head. 


\section{CHAPTER IV}

\section{METHODS}

Chapter 4 begins with Section 4.1, which introduces the materials used in the transducers. This is followed by Section 4.2, which gives a broad view of the design. Four design aspects, including the transducers' dimensions and structure, PC board design, connecting PC board to PZT, the housing of the transducers, and their electrical connections are discussed in this section. The experimental setups for testing the functionality of each element as well as evaluation of their isolation from nearby elements is explained in Section 4.3. Finally, the steps involved in designing an expander for the data acquisition system to cover 512 elements are explained in Section 4.4.

\subsection{Material}

The properties and parameters of transducers are determined based on the specific tasks for which transducers are going to be used. Factors like resonance frequency and vibration mode depend on the geometry of the piezoelectric transducers, while the band width and the acoustic impedance depend on the choice of materials. In recent years, the 
most common source of transducer ultrasonic waves has been from piezoelectric materials, which transform electrical signals to mechanical vibrations. Crystalline materials have been used as well, and while they have high quality factors, they also operate over small band widths. Piezoelectric ceramics, are relatively cost-effective and can be cut or shaped into different geometries much easier. For the applications of high frequency ultrasound, crystalline or ceramic films can be used.

Piezoelectric composite materials consist of an active piezoelectric component and a passive piezoelectric matrix (typically epoxy resin or other polymers). With this combination, they have the piezoelectric properties of ceramics as well as the low density and softness of polymers. Piezoelectrics have high electromechanical coupling coefficients, low density, and, therefore, low acoustic impedance. In addition, these composites are highly elastic compared to the bulk ceramic materials [176]. Further effective properties of 1-3 composites in addition to methods of construction are explained at [176].

The electromechanical portion of the transducer used in this project is formed around a $20 \times 20 \mathrm{~mm}$ composite plate (Smart Material Corp), made of $65 \%$ lead zirconate titanate (PZT) 1-3 piezoelectric ceramic fibers that act as active rods. They are randomly arranged and then filled in with epoxy. The 1-3 fibers are $250 \mu \mathrm{m}$ thick, and the random placement in the cross-section creates reduced acoustic impedance (22 MRayl) and a high coupling coefficient (0.61-0.68). Furthermore, 1-3 fiber composites with random element distribution resulted in increasing spurious mode suppression because of the random spacing [177]. Figure 25 explains how such transducers are assembled. 
The transducers are layered with electrically conductive surfaces (copper tin, $\mathrm{CuSn}$ ) on both sides. These electrode surfaces conduct electric signals to and from the transducer. The role of PZT in ultrasonic transducers was evaluated in [36], [178].

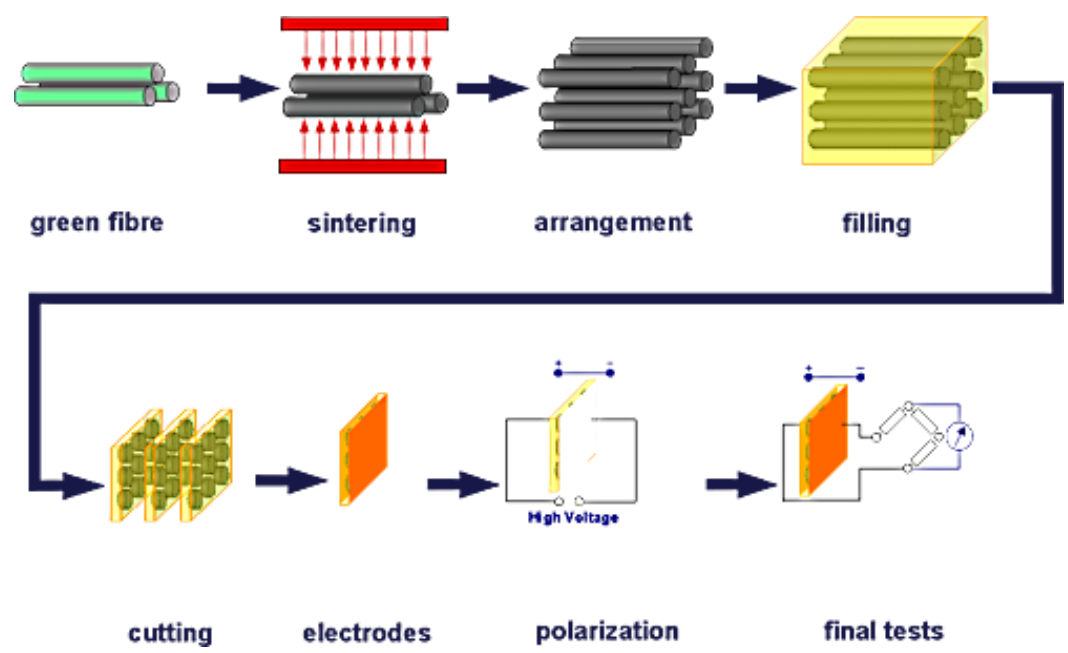

FigURE 25: A GRAPHIC SHOWING HOW PZT TRANSDUCERS ARE CREATED. USED WITH PERMISSION. TAKEN FROM [177].

\subsection{Design}

The most important part of the project was the design section. The array should be designed in a way that provides the flexibility to be easily wrapped around the head for acoustic coupling, being adjustable for different sizes of the human skull, and provide electrical connectivity for around 500 tiny elements without or with minimum cross-talk. The step by step process of designing the array will be explained later. In the next section, the way of sub-dividing the transducers to smaller elements for having around 500 elements of transmitters/receivers is explained. This would be followed by the explanation of designing PC boards and connecting them to the PZTs for electrical 
connectivity. Finally, the way of designing a flexible housing for transducers are explained in details.

\subsubsection{Transducer}

As mentioned in the introduction, the design requirements are for an array of transducers that consists of around 500 elements of sources and receivers surrounding the desired plane to be imaged. Instead of using 500 separate transducers, the $20 \times 20 \mathrm{~mm}$ plates were further divided into 19 separate transmitting and receiving elements with a center-to-center spacing of approximately $1.05 \mathrm{~mm}$. This was achieved by using a dicing saw to make $1 \mathrm{~mm}$ deep cuts along the length of only one of the electrode faces, creating approximately $800 \mu \mathrm{m}$ wide elements (Figure 26). The result of using these $20 \times 20 \mathrm{~mm}$ transducers to make the conformal array, however, is that the rigidity and flexibility of the array is determined by the plates.

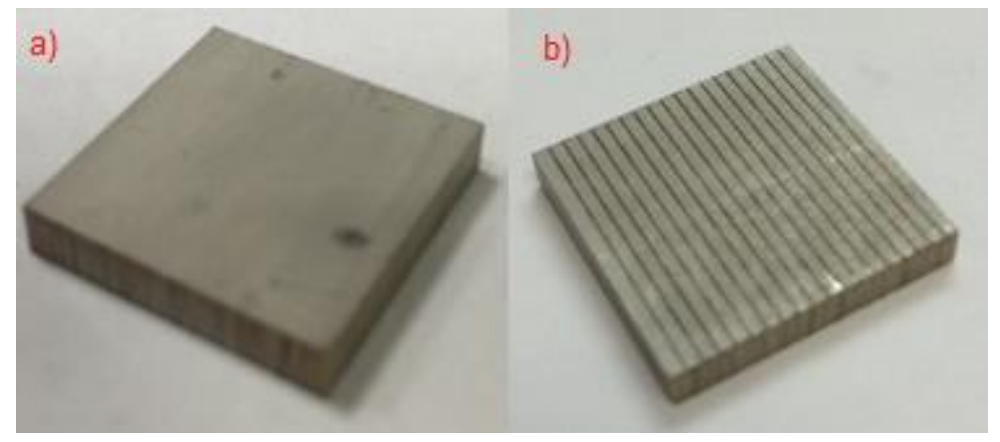

FIgURE 26: A) A SINGLE 20X20 MM TRANSDUCER B) THE PLATE WAS SUBDIVIDED INTO SMALL ELEMENTS.

After testing 3 transducers and determining the efficacy of such a layout, the rest of the transducers was sent to American Precision Dicing, Inc, for professional cutting. The Results section will discuss this in more details. In summary, these small cuts adequately separate the elements in terms of sending and receiving pressure signals with 
minor cause of vibration of nearby elements. This explains why 1-3 PZT composite was chosen over PZT crystal; the use of crystal would not allow for mechanical isolation of subdivided elements.

\subsubsection{PC Board}

One of the biggest challenges in designing the array was wiring each element of the transducers for electrical signal sending and receiving. As mentioned before, each transducer is $20 \times 20 \mathrm{~mm}$ plate with 19 elements. Therefore, 19 elements should be wired for each transducer. In addition, the curie temperature of ceramic plates is about $100^{\circ} \mathrm{C}$. Therefore, there are two main limitations of providing strong electrical connectivity for each elements: 1- having small space for connecting wires to each element and 2difficulty of hot or flow soldering.

In order to make strong and simple connections to the transducer elements, a double-layer PC board was designed, fabricated, and then connected to each PZT by using silver conductive epoxy (E4110, by Epoxy Technology, Inc). The design of the PC board was done in SolidWorks ${ }^{\circledR}$ and Eagle ${ }^{\circledR}$ and then it was sent to a prototype board manufacturer (OSH Park) for fabrication. The full, final assembly should be as light and pliable as possible, so the array can easily be wrapped around the head for a series of scans and then removed (Figure 27). Without the PCBs, each plate is about 6.2 grams, and increases to 9.5 grams once the PCBs are attached. The PC board is a standard, 1/16" thick, double-sided board with two layers of 1 oz. copper laydown on non-conductive FR4 substrate. The PC board would be connected to the transducer (the surface with straight, isolating cuts) via conductive epoxy on one side, and to thru-hole ribbon cable connectors on the other side. The ribbon cables should be shielded to protect the data 
signal from any outside noise, which adds significantly to their weight. The overall weight of each transducer with connected board, connectors, and ribbon cables increases to be around 32 grams.
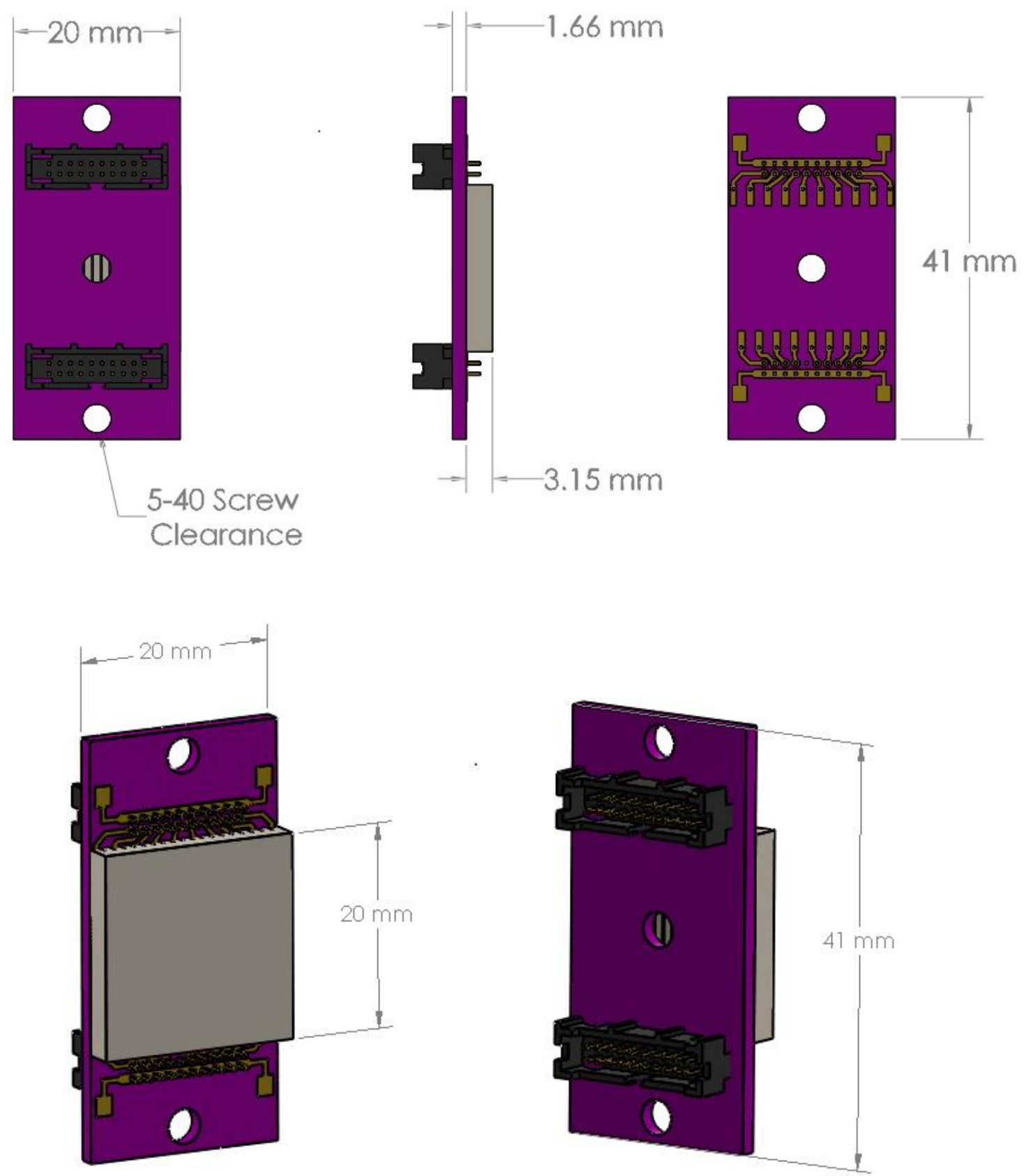

FIGURE 27: THE UPPER THREE PICTURES SHOW DIFFERENT VIEWS OF THE PC BOARD WITH CONNECTORS ATTACHED TO IT. THE BOTTOM TWO IMAgES SHOW HOW THE PZT IS CONNECTED TO A PC BOARD. THE MIDDLE HOLE IS TO LATER INJECT A LOW-VISCOSITY EPOXY. 


\subsubsection{Connecting PC Board to PZT}

Because of the 1-3 composite material's relatively low thermal budget, along with the precision needed to properly isolate each element, hot soldering connections is impractical. Therefore, in order to attach the PC board to PZT, silver conductive epoxy was used. It was chosen based on the recommendations received from specialists at Epoxy Technology, Inc. In order to do this, tiny epoxy dots were carefully put at one end of each element of the PZT and the PC board was attached. This was done through the use of a pressure syringe pump (EFD Ultimus air-powered dispenser) connected to a 30 gauge needle (Figure 28). The size of dots was shown in Figure 29. Parameters of pressure, air pulse time, and dispensing needle size were tested until the epoxy dots were averaging between $350-450 \mu \mathrm{m}$, which would fit safely inside each element without the risk of accidentally connecting to the adjacent elements.

This specific conductive epoxy was also chosen because of its relatively low curing temperature. The connected PC board and PZT should be at $60^{\circ} \mathrm{C}$ for six hours for the epoxy-curing process. After that, testing was conducted on each element to determine that it was not connected to other nearby elements. The resistivity of each dot was measured; results showed that the resistivity of the dots was between 0.1 and $.15 \Omega$.

As 19 small epoxy (EPO-TEK® E4110, Appendix A) dots (one on each element) cannot provide enough bondage to hold the PZT and PC board together, a very low viscosity, “wicking” epoxy (Loctite® \#493 cyanoacrylate, Appendix B) was injected between the surfaces of PC board and PZT. To ensure comprehensive coverage of the bonding agent, a hole was made on the back of the PCBs for injection purposes. This 
forms a non-conductive bond strong enough for the PZT-PC board connection to survive the bending and twisting nature of the full flexible array (Figure 27).

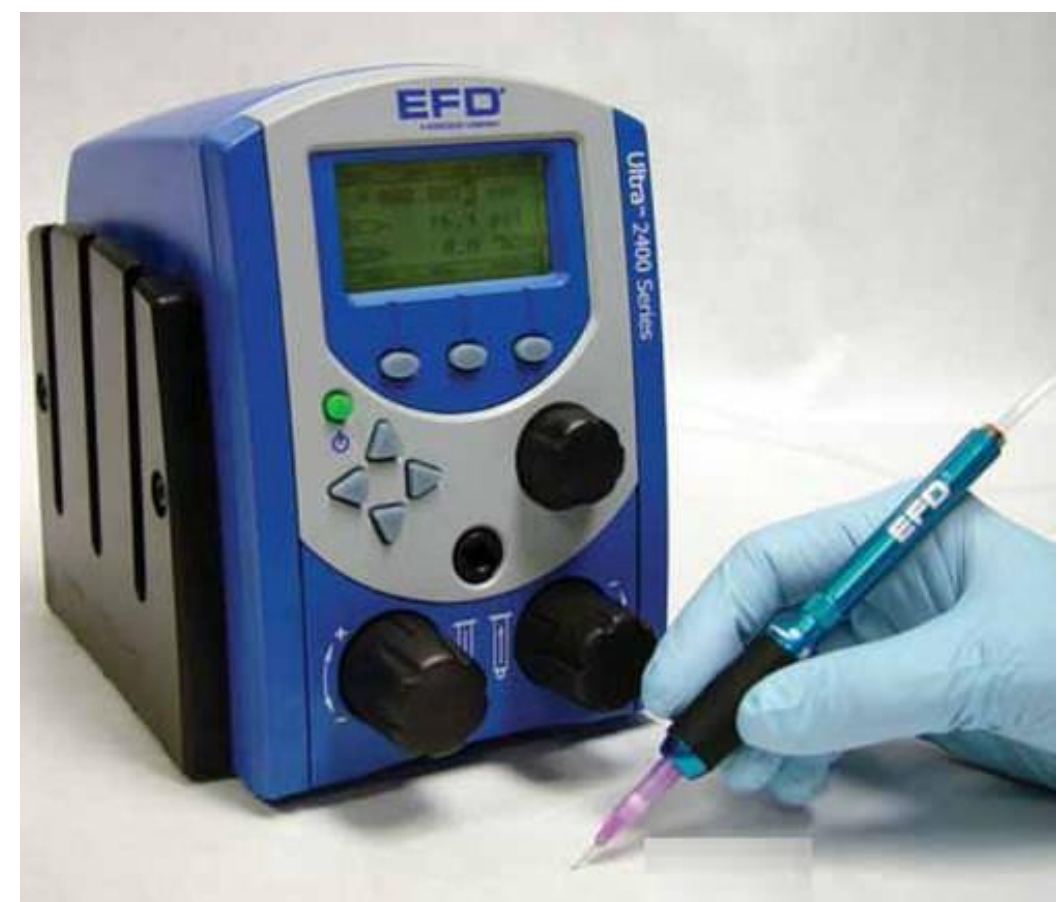

FIGURE 28: EFD ULTIMUS AIR-POWERED DISPENSER. AIR PRESSURE, AIR PULSE TIME, AND DISPENSING NEEDLE SIZE ARE CONTROLLING PARAMETERS ON PUTTING THE CONDUCTIVE EPOXY DOTS.

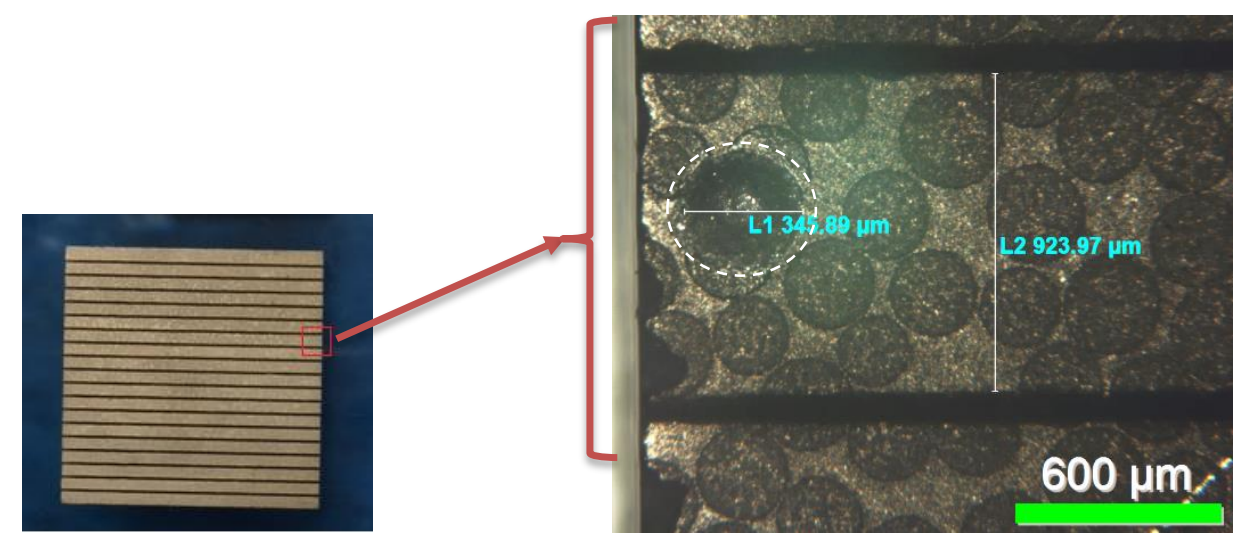

FIGURE 29: CONDUCTIVE EPOXY ON THE PZT BEFORE CONNECTING THE PZT TO PC BOARD. FOR ELECTRICAL CONDUCTION, EACH ELEMENT WAS CONNECTED TO THE PC BOARD USING CONDUCTIVE EPOXY DOTS.

THEREFORE, TOTAL OF 19 DOTS WERE CONSIDERED FOR EACH PZT, PLACED AT THE VERY END OF EACH ELEMENT. 


\subsubsection{Housing}

The array housing with transducers and PC boards is composed of a 3D printed material: a proprietary flexible, acrylic-based polyjet material called TangoPlus, which is made by Stratasys, Inc. A concept image of a small segment of the full array can be seen in Figure 31. To provide a good matching layer between the transducers and the housing, the same $3 \mathrm{D}$ printed polyjet material that was used to create the array housing was bonded to the surface of the transducers. During normal printing, this material cures from exposure to ultraviolet light. To bond the transducers to the array housing, uncured, liquid resin version of the polyjet material was injected between the transducer surface and the housing. Then, by applying UV light, the liquid resin cures and forms both a strong bond between transducers and the housing, and a flexible, homogeneous matching layer that facilitates sending and receiving ultrasound signals.

The thickness of this matching layer was calculated according to the $\lambda / 4$ of the resonance frequency of the PZT. The speed of the sound in the material was measured relative to the water (as the reference) by applying the insertion technique [179]. This technique is based on a relative measurement method. Using this method, a reference is used to study the transmission of longitudinal ultrasonic waves through a solid media embedded in an aqueous environment [180]. The sound velocity is then calculated from equation (5).

$\frac{1}{c_{t}}=\frac{1}{c_{w}}-\frac{\Delta t}{\Delta x}$ 
In this equation, $\Delta t$ is the temporal shift between the pulse transit times with and without samples; measured by an oscilloscope, $c_{t}$ is the acoustic velocity of the material, $c_{w}$ is the acoustic velocity of the water (reference), and $\Delta x$ is the length of the material.

As shown in Figure 30, a tank with two transducers directed at each other from parallel walls of the tank is filled with water. A piece of polyjet material with known length was placed between the transducers. A one $\mathrm{MHz}$ (resonance frequency of the transducers, Panametrics-NDT, A303S, Waltham, MA, USA), 1-cycle pulse was transmitted and received with and without the sample in place. Then, the temporal shift $(\Delta t)$ between the pulse transit times with and without sample was measured using an Oscilloscope.

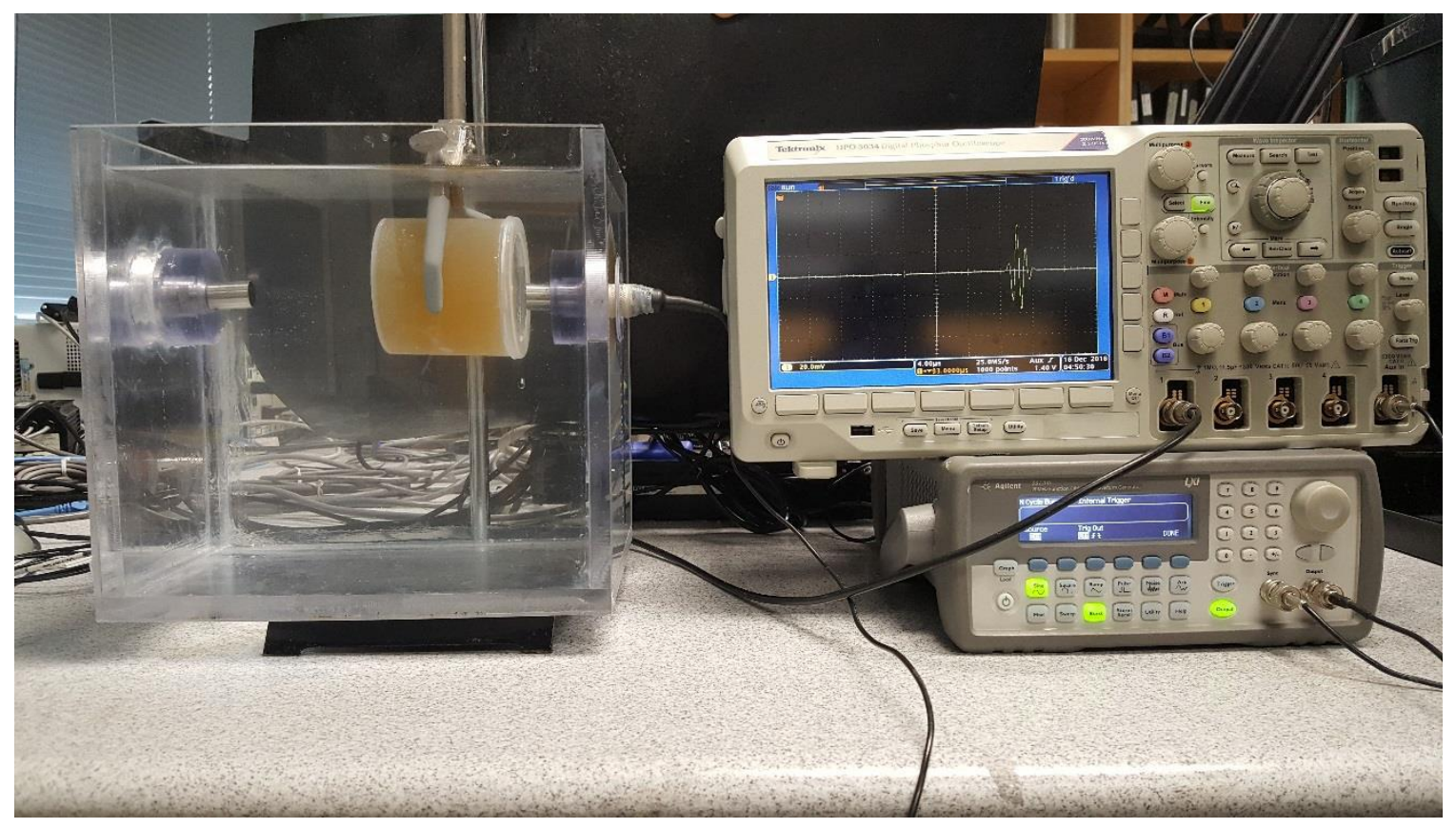

FIGURE 30: CALCULATING ACOUSTIC VELOCITY USING INSERTION TECHNIQUE. THE EXPERIMENTAL SETUP SHOWS HOW THE VELOCITY OF THE SOUND IS MEASURED THROUGH THE POLYJET MATERIAL TO LATER CALCULATE THE OPTIMAL THICKNESS OF THE MATCHING LAYER. TAKEN WITH PERMISSION FROM [181]. 
By measuring the speed of the sound as it moves through the headband material, the matching layer thickness should be approximately $0.536 \mathrm{~mm}$ (Figure 31 and 32).
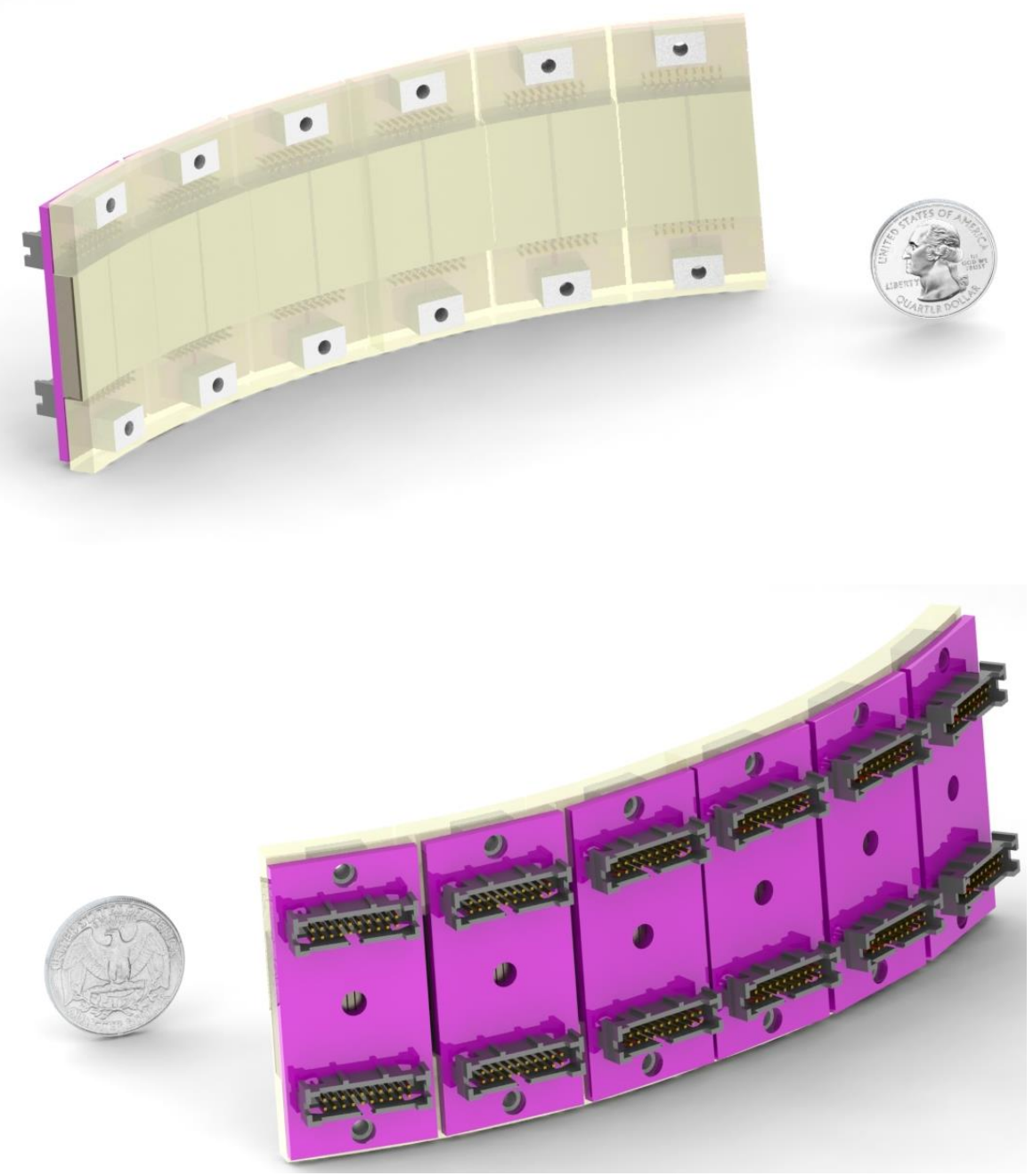

Figure 31: EARLy Flexible HeAdBANd DESIgN. 


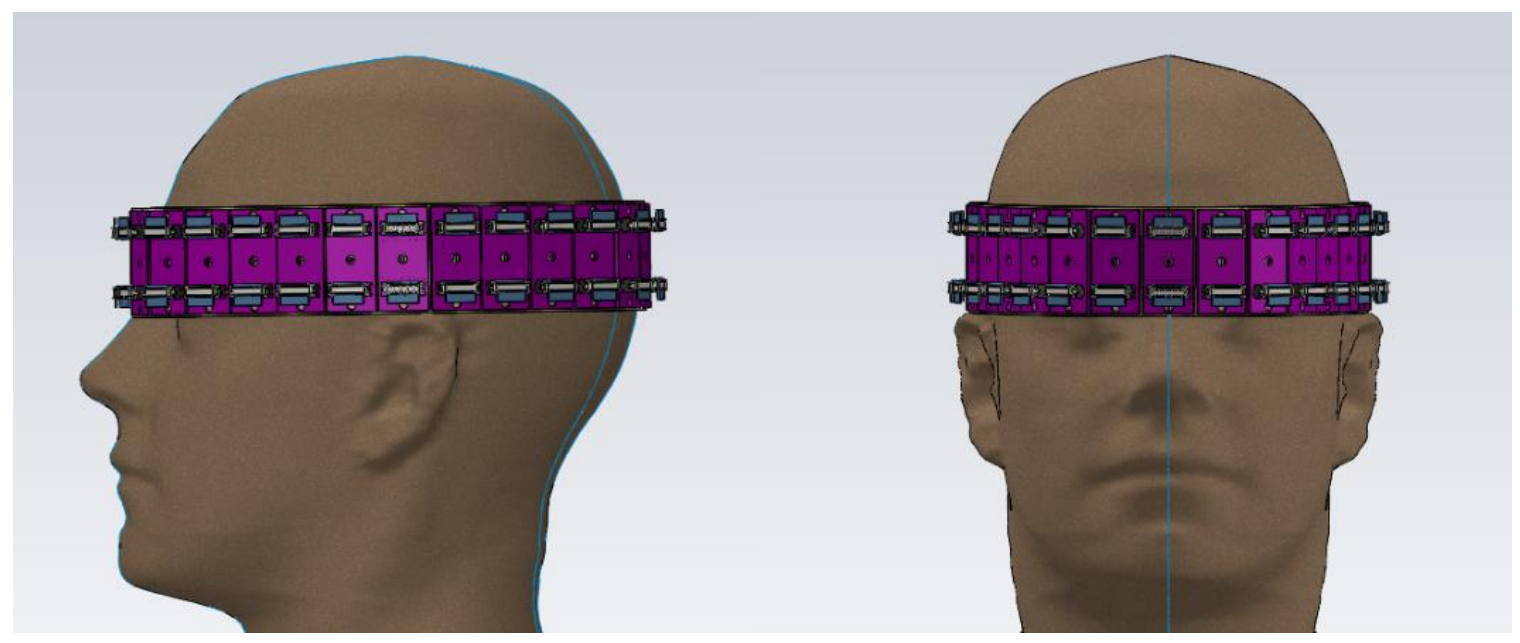

FigURE 32: POSITION OF THE TRANSDUCERS ARRAY ON THE HUMAN SKULL.

\subsubsection{Electrical Connection}

As mentioned in section 3.2.3, in order to provide electrical connections to each elements of the transducers plates, tiny aluminum conductive epoxy was used to bind the elements to the PCB (Figure 29). The PCBs, on the other side, are connected to two thruhole connectors (Figure 27). These connectors are attached to shielded ribbon cables (Figure 33). To eliminate noise within the cables, the PCBs and the connectors are designed in a way that every other wire in the ribbon cable is grounded.

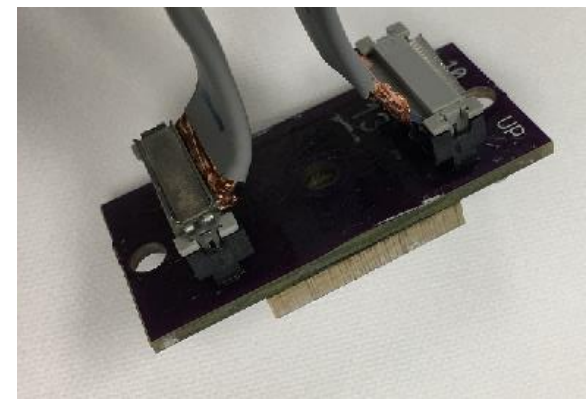

FIGURE 33: ELECTRICAL CONNECTION OF A SAMPLE OF A TRANSDUCER PLATE CONNECTED TO PCB AND RIBBON CABLES. 
Each ribbon cable has 20 bundled, but separate wires. Therefore, each ribbon cable would support up to 10 elements. As there are 19 elements on each plate, the PCB was designed in a way that it supports two connectors and therefore, 19 elements.

These ribbon cables should be then connected to the data acquisition system for collecting required data. However, this presented another challenge as the array interface channels of the acquisition system supports only pin connections (Figure 34).

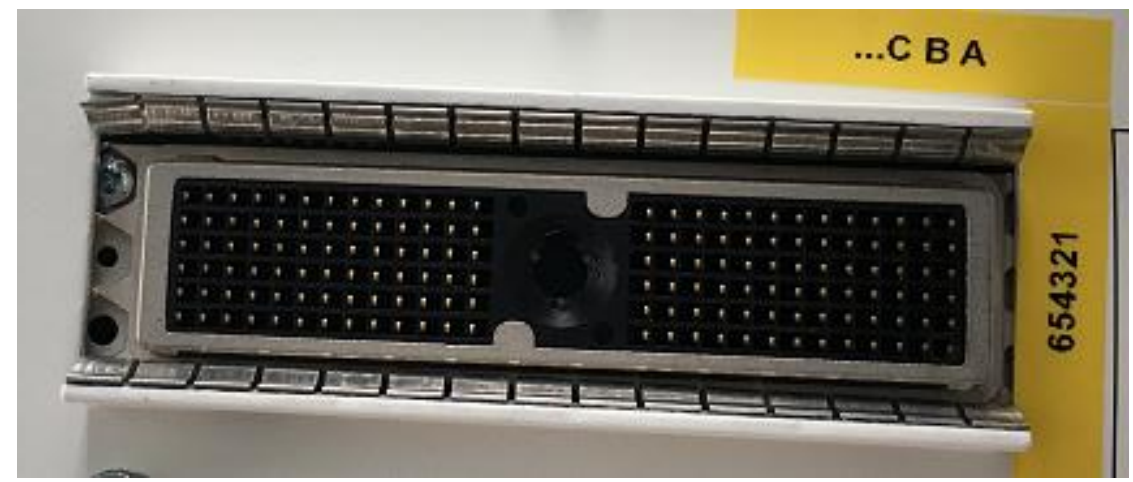

FIGURE 34: ARRAY INTERFACE VIEW OF THE CONTROL AND ACQUISITION SYSTEM.

To connect the ribbon cables to the system, two PCBs were designed to be the interface between the array interface channels and the ribbon cables. These two PCBs would be connected by thru-hole vias to a male plug header with contacts protruding from its rear face (Figure 35 and 36).

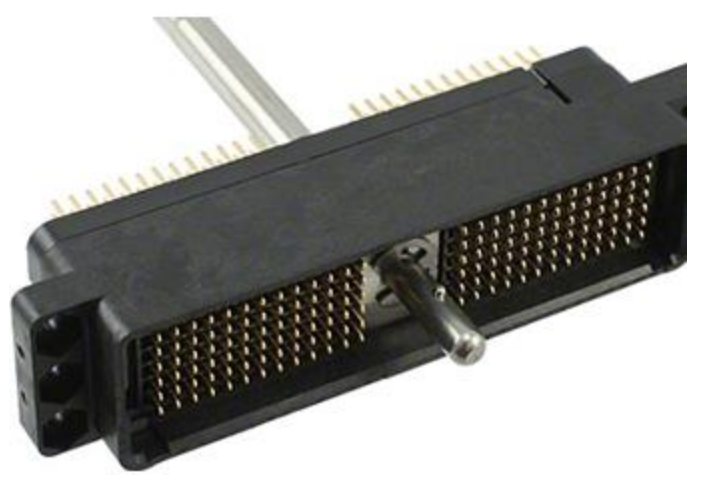

FIGURE 35: A MALE PLUG HEADER WITH CONTACTS STICKING OUT OF THE BACK OF IT. ITT CANNON ZIF CONNECTOR, DL1-156PW6A. 
The PCBs were designed in a way to be mounted on the headers by finely pressfitting them to the header pins (Figure 36). While affixing the boards in this fashion was not ideal compared to soldering, it did provide secure electrical thru-hole connections for the connectors. This is an area of the project that, in future versions, should be optimized for electrical signal transfer.

Mounted PCB on the ZIF connector provides connections for up to 6 transducers. Every two connectors are connected to 19 elements. Therefore, the new interface can provide 114 connections to the channels using ribbon cables.

The test results showed that by connecting non-shielded ribbon cables to the device, there would be a severe crosstalk between the elements. However, by grounding the shielded ribbon cables, the effect of crosstalk could be possibly decreased.

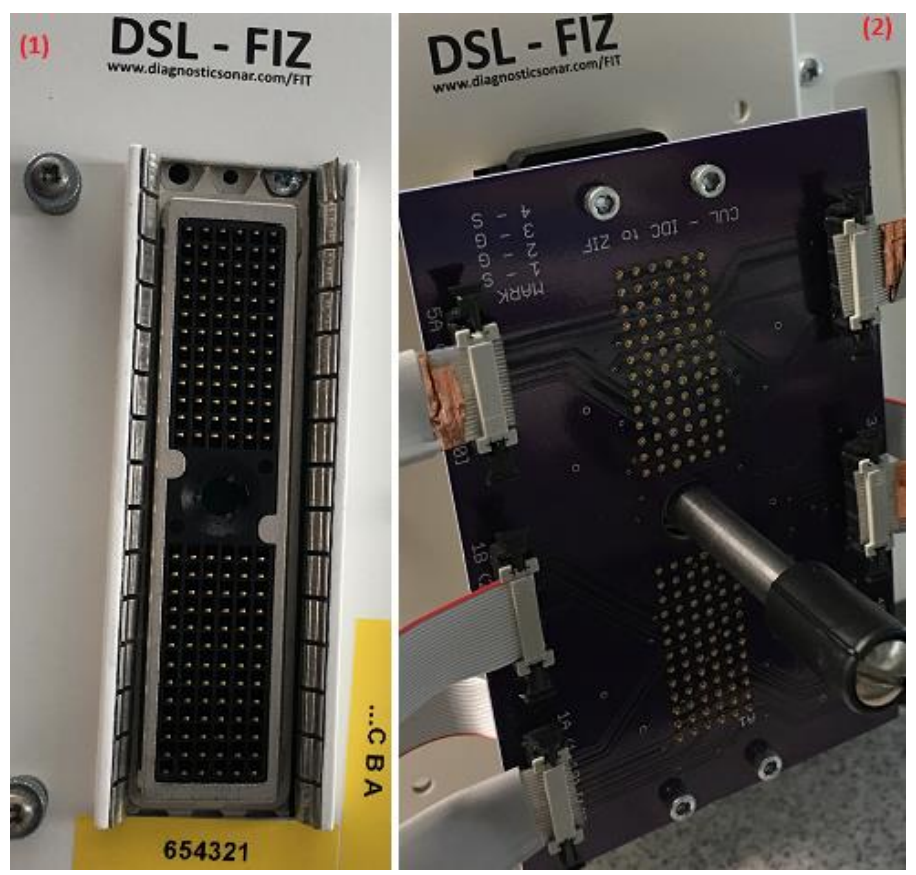

Figure 36: ConNeCting PCBS TO THE MALE PLUG HEADER to PROVIDE INTERFACE CONNECTIONS BETWEeN THE CONNECTORS AND THE ARRAY INTERFACE CHANNELS OF THE SYSTEM. 1) ARRAY INTERFACE OF THE DATA ACQUISITION SYSTEM. 2) PCB MOUNTED ON THE DL CANNON ZIF CONNECTORS TO PROVIDE ELECTRICAL CONNECTIVITY BETWEEN THE RIBBON CABLES AND DATA ACQUISITION SYSTEM. 


\subsection{Testing}

Transducer characteristics were measured by using an experimental system shown in Figure 37 [182].

The large rectangle shows a water tank in which, the measurements were performed inside. Transducers $(\mathrm{T})$ were rigidly mounted to an acoustic window in the wall of the tank with propagation along the long axis of the tank.

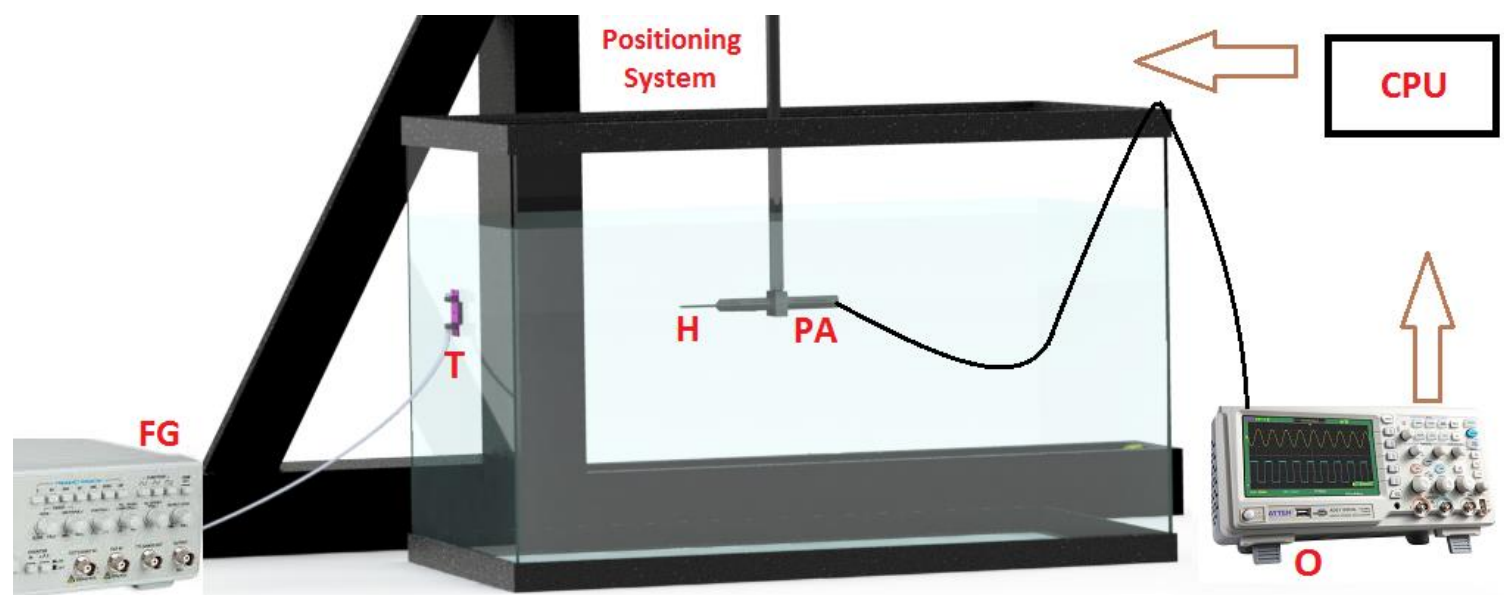

FIGURE 37: EXPERIMENTAL SETUP FOR MEASURING THE PRESSURE PLANE IN FRONT OF THE TRANSDUCER. TAKEN WITH PERMISSION FROM [182].

Scanning was done using a Precision Acoustics $0.5 \mathrm{~mm}$ PVDF hydrophone $(\mathrm{H})$ attached to a Parker 3D stepping, motor-guided positioning system. The scanned plane is perpendicular to the acoustic axis. The electrical signal was produced and sent by a function generator $(\mathrm{FG})$ to the transducer. Then, the pressure signal was received by the hydrophone and amplified by a Precision Acoustics (PA) submersible preamplifier. Finally, the signal would be recorded by an oscilloscope $(\mathrm{O})$. The position of the hydrophone and data acquisition would be controlled using a computer (CPU). 
Three transducers were tested in this experiment. Each had 19 elements as described above, resulting in a total of 57 elements to be tested. For each measurement, the hydrophone scanned a plane of $60 \times 60 \mathrm{~mm}^{2}$ at steps of $1 \mathrm{~mm}$. For each plane, the time trace from oscilloscope transferred to a PC and then, by applying a spatial Fourier transform, the amplitude and phase information were acquired at the temporal frequency of $0.5 \mathrm{MHz}$, which is the resonance frequency of the transducers. When measuring the pressure field, if the hydrophone comes too close to the surface of the transducer, it is going to disturb the field and the measured field would not be accurately represented. Thus, the planes were measured at $50 \mathrm{~mm}$ in front of each element, to be later backprojected at the surface of the transducer by using a spatial planar projection technique introduced at [182]. The projection algorithm can be applied for linear acoustic fields. Linear approximation is an appropriate method for evaluating transducers [183].

For a field which is temporal-harmonic, the temporal dependence can be separated and the pressure is written as follows:

$P(r, t)=\tilde{P}(r) e^{i w t}$

Therefore, the wave equation becomes:

$$
\left(\nabla^{2}+\frac{w^{2}}{c^{2}}\right) \tilde{P}(r)=0
$$

Equation (7) is the Helmholtz equation in which $w$ is the angular frequency, $c$ is the speed of the sound and $P$ is the pressure field.

We can then write the Helmholtz equation in Cartesian coordinates by substitution of the following 2D Fourier integral: 
$\tilde{P}(x, y, z)=\frac{1}{2 \pi} \iint p\left(k_{x}, k_{y}, z\right) e^{i w t} e^{i k_{x} x} e^{i k_{y} y} d k_{x} d k_{y}$

Then by putting equation (8) into (7) we would have:

$\frac{\partial^{2} p\left(k_{x}, k_{y}, z\right)}{\partial z^{2}}+\left(w^{2} / c^{2}-k_{x}{ }^{2}-k_{y}{ }^{2}\right) p\left(k_{x}, k_{y}, z\right)=0$

By solving equation (9), which is a second order differential equation and considering boundary conditions at $z_{0}$, we will have equation (10) which is an advance solution for equation (9).

In order to project the field measured at $z_{0}$ in front of the transducer, to an arbitrary field at distance $\mathrm{z}$, the equation 4 can be applied [182]:

$p\left(k_{x}, k_{y}, z\right)=p\left(k_{x}, k_{y}, z_{0}\right) e^{i\left(z-z_{0}\right) \sqrt{w^{2} / c^{2}-k_{x}^{2}-k_{y}^{2}}}$

This equation says that the field recorded in plane $z_{0}$ is related to the field at any other plane $z$ by a simple transfer function on the right side of the equation (10). In this equation, $w$ is the angular frequency, $\mathrm{c}$ is the sound speed in the medium, and $k_{x}, k_{y}$ are spatial frequency coordinates. By taking the inverse Fourier transform of the $p$ at distance $z$, the pressure field at $z$ may be obtained.

The algorithm was then applied to the newly fabricated transducers. Figure 38 shows a comparison between projected and directly-measured data. 


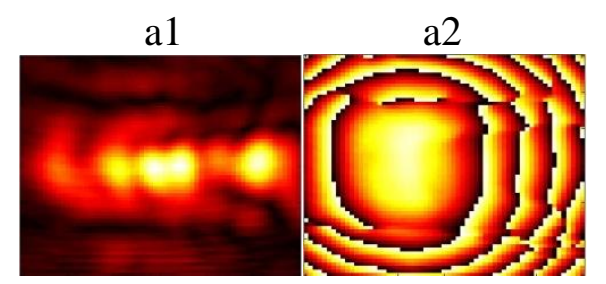

b1

b2

c1 c2

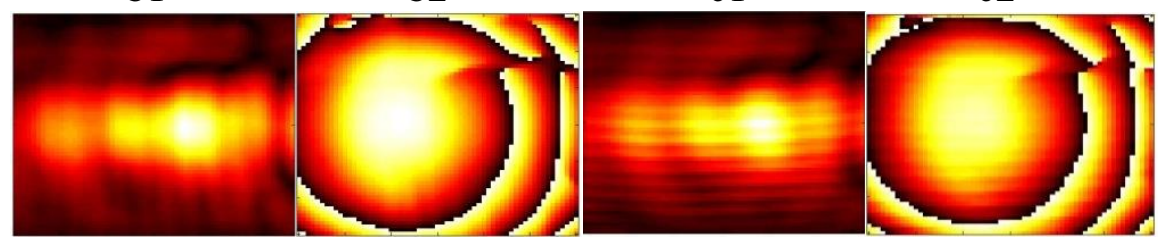

FIGURE 38: EXAMPLE OF PRESSURE AMPLITUDE AND PHASE INFORMATION OF THE MEASURED AND PROJECTED PLANES. PICTURES A1 AND A2 SHOW THE AMPLITUDE AND PHASE INFORMATION OF THE PLANE MEASURED IN FRONT OF THE TRANSDUCER AT 50 MM AWAY FROM THE SURFACE. PICTURES B1 AND B2 SHOW THE AMPLITUDE AND PHASE INFORMATION OF THE PLANE MEASURED IN FRONT OF THE TRANSDUCER AT 100 MM AWAY FROM THE SURFACE. PICTURES C1 AND C2 SHOW THE RESULTS OF PROJECTING A1 AND A2 TO 100 MM AWAY FROM THE SURFACE. THEY ARE ALMOST IDENTICAL TO THE MEASURED PLANES B1 AND B2.

In these figures, one of the transducer's elements were randomly chosen to show the performance of the element by using the projection algorithm. To make it more clear, when the hydrophone measures the plane in front of the transducer for each element at specific steps ( $1 \mathrm{~mm}$ here), the resulting measured signals would give us a 3D matrix. The first 2D is related to the position of the hydrophone. This position would be moved by the size of the step $(1 \mathrm{~mm})$ to create the plane $(60 \mathrm{~mm} \times 60 \mathrm{~mm})$ in front of the transducer. Fourier transform related to this position called spatial Fourier transform. The $3^{\text {rd }}$ dimension of the matrix is the time period of the measured signals. The Fourier transform of this dimension called temporal Fourier Transform.

Figure 38a1 shows the amplitude of a measured plane in water at temporal frequency of $0.5 \mathrm{MHz}$ (applying the Fourier transform on the $3^{\text {rd }}$ dimension of the matrix and picking up the plane for $0.5 \mathrm{MHz}$ ) at $50 \mathrm{~mm}$ in front of the transducer. Figure $38 \mathrm{a} 2$ shows the phase information of the same plane. These two planes then were projected to 
$100 \mathrm{~mm}$ in front of the transducer resulting in Figures $38 \mathrm{~b} 1$ and $38 \mathrm{~b} 2$. This was done by 1) taking the 2D spatial Fourier transform of the plane (the first two dimensions of the matrix) and then 2) applying equation (5) and lastly, 3) taking the 2D inverse spatial Fourier transform. Finally, a plane was measured with the same temporal frequency at $100 \mathrm{~mm}$ in front of the same transducer and the same element. The results of temporal Fourier transform ( $3^{\text {rd }}$ dimension) at $0.5 \mathrm{MHz}$ frequency are presented in Figures $38 \mathrm{c} 1$ and $38 \mathrm{c} 2$. By comparing Figures $38 \mathrm{~b} 1$ and $38 \mathrm{~b} 2$ with Figures $38 \mathrm{c} 1$ and $38 \mathrm{c} 2$, the capability of the algorithm in determining phase and amplitude information at any distance in front of the transducer can be seen. The same procedure was done to get the plane information at the surface of transducer by back-projecting the measured plane for some of the elements.

The plane measurements was taken for some random elements for evaluating the results. In order to save time, a line rather than a plane was measured for the rest of the elements. Principles of line measurements are exactly the same as procedure applied for plane measurement. In the line measurement, there is one dimension of a line which was created by the moving steps of the hydrophone in front of the transducer. The second dimension is the measured time of the signal, resulting in a two dimensional matrix of the recorded signals. The rest of the procedure for back-projecting the line to the surface of the transducer is exactly as same as the one explained for the planes.

\subsection{Designing an Expander}

The data acquisition system is capable of sending/receiving 128 pulses at the same time. In addition, it is capable of controlling over the channels, defining filters for them, and amplifying the received signals. Most of the hardware parts are from National 
Instrument (NI®). Diagnostic Sonar is the company which assembled these parts and defined software for the parts and introduced FI Toolbox Toolbox ${ }^{\circledR}$ [184]. FI Toolbox, therefore, consists of a range of hardware and software modules for ultrasonic data acquisition and imaging.

Most of the hardware are based on the FlexRIO® custom instruments and processing [185]. The list of the hardware can be found at [184]. The main processor of the system is PXIe-8135 which is Core i7 embedded controller for PXI Express systems, 2.3 GHz Quad-Core PXI Controller. It is mostly used for processor-intensive, modular instrumentation, and data acquisition applications.

One of the challenges in designing the imaging system was connecting the transducers to the control and data acquisition system. In the first design, coaxial cables were used. Such coaxial cables could be connected to array interface of the acquisition system on one side and to connectors of the ribbon cables on the other side to be later connected to the transducers. Figure 39 shows the array interface (DSL-FIZ) of the data acquisition system and the design of coaxial cabled for connecting the transducers to the channels of the array interface. 

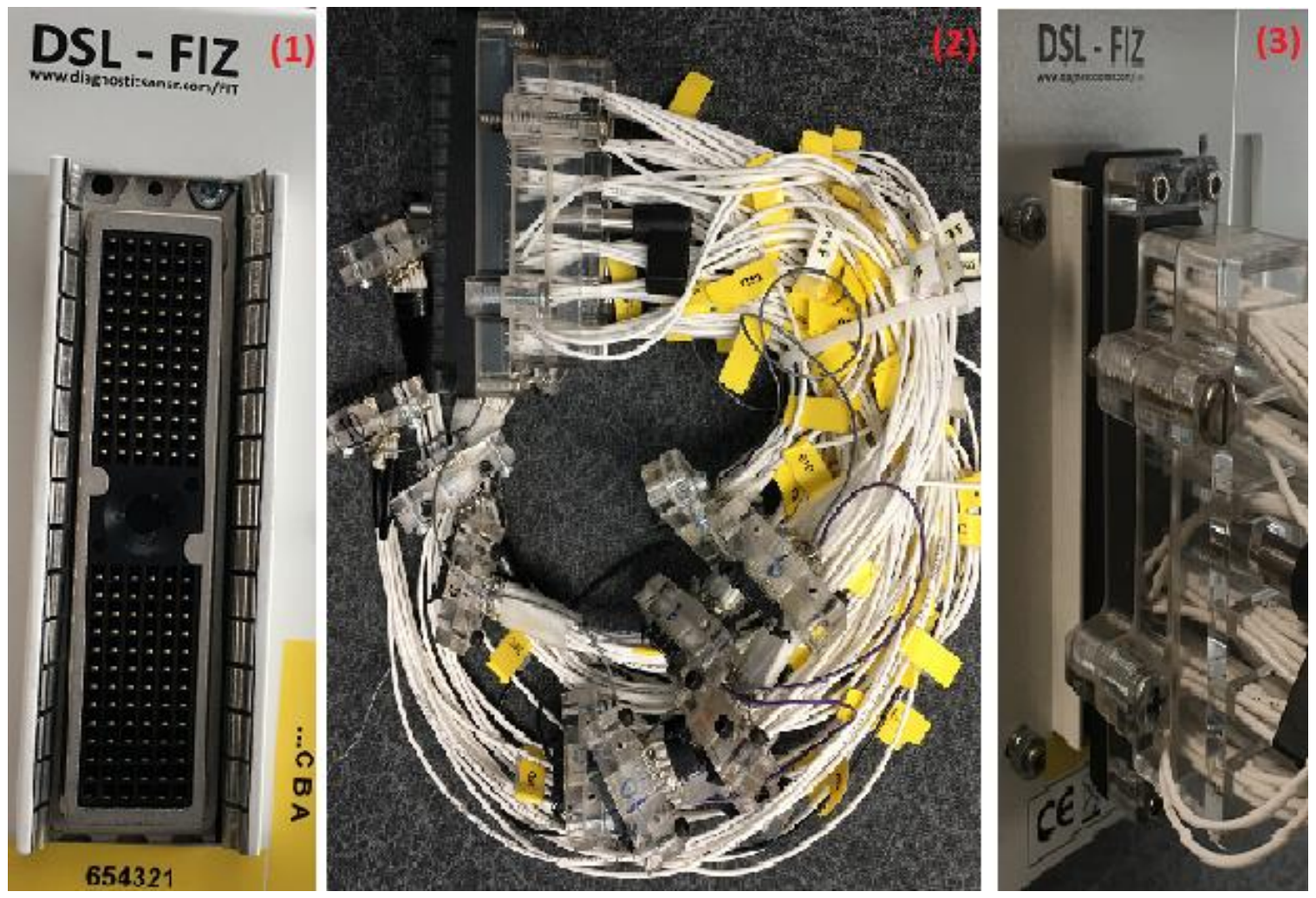

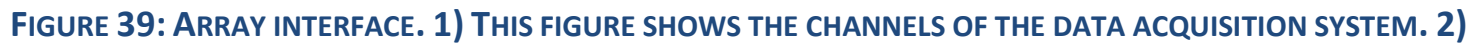
COAXIAL CABLES DESIGNED FOR CONNECTING THE CHANNELS OF THE ARRAY INTERFACE TO THE RIBBON CABLES OF THE TRANSDUCERS. 3) CONNECTING OF THE COAXIAL CABLES TO THE CHANNELS OF THE DATA ACQUISITION SYSTEM.

This picture shows the initial design of connecting coaxial cables to the array interface of the control and data acquisition system. These coaxial cables would be connected to the ribbon cables of the transducers from the other ends. The number of the cables (128) and tracking the connectivity of each of them make the design impractical and, therefore, a PCB was designed to provide easier and safer connections for the transducers (Figure 36).

In order to make the design more robust and practical, two PCBs were designed which would be connected to the channels by mounting on special connectors from DL series of Cannon ZIF connectors. The PCBs designed in a way that they mounted on the ZIF connectors and provide electrical connections between the channels and connectors 
mounted on the surface of the PCBs (Figure 36). Twelve number of connectors are mounted on the PCBs (6 on each). Connectors can be connected to the ribbon cables providing electrical connections for either 10 elements of transducers (Top position, Figure 33) or 9 elements of transducers (bottom position, Figure 33). Ribbon cables provide electrical connectivity to the multiplexers on one side and to the transducers on the other side. Every other wires in the ribbon cables are grounded for elimination of the noise. In addition, two middle layers of each PCB are also grounded (results of signal to noise ratio and crosstalk are explained in Chapter 5).

The imaging system is composed of around 500 sending and receiving elements. But, the data control and acquisition system supports up to 128 elements. Therefore, an expander is needed to cover more number of elements of about 500. The strength of the received signals are around $1 \%$ of the transmitted signals after passing the human skull. Therefore, the expander should be designed in a way that it sends high voltage generated signal from the data and control acquisition system. It should also have minimum crosstalk between the channels as it could damage the received signal.

The expander was designed using multiplexers. The multiplexers are dual 4channel analog multiplexers capable to switch up to $72 \mathrm{v}( \pm 36)$. The multiplexer is designed by Maxim with the part number of MAX14753 (Appendix C). Functional diagram of the MUX is shown in Figure 40. 


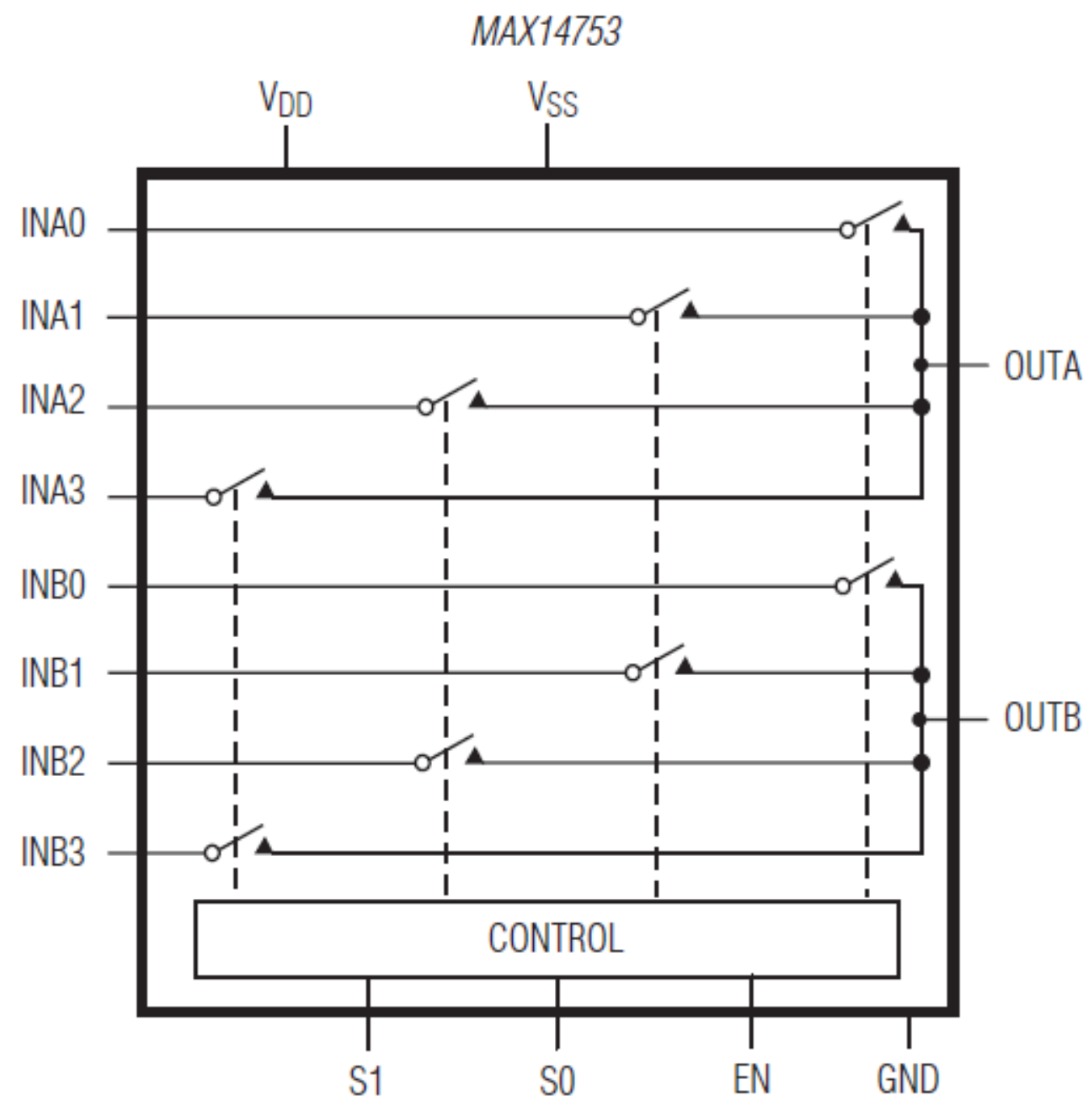

Figure 40: Functional diagram of the MUX.

The VDD supply voltage range is between +10 and +36 . These numbers are -10 and -36 for the VSS. The analog signal range for the MUX is between VSS (-36) and VDD (+36). When the IC is working, the maximum supply current is $600 \mu \mathrm{A}$ for each VDD and VSS channels. Typical transition time between the channels is $10 \mu \mathrm{s}$. Typical value for the voltage of the crosstalk is $62 \mathrm{~dB}$. Figure 41 shows the values of crosstalk based on the frequency (see Chapter 5 for more details). Other important features of the multiplexer could be found at Appendix C. 


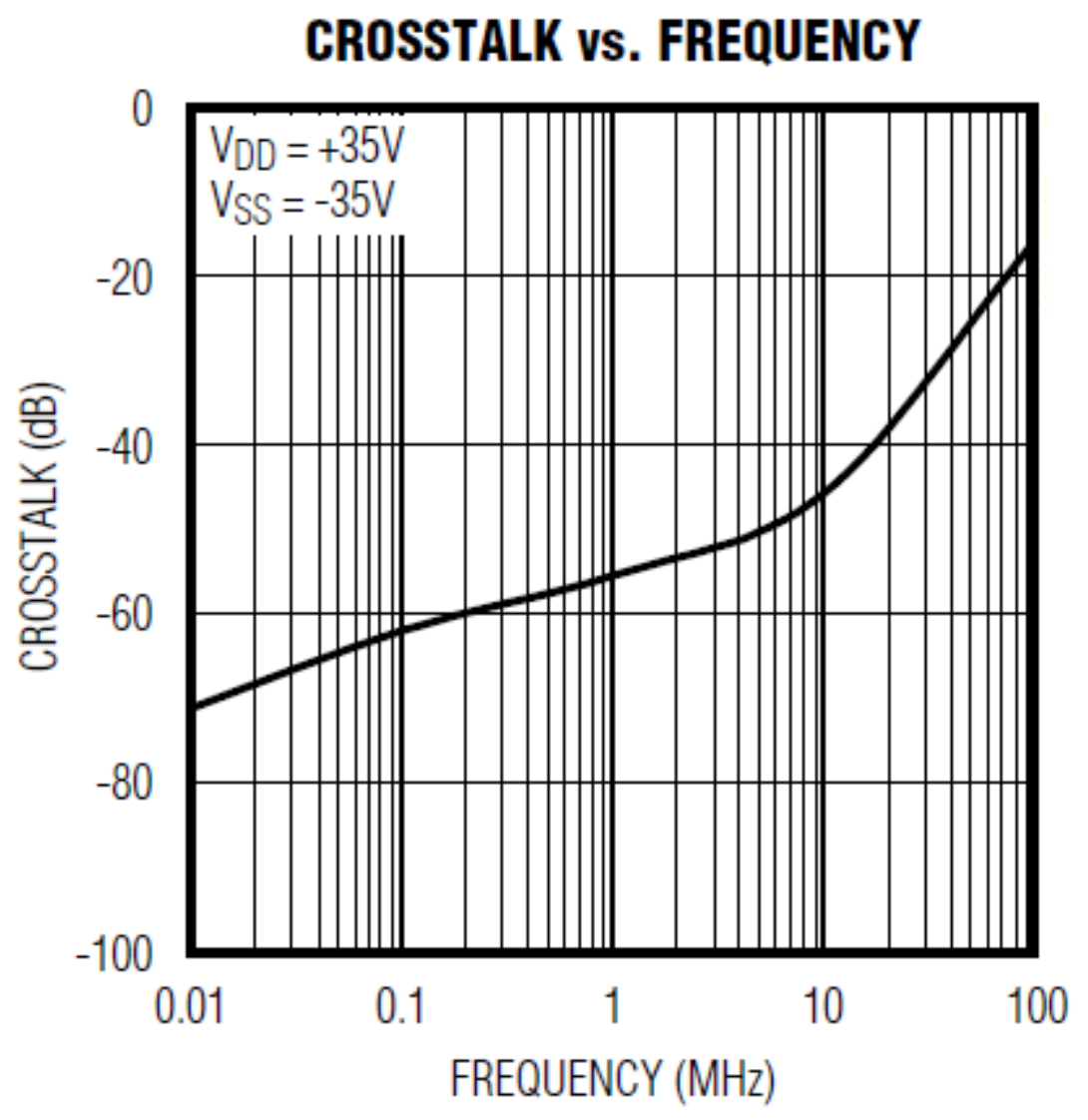

Figure 41: Crosstalk VS Frequency for the MUX.

Each of the 4 channel multiplexers were connected to 4 elements and the output of the multiplexer connected to the control and acquisition system. Therefore, each channel of the system would support up to 4 transducer elements and the total of 512 elements (Figure 42). 


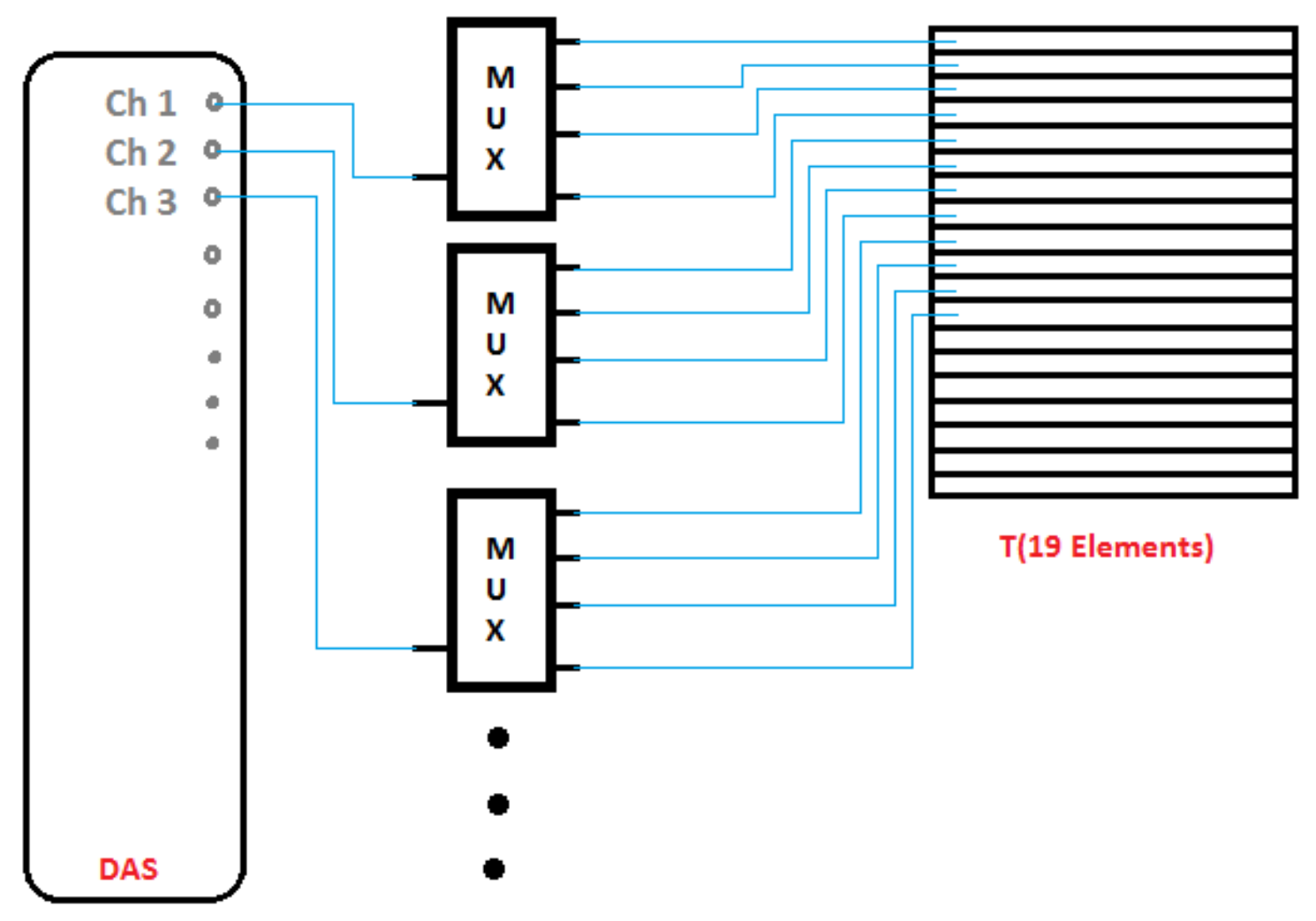

FIGURE 42: DESIGN OF THE EXPANDER. THIS PICTURE SHOWS ONE OF THE TRANSDUCERS (T) CONNECTED TO THE MULTIPLEXERS (MUX). THE OUTPUTS OF THE MUXS ARE ALSO CONNECTED TO THE DATA ACQUISITION SYSTEM (DAS).

In this picture, DAS shows the control and data acquisition system. Three channels of the system are connected to the inputs of the bidirectional MUXs (multiplexers). The MUXs, on the other side, are connected to the elements of the transducers $(\mathrm{T})$. By such a design, each channel of the system would be responsible for 4 elements of the transducers. As DAS has 128 number of channels, number to 512 channels could be increased by using 128 MUXs. But, as each transducer has 19 elements, 114 channels of the control and data acquisition system were used and multiplexed each channel to support 456 number of elements. 
The final PCB design for the expander is a 4 layer PCB. The design is done by using Eagle ${ }^{\circledR}$ PCB design software (Figure 43). Auto routing is used for the first and the last layers for traces between the components.

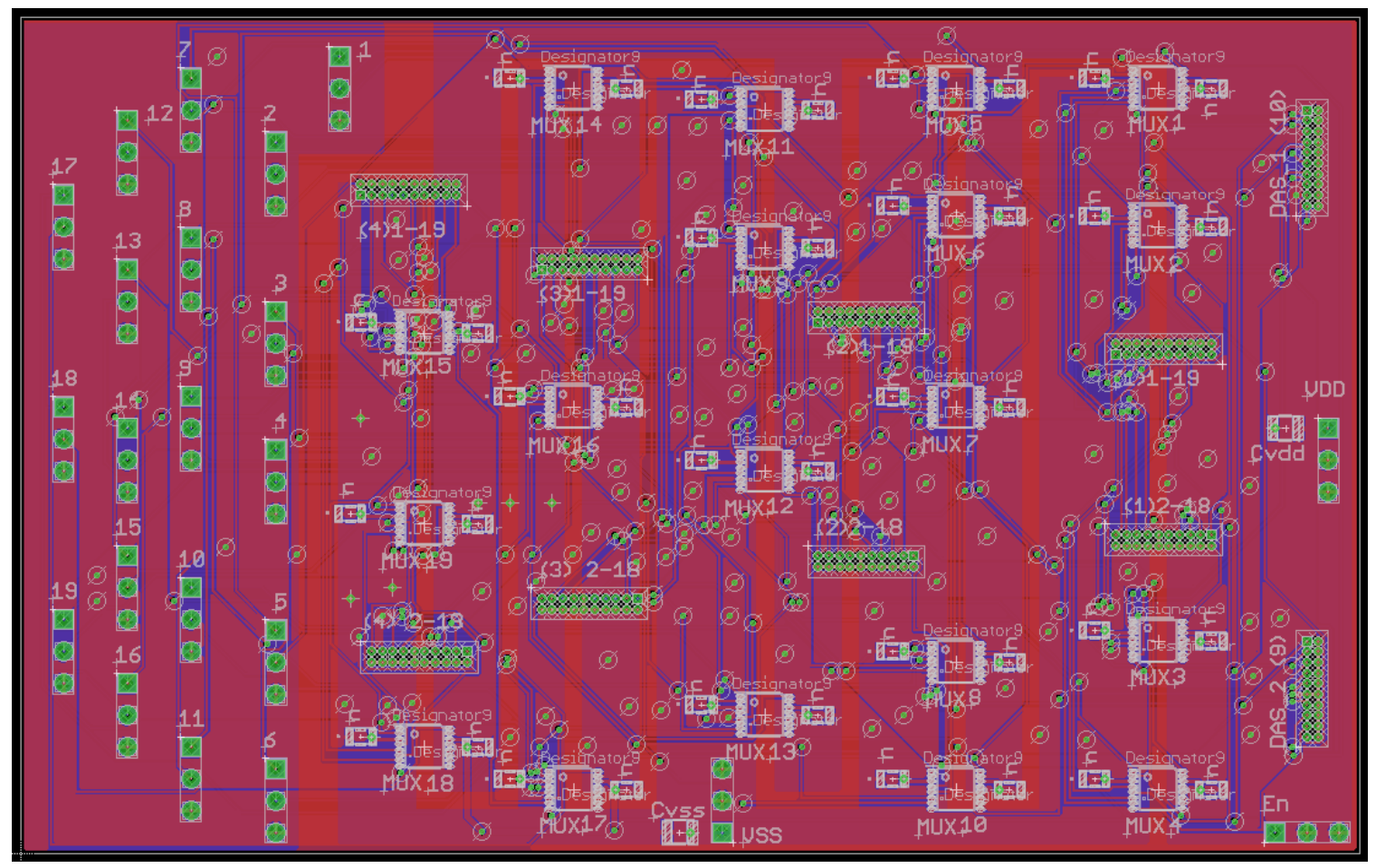

FIGURE 43: TOP VIEW OF THE PCB VIEW.

The first inner layer has 3 layers of copper (second layer). One plane of copper which was connected to VDD (maximum of $+36 \mathrm{~V}$ ). The second plane of copper was connected to VSS (Minimum of $-36 \mathrm{~V}$ ). These two planes are designed to be on the second layer for two reasons. First, most of the AC traces are in the top layer and therefore, by putting VDD and VSS layers on the second layer, the noise would be reduced for the AC traces. Second, VSS and VDD should provide voltage and current for 114 number of multiplexers on each PCB. Therefore, having a plane of copper for each of the VSS and VDD can decrease the resistance and therefore, less voltage reduction. The maximum required current for each multiplexer is $600 \mu \mathrm{A}$ (Appendix $\mathrm{C}$ ). As there 
are 114 multiplexer in total, the DC power supply should be capable to provide less than around $70 \mathrm{~mA}$ with typical current of around $30.78 \mathrm{~mA}$. The third copper layer of the second PCB layer is ground which covers the DC control pins of the multiplexers (Figure 44).

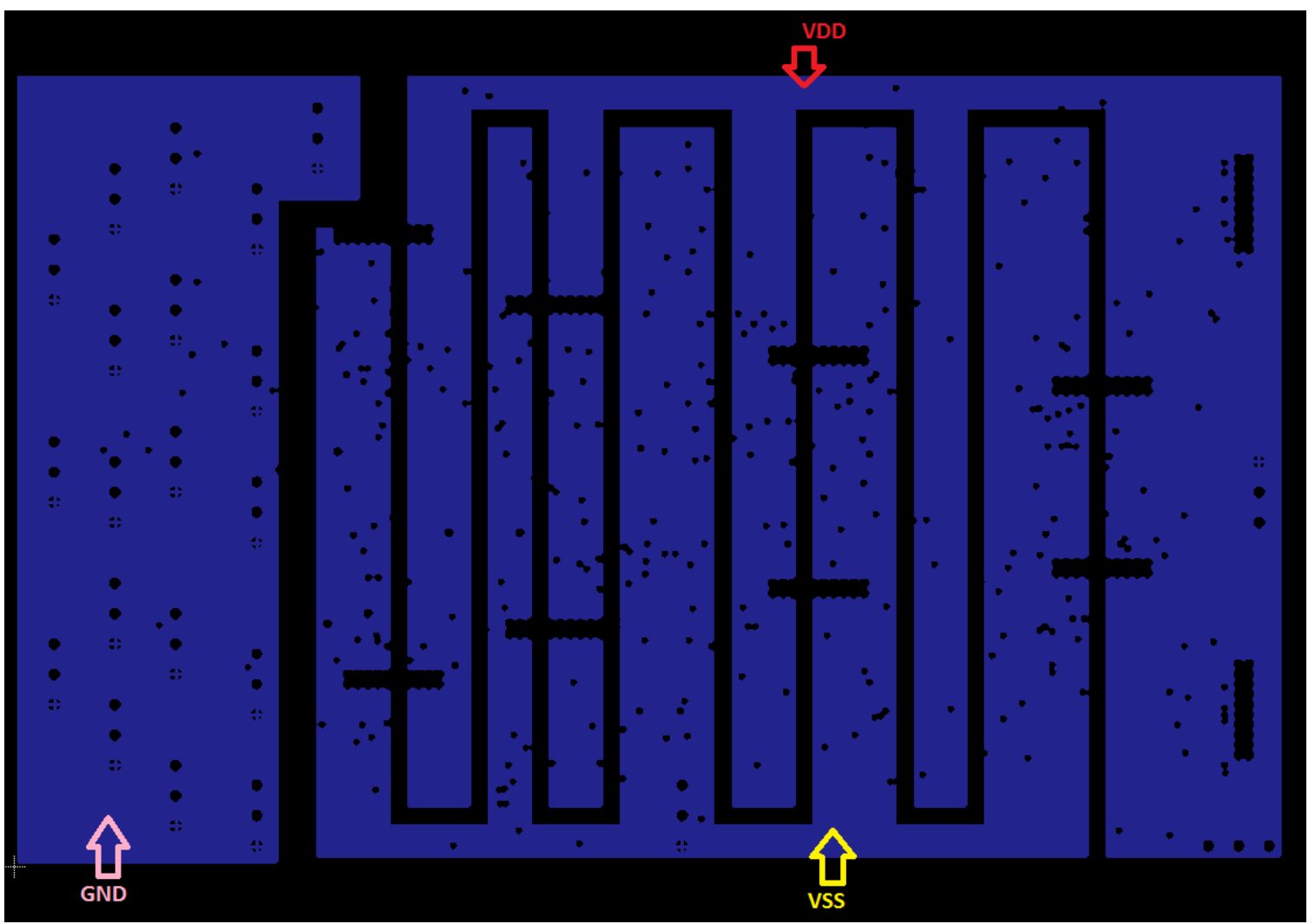

FiguRE 44: SECOND LAYER OF THE PCB.

The second layer designed in a way that the multiplexers are mounted between the VSS plane and the VDD plane. The second inner layer of the PCB is ground $\left(3^{\text {rd }}\right.$ layer). Figure 45 shows this layer. 


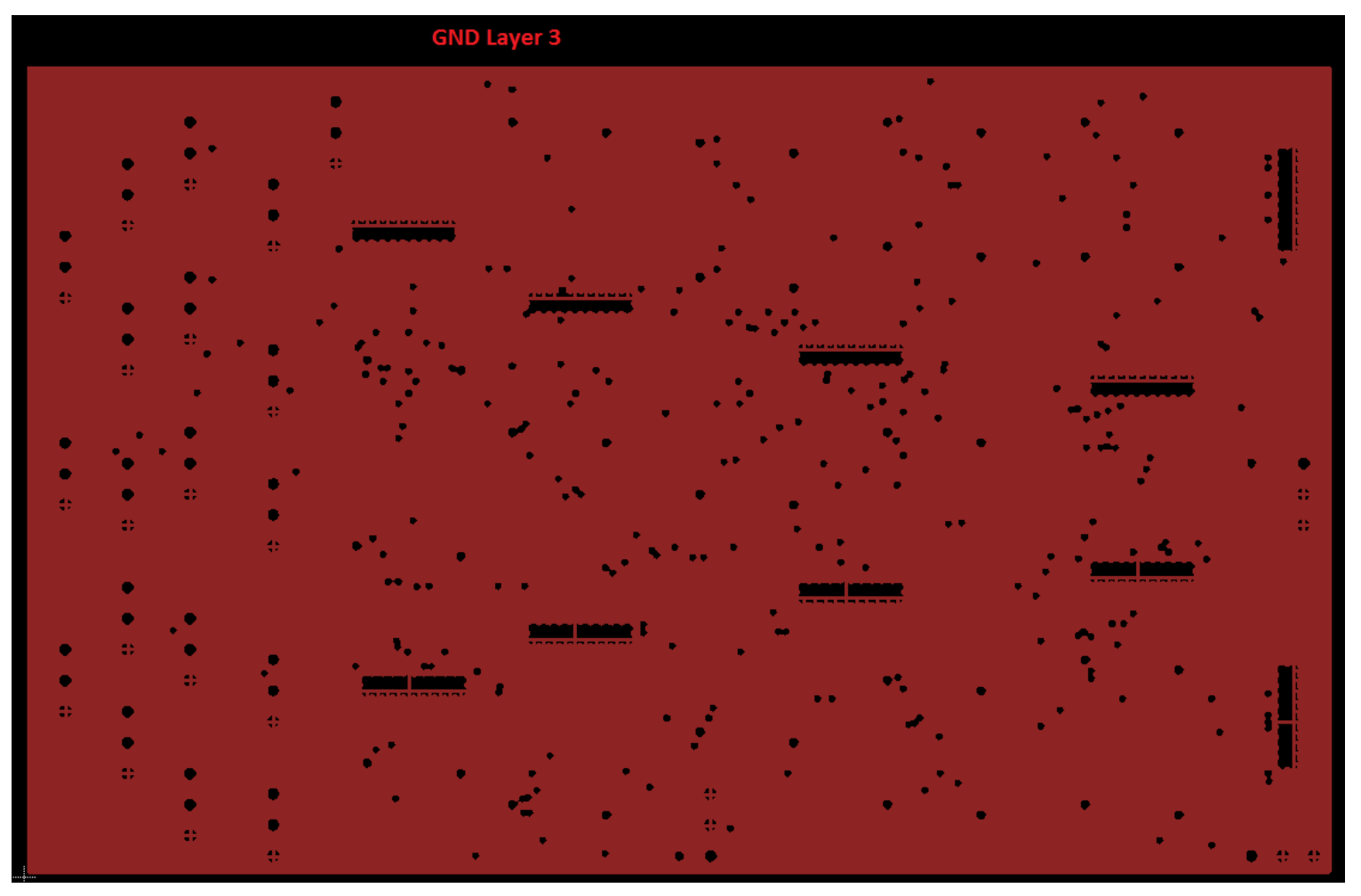

FIGURE 45: 3RD LAYER OF PCB.

The reason of considering the $3^{\text {rd }}$ layer as ground is that the DC traces are in the last layer of the PCB and, therefore, the ground layer would eliminate the noise. The traces of the last layer (bottom layer) is shown in Figure 46. 


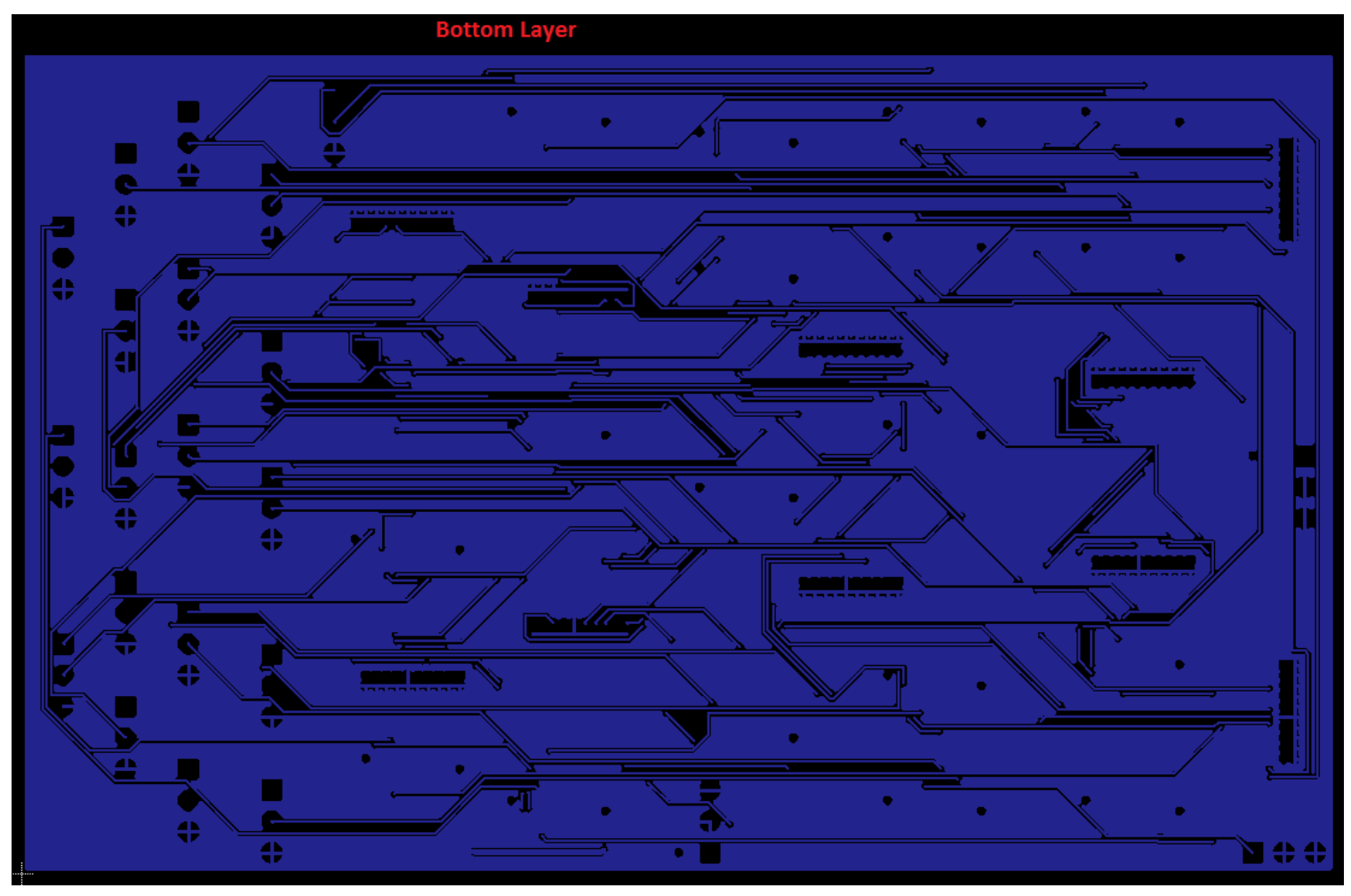

FIGURE 46: BOTTOM LAYER OF PCB.

As it is shown in Figure 47, each PCB has 19 multiplexers (MUX). For each MUX, inputs are connected to 4 elements of each transducer on one side. The output is connected to one channel of data acquisition system. These connections from MUXs to the data acquisition system channels and the transducers are provided by the connectors which would be connected to the ribbon cables. As mentioned before, every other wires in the ribbon cables are grounded for noise elimination.

The PCBs were designed by eagle ${ }$ and were sent to PCBWay® [186] for fabrication and assembly of the components. One of the boards was first fabricated with assembled components to test. Each PCB covers 4 transducers (76 elements). As the total number of the transducers is 24, the total number of ordered PCBs is 6 to cover all transducers. 
Two $1 \mu \mathrm{F}$ ceramic capacitors are connected from VSS and VDD to ground to eliminate the potentially presented AC noise. In addition, for each MUX, two $0.1 \mu \mathrm{F}$ ceramic capacitors connected very close to VSS and VDD pins of the MUXs. Figure 47 shows one of the final assembled PCBs.

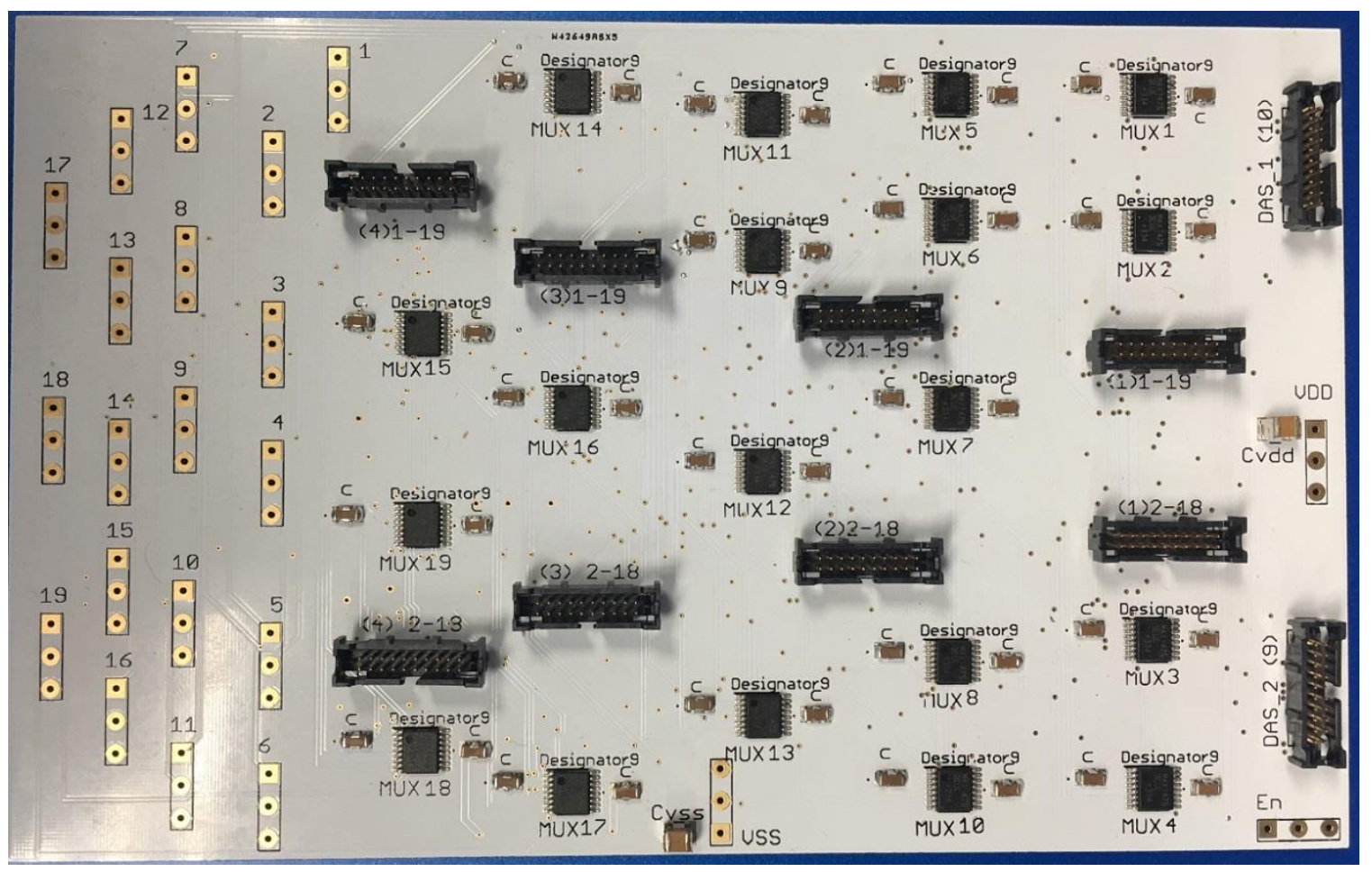

FigURE 47: FinAL ASSEMBLEd PCB WITH 19 MUXs ON IT. EACH PCB CONNECTS 76 ELEMENTS TO 19 CHANNELS OF THE DATA ACQUISITION SYSTEM.

The PCB was then tested to evaluate the functionality of all the MUXs as well as electrical crosstalk (see Chapter 5).

One of the shortcomings of the design is that whenever one of the transducer elements is firing the pulses, the three nearby elements which are connected to the same MUX cannot collect the received signals as they are not connected to the control and acquisition system (Figure 48). 


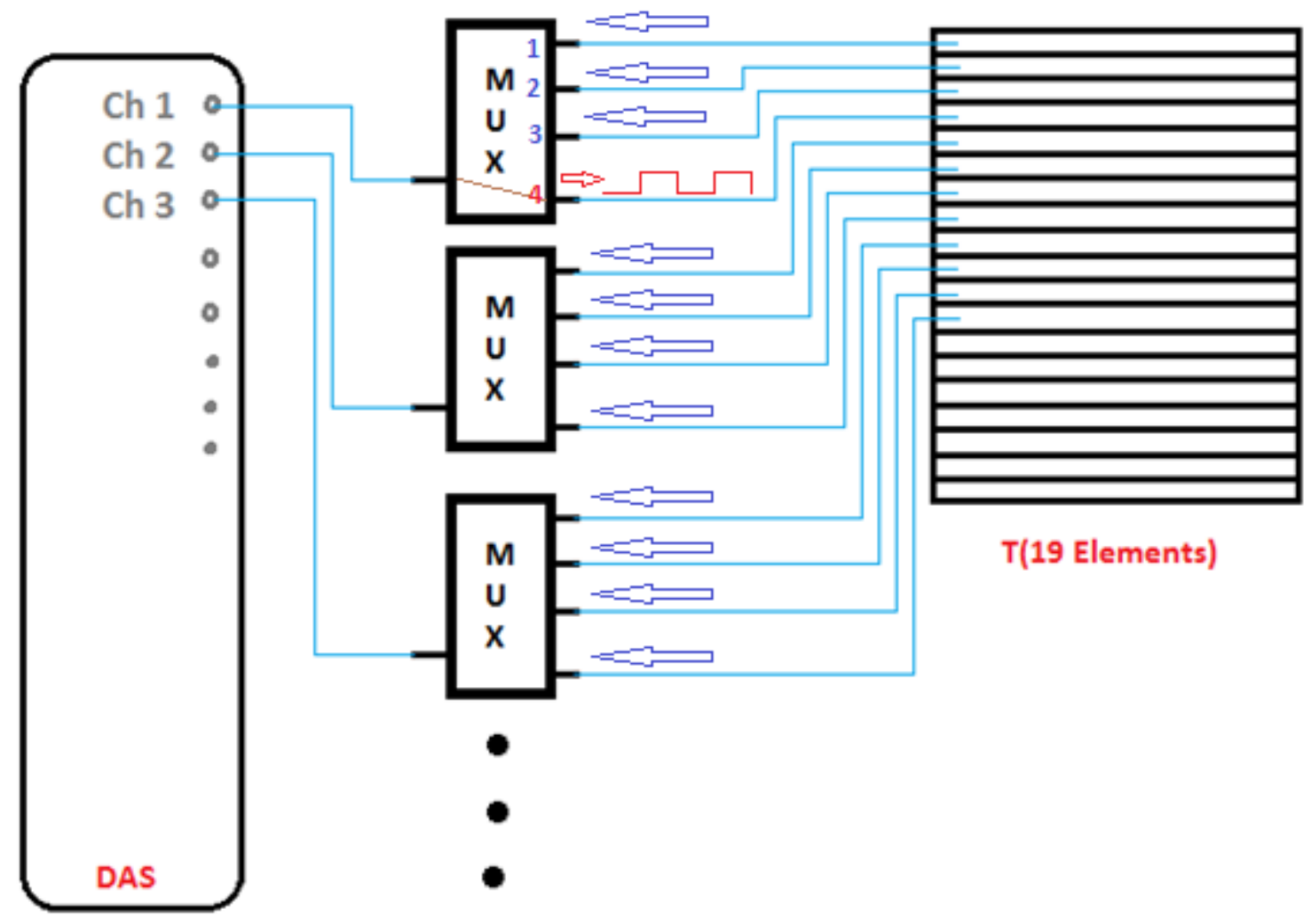

FIGURE 48: EXPANDER. SHORTCOMING IN DETECTION OF THE RECEIVED SIGNAL FROM ALL THE ELEMENTS.

Figure 48 shows an example of how the received signals are collected in full matrix capture (FMC). In this example, channel 4 of the first MUX is firing a signal (defined by data acquisition system (DAS)) and all other elements are collecting the received signals. Switching between the channels of the MUXs are controlled by using Arduino Due (Figure 49). When channel 4 of the first MUX is firing signal, the received signals from all other elements would be collected by switching between the channels of other MUXs. Therefore, by 4 times switching between the rests of the MUXs, all the received signals from the rest of the MUXs can be collected. However, the three neighbor elements to the firing element are not connected to any channels of the DAS (channels 1 , 2, and 3 in the first MUX (in blue color) of Figure 50). Therefore, every time by 
collecting the signals from the elements, 3 received signals would be lost. For example, if we have 512 number of elements, we will be able to collect 509 received signals.

In order to solve the problem of 3 missing signals, the DAS should be synced to the Arduinos (which controls the MUXs, Figure 49). The control and data acquisition system is capable of defining the pulse repetition frequency (PRF) from $100 \mathrm{~Hz}$ to around $2 \mathrm{KHz}$. A range in which the received signal would be collected can also be defined by DAS. So, the minimum time of firing signal is every $10 \mathrm{~ms}$ up to the maximum of $0.5 \mathrm{~ms}$. But, the lower the PRF, the less chance of detecting the signal in the defined range by DAS. The maximum range which can be defined by the system is around $200 \mu \mathrm{s}$.

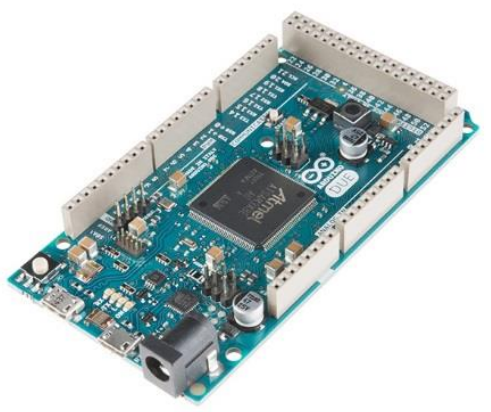

Figure 49: ARDUINO DUE.

The Arduino Due is based on a powerful 32bit CortexM3 ARM microcontroller made programmable through Arduino IDE. It has 54 digital input/output pins, 12 analog inputs, 4 UARTs (hardware serial ports), an 84 MHz clock, a USB-OTG capable connection, 2 digital to analog (DAC), 2 TWI, a power jack, an SPI header, a JTAG header, a reset button and an erase button. Arduino Due runs at $3.3 \mathrm{~V}$ and the maximum voltage that the $\mathrm{I} / \mathrm{O}$ pins can tolerate is $3.3 \mathrm{~V}$. The reason of choosing this type of Arduino is its capability in processing at high speed. 
The Arduinos are then synced to the DAS and therefore, by switching the channel of the firing MUX by reasonable delay after the signal is fired, the received signals from the other channels are collected. In other words, in order to have the three missing signals, firing channels of the MUXs should be switched to receiving from the three missing channels. In this way, all the signals from those missing channels are collected. These switching should be done after the sending signal is passed. So, a range of $200 \mu \mathrm{s}$ is available to collect the received signals (Figure 50).

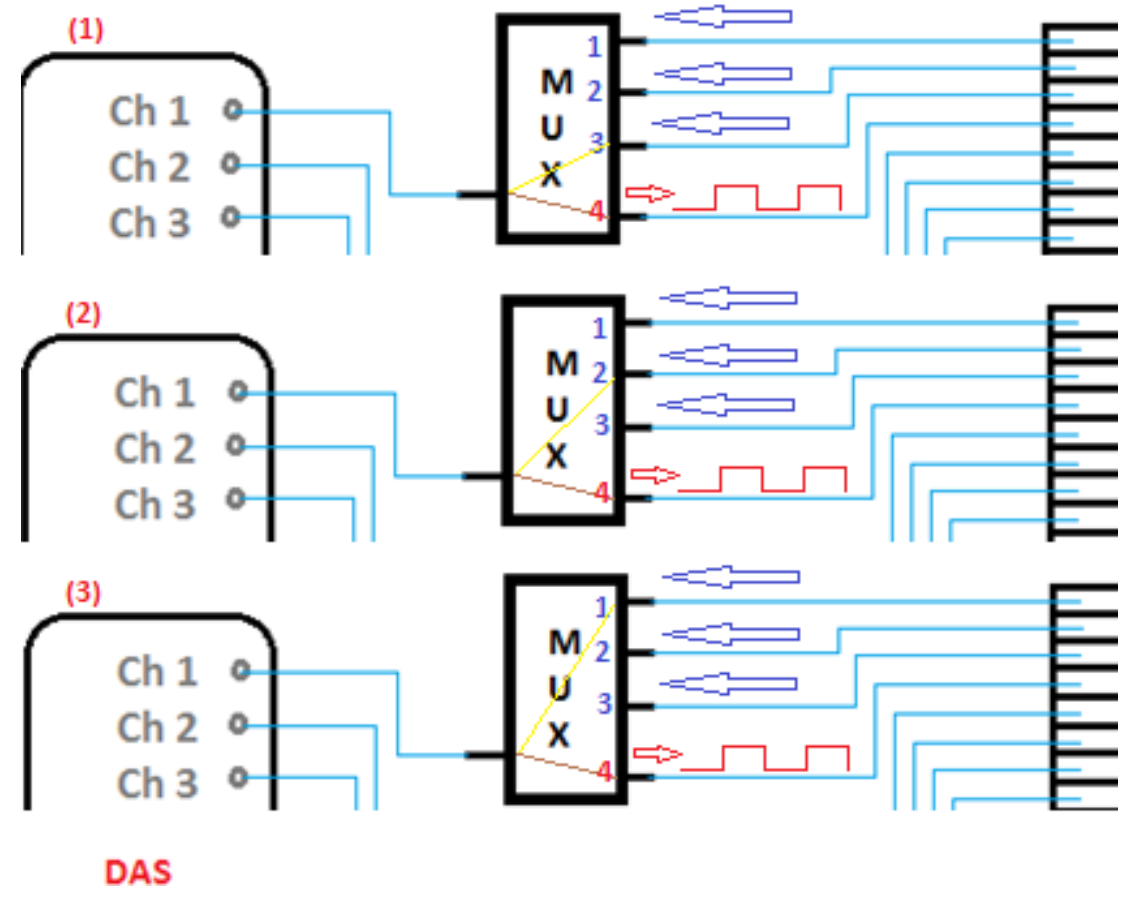

FIGURE 50: SWITCH BETWEEN THE CHANNELS OF THE MUX IN WHICH THE SIGNAL IS FIRING.

As the maximum tolerated voltage of the $\mathrm{I} / \mathrm{O}$ pins of the Arduinos is $3.3 \mathrm{~V}$, simple voltage divider was used to decrease the firing voltage of the DAS to less than $3.3 \mathrm{~V}$. By using a potentiometer, the voltage for Arduino input can be easily adjusted. The defined voltage of the DAS has minus peak which is not readable for the Arduino. In order to 
have only positive pulses, a simple diode was used to ground the negative portion of the pulses (Figure 51).

\section{Vpp from DAS channel}

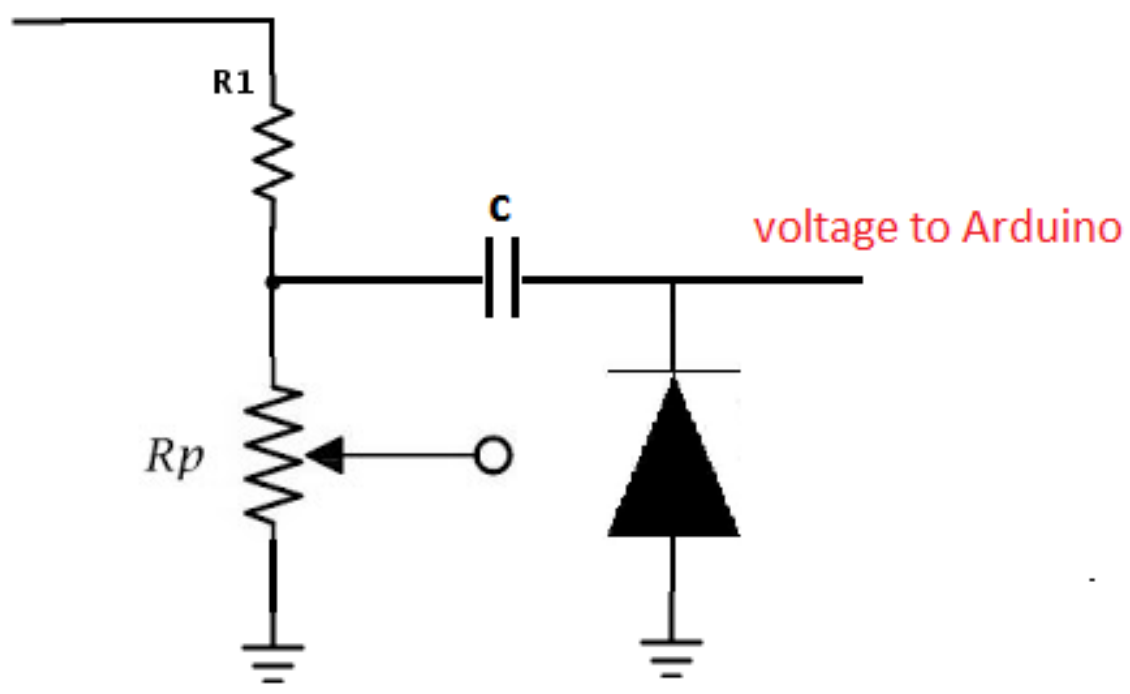

FiguRE 51: CLAMPER OR DC RESTORATION CiRCUIT.

The output of the circuit in Figure 51 can also be used as an input of an XOR (Exclusive or) with the other input of the ground. Therefore, when the output of the circuit is positive (or 1), the output of the XOR would be on (or 1) and can be used for switching between the channels of the MUXs. In the current design, the Arduinos were used to control the switching as we already should use 4 of them to control all the MUXs. 


\section{CHAPTER V}

\section{RESULTS}

In this chapter, the transducers functionality and the mechanical isolation of their elements are evaluated in Section 5.1. Characterization of the expander is addressed in Section 5.2. This section explains in details, how the crosstalk and the noise are measured. Solutions for eliminating the noise are addressed as well. Finally, the way of collecting data for image reconstruction using the expander is explained at the end of the section.

\subsection{Transducer Functionality}

In order to check the functionality of the transducers, the measured pressure field in front of the test elements was back-projected to the surface of the transducer for each element by using equation 10 (see Chapter 4, Section 4.3). Figure 25 shows the pressure amplitude in two of the back-projected planes. These planes show the Fourier transform 
of propagated plane at the surface of the transducer for two specific elements at temporal frequency of $0.5 \mathrm{M} \mathrm{Hz}$, which is the resonance frequency of transducers.
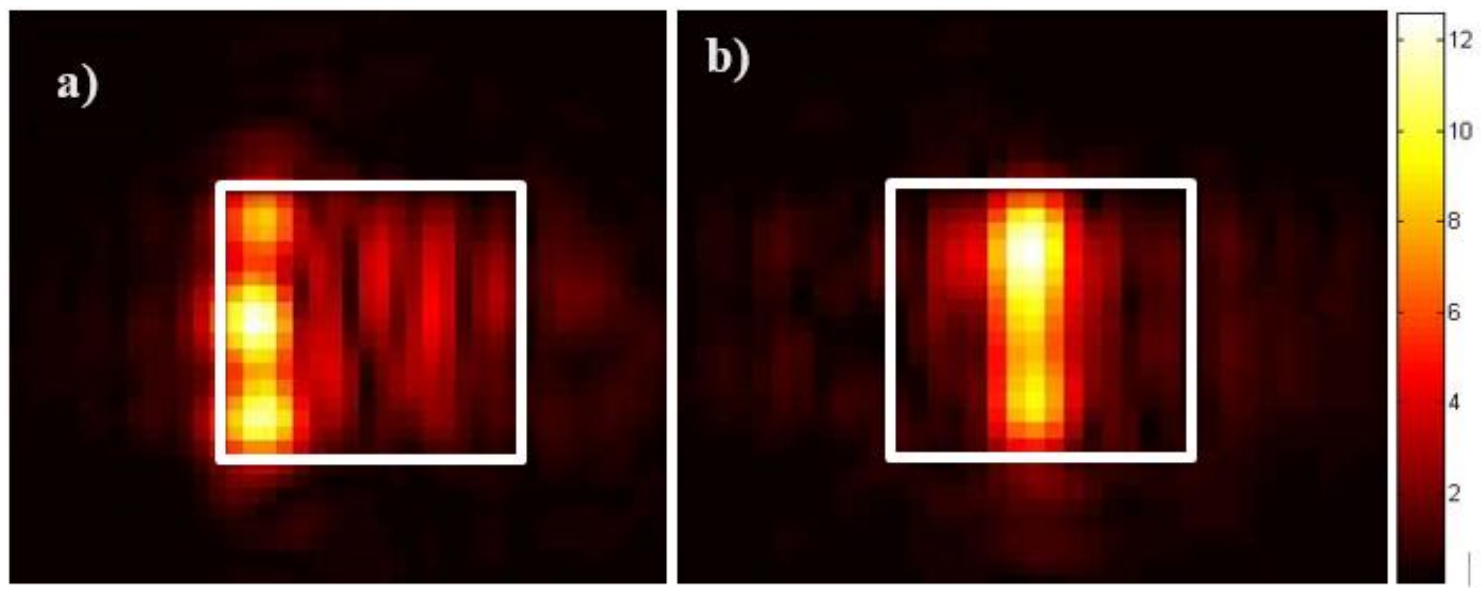

FigURE 52: PRESSURE AMPLITUdE OF TWO RANDOM ELEMENTS AT THE SURFACE OF THE TRANSDUCER.

Figure 52a shows pressure amplitude of the back-projected plane for an element located at the edge of transducer. It is obvious that the main pressure amplitude is caused by the element. Other elements have very minor impact on the plane. The same trend can be seen for Figure 52b, in which the propagated plane is for an element in the middle of the transducer. It can be seen that the $1 \mathrm{~mm}$ depth of the cuts is enough to separate the functioning of each element from the rest. Such vibration isolation of the elements is important as the final goal is to apply a new technique of diffraction tomography using these elements [1]. In diffraction tomography, the object of interest is surrounded by several elements of transducers which can send and receive ultrasound energy. When one of the transducers send the ultrasound energy, all of them would work as receivers and collect this energy. The procedure is followed by sending the energy from the second transducer and collecting again by all of them. Such a trend is continued until all the transducers act as transmitters (see Chapter 3 for more details). 
In order to test the mechanical isolation of the elements, line measurements (instead of planes) were taken under the assumption of symmetry. The line measurements were then back-projected to the surface of transducer to see the results. Figure 53 shows the results of two back-projected line measurements to the surface of the transducers. Two elements at the very end side of a transducer were selected to show the shifting of amplitude more clear.

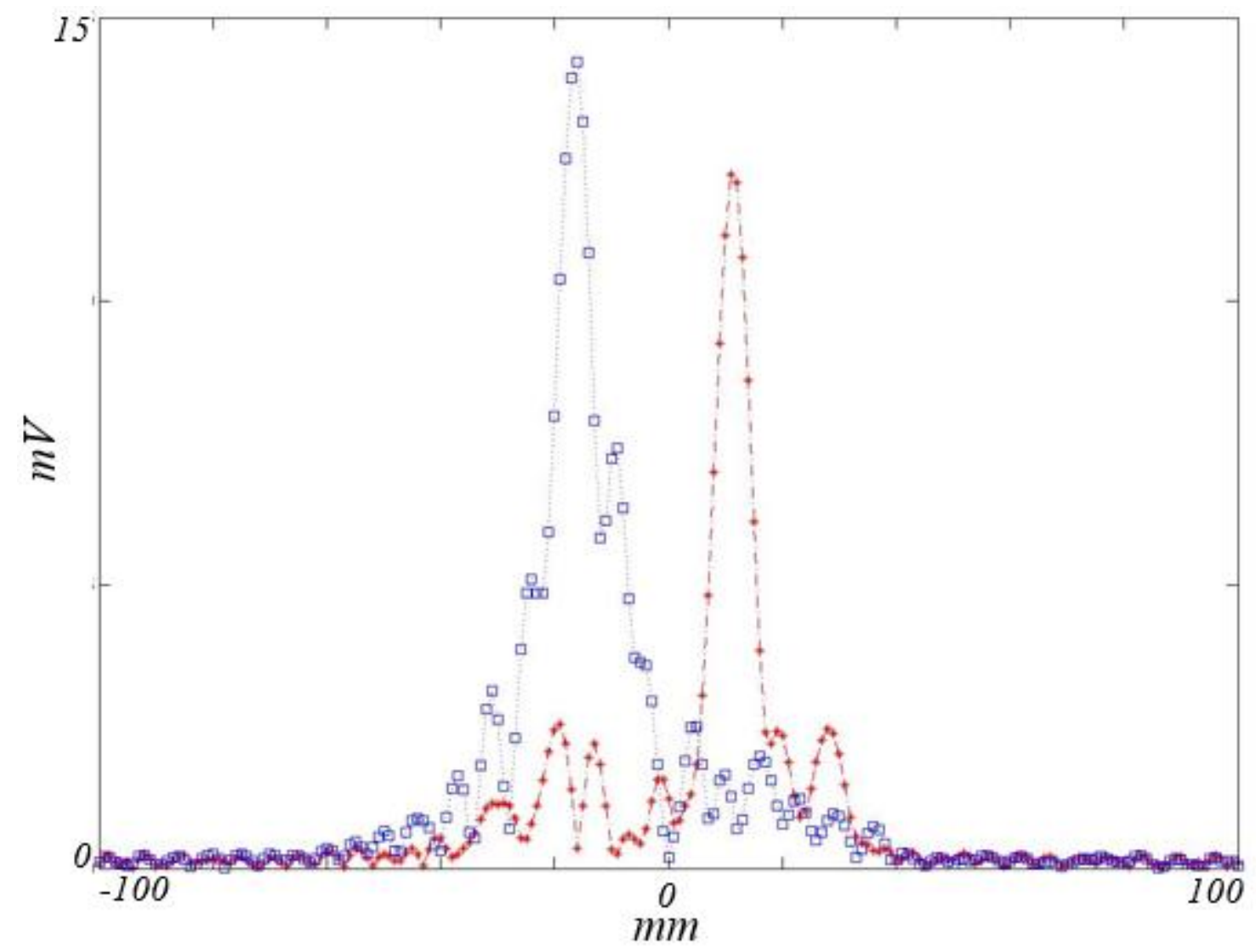

FIGURE 53: PRESSURE AMPLITUDE OF TWO ELEMENTS LOCATED AT VERY EDGES OF TRANSDUCER.

A line with a length of $200 \mathrm{~mm}$ perpendicular to the propagation axis at the distance of $50 \mathrm{~mm}$ in front of the transducer was measured. The results of back-projected line show the position of amplitude shifting by the activation of different elements from one side to the other side. Each amplitude in the picture represents one element on the 
surface of the transducer. For simplicity, we only show amplitude results from two elements at each side of a transducer. Therefore, the other amplitudes should be depicted between these twos with equal spacing from each other. Another important feature of the projected lines is that we did not have the decrease in amplitude more than $3 \mathrm{~dB}$, which is equal to $50 \%$ of the biggest amplitude.

\subsection{Expander Characteristics}

As explained in Chapter 4 (Section 4.4), the data and control acquisition system has 128 channels and, therefore, is capable of sending/receiving 128 signals at the same time. In order to provide electrical connectivity for all 24 fabricated transducers (456 elements) to the data acquisition system, six PCBs are designed to function as an expander between the transducers and the data acquisition system. Each PCB has 19 MUXs. Each channel of the data acquisition system could be connected to one MUX. The multiplexers are dual 4-channel bidirectional analog multiplexers capable to switch up to $72 \mathrm{v}( \pm 36)$ (APPENDIX C). Therefore, every four of the transducer elements are connected to the other side of the multiplexers (Figure 50). As each PCB has 19 MUXs, a total of 76 number of elements could be connected to each PCB. The total of 6 PCBs are designed to cover up to 456 elements of the transducer plates. Arduino Due® is used for controlling the switching between the channels of the MUXs. As explained in details in Chapter 4 , by 4 times switching between the channels, we can collect all the received signals for each elements. This requires fast switching of the Arduino for three nearby elements connected to the same MUX.

Each PCB was tested to evaluate the electrical isolation of all the channels for each MUX (electrical crosstalk). In addition, the ratio of signal to noise level was also 
evaluated. Figure 54 shows how the MUXs are connected to the data acquisition system on one side and to the transducer elements on the other side. Each board is connected to 19 channels of the data acquisition system. To test the functionality of the MUXs, ten channels (out of 19) are selected to be in the firing mode and the rest of 9 channels in the receiving mode. Therefore, the outputs of ten MUXs are connected to sending channels and the rest to the receiving channels. Then, every four pins of the sending MUXs were connected to voltage dividers and the outputs of the voltage dividers were connected to every four pins of the receiving channels (Figure 54). A PCB was designed with 4 different voltage divider (4 different attenuators) and the results of receiving signals and the crosstalk on other channels were evaluated on Oscilloscope. All the MUXs were controlled by an Arduino.

Three different square pulses ( 1 cycle with $20 \mathrm{Vpp}, 3$ cycles with $30 \mathrm{Vpp}, 3$ cycles with $40 \mathrm{Vpp}$ ) at frequency of $0.5 \mathrm{MHz}$, were sent from the data acquisition system to the sending MUXs and then to the attenuators. Figure 55 shows the user interface of the FI toolbox software in which the peak to peak voltage and the number of cycles for transmitting signals as well as the time gain control (TCG) waveform and the gain properties for the receiving signals could be modified. The picture shows an example of 2.5 cycles of transmitting signal with $40 \mathrm{Vpp}$ at frequency of $0.5 \mathrm{MHz}$. 

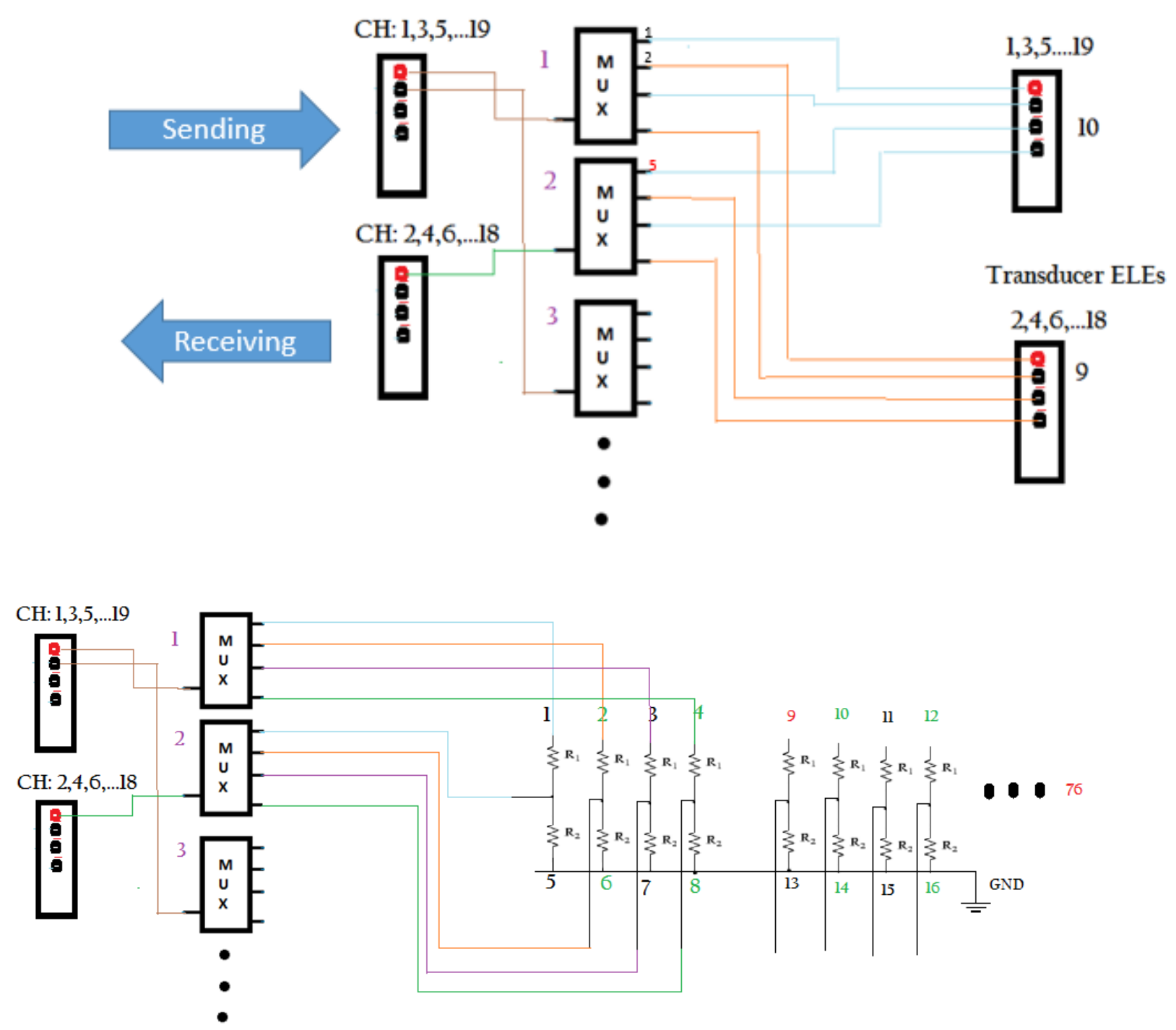

FigURE 54 : Testing PCB. ThIS FIGURE SHOWS HOW THE MUXS ARE CONNECTED tO the DATA ACQUISITION SYSTEM ON ONE SIDE AND TO THE TRANSDUCER ELEMENTS ON THE OTHER SIDE. IN ORDER TO TEST THE MUXS, EVERY FOUR CHANNELS OF THE SENDING MUXS ARE CONNECTED TO THE ATTENUATORS AND THE OUTPUT OF THE ATTENUATORS ARE CONNECTED TO THE RECEIVING MUXS. 


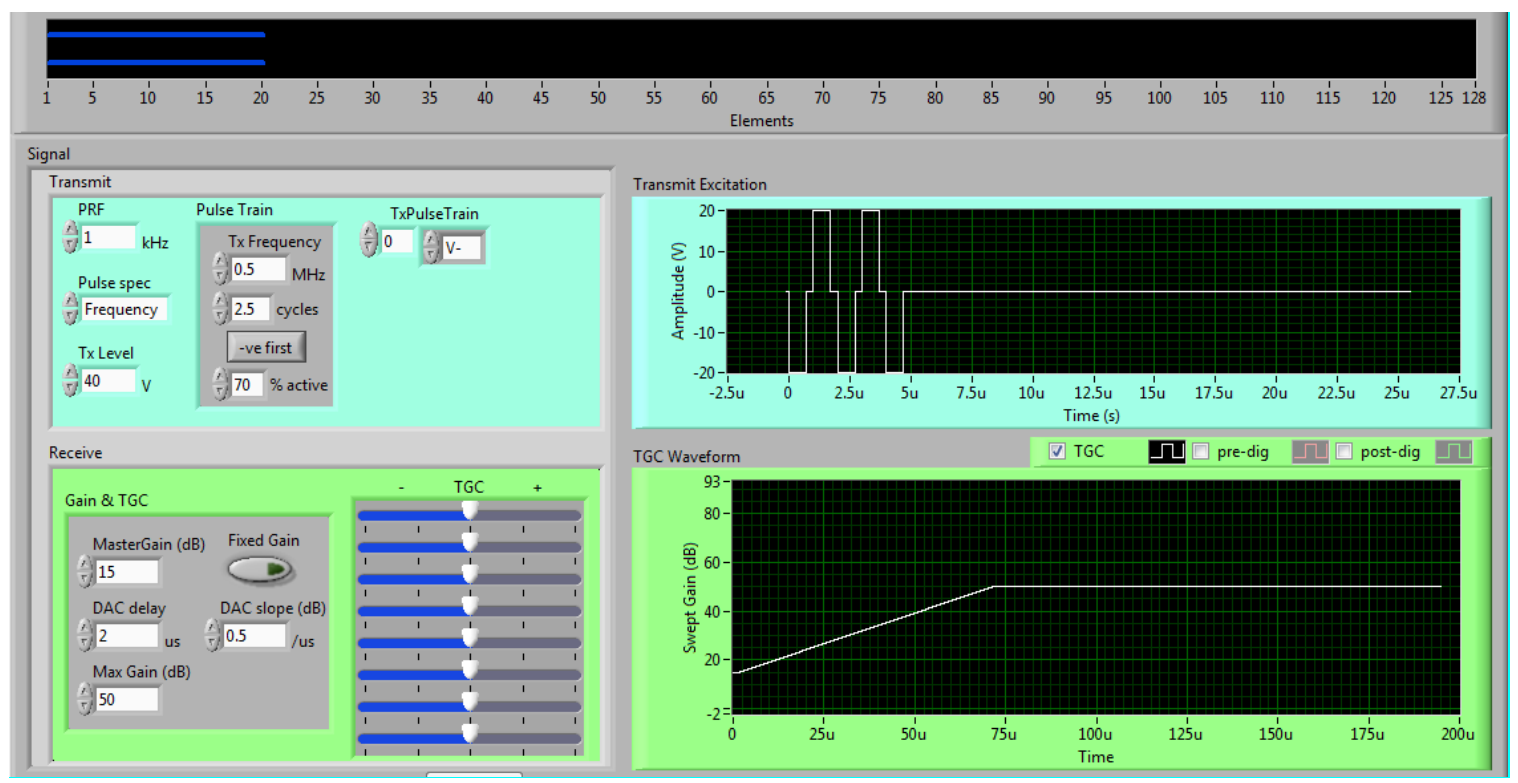

FIGURE 55: FI TOOLBOX SOFTWARE USER INTERFACE. IN THIS SECTION OF THE SOFTWARE, THE NUMBER OF TRANSMITTING CYCLES, PULSE REPETITION FREQUENCY, SIGNAL FREQUENCY, AND THE PEAK TO PEAK VOLTAGE OF THE SIGNALS FOR THE TRANSMITTING SIGNAL COULD BE DEFINED. THE RECEIVING GAIN AND THE TIME GAIN CONTROL (TGC) OF THE RECEIVING SIGNALS COULD ALSO BE DEFINED.

The same time gain control (TGC) was used for all the receiving channels throughout the experiments. Ribbon cables were shielded and the shields were grounded. At each time of sending the signals, only one of the input pins was connected to the output pin of the same MUX. By looking at the other pins, the crosstalk of around $-58.7 \pm$ $2.9 \mathrm{~dB}$ was detected. For calculating the crosstalk, the ratio of the root mean square voltage of the detected signals on not connected channels to the root mean square voltage values of the sending/receiving channels were calculated using equation (11).

$$
\text { Ratio }=20 \times \log _{10}\left(\frac{V n_{r m s}}{V s_{r m s}}\right)
$$

$V n_{r m s}$ is the root mean square voltage of detected noise and $V s_{r m s}$ is the root mean square voltage of transmitting/receiving signal.

The result of the ratios were close to the values reported on Figure 41. Figure 56 shows the typical detected signal on the channels of a MUX which are not connected to 
the input or output of the MUX. The connected channel is passing the square pulses (3 cycles with $45 \mathrm{Vpp}$ ) at the frequency of $0.5 \mathrm{MHz}$. It was then attenuated by about $-20 \mathrm{~dB}$ and connected to another receiving channel of the data and control acquisition system after passing the second MUX.
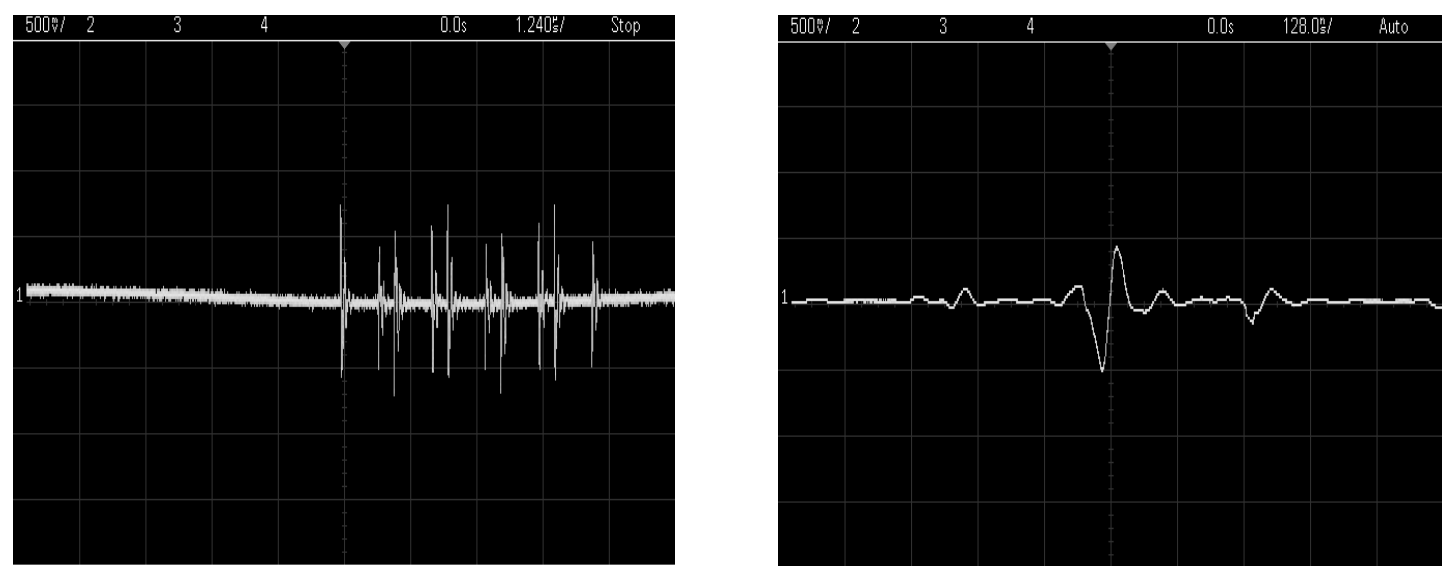

FiguRE 56: CROSSTALK. BOth IMAGES SHOW THE RESULT OF DETECTED SIGNAL AT A CHANNEL OF A MUX WHICH IS NOT CONNECTED TO THE OUTPUT/INPUT CHANNEL WHILE THE NEARBY CHANNEL IS PASSING THE TRANSMITTING SIGNAL OF ABOUT 40 VPP WITH THREE CYCLES AT FREQUENCY OF 500 KHZ. THE APPROXIMATE DETECTED CROSSTALK OF THE RECEIVED SIGNAL IS $\mathbf{- 5 8 . 7} \pm \mathbf{2 . 9}$ DB. THE SAME TREND WAS DETECTED FOR ALL THE TRANSDUCERS.

In Figure 56, the detected signal has the gain of about $-58 \mathrm{~dB}$ compared to the transmitted signal. The duration of the detected signal is about $128 \mathrm{~ns}$. Compared to the transmitted signal which has the duration of $2 \mu \mathrm{s}$, the rising and falling time of the detected signal is much faster.

The final design needs to cover around 500 elements; therefore, after testing the isolation and functioning of the MUXs, five more PCBs were ordered to be fabricated and assembled. By having six PCBs, the total of 456 elements could be connected to our 128 channels of the data acquisition system. 
Next, the transducers were tested before and after adding the expander to the system to evaluate the level of noise at each phase. For experiment setup, transducers were rigidly mounted on the wall of a water tank with propagation along the long axis of the tank. One of the elements of a transducer was selected to send 4 cycles of square pulses signal at $30 \mathrm{Vp}$-p withfrequency of 0.5 MHz. Then, a rectangular metal object was mounted on the way of axes of propagation in front of a transducer surface to reflect the ultrasound signal back to the transducer surface. The received signals from all the elements were collected by the control and data acquisition system: 1- directlty from the transducer and 2- after connecting the transducers to the expander. Actual signal is achieved by averaging the 50 measured received signals. Noise was extracted by subtracting the actual signal from the measured signals. Figure 57 shows the SNR histogram of the 50 measurements for each experiment.
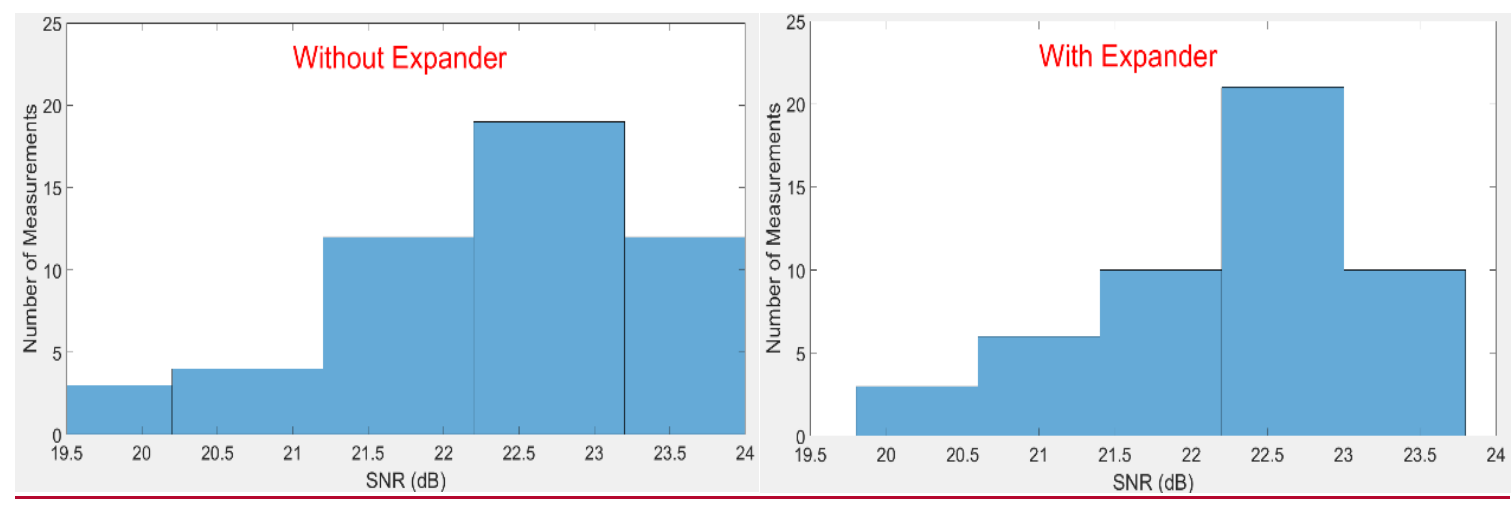

FigURE 57: HISTOGRAMS OF SNR FOR 50 COLLECTED MEASUREMENTS WITHOUT USING THE EXPANDER (TRANSDUCER TILES WERE DIRECTLY CONNECTED TO THE DAS) AND 50 COLLECTED MEASUREMENTS WITH USING THE EXPANDER.

Average SNR for 50 measuremnts without using the expander was $22.35 \mathrm{~dB}$. By connecting the expander to the tiles, this number decreased to $22.31 \mathrm{~dB}$. While the 
expander was connected to the system, four more similar experiments at different power levels were performed to further charactrize the expander (Figure 58).

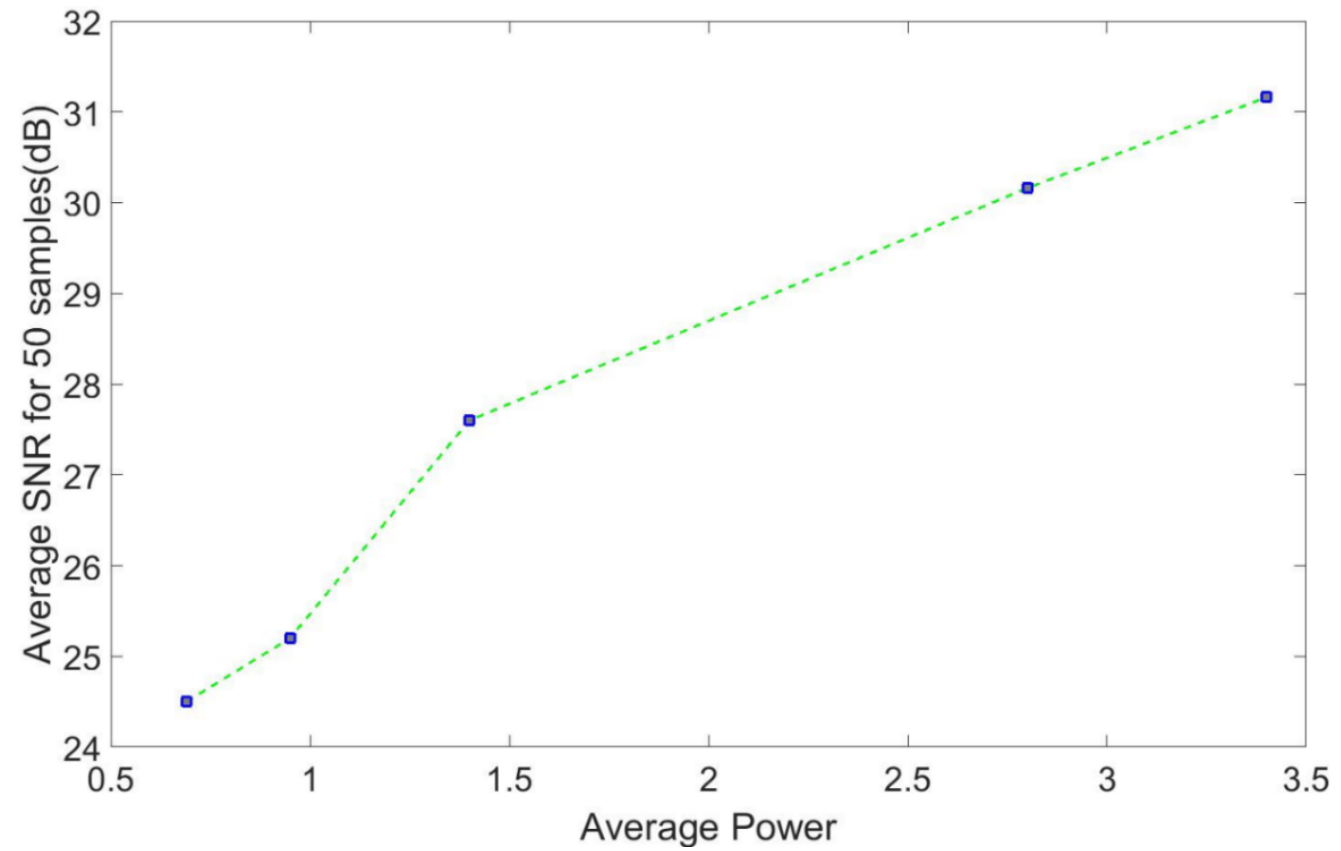

FIGURE 58: EACH DOT IN THE GRAPH SHOWS THE AVERAGE CALCULATED SNR FOR 50 MEASUREMENTS AFTER CONNECTING THE EXPANDER TO THE TRANSDUCER TILES. THE AVERAGE RECEIVING SIGNAL POWER WAS INCREASED

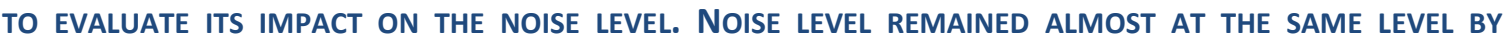
INCREASING THE POWER.

Average signal power was increased from $0.7 \mathrm{~W}$ to $3.4 \mathrm{~W}$ in 5 steps and the average SNR was calculated from 50 measurements at each step while the expander was connected to the system. The results show that the level of the detected noise remain the same while the power is increasing.

The noise was composed of primarily high frequency components. But, grounding the shields of the cables and using appropriate filters would highly drop the noise (Figure 59). 

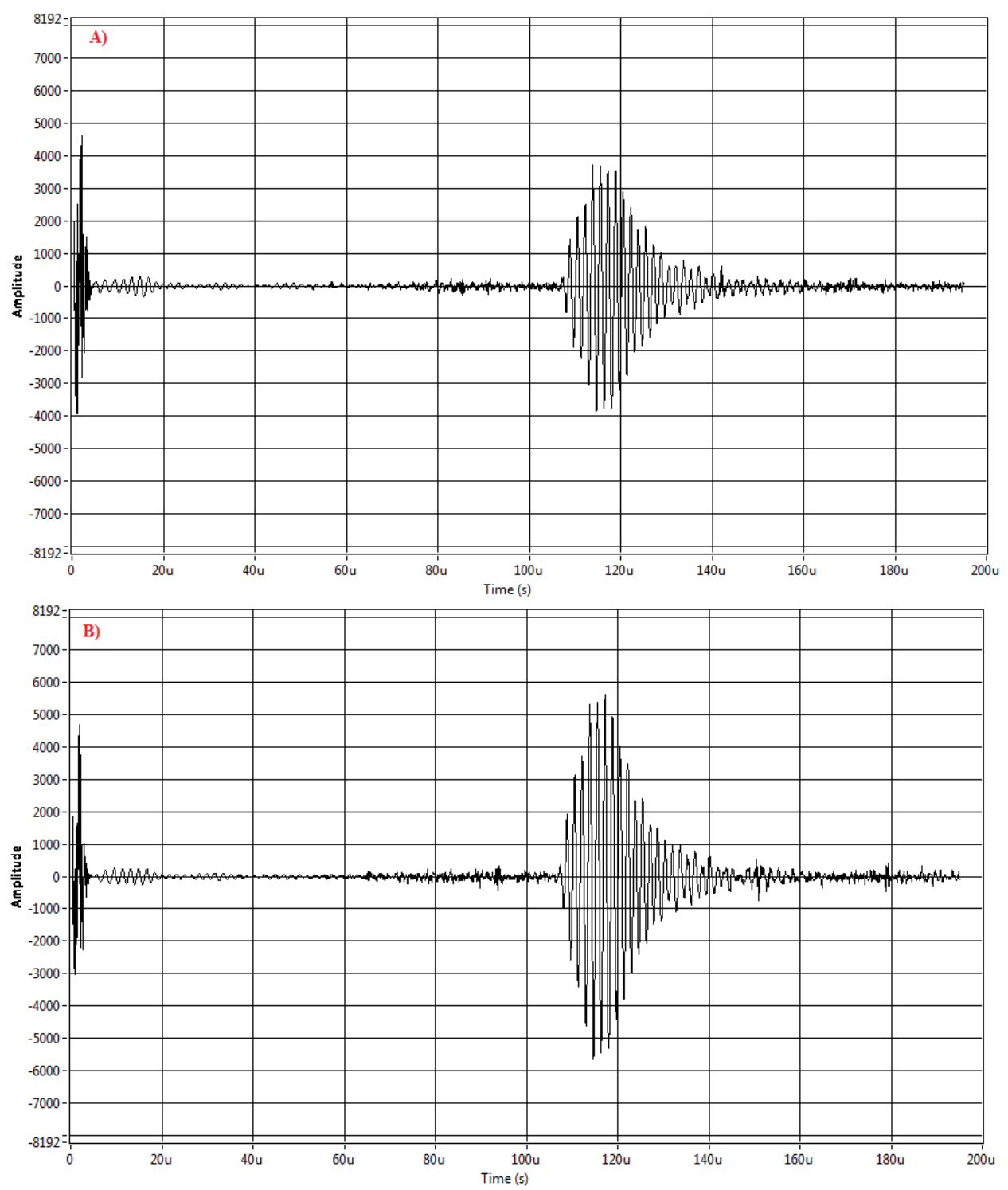

FIGURE 59: THE HIGH PASS FILTER WITH THE STOP FREQUENCY OF 0.2 MHZ AND THE LOW PASS FILTER WITH THE STOP FREQUENCY OF 3.5 MHZ. RECEIVED ULTRASONIC SIGNALS COLLECTED FROM ONE OF THE ELEMENTS OF THE ARRAY DIRECTLY FROM DAS (A) AND AFTER PASSING THROUGH THE EXPANDER (B) AFTER APPLYING FILTERS. THE VERTICAL AXIS SHOWS THE PEAK-TO-PEAK AMPLITUDE OF THE SIGNAL IN MILLIVOLT AND THE HORIZONTAL AXIS SHOWS THE DURATION OF THE SIGNAL IN MICROSECOND.

The data and control acquisition system is capable to apply high pass and low pass filters. For our application, single frequency sending and receiving signals is important. Therefore, the range of the filtering spectrum should be selected in a way that it is not affecting $0.5 \mathrm{MHz}$. The received signals in Figure 58 are achieve without filtering. Figure 59 shows the same signals after applying the high pass filter with the 
stop frequency of $0.2 \mathrm{MHz}$ and the low pass filter with the stop frequency of $3.5 \mathrm{MHZ}$. The frequency spectrum of the fabricated tile elements show that these cut off frequencies are far from their functioning resonance frequency of $500 \mathrm{kHz}$ (Figure 60).
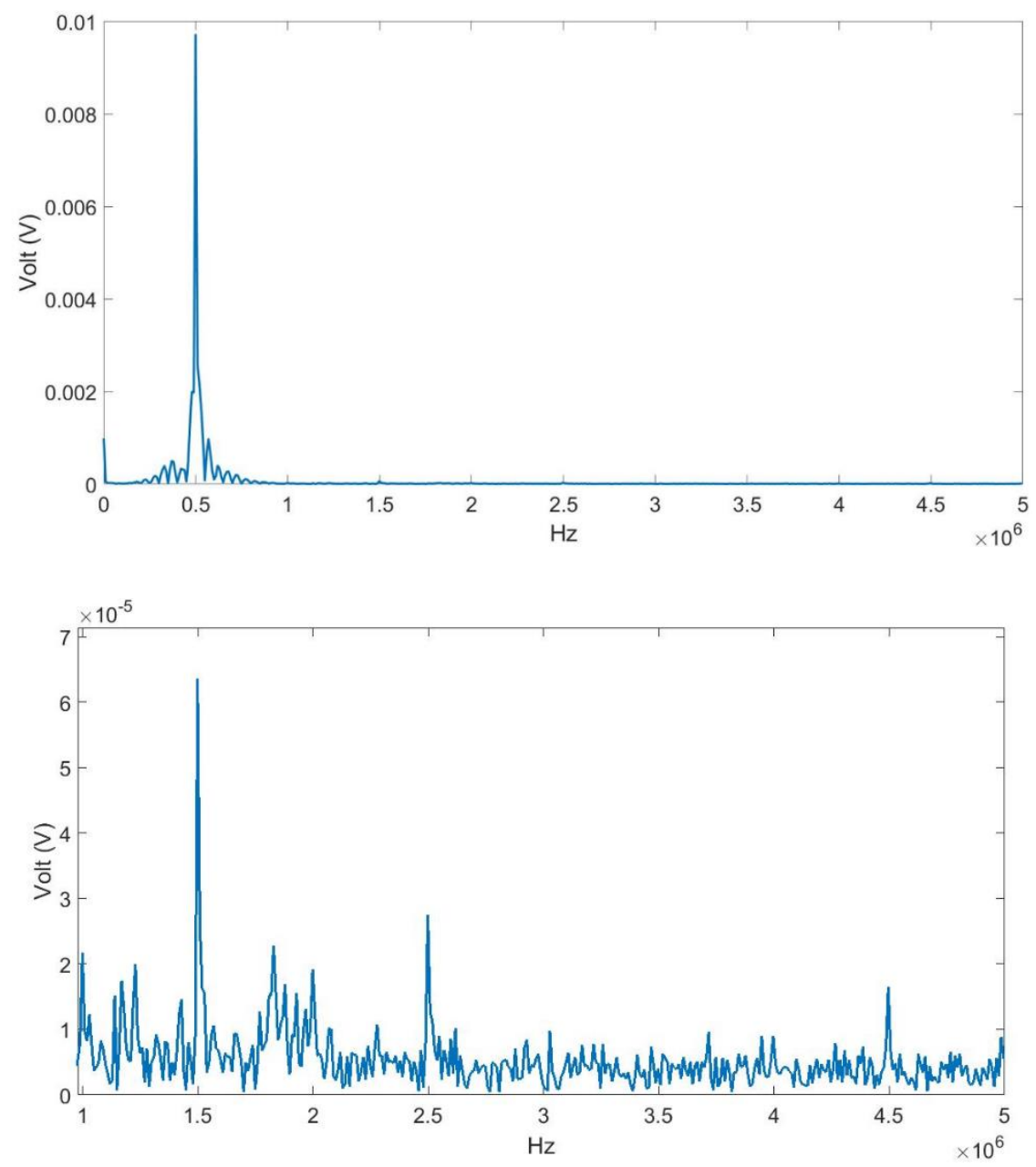

FIGURE 60: FREQUENCY RESPONSE OF A FUNCTIONING TRANSDUCER ELEMENT. HIGHEST AMPLITUDE WAS MEASURED AT THE RESONANCE FREQUENCY OF THE FUNCTIONING ELEMENT (UPPER FIGURE). THE NOISE WAS COMPOSED OF PRIMARILY HIGH FREQUENCY COMPONENTS (LOWER FIGURE).

Figure 60 shows the frequency spectrum of a measured propagating pressure signal in the water in front of an activated element by a square pulses at $500 \mathrm{KHz}$. Details of signal measurement process explained in Section 4.3. The figure clearly shows the peak amplitude at the resonance frequency $(500 \mathrm{kHz})$ of the element. As the array is 
intended to later perform a single frequency tomographic data collection from the brain to be used in the new image reconstruction algorithm [1], the cut of frequency of the low pass filter could be chose low enough to eliminate the high frequency noise components.

In order to check the capability of the array for intended application, a cylindrical testing assembly was designed and fabricated (Figure 61).

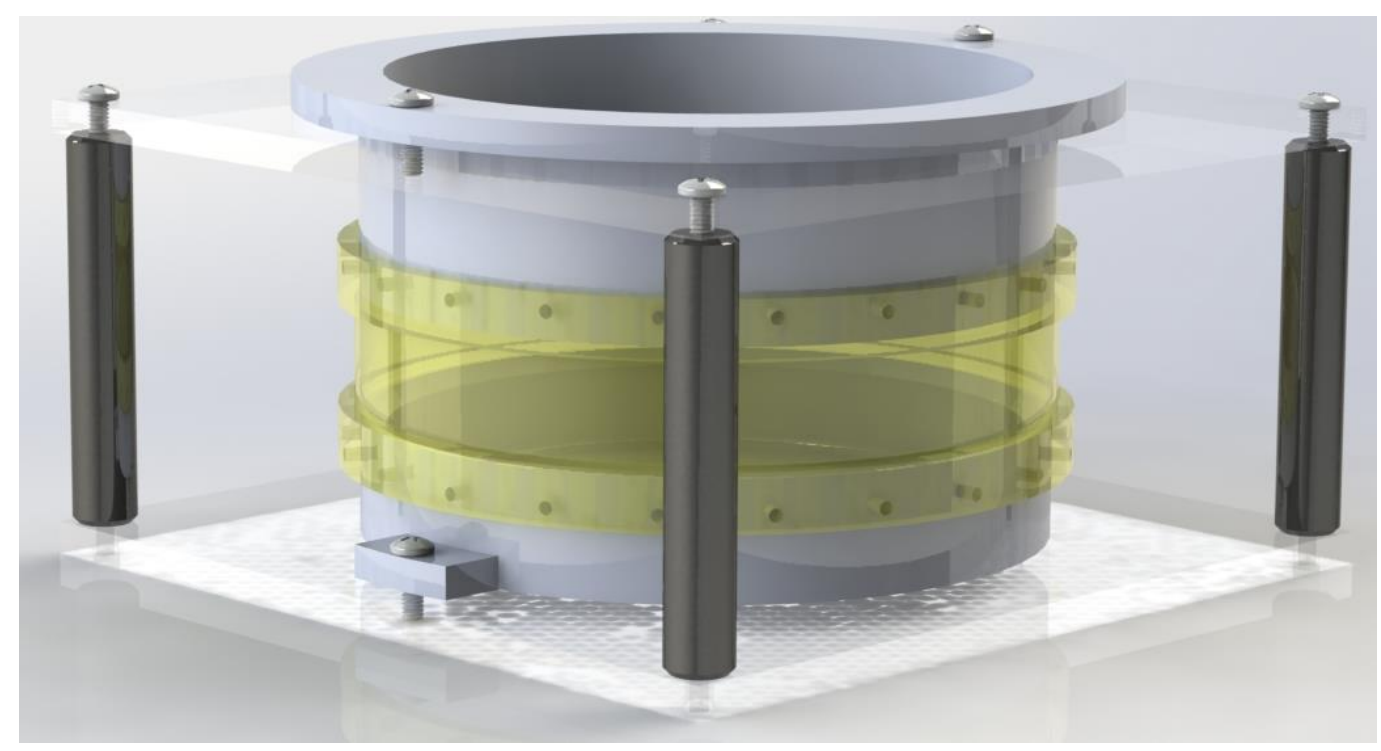

FIGURE 61: CYLINDRICAL TESTING ASSEMBLY. THIS ASSEMBLY IS DESIGNED BY SOLIDWORKS AND MADE BY USING 3D PRINTER TO TIGHTLY FASTEN THE TRANSDUCERS AROUND THE SPONGE.

Then, transducer array is fastened around a circular shape sponge filled with water (Figure 62). 


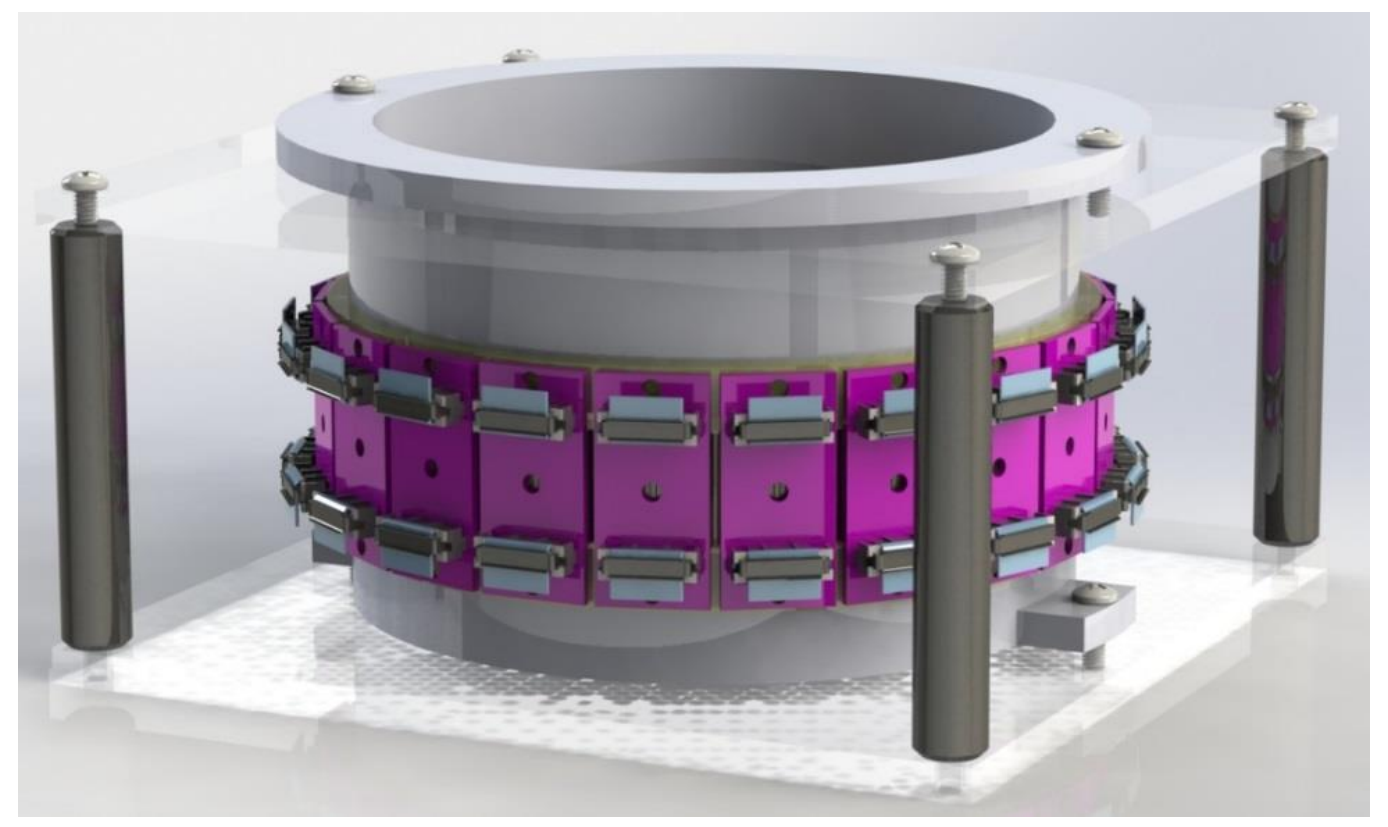

FIGURE 62: CONNECTING TRANSDUCERS TO CYLINDRICAL TESTING ASSEMBLY WITH THE SPONGE IN THE MIDDLE. SO THE TRANSDUCERS SURROUND THE CIRCULAR SPONGE.

In this experimental setup, 21 transducers were mounted around the sponge and the whole setup was fixed on a table to avoid the displacement of the elements. Finally, data was collected in the way called full matrix capture. Every time that the control and data acquisition system sent the signal from each element, the received scattered signals were collected by switching between the transducers elements. Ten cycles of square pulse signal at $40 \mathrm{Vp}-\mathrm{p}$ with frequency of 500k were defined to send the signals in FMC mode as explained in details in Chapter 4. All MUXs were controlled using Arduino Due ${ }^{\circledR}$. Figure 63 shows the results of the collected signals using the experimental setup shown in Figure 62. 


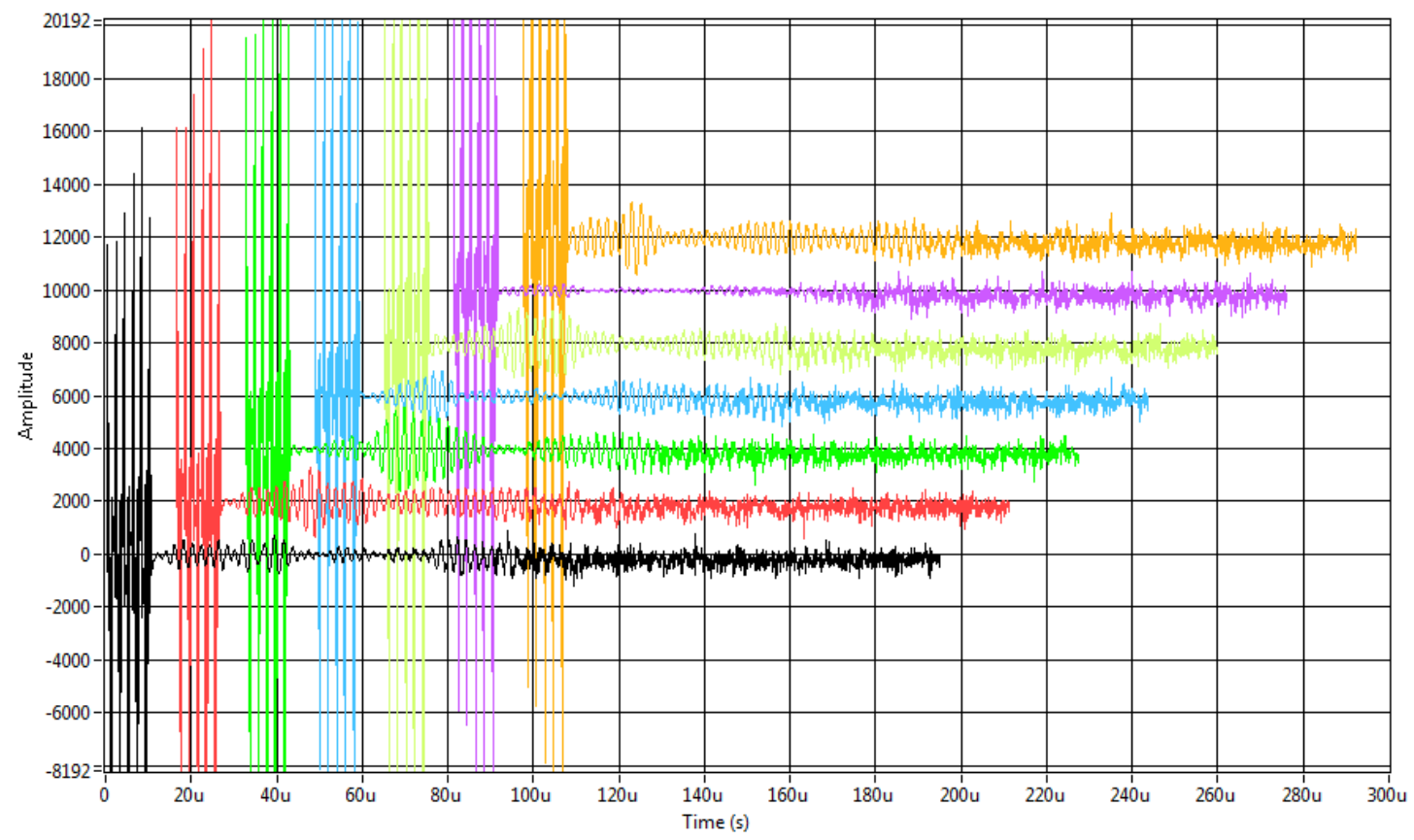

FIGURE 63: RECEIVED SCATTERED ULTRASONIC SIGNALS USING THE ARRAY. THE PICTURE SHOWS RECEIVING SIGNALS BY SEVEN ELEMENTS OF DIFFERENT TRANSDUCERS AROUND A CIRCULAR SPONGE. THE VERTICAL AXIS SHOWS THE PEAK-TO-PEAK AMPLITUDE OF THE SIGNAL IN MILLIVOLT AND THE HORIZONTAL AXIS SHOWS THE DURATION OF THE SIGNAL IN MICROSECOND.

The image reconstruction algorithm is based on the new concept of diffraction tomography [1]. It shows the reconstruction of a 2D slice image of any curved shape plane by surrounding the plane with ultrasound transmitters and receivers. The imaging process starts with the measurement of acoustic signals over an array of sources/receivers on a closed curve that surrounds a region of interest (ROI). The way of collecting signal around the ROI is called full matrix capture. As explained in details in Chapter 2, in full matrix capture, one element fires the signal and all other elements record the scattered field around the ROI. This process would be repeated for all the elements till the last element fires the signal and all the elements record the scattered field. The position of the elements should be known. Vectors normal to the curve should be determined. This could be done by taking the cross product between tangential vectors (differences between 
neighboring position vectors) and a vector normal to the imaging plane. By determining appropriate Green's functions, and rotation angle, received data is projected to a virtual line exterior to the curve.

In the next step, the received and projected data is associated to a new signal on the original curve by reciprocity action. Then, this signal would be projected to a receiver line parallel to the source line but on the opposite side of the object. This process could be repeated multiple times at different angles by using the original received dataset [1]. 


\section{CHAPTER VI}

\section{CONCLUSION AND FUTURE WORK}

Step-by-step designing and testing the transducers were explained before. The results of the design was used to make an array of transducers that would conform around

the head (Figure 1). The array is capable of getting 2D images of the brain. Each transducer is a 20x20 mm composite plate, made of $65 \%$ lead zirconate titanate (PZT) 13 piezoelectric ceramic fibers. The resonance frequency of the transducers is $0.5 \mathrm{MHz}$, and the design was considered to be light and pliable enough to provide optimal coupling to the head. The PC board design was done using the SolidWorks ${ }^{\circledR}$ and Eagle software ${ }^{\circledR}$ and connected to PZT through conductive epoxy. Then, a very low viscosity, "wicking" epoxy injected between the surfaces of PC board and PZT to make a strong PZT-PC board connection. Finally, the assembly of PZT-PC board would be mounted on a flexible 'headband' with the thickness of $0.5 \mathrm{~mm}$ that is composed of a 3D printed, soft, polyjet material which will wrap around the head. 
Three transducers were tested to evaluate the propagated planes caused by vibrations of transducer surface. Pressure fields were measured at specific distances from the surface of the transducer using hydrophone in water. The measured plane was perpendicular to the propagation axis and parallel to the transducer surface. Then, the plane was back-projected to the surface using projection algorithm. Results showed that each element is finely separated from each other and there is less than $3 \mathrm{~dB}$ decrease in the pressure amplitude of each element compared to the element with the largest amplitude.

The imaging system is composed of around 500 sending and receiving elements. But the data control and acquisition system supports up to 128 elements. Therefore, an expander was designed and fabricated to cover more number of elements of about 500 . Six PCB was designed and fabricated to be functioned as an expander between the data and control acquisition system and the elements of the transducers. Each PCB has 19 MUXs. Each channel of the data acquisition system could be connected to the output of one MUX. The multiplexers are dual 4-channel bidirectional analog multiplexers capable to switch up to $72 \mathrm{v}( \pm 36)$. Therefore, every four transducer elements are connected to the inputs of the multiplexers (Figure 50). As each PCB has 19 MUXs, a total of 76 number of elements could be connected to each PCB. The total of 6 PCBs are designed to cover up to 456 elements of the transducer plates. Arduino Due® is used for controlling the switching between the channels of MUXs.

The electrical characteristics of the expander were evaluated by addressing the level of noise and crosstalk in all MUXs. The results of the experiments show the crosstalk of about $-58.7 \pm 2.9 \mathrm{~dB}$ in MUX inputs when they are not connected to the 
output channels. Such a result is compared to the nearby input which is connected to the out channel and passing the transmitted signal. The level of noise in the detected signals and SNR are evaluated before and after connecting the expander to the data and control acquisition system. The noise could be eliminated by shielding the ribbon cables and the expander as well as defining appropriate low pass and high pass filters (Figure 59 and Figure 60).

The final goal of this project was to overcome skull attenuation by applying sufficiently low frequency beams to penetrate maximum energy through skull and designing a transducer array to receive the scattered signals all around the imaging plane. Even in the relatively low frequency range of $0.5 \mathrm{MHz}$ to $1 \mathrm{MHz}$ only about $10 \%$ of an acoustic signal (power) is transmitted through the skull [24]-[26]. Therefore, in a pulseecho scenario signals on the order of $1 \%$ of its soft-tissue equivalent would be expected.

The new technique of ultrasound diffraction tomography and inverse scattering is based on a single low frequency ultrasound which would be applied to any arbitrarilyshaped curve of sources and receivers [1]. Therefore, for intended use in this project of brain imaging, it allows us to provide coupling to the head by attaching the transducers to the skull skin. Moreover, using a single frequency ultrasound would allow us to deliver more energy at a single frequency and to enhance receivers' sensitivity for collecting the scattered signals at that frequency. This method was tested on three different irregular curves, in addition to a Shepp-Logan phantom, by using two virtual source/receiver pairs at $0.5 \mathrm{MHz}$ frequency (the sound speed similar to the range of many fluids and tissues). The results showed that point-scatters inside the surrounding elements could be identified with low distortion, but with little image contrast [1]. The new technique was developed 
to reconstruct the image around irregularly-shaped boundaries. It could be applied to any boundary shapes, especially in the situations where long boundary needs to be covered by sources and receivers to reconstruct an image. Therefore, an array of transducers was made to satisfy all the requirements needed for brain imaging by applying the new image reconstruction algorithm [1]. The array is flexible to provide optimal coupling to the nonsymmetrical shape of the human head. Moreover, it consists of elements with the highest sensitivity to their resonance frequency, as the algorithm works only with single frequency. Therefore, the transducers can deliver more energy at a single frequency. They have enough sensitivity for that frequency to receive the scattered signal in the brain. In addition, this would be a phased array of transducers around a plane of the head (to be imaged later) to perform FMC from different angles of the head.

The way of collecting data in diffraction tomography technique is called full matrix capture (FMC). In FMC, one of the elements of the array would fire the signal and all of the elements would detect the received scattered signal. This would be repeated till all the elements would fire the signal once (see Chapter 2 for details). As the algorithm is based on a single frequency, the resolution would be increased by transmitting data from different angles of transmitting the signal.

The new ultrasound imaging system for the brain would provide major advantages over other image modalities. First, compared to CT and MRI, ultrasound imaging systems are portable, more cost-effective, and it provides real time monitoring of the image (depending on the image reconstruction algorithm). Therefore, it would be a pivotal means of imaging when early detection of problems is important, especially when access to other image modalities is limited and the resolution of the image is not that 
critical. Brain bleeding (hemorrhages or cerebral hemorrhages) which happens during an injury, for example, can be detected by the new portable ultrasonic imaging system in the ambulance (Figure 2).

In order to test the feasibility of the array in detecting $2 \mathrm{D}$ images of the brain by using the new imaging reconstruction technique, the array should be tested in real conditions. Registration of the elements could be done by having a CT scan of the head and using a 3D scanner to find the location of each element (Figure 64).

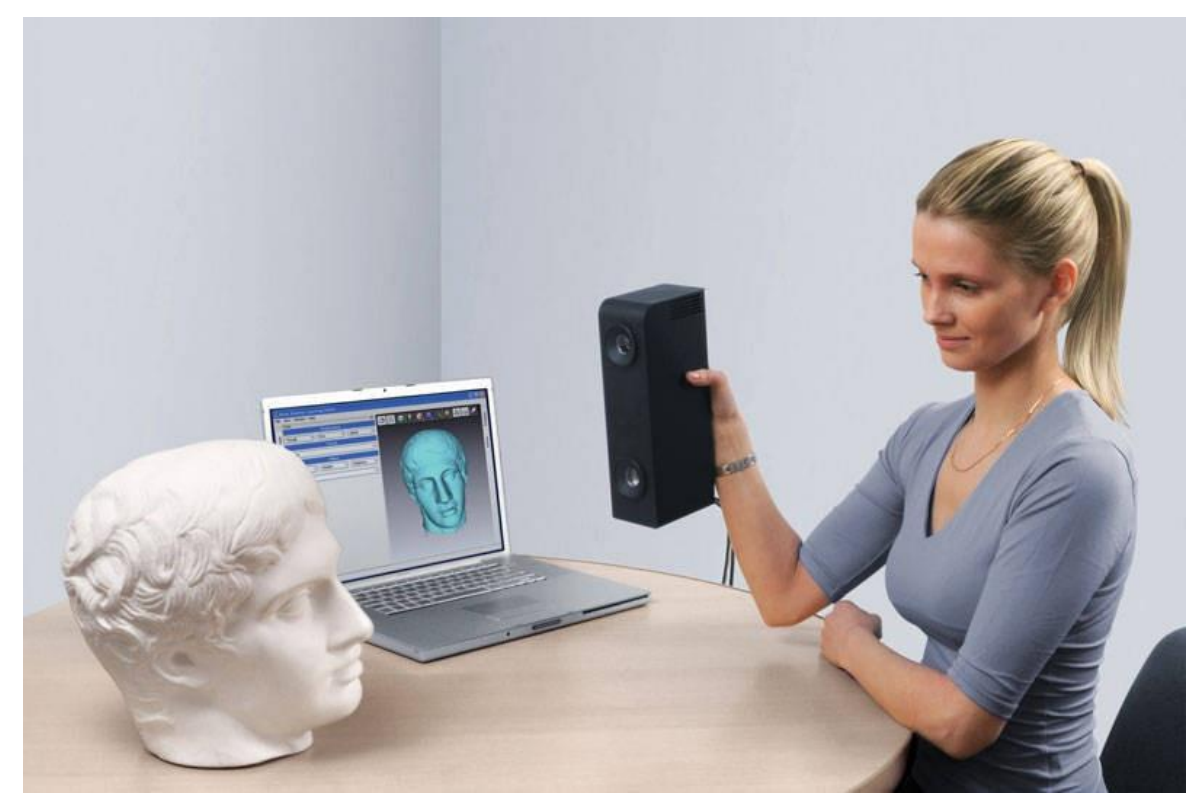

FIGURE 64: USING 3D SCANNER TO FIND THE LOCATION OF EACH TRANSDUCER. TAKEN FROM [187].

By using a 3D scanner and simply scanning around the head, where the array is fastened, we can have the exact location of the elements. In addition, by having the CT scan of the head, skull distortion can be corrected as well. Therefore, the possible next step could be the imaging of the brain phantom, which acoustically mimic the properties of the human head (Figure 65). 


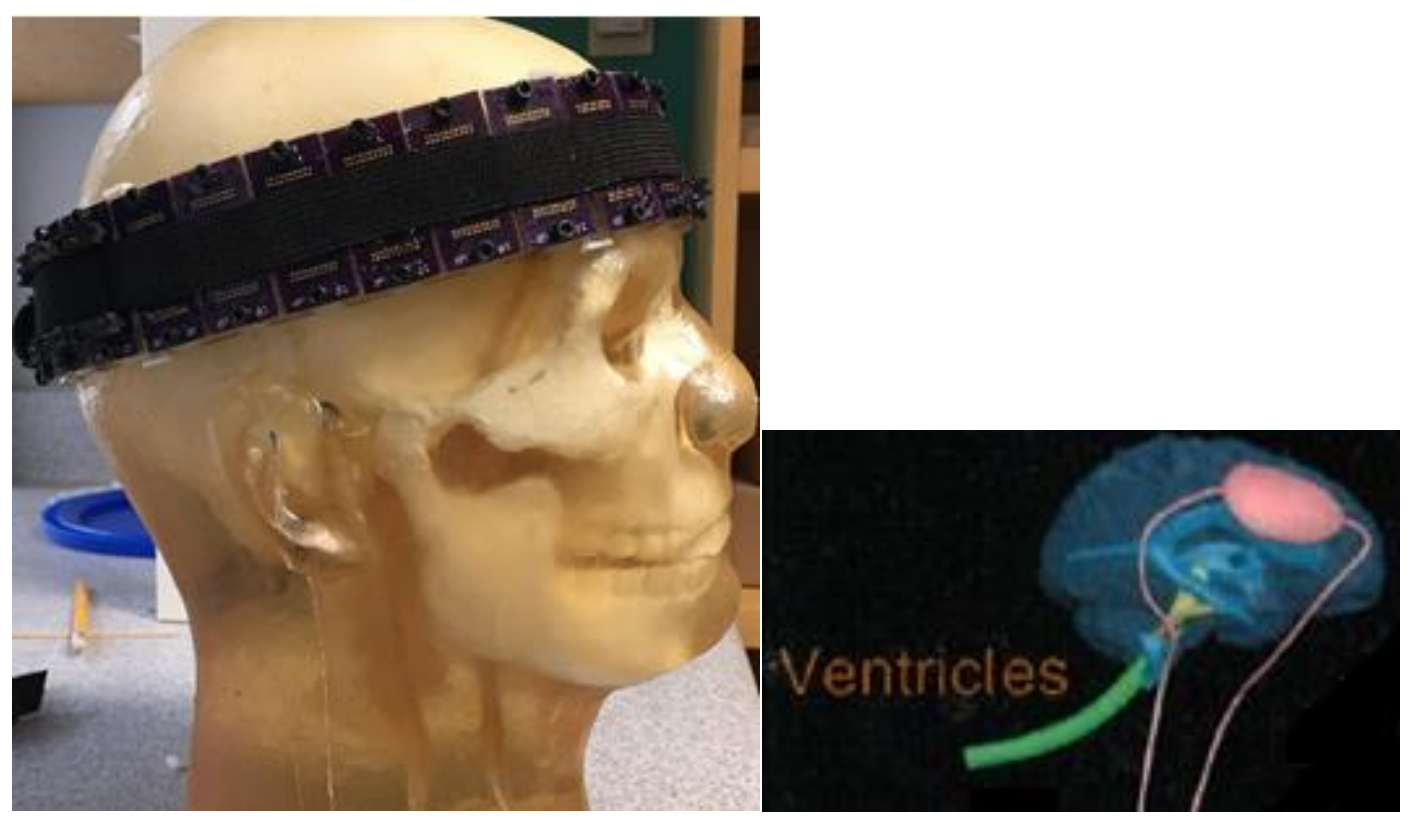

FIGURE 65 : BRAIN PHANTOM. THIS BRAIN PHANTOM ACOUSTICALLY MIMIC THE PROPERTIES OF THE HUMAN HEAD: VeNTRICLES MADE BY 3D PRINTED MATERIALS, CORTICAL BONE MATERIAL FOR SKULL BONE, TRABECULAR BONE MATERIAL FOR TRABECULAR LAYER, ACOUSTICALLY CORRECT SKIN-LIKE TISSUE FOR SKIN. 


\section{REFERENCES:}

[1] G. Clement, "A projection-based approach to diffraction tomography on curved boundaries," Inverse Probl., vol. 30, no. 12, pp. 1-18, 2014.

[2] K. Hynynen and G. Clement, "Clinical applications of focused ultrasound - the brain,” Int. J. Hyperth., vol. 23, no. 2, pp. 193-202, 2007.

[3] G. Clement, P. White, and R. King, "A magnetic resonance imaging-compatible, large-scale array for trans-skull ultrasound surgery and therapy," ultrasound in Medicine, vol. 24, no. 8, pp. 1117-25, 2005.

[4] K. Hynynen, N. McDannold, G. Clement, and F. Jolesz, "Pre-clinical testing of a phased array ultrasound system for MRI-guided noninvasive surgery of the brain—a primate study," Eur. J. Radiology, vol. 59, no. 2, pp. 149-156, 2006.

[5] W. Fry and F. Fry, "Fundamental neurological research and human neurosurgery using intense ultrasound," IRE Trans. Med. Electron., vol. ME-7, no. 3, pp. 166$181,1960$.

[6] W. Fry, "Ultrasound in neurology," Neurology, vol. 6, no. 10, pp. 693-704, 1956.

[7] M. Daffertshofer and M. Fatar, "Therapeutic ultrasound in ischemic stroke treatment: experimental evidence," Eur. J. ultrasound, vol. 16, no. 1-2, pp. 121$130,2002$.

[8] R. Mikulik and A. Alexandrov, "Acute stroke: therapeutic transcranial Doppler sonography," Handb. Neurovascular Ultrasound, vol. 21, pp. 150-160, 2006.

[9] H. Kawata, N. Naya, Y. Takemoto, and S. Uemura, "Ultrasound accelerates 
thrombolysis of acutely induced platelet-rich thrombi similar to those in acute myocardial infarction," Circulation, vol.71, no. 10, pp. 1643-48, 2007.

[10] K. Hynynen, N. McDannold, N. Vykhodtseva, and F. Jolesz, "Noninvasive MR Imaging-guided Focal Opening of the Blood-Brain Barrier in Rabbits 1," Radiology, vol. 220, no. 3, pp. 640-6, 2001.

[11] T. Robinson and P. Lele, "An Analysis of Lesion Development in the Brain and in Plastics by High-Intensity Focused Ultrasound at Low-Megahertz Frequencies,” J. Acoust. Soc., vol. 51, no. 4B, 1972.

[12] W. Fry, W. M. Jr, J. Barnard, and F. Fry, "Production of focal destructive lesions in the central nervous system with ultrasound," J. Neurosurg., vol. 11, no. 5, pp. 471-8, 1954.

[13] F. Fry, S. Goss, and J. Patrick, "Transkull focal lesions in cat brain produced by ultrasound," J. Neurosurg., vol. 54, no. 5, pp. 659-63, 1981.

[14] K. Hynynen, N. McDannold, and N. Vykhodtseva, "Focal disruption of the bloodbrain barrier due to $260-\mathrm{kHz}$ ultrasound bursts: a method for molecular imaging and targeted drug delivery," Neurosurg., vol. 105, no. 3, pp. 445-54, 2006.

[15] M. Kinoshita and N. McDannold, "Noninvasive localized delivery of Herceptin to the mouse brain by MRI-guided focused ultrasound-induced blood-brain barrier disruption," Proc. PNAS, vol. 103, no. 31, pp. 11719-23, 2006.

[16] F. Fry and S. Goss, "Further studies of the transkull transmission of an intense focused ultrasonic beam: Lesion production at $500 \mathrm{kHz}$," Ultrasound Med. Biol., 
vol. 6, no. 1, pp. 33-35, 1980.

[17] K. Hynynen and F. Jolesz, "Demonstration of potential noninvasive ultrasound brain therapy through an intact skull," Ultrasound Med. Biol., vol. 24, no. 2, pp. 275-83, 1998.

[18] G. Clement, J. White, and K. Hynynen, "Investigation of a large-area phased array for focused ultrasound surgery through the skull," Phys. Med., vol. 45, no. 4, pp. $1071-83,2000$.

[19] N. Vykhodtseva, K. Hynynen, and C. Damianou, "Histologic effects of high intensity pulsed ultrasound exposure with subharmonic emission in rabbit brain in vivo," Ultrasound Med., vol. 21, no. 7, pp. 969-79, 1995.

[20] S. Umemura, N. Yumita, and R. Nishigaki, "Sonochemical activation of hematoporphyrin: a potential modality for cancer treatment," Ultrasonics Symp Proc, 1989.

[21] K. Hynynen, V. Colucci, A. Chung, and F. Jolesz, "Noninvasive arterial occlusion using MRI-guided focused ultrasound," Ultrasound Med., vol. 22, no. 8, pp. 10717, 1996.

[22] N. McDannold, G. T. Clement, P. Black, F. Jolesz, and K. Hynynen, "Transcranial magnetic resonance imaging- guided focused ultrasound surgery of brain tumors: initial findings in 3 patients.," Neurosurgery, vol. 66, no. 2, pp. 323-32; discussion 332, Feb. 2010.

[23] F. Meral, M. Jafferji, and P. White, "Two-dimensional image reconstruction with 
spectrally-randomized ultrasound signals," IEEE Trans., vol. 60, no. 12, pp. 250110, 2013.

[24] T. Hueter, A. Cavlieri, B. Laugmuir, W. Butkus, and D. Kyrazis, "The detection of intracranial tumors by use of ultrasound," Lab. Massachusetts Inst.Tech, pp. 3846, 1951.

[25] "Real time B-mode echo-encephalography," Ultrasound in Med, pp. 373-82, 1976.

[26] F. Fry, "Transkull transmission of an intense focused ultrasonic beam," Ultrasound Med. Biol., vol. 3, no. 2-3, pp. 179-81, 1977.

[27] C. Pintavirooj and M. Sangworasil, "Ultrasonic Diffraction Tomography," Int. J. Appl. Biomed. Eng., vol. 1, no. 1, pp. 34-40, 2008.

[28] P. Huthwaite, F. Simonetti, and N. Duric, "Combining time of flight and diffraction tomography for high resolution breast imaging: Initial invivo results (L)," J. Acoust. Soc. Am., vol. 132, no. 3, pp. 1249-1252, Sep. 2012.

[29] S. Hua, M. Ding, and M. Yuchi, "Sparse-view ultrasound diffraction tomography using compressed sensing with nonuniform FFT.," Comput. Math. Methods Med., vol. 2014, no. 329350, Apr. 2014.

[30] R. Mueller, M. Kaveh, and G. Wade, "Reconstructive tomography and applications to ultrasonics,” Proc. IEEE, vol. 67, no. 4, pp. 567-87, 1979.

[31] M. S. Avinash C. Kak, Principles of Computerized Tomographic Imaging. Society of Industrial and Applied Mathematics, vol. 29, no. 1, 2001. 
[32] K. Iwata and R. Nagata, "Calculation of Refractive Index Distribution from Interferograms Using the Born and Rytov's Approximation,” Jpn. J. Appl. Phys., vol. 14, no. S1, Jan. 1975.

[33] A. Devaney and G. Beylkin, "Diffraction tomography using arbitrary transmitter and receiver surfaces," Ultrason. Imaging, vol. 6, no. 2, pp. 181-93, 1984.

[34] L. -J. Gelius, I. Johansen, N. Sponheim, and J. J. Stamnes, “A generalized diffraction tomography algorithm," J. Acoust. Soc. Am., vol. 89, no. 2, pp. $523-$ 528, Feb. 1991.

[35] J. Sun and K. Hynynen, "The potential of transskull ultrasound therapy and surgery using the maximum available skull surface area," J. Acoust. Soc., vol. 105, no. $4,1999$.

[36] Y. Jing, F. Meral, and G. Clement, "Time-reversal transcranial ultrasound beam focusing using a k-space method," Phys. Med. Biol., vol. 57, no. 4, pp. 901-17, 2012.

[37] S. Smith, G. Trahey, and O. Von Ramm, "Phased array ultrasound imaging through planar tissue layers," Ultrasound Med. Biol., vol. 12, no. 3, pp. 229-243, 1986.

[38] M. O’Donnell and S. Flax, "Phase aberration measurements in medical ultrasound: Human studies," Ultrason. Imaging, vol. 10, no. 1, 1988.

[39] J. White, G. Clement, and K. Hynynen, "Transcranial ultrasound focus reconstruction with phase and amplitude correction," ieee Trans., vol. 52, no. 9, 
pp. 1518-22, 2005.

[40] G. Clement and K. Hynynen, "Correlation of ultrasound phase with physical skull properties," Ultrasound Med. Biol., vol. 28, no. 5, pp. 617-24, 2002.

[41] G. Clement and K. Hynynen, "A non-invasive method for focusing ultrasound through the human skull," Phys. Med. Biol., vol. 47, no. 8, pp. 1219-36, 2002.

[42] J. Aubry, M. Tanter, M. Pernot, and J. Thomas, "Experimental demonstration of noninvasive transskull adaptive focusing based on prior computed tomography scans," Acoust. Soc., vol. 113, no. 1, 2003.

[43] J. Gâteau, L. Marsac, M. Pernot, and J. Aubry, “Transcranial ultrasonic therapy based on time reversal of acoustically induced cavitation bubble signature," IEEE Trans., vol. 57, no. 1, pp. 134-44, 2010.

[44] G. Clement, "Spectral image reconstruction for transcranial ultrasound measurement," Phys. Med. Biol., vol. 50, no. 23, pp. 5557-72, 2005.

[45] F. Fry and J. Barger, "Acoustical properties of the human skull," J. Acoust. Soc. Am., vol. 63, no. 5, 1978.

[46] P. J. White, "Modalities for optimization of ultrasound transmission through the human skull," Penn State University, , 2005.

[47] G. Clement, J. Sun, and T. Giesecke, "A hemisphere array for non-invasive ultrasound brain therapy and surgery," Phys. Med., vol. 45, no. 12, pp. 3707-19, 2000.

[48] M. Tanter, J. Thomas, and M. Fink, "Focusing and steering through absorbing and 
aberrating layers: Application to ultrasonic propagation through the skull," $J$. Acoust. Soc., vol. 103, no. 5, 1998.

[49] G. Clement and K. Hynynen, "Micro-receiver guided transcranial beam steering," ieee Trans. Ultrason., vol. 49, no. 4, pp. 447-53, 2002.

[50] C. Connor and G. Clement, "A unified model for the speed of sound in cranial bone based on genetic algorithm optimization," Phys. Med., vol. 47, no. 22, pp. 3925-44, 2002.

[51] G. Clement and K. Hynynen, "Criteria for the design and characterization of largearea arrays for transskull ultrasound surgery and therapy," Ultrason. Symp. 2000 IEEE, 2000.

[52] S. L. James, "Transcranial Ultrasound as a Potential Modality for Real-Time Observation of Brain Motion," Cleveland State University, 2017.

[53] “A Cyberphysics Page.” [Online]. Available: http://www.cyberphysics.co.uk/Q\&A/KS5/medical/ultrasound/questions.html. [Accessed: 18-Aug-2017].

[54] “Image Gallery transducer.” [Online]. Available: http://keywordsuggest.org/gallery/852656.html. [Accessed: 18-Aug-2017].

[55] “NDK - NIHON DEMPA KOGYO CO., LTD.” [Online]. Available: http://www.ndk.com/en/sensor/ultrasonic/basic02.html. [Accessed: 18-Aug-2017].

[56] “Characteristics of Piezoelectric Transducers.” [Online]. Available: https://www.nde- 
ed.org/EducationResources/CommunityCollege/Ultrasonics/EquipmentTrans/char acteristicspt.htm. [Accessed: 20-Sep-2017].

[57] “Transducers. - ppt download.” [Online]. Available: http://slideplayer.com/slide/4642627/. [Accessed: 20-Sep-2017].

[58] “TRANSDUCER ARRAYS - tritonbkrzysztofczyk.” [Online]. Available: https://sites.google.com/site/tritonbkrzysztofczyk/transducer-arrays. [Accessed: 20-Sep-2017].

[59] "Physics and Instrumentation in Doppler and B-mode Ultrasonography | Radiology Key.” [Online]. Available: https://radiologykey.com/physics-andinstrumentation-in-doppler-and-b-mode-ultrasonography/. [Accessed: 20-Sep2017].

[60] “Longitudinal and Transverse Wave Motion.” [Online]. Available: http://www.acs.psu.edu/drussell/Demos/waves/wavemotion.html. [Accessed: 15Sep-2017].

[61] “2.3 Wave Propagation | Olympus IMS.” [Online]. Available: http://www.olympus-ims.com/en/ndt-tutorials/flaw-detection/wave-propagation/. [Accessed: 15-Sep-2017].

[62] E. Braunwald and R. O. Bonow, "Braunwald's heart disease : a textbook of cardiovascular medicine." BMH. Med. Journal, vol. 5, no. 2, 2018.

[63] "Ultrasonic Testing Using Phased Arrays." [Online]. Available: http://www.ndt.net/article/wcndt00/papers/idn151/idn151.htm. [Accessed: 19-Sep- 
2017].

[64] "Reflection, Refraction, Scattering and Attenuation | VAULT." [Online]. Available: http://www.vaultrasound.com/educational-resources/ultrasoundphysics/reflection-refraction/. [Accessed: 19-Sep-2017].

[65] "The effects of ultrasound on different tissue types (practice) | Khan Academy." [Online]. Available: https://www.khanacademy.org/test-prep/mcat/physicalsciences-practice/physical-sciences-practice-tut/e/the-effects-of-ultrasound-ondifferent-tissue-types. [Accessed: 19-Sep-2017].

[66] Y. Ishihara et al., "A precise and fast temperature mapping using water proton chemical shift.," Magn. Reson. Med., vol. 34, no. 6, pp. 814-23, Dec. 1995.

[67] J. Y. Chapelon, M. Ribault, F. Vernier, R. Souchon, and A. Gelet, "Treatment of localised prostate cancer with transrectal high intensity focused ultrasound.," Eur. J. Ultrasound, vol. 9, no. 1, pp. 31-8, Mar. 1999.

[68] N. T. Sanghvi et al., "Noninvasive surgery of prostate tissue by high intensity focused ultrasound: an updated report.," Eur. J. Ultrasound, vol. 9, no. 1, pp. 1929, Mar. 1999.

[69] C. M. C. Tempany, E. A. Stewart, N. McDannold, B. J. Quade, F. A. Jolesz, and K. Hynynen, "MR Imaging-guided Focused Ultrasound Surgery of Uterine Leiomyomas: A Feasibility Study,” Radiology, vol. 226, no. 3, pp. 897-905, Mar. 2003.

[70] F. Wu et al., "Pathological changes in human malignant carcinoma treated with 
high-intensity focused ultrasound.," Ultrasound Med. Biol., vol. 27, no. 8, pp. 1099-106, Aug. 2001.

[71] R. Medel et al., "Magnetic resonance-guided focused ultrasound surgery: Part 2: A review of current and future applications.," Neurosurgery, vol. 71, no. 4, pp. 75563, Oct. 2012.

[72] F. A. Jolesz, "MRI-Guided Focused Ultrasound Surgery," Annu. Rev. Med., vol. 60, no. 1, pp. 417-430, Feb. 2009.

[73] M. W. Dewhirst, B. L. Viglianti, M. Lora-Michiels, M. Hanson, and P. J. Hoopes, "Basic principles of thermal dosimetry and thermal thresholds for tissue damage from hyperthermia," Int. J. Hyperth., vol. 19, no. 3, pp. 267-294, Jan. 2003.

[74] S. A. Sapareto and W. C. Dewey, "Thermal dose determination in cancer therapy.," Int. J. Radiat. Oncol. Biol. Phys., vol. 10, no. 6, pp. 787-800, Jun. 1984.

[75] W. L. Nyborg, "Biological effects of ultrasound: development of safety guidelines. Part II: general review.," Ultrasound Med. Biol., vol. 27, no. 3, pp. 301-33, Mar. 2001.

[76] K. Hynynen, "Demonstration of enhanced temperature elevation due to nonlinear propagation of focussed ultrasound in dog's thigh in vivo.," Ultrasound Med. Biol., vol. 13, no. 2, pp. 85-91, Feb. 1987.

[77] K. Hynynen, "The threshold for thermally significant cavitation in dog's thigh muscle in vivo.," Ultrasound Med. Biol., vol. 17, no. 2, pp. 157-69, 1991.

[78] V. A. Khokhlova, M. R. Bailey, J. A. Reed, B. W. Cunitz, P. J. Kaczkowski, and 
L. A. Crum, "Effects of nonlinear propagation, cavitation, and boiling in lesion formation by high intensity focused ultrasound in a gel phantom.," J. Acoust. Soc. Am., vol. 119, no. 3, pp. 1834-48, Mar. 2006.

[79] R. Cleveland and J. McAteer, "The physics of shock wave lithotripsy," Smith's Textb. Endourol., 2007.

[80] G. Ter Haar and C. Coussios, "High intensity focused ultrasound: physical principles and devices.," Int. J. Hyperthermia, vol. 23, no. 2, pp. 89-104, Mar. 2007.

[81] K. Hynynen et al., "500-element ultrasound phased array system for noninvasive focal surgery of the brain: A preliminary rabbit study with ex vivo human skulls," Magn. Reson. Med., vol. 52, no. 1, pp. 100-107, Jul. 2004.

[82] C. W. Connor, G. T. Clement, and K. Hynynen, "A unified model for the speed of sound in cranial bone based on genetic algorithm optimization.," Phys. Med. Biol., vol. 47, no. 22, pp. 3925-44, Nov. 2002.

[83] K. Hynynen and F. A. Jolesz, "Demonstration of potential noninvasive ultrasound brain therapy through an intact skull.," Ultrasound Med. Biol., vol. 24, no. 2, pp. 275-83, Feb. 1998.

[84] N. McDannold, R. L. King, and K. Hynynen, "MRI monitoring of heating produced by ultrasound absorption in the skull: In vivo study in pigs," Magn. Reson. Med., vol. 51, no. 5, pp. 1061-1065, May 2004.

[85] N. McDannold, N. Vykhodtseva, F. A. Jolesz, and K. Hynynen, "MRI 
investigation of the threshold for thermally induced blood-brain barrier disruption and brain tissue damage in the rabbit brain," Magn. Reson. Med., vol. 51, no. 5, pp. 913-923, May 2004.

[86] N. Vykhodtseva, V. Sorrentino, F. A. Jolesz, R. T. Bronson, and K. Hynynen, "MRI detection of the thermal effects of focused ultrasound on the brain.," Ultrasound Med. Biol., vol. 26, no. 5, pp. 871-80, Jun. 2000.

[87] N. Mcdannold, G. Clement, P. Black, F. Jolesz, and K. Hynynen, "Transcranial MRI-guided focused ultrasound surgery of brain tumors: Initial findings in three patients," Neurosurgery, vol. 66, no. 2, pp. 323-32, 2010.

[88] P. Azimi, "Transcranial Focused Ultrasound in Functional Neurosurgery for Essential Tremor and Parkinson's Disease: A Review Article," Int. Clin. Neurosci. J., vol. 3, no. 3, pp. 127-137, Dec. 2016.

[89] “Essential Tremor, Tremor Dominant Parkinson's Disease, Neuropathic Pain Treatment | INSIGHTEC.” [Online]. Available: https://www.insightec.com/clinical/neurosurgery. [Accessed: 25-Sep-2017].

[90] O. Oralkan et al., "Capacitive Micromachined Ultrasonic Transducers: NextGeneration Arrays for Acoustic Imaging," ieee Trans. Ultrason. Ferroelectr. Freq. Control, vol. 49, no. 11, 2002.

[91] F. Hunt, "Origins in acoustics," Haven London, Yale Univ. Press. 1978 ..., 1978.

[92] F. Hunt, "Electroacoustics: The Analysis of Transduction, and Its Historical Background," Acoust. Soc., New York, Chap. 2, 1982. 
[93] W. Mason, "Sonics and ultrasonics: early history and applications," IEEE Trans. Sonics Ultrason., 1976.

[94] W. O. Jr, “Assessing the risks for modern diagnostic ultrasound imaging," Jpn. J. Appl. Phys., vol. 37, pa. 1, no. 5B, pp. 2781-2788, 1998.

[95] W. Smith, "The role of piezocomposites in ultrasonic transducers," Ultrason. Symp. Proceedings., IEEE, 1989.

[96] L. Smith and A. Brisken, "Front surface matched piezoelectric ultrasonic transducer array with wide field of view," US Pat. 4,211,948, 1980.

[97] J. Ketterling and O. Aristizabal, "Design and fabrication of a 40-MHz annular array transducer," IEEE Trans. Ultrason. Ferroelectr. Freq. Control, vol. 52, no. 4, pp. 672-681, 2005.

[98] T. A. Ritter, T. R. Shrout, R. Tutwiler, and K. K. Shung, “A 30-MHz piezocomposite ultrasound array for medical imaging applications," IEEE Trans. Ultrason. Ferroelectr. Freq. Control, vol. 49, no. 2, pp. 217-230, Feb. 2002.

[99] J. M. Cannata, J. A. Williams, Qifa Zhou, T. A. Ritter, and K. K. Shung, "Development of a 35-MHz piezo-composite ultrasound array for medical imaging," IEEE Trans. Ultrason. Ferroelectr. Freq. Control, vol. 53, no. 1, pp. 224-236, Jan. 2006.

[100] D. H. Turnbull, "Fabrication and Characterization of Transducer Elements in TwoDimensional Arrays for Medical Ultrasound Imaging," IEEE Trans. Ultrason. Ferroelectr. Freq. Control, vol. 39, no. 4, 1992. 
[101] A. Brisken and L. Smith, "Fabrication of front surface matched ultrasonic transducer array," US Pat. 6030299, 1979.

[102] M. Greenstein, "Electrical interconnect for multilayer transducer elements of a two-dimensional transducer array," US Pat. 8101867, 1993.

[103] G. Lockwood and C. Morton, "Ultrasound transducer array," US Pat. 6,974,417, 2005.

[104] Y. Lu and D. Horsley, "Modeling, fabrication, and characterization of piezoelectric micromachined ultrasonic transducer arrays based on cavity SOI wafers," J. Microelectromechanical Syst., vol. 24, no. 4, pp. 1142-49, 2015.

[105] Z. Wang, Q. Xue, Y. Chen, Y. Shu, H. Tian, and Y. Yang, "A flexible ultrasound transducer array with micro-machined bulk PZT," Sensors, vol. 15, no. 2, pp. $2538-47,2015$.

[106] Y. Lu, A. Heidari, S. Shelton, and A. Guedes, "High frequency piezoelectric micromachined ultrasonic transducer array for intravascular ultrasound imaging," (MEMS), IEEE 27th int. conf, Ca, USA, 2014.

[107] Y. Lu, H. Tang, S. Fung, Q. Wang, and J. Tsai, "Ultrasonic fingerprint sensor using a piezoelectric micromachined ultrasonic transducer array integrated with complementary metal oxide semiconductor electronics," Appl. Phys., vol. 106, no. 26, 2015.

[108] T. Christiansen and M. Rasmussen, "3-D imaging using row-column-addressed arrays with integrated apodization—part ii: Transducer fabrication and 
experimental results," IEEE Trans. Ultrason. Ferroelectr. Freq. Control, vol. 26, no. 5, pp. 959-71, 2015.

[109] R. A. Noble et al., "Cost-effective and manufacturable route to the fabrication of high-density 2D micromachined ultrasonic transducer arrays and (CMOS) signal conditioning electronics on the same silicon substrate," IEEE Ultrasonics Symposium. Proceedings. An International Symposium (Cat. No.01CH37263), vol. 2, pp. 941-944, 2001.

[110] "Development of piezoelectric micromachined ultrasonic transducers," Sensors Actuators A, vol. 111, no. 2-3, pp. 275-87, 2004.

[111] O. Oralkan, Xuecheng Jin, F. L. Degertekin, and B. T. Khuri-Yakub, "Simulation and experimental characterization of a 2-D capacitive micromachined ultrasonic transducer array element," IEEE Trans. Ultrason. Ferroelectr. Freq. Control, vol. 46, no. 6, pp. 1337-1340, Nov. 1999.

[112] D. Turnbull and F. Foster, "Beam steering with pulsed two-dimensional transducer arrays," IEEE Trans. Ultrason., vol. 38, no. 4, pp. 320-33, 1991.

[113] D. Turnbull and F. Foster, "Fabrication and characterization of transducer elements in two-dimensional arrays for medical ultrasound imaging," IEEE Trans. Ultrason. Ferroelectr. Freq. Control, vol. 38, no. 4, pp. 464-75, 1992.

[114] Z. Wang, W. Zhu, J. Miao, H. Zhu, and C. Chao, "Micromachined thick film piezoelectric ultrasonic transducer array," Sensors Actuators A, vol. 130-131, no. 4, pp. 485-90, 2006. 
[115] F. Akasheh, J. Fraser, and S. Bose, "Piezoelectric micromachined ultrasonic transducers: Modeling the influence of structural parameters on device performance," IEEE Trans. Ultrason. Ferroelectr. Freq. Control, vol. 52, no. 3, pp. 455-68, 2005.

[116] M. Lukacs, M. Sayer, and S. Foster, "Single element high frequency (\&lt; 50 MHz) PZT sol gel composite ultrasound transducers," IEEE Trans. Ultrason. Ferroelectr. Freq. Control, vol. 47, no. 1, pp. 148-59, 2000.

[117] D. Holmes, B. Davis, C. Bruce, and R. Robb, "3D visualization, analysis, and treatment of the prostate using trans-urethral ultrasound," Comput. Med. imaging, vol. 27, no. 5, pp. 339-49, 2003.

[118] M. Culjat, A. Dann, M. Lee, and D. Bennett, "Transurethral ultrasound catheterbased transducer with flexible polyimide joints,"IUS, 2009 IEEE int. IUS, Rome, Italy, 2009.

[119] V. Mastronardi, F. Guido, and M. Amato, "Piezoelectric ultrasonic transducer based on flexible AlN," Microelectronic, vol. 121, no. 1, pp. 59-63, 2014.

[120] X. Zhou, C. Zhao, R. Hou, J. Zhang, K. Kirk, and D. Hutson, "Sputtered ZnO film on aluminium foils for flexible ultrasonic transducers," Ultrasonics, vol. 54, no. 7, pp. 1991-8, 2014.

[121] J. Bernstein, K. Houston, L. Niles, K. Li, and H. Chen, "Integrated ferroelectric monomorph transducers for acoustic imaging," Integrated Ferroelectrics, vol. 15, no. 1-4 , pp. 289-307, 1997. 
[122] R. S. Singh et al., "Conformal Ultrasound Imaging System," Acoust. Imag, pp. 211-222, 2011.

[123] "P3D-6 Simulation, Fabrication, and Characterization of a Novel Flexible, Conformal Ultrasound Transducer Array," IEEE Symp. Ultros, NY, USA, 2007.

[124] M. Culjat, R. Singh, and C. Utley, “A flexible, conformal ultrasound array for medical imaging," Stud. Heal., 2008.

[125] O. Roy and M. Chatillon, "Ultrasonic inspection of complex geometry component specimen with a smart flexible contact phased array transducer: Modeling and application," IEEE Ultrason. Symp, Puerto Rico, USA, 2000.

[126] O. Casula, C. Poidevin, G. Cattiaux, and P. Dumas, "Control of complex components with smart flexible phased arrays," Ultrasonics, vol. 44, no. 22 , pp. e647-e651, 2006.

[127] D. Powell and G. Hayward, "Flexible ultrasonic transducer arrays for nondestructive evaluation applications. II. Performance assessment of different array configurations," IEEE Trans. Ultrason. Ferroelectr. Freq. Control, vol. 43, no. 3, pp. 393-402, 1996.

[128] D. Powell and G. Hayward, "Flexible ultrasonic transducer arrays for nondestructive evaluation applications. I. The theoretical modeling approach,” IEEE Trans. Ultrason. Ferroelectr. Freq. Control, vol. 43, no. 3, pp. 385-92, 1996.

[129] X. Zhuang, D. S. Lin, Ö. Oralkan, and B. T. Khuri-Yakub, "Fabrication of flexible 
transducer arrays with through-wafer electrical interconnects based on trench refilling with PDMS," J. Microelectromechanical Syst., vol. 17, no. 2, pp. 446452, 2008.

[130] X. Zhuang, D. Lin, and O. Oralkan, "Flexible transducer arrays with throughwafer electrical interconnects based on trench refilling with PDMS," IEEE Int Confer. MEMS, Hyogo, Japan, 2007.

[131] C. Schueler, H. Lee, and G. Wade, "Fundamentals of digital ultrasonic imaging," IEEE Trans. Sonics. Ultrason, vol. 3, no. 4, pp. 195-217,, 1984.

[132] A. Macovski, "Ultrasonic imaging using arrays," Proc. IEEE, vol. 67, no. 4, pp. 484-95, 1979.

[133] G. Kino, “Acoustic waves: devices, imaging and analog signal processing," Proc. Acoust. Sign, 1987.

[134] Q. Zhu and B. Steinberg, "Wavefront amplitude distortion and image sidelobe levels. II. In vivo experiments," IEEE Trans. Ultrason. Ferroelectr. Freq. Control, vol. 40, no. 6, pp. 754-62, 1993.

[135] V. Dutt and J. Greenleaf, "Ultrasound echo envelope analysis using a homodyned K distribution signal model," Ultrason. Imaging, vol. 16, no. 4, 1994.

[136] P. Shankar, V. Dumane, and T. George, "Classification of breast masses in ultrasonic B scans using Nakagami and K distributions," Phys. Med., vol. 48, no. $14,2003$.

[137] V. Murmis, J. Gisvold, and T. Kinter, "Texture analysis of ultrasound B-scans to 
aid diagnosis of cancerous lesions in the breast," IEEE Ultrasonics. Symp, IL, USA, 1988.

[138] S. Krass, R. Brennecke, and T. Voigtlaender, "Tissue classification by texture and spectral analysis of intracoronary ultrasound radio-frequency data," Cardiol, Vienna, Austria, 1995.

[139] F. Valckx and J. Thijssen, "Characterization of echographic image texture by cooccurrence matrix parameters," Ultrasound Med. Biol., vol. 23, no. 4, pp. 55971, 1997.

[140] A. Mojsilovic, M. Popovic, and S. Markovic, "Characterization of visually similar diffuse diseases from B-scan liver images using nonseparable wavelet transform,” IEEE Trans. Medical. Imag, vol. 17, no. 4, pp. 541-49, , 1998.

[141] H. Yoshida, D. Casalino, and B. Keserci, "Wavelet-packet-based texture analysis for differentiation between benign and malignant liver tumours in ultrasound images," Phys. Med., vol. 48, no. 22, pp. 3735-53, 2003.

[142] R. J. Lavarello Montero, "New Developments on Quantitative Imaging Using Ultrasonic Waves," University of Illinois, 2009.

[143] P. Mojabi, "Ultrasound Tomography: An Inverse Scattering Approach," University of Manitoba, 2014.

[144] W. Chew, G. Otto, W. Weedon, and J. Lin, "Nonlinear diffraction tomography-the use of inverse scattering for imaging," Imag. Sys. \& Tech, vol. 7, no. 1, pp. 16-24, 1996. 
[145] M. Moghaddam and W. Chew, "Study of some practical issues in inversion with the Born iterative method using time-domain data," IEEE Trans. Antennas, vol. 41, no. 2, pp. $177-84,1993$.

[146] P. Mojabi, M. Ostadrahimi, and L. Shafai, "Microwave tomography techniques and algorithms: A review," IEEE Xplore (ANTEM), 15 ${ }^{\text {th }}$ Int. Symp, Toulouse, France, 2012.

[147] P. Mojabi and J. LoVetri, "Comparison of TE and TM inversions in the framework of the Gauss-Newton method," IEEE Trans. Antennas, vol. 58, no. 4, pp. 1336-48, 2010.

[148] P. Mojabi and J. LoVetri, "Simultaneous reconstruction of compressibility and density profiles in multiple frequency acoustic inverse scattering," APS/URSI Symp., vol. 137, no. 4, 2013.

[149] M. Haynes and M. Moghaddam, "Large-domain, low-contrast acoustic inverse scattering for ultrasound breast imaging," IEEE Trans. Biomed., vol. 57, no. 11, pp. 2712-22, 2010.

[150] W. Chew and J. Lin, “A frequency-hopping approach for microwave imaging of large inhomogeneous bodies," IEEE Microw. Guid. Wave Lett., vol. 5, no. 12, pp. 439-41, 1995.

[151] O. Haddadin and E. Ebbini, "Imaging strongly scattering media using a multiple frequency distorted Born iterative method," IEEE Trans. Ultrason. Ferroelectr. Freq. Control, vol. 45, no. 6, pp. 1485-96, 1998. 
[152] R. Bloemenkamp and A. Abubakar, "Inversion of experimental multi-frequency data using the contrast source inversion method," Inverse, vol. 17, no. 6, pp. 161122, 2001.

[153] M. Li, O. Semerci, and A. Abubakar, "A contrast source inversion method in the wavelet domain," Inverse Probl., vol. 29, no. 2, 2013.

[154] J. Shea, P. Kosmas, and S. Hagness, "Three-dimensional microwave imaging of realistic numerical breast phantoms via a multiple-frequency inverse scattering technique," Medical Physics, vol. 37, no. 8, pp. 4210-26, 2010.

[155] W. Hu, A. Abubakar, and T. Habashy, "Simultaneous multifrequency inversion of full-waveform seismic data," Geophysics, vol. 74, no. 2, pp. R1-14, 2009.

[156] T. Mast, "Empirical relationships between acoustic parameters in human soft tissues," Acoust. Res. Lett. Online, vol. 1, no. 2, 2000.

[157] A. Hesford and R. Waag, "Reduced-rank approximations to the far-field transform in the gridded fast multipole method," J. Comput. Phys., vol. 230, no. 10, pp. 3656-67, 2011.

[158] M. Moghaddam and W. Chew, "Nonlinear two-dimensional velocity profile inversion using time domain data," IEEE Trans. Geosci., vol. 30, no. 1, pp. $147-$ $56,1992$.

[159] J. Hadamard, Lectures on Cauchy's problem in linear partial differential equations. 2014.

[160] P. Huthwaite, F. Simonetti, and N. Duric, "Combining time of flight and 
diffraction tomography for high resolution breast imaging: initial invivo results (1)," Acoust. Soc. Am., vol. 132, no. 3, 2012.

[161] C. Li, N. Duric, and L. Huang, "Comparison of ultrasound attenuation tomography methods for breast imaging," Med. Imaging, vol. 6920, CA, USA, 2008.

[162] P. Huthwaite and F. Simonetti, "High-resolution imaging without iteration: A fast and robust method for breast ultrasound tomography," J. Acoust. Soc., vol. 130, no. 3, 2011.

[163] C. Li, N. Duric, P. Littrup, and L. Huang, "In vivo breast sound-speed imaging with ultrasound tomography," Ultrasound Med. Biol., vol. 35, no. 10, pp. 1615-28, 2009.

[164] J. Wiskin, D. Borup, and S. Johnson, "Non-linear inverse scattering: High resolution quantitative breast tissue tomography," J. Acoust. Soc., vol. 131, no. 5, 2012.

[165] F. Kamangar, G. Dores, and W. Anderson, "Patterns of cancer incidence, mortality, and prevalence across five continents: defining priorities to reduce cancer disparities in different geographic regions of the," J. Clin. Oncol., vol. 24, pp. 2137-50, 2006.

[166] A. Dhawan, Medical Image Analysis. New Jersey: Wiley Inter Science, 2003.

[167] M. Kriege, C. Brekelmans, and C. Boetes, "Efficacy of MRI and mammography for breast-cancer screening in women with a familial or genetic predisposition," Engl. J. Medicine, 2004. 
[168] F. Simonetti, L. Huang, and N. Duric, “A multiscale approach to diffraction tomography of complex three-dimensional objects," Appl. Phys. Lett., vol. 95, no. $6,2009$.

[169] G. Faucher, Calibration of an ultrasound tomography system for medical imaging with 2D contrast-source inversion, University of Manitoba, 2013.

[170] T. Szabo, Diagnostic ultrasound imaging: inside out, Elsevier, 2004.

[171] M. Slaney, A. Kak, and L. Larsen, "Limitations of imaging with first-order diffraction tomography," IEEE Trans. Microw, vol. 32, no. 8, pp. 860-74, 1984.

[172] R. Pierri, G. Leone, and R. Persico, "Second-order iterative approach to inverse scattering: numerical results," JOSA A, vol. 17, no. 5, pp. 874-80, 2000.

[173] A. Kak and M. Slaney, Principles of computerized tomographic imaging. 2001.

[174] N. Duric, P. Littrup, L. Poulo, and A. Babkin, "Detection of breast cancer with ultrasound tomography: First results with the Computed Ultrasound Risk Evaluation (CURE) prototype," Medical Physics, vol. 34, no. 2, pp. 773-85, 2007.

[175] A. Hormati, I. Jovanovic, O. Roy, and M. Vetterli, "Robust ultrasound travel-time tomography using the bent ray model," Proc. SPIE, vol. 7629, San Diego, CA, 2010.

[176] R. Steinhausen, "Modelling and Characterization of Piezoelectric 1-3 Fiber Composites,"pp. 199-204, 2011.

[177] "1-3 Random Fiber Composites.” [Online]. Available: http://www.smartmaterial.com/13Crand-product-main.html. [Accessed: 17-May-2017]. 
[178] T. Furuhata, T. Hirano, and H. Fujita, "Array-driven ultrasonic microactuators," IEEE Int. Conf. Solid. State. Sensor, San Francisco, CA, 1991.

[179] C. R. Hill, J. C. Bamber, and G. Haar, Physical principles of medical ultrasonics. John Wiley \& Sons, 2004.

[180] K. Zell, J. I. Sperl, M. W. Vogel, R. Niessner, and C. Haisch, “Acoustical properties of selected tissue phantom materials for ultrasound imaging," Phys. Med. Biol., vol. 52, no. 20, pp. N475-N484, Oct. 2007.

[181] S. L. James, "Transcranial Ultrasound as a Potential Modality for Real-Time Observation of Brain Motion.” Cleveland State University, 2017.

[182] G. Clement and K. Hynynen, "Field characterization of therapeutic ultrasound phased arrays through forward and backward planar projection," J. Acoust. Soc., vol. 108, no. 1, 2000.

[183] K. Hynynen, "The role of nonlinear ultrasound propagation during hyperthermia treatments," Med. Phys., vol. 18, no. 6, pp. 1156-63, 1991.

[184] "FI Toolbox." [Online]. Available: http://www.diagnosticsonar.com/fittext.html. [Accessed: 23-Jun-2017].

[185] "FlexRIO Custom Instruments and Processing - Electronic Test and Instrumentation - National Instruments.” [Online]. Available: http://www.ni.com/en-us/shop/select/flexrio-custom-instruments-and-processingcategory?storeId=10201\&userLocale $=$ enUS\&requestLanguage =en\&localeGroup=en-us\&requestLangId=-1\&langId=-1 . 
[Accessed: 23-Jun-2017].

[186] "China PCB Prototype \&amp; Fabrication Manufacturer - PCB Prototype the Easy Way.” [Online]. Available: https://www.pcbway.com/. [Accessed: 18-Jul-2017].

[187] “3ders.org - The Best 3D Scanners of 2015 |3D Printer News \&amp; 3D Printing News." [Online]. Available: http://www.3ders.org/articles/20151209-best-3dscanners-2015.html. [Accessed: 03-Oct-2017]. 
APPENDIX 


\section{APPENDIX A}

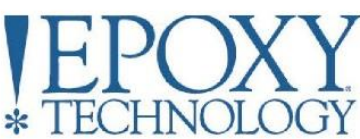

\section{EPO-TEK ${ }^{\circledR}$ E4110 \\ Technical Data Sheet \\ For Reference Only \\ Electrically Conductive, Silver Epoxy}

\section{EPO-TEK ${ }^{\circledR}$ E4110 Advantages \& Suggested Application Notes:}

- Ease of use: smooth flowing paste allows for automated dispensing, stamping, brushing or hand applications. In some cases, the low viscosity nature of the paste allows it to be sprayed onto targets.

- Suggested applications include: EMI and Rf shielding, ITO interconnects in LCDs, low temperature cryogenic cooling.

- Exhibits superior adhesion to a wide variety of substrates including most metals, ceramics glass and plastics.

- Hybrid / Micro-electronic adhesive including die-attach and substrate attach for Rf and Microwave devices.

- Bright and shiny silver epoxy; provides a metallic-like layer after cure.

Epoxies and Adhesives for Demanding Applications ${ }^{\mathrm{TM}}$

This information is based on data and tests believed to be accurate. Epoxy Technology, Inc. makes no warranties (expressed or implied) as to its accuracy and assumes no liability in connection with any use of this product.

14 FORTUNE DRIVE, BILLERICA, MA 01821 (978) 667-3805, FAX (978) 663-9782 www.epotek.com 


\section{IEPOXY \\ * TECHNOLOGY}

EPO-TEK ${ }^{\circledR}$ E4110

Technical Data Sheet

For Reference Only

Electrically Conductive, Silver Epoxy

Recommended Cure: $150^{\circ} \mathrm{C} / 1$ Hour

Minimum Alternative Cure(s):

May not achieve performance properties listed below

$100^{\circ} \mathrm{C} / 1$ Hour

$60^{\circ} \mathrm{C} / 6$ Hours

$23^{\circ} \mathrm{C} / 3$ Days

NOTES

Container(s) should be kept closed when not in use

Filed systems should be stirred thoroughly before mixing and prior to use

列 delivered status/container into any other containers of any kind, including but not limited to syringes, bi-paks, cartridges, pouches, tubes, capsules, fims or other packages.

- Syringe packaging will impact initial viscosity and effective pot life, potentially beyond stated parameters.

Product Description: EPO-TEK@ E4110 is an electrically conductive, silver-filled epoxy paste. This two component system is

designed for low temperature curing from ambient to $80^{\circ} \mathrm{C}$, although other heat cures can be used.

Typical Properties: Cure condition: $150^{\circ} \mathrm{C} / 1$ Hour Different batches, conditions \& applications yield differing results Data below is not guaranteed. To be used as a guide only, not as a specification. * denotes test on lot acceptance basis

\begin{tabular}{|c|c|c|}
\hline PHYSICAL PROPERTIES: & & \\
\hline * Color (before cure): & Part A: Silver & it B: Clear \\
\hline * Consistency: & Smooth flowing past & \\
\hline *Viscosity $\left(23^{\circ} \mathrm{C}\right) @ 100 \mathrm{rpm}:$ & $800-1,600$ & cPs \\
\hline Thixotropic Index: & 2.1 & \\
\hline * Glass Transition Temp: & $\geq 40$ & ${ }^{\circ} \mathrm{C}$ (Dynamic Cure: 20-200 $0^{\circ} \mathrm{C} / \mathrm{SO} 25 \mathrm{Min}$; Ramp -10-200 $920^{\circ} \mathrm{C} / \mathrm{Min}$ ) \\
\hline Coefficient of Thermal Expansion (CTE): & & \\
\hline Below Tg: & 48 & $\times 10^{-8} \mathrm{in} / \mathrm{in}^{\circ} \mathrm{C}$ \\
\hline Shore $\mathrm{D}$ Hardness. & 150 & $\times 10^{-6} \mathrm{in} / \mathrm{in}^{\circ} \mathrm{C}$ \\
\hline Shore D Hardness: & 60 & \\
\hline 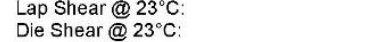 & $\begin{array}{r}1,266 \\
\geq 5\end{array}$ & $1,778 \mathrm{psi}$ \\
\hline Degradation Temp: & 380 & ${ }^{\circ} \mathrm{C}$ \\
\hline @200 2 : & 0.70 & $\%$ \\
\hline Suggested Operating Temperature: & $<250$ & ${ }^{\circ} \mathrm{C}$ (Intermittent) \\
\hline Storage Modulus: & 518,756 & psi \\
\hline Ion Content: & $151 \mathrm{ppm}$ & $23 \mathrm{ppm}$ \\
\hline * Particle Size: & $23 \mathrm{ppm}$ & $31 \mathrm{ppm}$ \\
\hline
\end{tabular}

ELECTRICAL AND THERMAL PROPERTIES:

* Thermal Conductivity: $23^{\circ} \mathrm{C}\left(150^{\circ} \mathrm{C} / 1\right.$ Hour):

$1.4 \mathrm{~W} / \mathrm{mK}$

$\leq 0.0005 \quad$ Ohm-cm

* Volume Resistivity $23^{\circ} \mathrm{C}\left(25^{\circ} \mathrm{C} 40-60 \% \mathrm{RH} / 3\right.$ Day Cure $): \leq 0.009$ Ohm- $\mathrm{cm}$

Epoxies and Adhesives for Demanding Applications
This information is based on data and tests believed to be accurate. Epoxy Technology, Inc. makes no warranties (expressed or implied) as to its accuracy and assumes no liability in connection with any use of this product.

EPOXY TECHNOLOGY, INC.

14 FORTUNE DRIVE, BILLERICA, MA 01821 (978) 667-3805, FAX (978) 663-9782

www.epotek.com 


\section{APPENDIX B}

\section{LOCTITE}

Technical Data Sheet

\section{LOCTITE ${ }^{\circledR} 493$}

November 2010

PRODUCT DESCRIPTION
LOCTITE 493 provides the following product characteristics:
\begin{tabular}{|l|l|}
\hline Technology & Cyanoacrylate \\
\hline Chemical Type & Methyl cyanoacrylate \\
\hline Appearance (uncured) & $\begin{array}{l}\text { Clear to slightly hazy, colorless liquid } \\
\text { wes }\end{array}$ \\
\hline Components & One part - requires no mixing \\
\hline Viscosity & Very low \\
\hline Cure & Humidity \\
\hline Application & Bonding \\
\hline Key Substrates & Metals, Rubbers and Plastics \\
\hline
\end{tabular}

LOCTITE $^{2} 493$ is a general purpose adhesive and is particularly suited to bonding of metal substrates.

Commercial Item Description A-A-3097:

LOCTITE $^{2} 493$ has been qualified to Commercial Item Description A-A-3097. Note: This is a regional approval. Please contact your local Technical Service Center for more information and clarification.

\section{TYPICAL PROPERTIES OF UNCURED MATERIAL \\ Specific Gravity @ $25^{\circ} \mathrm{C}$ \\ Viscosity, Cone \& Plate, $\mathrm{mPa} \cdot \mathrm{s}(\mathrm{cP})$ : \\ Temperature: $25^{\circ} \mathrm{C}$, Shear Rate: $3,000 \mathrm{~s}^{-4}$ \\ Viscosity, Brookfield - LVF, $25^{\circ} \mathrm{C}, \mathrm{mPa} \cdot \mathrm{s}$ (cP): \\ Spindle 1, speed $60 \mathrm{rpm}$, \\ Vapour Pressure, hPa \\ Flash Point - See SDS \\ 1.1 \\ 1 to $4^{\text {uns }}$ \\ 1 to 10 \\ $<1$}

\section{TYPICAL CURING PERFORMANCE}

Under normal conditions, the atmospheric moisture initiates the curing process. Although full functional strength is developed in a relatively short time, curing continues for at least 24 hours before full chemical/solvent resistance is developed.

\section{Cure Speed vs. Substrate}

The rate of cure will depend on the substrate used. The table below shows the fixture time achieved on different materials at $22{ }^{\circ} \mathrm{C} / 50 \%$ relative humidity. This is defined as the time to develop a shear strength of $0.1 \mathrm{~N} / \mathrm{mm}^{2}$.

Fixture Time, seconds:

Mild Steel (degreased) 20 to 40

Aluminum (degreased)

Zinc dichromate $\quad 30$ to 60

Neoprene $\quad<10$

Rubber, nitrile $\quad<10$

ABS 10 to 30

PVC $\quad 30$ to 70

Polycarbonate 20 to 70

Phenolic 10 to 30

Cure Speed vs. Bond Gap

The rate of cure will depend on the bondline gap. Thin bond lines result in high cure speeds, increasing the bond gap will decrease the rate of cure.

Cure Speed vs. Humidity

The rate of cure will depend on the ambient relative humidity. The following graph shows the tensile strength developed with time on Buna $\mathrm{N}$ rubber at different levels of humidity.

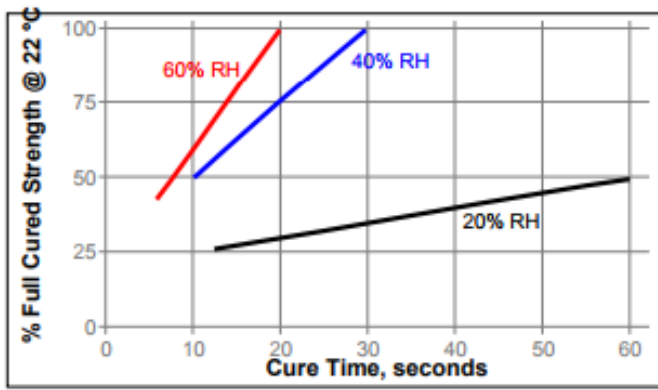

Cure Speed vs. Activator

Where cure speed is unacceptably long due to large gaps, applying activator to the surface will improve cure speed. However, this can reduce ultimate strength of the bond and therefore testing is recommended to confirm effect.

\section{TYPICAL PROPERTIES OF CURED MATERIAL}

\section{After 24 hours @ $22^{\circ} \mathrm{C}$}

Physical Properties:

Coefficient of Thermal Expansion, $\quad 100 \times 10^{\circ}$

ISO 11359-2, K

Coefficient of Thermal Conductivity, ISO $8302, \quad 0.1$ W/(m-K)

Electrical Properties:

Dielectric Constant / Dissipation Factor, IEC 60250:

$\begin{array}{ll}0.1 \mathrm{kHz} & 2 \text { to } 3.3 /<0.02 \\ 1 \mathrm{kHz} & 2 \text { to } 3.5 /<0.02\end{array}$

$10 \mathrm{kHz} \quad 2$ to $3.5 /<0.02$

Volume Resistivity, IEC $60093, \Omega \cdot \mathrm{cm} \quad 2 \times 10^{15}$ to $10 \times 10^{15}$ Surface Resistivity, IEC $60093, \Omega \quad 10 \times 10^{15}$ to $80 \times 10^{15}$ Dielectric Breakdown Strength,

IEC 60243-1, kV/mm
25 
TDS LOCTITE ${ }^{\circledR} 493$, November 2010

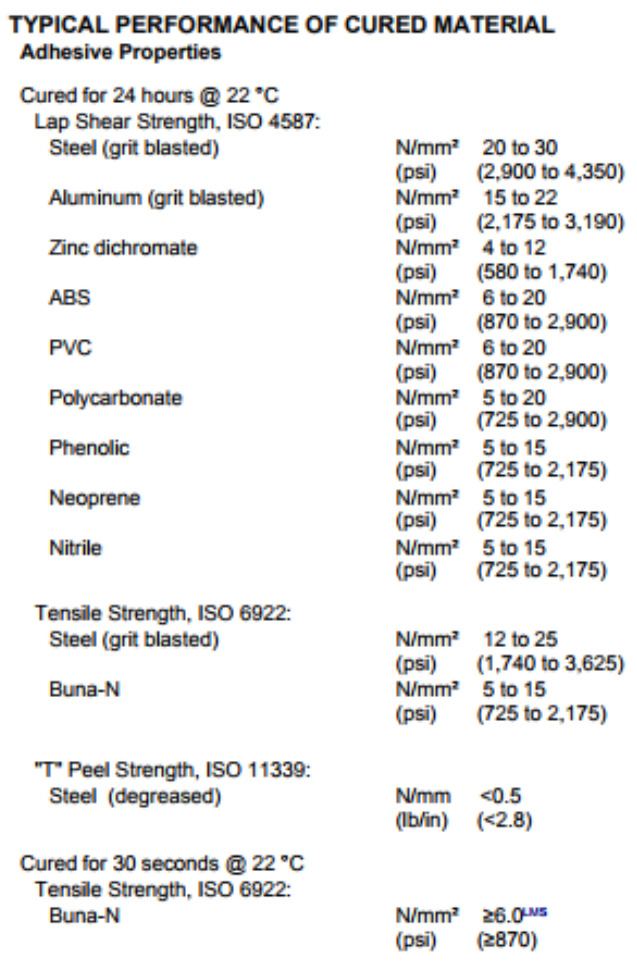

TYPICAL ENVIRONMENTAL RESISTANCE

After 1 week @ $22^{\circ} \mathrm{C}$

Lap Shear Strength, ISO 4587:

Mild steel (grit blasted)

\section{Hot Strength}

Tested at temperature

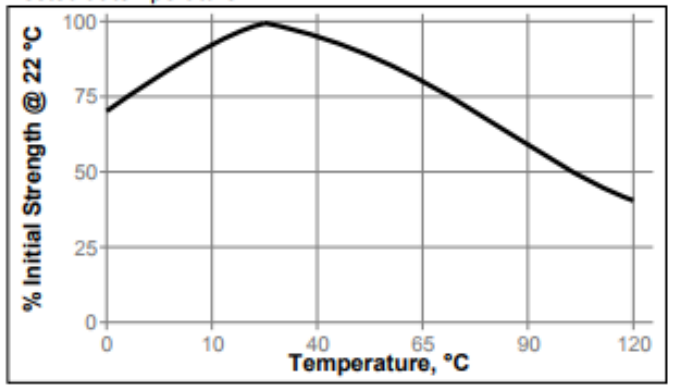

Heat Aging

Aged at temperature indicated and tested @ $22^{\circ} \mathrm{C}$

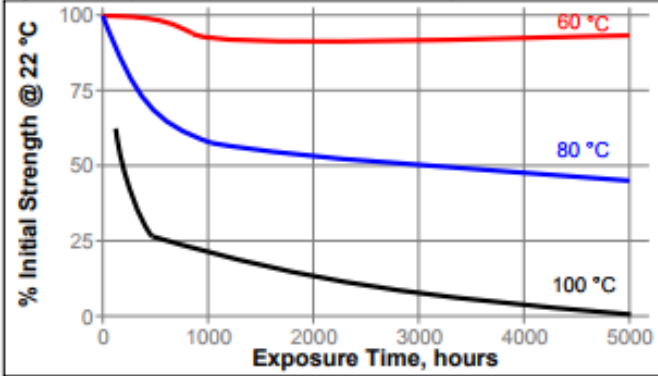

Chemical/Solvent Resistance

Aged under conditions indicated and tested @ $22^{\circ} \mathrm{C}$.

\begin{tabular}{|l|c|c|c|c|}
\hline & & \multicolumn{3}{|c|}{$\%$ of initial strength } \\
\hline Environment & ${ }^{\circ} \mathbf{C}$ & $\mathbf{1 0 0 ~} \mathbf{h}$ & $\mathbf{5 0 0} \mathbf{~ h}$ & $\mathbf{1 0 0 0} \mathbf{~}$ \\
\hline Motor oil (MIL-L-46152) & 40 & 100 & 100 & 100 \\
\hline Gasoline & 22 & 95 & 95 & 95 \\
\hline Isopropanol & 22 & 95 & 95 & 95 \\
\hline Ethanol & 22 & 100 & 100 & 100 \\
\hline Freon TA & 22 & 95 & 95 & 95 \\
\hline $1,1,1$ Trichloroethane & 22 & 95 & 95 & 95 \\
\hline Heat/humidity 95\% RH & 40 & 70 & 50 & 40 \\
\hline $\begin{array}{l}\text { Heat/humidity 95\% RH } \\
\text { on polycarbonate }\end{array}$ & 40 & 95 & 95 & 95 \\
\hline
\end{tabular}

\section{GENERAL INFORMATION}

This product is not recommended for use in pure oxygen and/or oxygen rich systems and should not be selected as a sealant for chlorine or other strong oxidizing materials.

For safe handling information on this product, consult the Safety Data Sheet (SDS).

Directions for use:

1. For best performance bond surfaces should be clean and free from grease.

2. This product performs best in thin bond gaps $(0.05 \mathrm{~mm})$.

3. Excess adhesive can be dissolved with Loctite cleanup solvents, nitromethane or acetone.

Loctite Material Specification wes

LMS dated June 06, 2002. Test reports for each batch are available for the indicated properties. LMS test reports include selected QC test parameters considered appropriate to specifications for customer use. Additionally, comprehensive controls are in place to assure product quality and consistency. Special customer specification requirements may be coordinated through Henkel Quality. 
Storage

Store product in the unopened container in a dry location. Storage information may be indicated on the product container labeling.

Optimal Storage: $2{ }^{\circ} \mathrm{C}$ to $8{ }^{\circ} \mathrm{C}$. Storage below $2{ }^{\circ} \mathrm{C}$ or greater than $8{ }^{\circ} \mathrm{C}$ can adversely affect product properties. Material removed from containers may be contaminated during use. Do not retum product to the original container. Henkel Corporation cannot assume responsibility for product which has been contaminated or stored under conditions other than those previously indicated. If additional information is required, please contact your local Technical Service Center or Customer Service Representative.

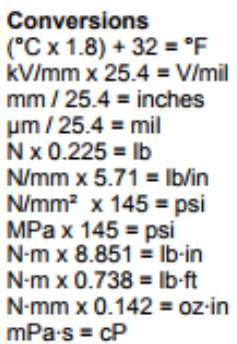

Note:

The information provided in this Technical Data Sheet (TDS) including the recommentations for use and application of the product are based on our knowledge and experience of the product as at the date of this TDS. The product can have a variety of different applications as well as TDS. The product can have a variety of different applications as well as differing application and working conditions in your environment that of our product for the production prose, nond conditions in respect of which you use them, as well as the intended applications and results. We strongly recommend that you carry out your own prior trials to confirm such suitability of our product.

Any liability in respect of the information in the Technical Data Sheet or any other written or oral recommendation(s) regarding the concerned product is excluded, except if otherwise explicitly agreed and except in relation to death or personal injury caused by our negligence and any relation to death or personal injury caused by our negligen
liability under any applicable mandatory product liability law.

In case products are delivered by Henkel Belgium NV, Henkel Electronic Materials NV, Henkel Nederland BV, Henkel Technologies France SAS and Henkel France SA please additionally note the following:

In case Henkel would be nevertheless held liable, on whatever legal ground, Henkel's liability will in no event exceed the amount of the concerned delivery.

In case products are delivered by Henkel Colombiana, S.A.S. the

following disclaimer is applicable: The information provided in this Technical Data Sheet (TDS) including the recommendations for use and application of the product are based on our knowledge and experience of the product as at the date of this TDS. Henkel is, therefore, not liable for the suitability of our product for the production processes and conditions in respect of which you use them, as well as the intended applications and results. We strongly recommend that you carry out your own prior trials to confirm such suitability of our product.

Any liability in respect of the information in the Technical Data Sheet or any other written or oral recommendation(s) regarding the concerned product is excluded, except if otherwise explicitly agreed and except in relation to death or personal injury caused by our negligence and any liability under any applicable mandatory product liability law.

In case products are delivered by Henkel Corporation, Resin Technology Group, Inc., or Henkel Canada Corporation, the following disclaimer is applicable:

The data contained herein are furnished for information only and are believed to be reliable. We cannot assume responsibility for the results obtained by others over whose methods we have no control. It is the user's responsibility to determine suitability for the user's purpose of any production methods mentioned herein and to adopt such any production methods mentioned herein and to adopt such precautions as may be advisable for the protection of property and of
persons against any hazards that may be involved in the handling and persons against any hazards that may be involved in the handling and
use thereof. In light of the foregoing, Henkel Corporation specifically disclaims all warranties expressed or implied, including warranties of merchantability or fitness for a particular purpose, arising from sale or use of Henkel Corporation's products. Henkel Corporation specifically disclaims any liability for consequential or incidental damages of any kind, including lost profits. The discussion herein of various processes or compositions is not to be interpreted as representation that they are free from domination of patents ourned by others or as a license under any Henkel Corporation patents owned by others or as a license under any Henkel Corporation patents that may cover such processes or compositions. We recommend that each prospective user test his proposed application before repetitive use, using this data as a guide. This product may be covered by one or more United States or foreign patents or patent applications.

Trademark usage

Except as otherwise noted, all trademarks in this document are trademarks of Henkel Corporation in the U.S. and elsewhere. denotes a trademark registered in the U.S. Patent and Trademark Office.

Reference 1.3

$\begin{array}{lcr}\text { Henkel Americas } & \text { Henkel Europe } & \text { Henkel Asia Pacific } \\ \mathbf{+ 8 6 0 . 5 7 1 . 5 1 0 0} & +49.89 .320800 .1800 & +86.21 .2891 .8859\end{array}$

For the most direct access to local sales and technical support visit: www.henkel.com/industrial 


\section{APPENDIX C}

\section{MAX14752/MAX14753}

\section{8-Channel/Dual 4-Channel 72V Analog Multiplexers}

\section{General Description}

The MAX14752/MAX14753 are 8-to-1 and dual 4-to-1 high-voltage analog multiplexers. Both devices feature $60 \Omega$ (typ) on-resistance with $0.03 \Omega$ (typ) on-resistance flatness. These low on-resistance multiplexers conduct equally well in either direction. Flexible logic levels for the channel-select interface are defined by the EN input. The MAX14752 is a 8-to-1 multiplexer and MAX14753 is a dual 4-to-1 multiplexer. Both devices operate with dual supplies of $\pm 10 \mathrm{~V}$ to $\pm 36 \mathrm{~V}$, or a single supply of $+20 \mathrm{~V}$ to $+72 \mathrm{~V}$.

The MAX14752/MAX14753 are available in a 16-pin TSSOP package and are pin compatible with the industry-standard DG408/DG409. Both the MAX14752/ MAX14753 are specified over the extended $-40^{\circ} \mathrm{C}$ to $+85^{\circ} \mathrm{C}$ operating temperature range.

\section{Applications}

Programmable-Logic Controllers

Environment Control Systems

ATE Systems

Medical Monitoring Systems

\section{Benefits and Features}

- Supply Range Accommodates Wide Voltage Applications

- Wide $\pm 36 \mathrm{~V}$ (max) Bipolar Power Supply

- High +72V (max) Single Power Supply

- Asymmetric Bipolar Power Supply Operation

- Low Leakage Current and RON Improves System Accuracy

- Low On-Resistance $60 \Omega$ (typ)

- $0.03 \Omega$ (typ) RoN Flatness Over Common-Mode Voltage Range

- 20nA Low-Input, On-Leakage Current (max)

- Low 25HA IDD Supply Current in Disable Mode Saves Power

- Easy to Upgrade with Pin-Compatible, IndustryStandard DG408/DG409

\section{Ordering Information}

\begin{tabular}{|ccl|}
\hline PART & TEMP RANGE & PIN-PACKAGE \\
\hline MAX14752EUE + & $-40^{\circ} \mathrm{C}$ to $+85^{\circ} \mathrm{C}$ & 16 TSSOP \\
\hline MAX14753EUE + & $-40^{\circ} \mathrm{C}$ to $+85^{\circ} \mathrm{C}$ & 16 TSSOP \\
\hline
\end{tabular}

+Denotes a lead(Pb)-free/RoHS-compliant package.

Pin Configurations appear at end of data sheet.

Functional Diagrams

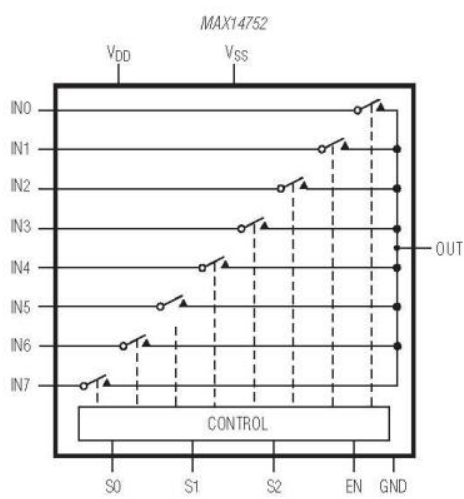

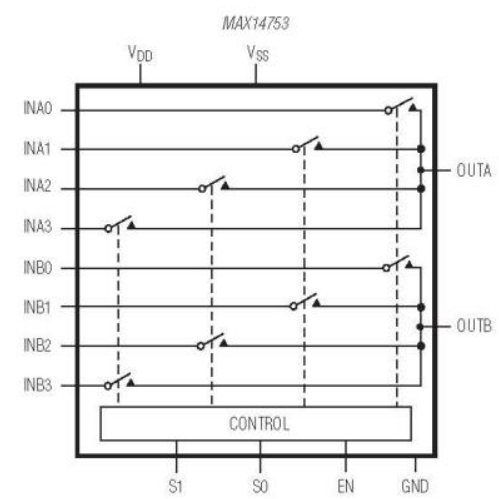




\section{Absolute Maximum Ratings}

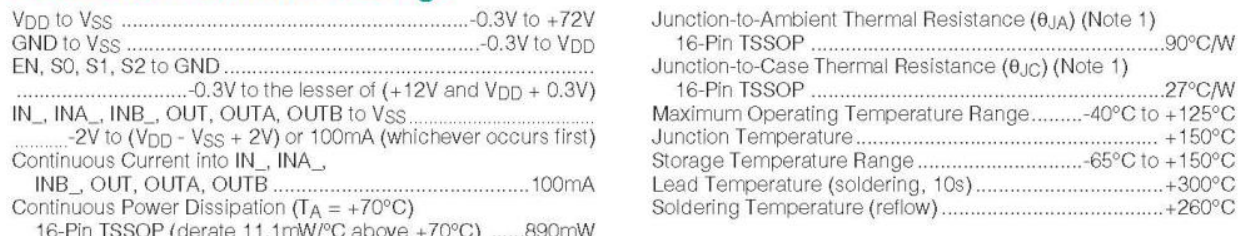

16-Pin TSSOP (derate $11.1 \mathrm{~mW} /{ }^{\circ} \mathrm{C}$ above $+70^{\circ} \mathrm{C}$ ) $\ldots . . .890 \mathrm{~mW}$

Note 1: Package thermal resistances were obtained using the method described in JEDEC specification JESD51-7, using a four-layer board. For detailed information on package thermal considerations, refer to www.maximintegrated.com/thermal-tutorial.

Stresses beyond those listed under "Absolute Maximum Ratings" may cause permanent damage to the device. These are stress ratings only, and functional operation of the device at these or any other conditions beyond those indicated in the operational sections of the specifications is not implied. Exposure to absolute maximum rating conditions for extended periods may affect device relliability.

DC Electrical Characteristics-Dual Supplies

$\left(V_{D D}=+35 V, V_{S S}=-35 V, V_{G N D}=0 V, V_{E N}=+3.3 V, T_{A}=-40^{\circ} \mathrm{C}\right.$ to $+85^{\circ} \mathrm{C}$, unless otherwise noted. Typical values are at $\left.T_{A}=+25^{\circ} \mathrm{C}.\right)$

\begin{tabular}{|c|c|c|c|c|c|c|}
\hline PARAMETER & SYMBOL & CONDITIONS & MIN & TYP & MAX & UNITS \\
\hline \multicolumn{7}{|l|}{ POWER SUPPLY } \\
\hline VDD Supply Voltage Range & $V_{D D}$ & & +10 & & +36 & $\mathrm{~V}$ \\
\hline VSS Supply Voltage Range & $V_{S S}$ & & -10 & & -36 & $\mathrm{~V}$ \\
\hline \multirow[b]{2}{*}{$V_{D D}$ Supply Current } & $\mathrm{IDD}(\mathrm{OFF})$ & $V_{E N}=V_{S_{-}}=O V, V_{I N}=V_{I N A_{-}}=V_{\mid N B_{-}}=+20 \mathrm{~V}$ & & 12 & 25 & \multirow[b]{2}{*}{$\mu \mathrm{A}$} \\
\hline & IDD(ON) & $\begin{array}{l}V_{E N}=+5 V, V_{S_{-}}=0 \mathrm{~V} \text { or } V_{E N}, V_{I N}=V_{I N A}= \\
V_{I N B_{-}}=+20 \mathrm{~V}\end{array}$ & & 270 & 600 & \\
\hline \multirow[b]{2}{*}{ VSS Supply Current } & ISS(OFF) & $V_{E N}=V_{S_{-}}=O V, V_{I N}=V_{I N A_{-}}=V_{\mid N B_{-}}=+20 \mathrm{~V}$ & & 11 & 25 & \multirow[b]{2}{*}{$\mu \mathrm{A}$} \\
\hline & ISS(ON) & $\begin{array}{l}V_{E N}=+5 V, V_{S_{-}}=0 \mathrm{~V} \text { or } V_{E N}, V_{I N}=V_{I N A}= \\
V_{I N B_{-}}=+20 \mathrm{~V}\end{array}$ & & 260 & 600 & \\
\hline \multicolumn{7}{|l|}{ ANALOG MUX } \\
\hline Analog Signal Range & $\begin{array}{l}\text { VIN_, VINA_, } \\
\text { VINB, VOUT, } \\
\text { VOUTA, VOUTB } \\
\end{array}$ & & VSS & & VDD & V \\
\hline Current Through Multiplexer & 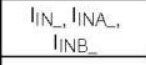 & $V_{I N}, V_{I N A}, V_{\mid N B_{-}}= \pm 20 \mathrm{~V}$ & -5 & & +5 & $\mathrm{~mA}$ \\
\hline On-Resistance & RON & $\begin{array}{l}\text { IIN_, IINA_, IINB }=5 \mathrm{~mA} ; \text { VIN, VINA, VINB, } \\
\text { VOUT, VOUTA, VOUTB }= \pm 20 \mathrm{~V} \text {, Figure } 1 \\
\end{array}$ & & 60 & 130 & $\Omega$ \\
\hline $\begin{array}{l}\text { On-Resistance Matching } \\
\text { Between Channels }\end{array}$ & $\Delta R_{O N}$ & $\begin{array}{l}I_{\mid N_{-},} I_{I N A_{-}}, I_{\mid N B_{-}}=5 \mathrm{~mA}, V_{I} N_{-}, V_{I N A}, V_{I N B_{-}}= \\
\pm 20 \mathrm{~V}, 0 \mathrm{~V}\end{array}$ & \multicolumn{3}{|c|}{0.5} & $\Omega$ \\
\hline On-Resistance Flatness & RFLAT_(ON) & $\begin{array}{l}\text { IIN_IINA_, IINB }=5 m A, V \mid N_{-}, V_{I N A}, V_{I N B} \\
\text { VOUT, VOUTA, VOUTB }= \pm 2 O \mathrm{~V}\end{array}$ & \multicolumn{3}{|c|}{0.03} & $\Omega$ \\
\hline \multirow{2}{*}{ Output On-Leakage Current } & \multirow{2}{*}{ IOUT(ON) } & $\begin{array}{l}\text { MAX14752: VOUT, VOUTA, VOUTB }= \pm 20 \mathrm{~V}, \\
\text { VIN_, VINA_, VINB_ = unconnected, Figure } 2\end{array}$ & -20 & & +20 & \multirow{2}{*}{$n A$} \\
\hline & & $\begin{array}{l}\text { MAX14753: VOUT, VOUTA, VOUTB }= \pm 20 \mathrm{~V} \\
V_{\mid N}, V_{\mid N A}, V_{\mid N B}=\text { unconnected, Figure } 2\end{array}$ & -10 & & +10 & \\
\hline
\end{tabular}


MAX14752/MAX14753

8-Channel/Dual 4-Channel $72 \mathrm{~V}$ Analog Multiplexers

DC Electrical Characteristics-Dual Supplies (continued)

$\left(V_{D D}=+35 \mathrm{~V}, V_{S S}=-35 \mathrm{~V}, V_{G N D}=\mathrm{OV}, V_{E N}=+3.3 \mathrm{~V}, \mathrm{~T}_{\mathrm{A}}=-40^{\circ} \mathrm{C}\right.$ to $+85^{\circ} \mathrm{C}$, unless otherwise noted. Typical values are at $\mathrm{T}_{\mathrm{A}}=+25^{\circ} \mathrm{C}$.

\begin{tabular}{|c|c|c|c|c|c|c|}
\hline PARAMETER & SYMBOL & CONDITIONS & MIN & TYP & MAX & UNITS \\
\hline \multirow{2}{*}{ Output Off-Leakage Current } & \multirow{2}{*}{ IOUT(OFF) } & 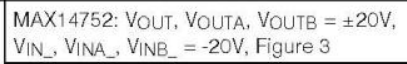 & -20 & & +20 & \multirow{2}{*}{$\mathrm{nA}$} \\
\hline & & $\begin{array}{l}\text { MAX14753: VOUT, VOUTA, VOUTB }= \pm 40 \mathrm{~V}, \\
V_{I N \_}, V_{I N A}, V_{I N B}=-40 \mathrm{~V} \text {, Figure } 3\end{array}$ & -10 & & +10 & \\
\hline Input Off-Leakage Current & IIN(OFF) & $\begin{array}{l}\text { VOUT, VOUTA, VOUTB }= \pm 20 \mathrm{~V}, V_{I N}, V_{I N A}, \\
V_{I N B_{-}}= \pm 20 \mathrm{~V}, \text { Figure } 3\end{array}$ & -5 & & +5 & $n A$ \\
\hline \multicolumn{7}{|l|}{ LOGIC (EN, S0, S1, S2) } \\
\hline EN Input Voltage Low & VEN_IL & & & & 0.8 & V \\
\hline EN Input Voltage High & VEN_IH & & 2.1 & & & $\mathrm{~V}$ \\
\hline EN, S_Input Voltage Range & $V_{E N}, V_{S_{-}}$ & & & & 11 & $\mathrm{~V}$ \\
\hline EN Input Current & IEN_IH(DC) & $\begin{array}{l}V_{E N}=+11 V, V_{S O}=V_{S 1}=V_{S 2}=\left(0.25 \times V_{E N}\right) \\
\text { or }\left(0.75 \times V_{E N}\right)\end{array}$ & & & 0.4 & $\mathrm{~mA}$ \\
\hline So, S1, S2 Input Voltage Low & $V_{I L}$ & & & & $\begin{array}{l}0.25 x \\
V_{E N}\end{array}$ & V \\
\hline So, S1, S2 Input Voltage High & $V_{I H}$ & & $\begin{array}{c}0.75 x \\
\text { VEN } \\
\end{array}$ & & & V \\
\hline \multicolumn{7}{|l|}{ DYNAMIC CHARACTERISTICS } \\
\hline Enable Turn-On Time & ton & $V_{\mid N O}, V_{I N A O}= \pm 10 \mathrm{~V}, R_{L}=10 \mathrm{k} \Omega$, Figure 4 & & 1 & 25 & $\mu \mathrm{s}$ \\
\hline Enable Turn-Off Time & tOFF & $\mathrm{V}_{\mid N \mathrm{NO}}, \mathrm{V}_{\mid N A O}= \pm 10 \mathrm{~V}, \mathrm{RL}_{\mathrm{L}}=10 \mathrm{k} \Omega$, Figure 4 & & 0.8 & 2 & $\mu \mathrm{s}$ \\
\hline Transition Time & ITRANS & $V_{\mid N O}, V_{\mid N A O}= \pm 10 \mathrm{~V}, R_{L}=10 \mathrm{k} \Omega$, Figure 5 & & 10 & & $\mu s$ \\
\hline Break-Before-Make Time Delay & tBBM & $\begin{array}{l}V_{I N}, V_{I N A}, V_{I N B_{-}}= \pm 10 \mathrm{~V}, R_{L}=10 \mathrm{k} \Omega \\
\text { Figure } 6\end{array}$ & & 10 & & $\mu s$ \\
\hline Frequency Response & BW & $\mathrm{P}_{S}=50 \Omega, \mathrm{RL}_{\mathrm{L}}=1 \mathrm{k} \Omega$, Figure 7 & & & 20 & $\mathrm{MHz}$ \\
\hline Off-Isolation & $V_{I S O}$ & $\begin{array}{l}V_{I N}, V_{I N A}, V_{\mid N B_{3}}=1 V_{R M S}, t=100 \mathrm{kHz} \\
R_{L}=50 \Omega, C_{L}=15 p F \text {, Figure } 8\end{array}$ & & 65 & & $\mathrm{~dB}$ \\
\hline Crosstalk & $\mathrm{V}_{\mathrm{CT}}$ & $R_{S}=R L=50 \Omega$, Figure 9 & & 62 & & $\mathrm{~dB}$ \\
\hline $\begin{array}{l}\text { Total Harmonic Distortion Plus } \\
\text { Noise }\end{array}$ & $\mathrm{THD}+\mathrm{N}$ & $R S=R L=1 \mathrm{k} \Omega, f=20 \mathrm{~Hz}$ to $20 \mathrm{kHz}$ & & 0.0014 & & $\%$ \\
\hline Charge Injection & Q & $\begin{array}{l}V_{I N}, V_{I N A_{2}}, V_{\mid N B_{-}}=G N D, C_{L}=1 n F, \\
\text { Figure } 10\end{array}$ & & 200 & & $\mathrm{pC}$ \\
\hline
\end{tabular}


MAX14752/MAX14753

8-Channel/Dual 4-Channel 72 V Analog Multiplexers

DC Electrical Characteristics-Single Supply

$\left(V_{D D}=+70 \mathrm{~V}, V_{S S}=V_{G N D}=0 \mathrm{~V}, V_{E N}=+3.3 \mathrm{~V}, \mathrm{~T}_{\mathrm{A}}=-40^{\circ} \mathrm{C}\right.$ to $+85^{\circ} \mathrm{C}$, unless otherwise noted. Typical values are at $\mathrm{T}_{\mathrm{A}}=+25^{\circ} \mathrm{C}$. $)($ Note 2)

\begin{tabular}{|c|c|c|c|c|c|c|c|}
\hline PARAMETER & SYMBOL & \multicolumn{2}{|c|}{ CONDITIONS } & MIN & TYP & MAX & UNITS \\
\hline On-Resistance & RON & \multicolumn{2}{|c|}{ 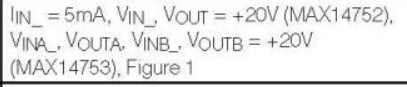 } & & 60 & 130 & \\
\hline \multirow{2}{*}{$\begin{array}{l}\text { OUT, OUTA, OUTB Off-Leakage } \\
\text { Current }\end{array}$} & \multirow{2}{*}{$\begin{array}{l}\text { lOUT(OFF), } \\
\text { lOUTA(OF), } \\
\text { lOUTB(OF) }\end{array}$} & \multicolumn{2}{|c|}{$\begin{array}{l}\text { MAX14752: } \mathrm{V} \text { OUT }=+40 \mathrm{~V}, \mathrm{~V}_{\mathrm{IN}}=\mathrm{V}_{\mathrm{IN} \mathrm{N}_{-}=}= \\
\mathrm{V}_{\mid \mathrm{IB}_{-}}=+10 \mathrm{~V}, \text { Figure } 3\end{array}$} & 20 & & +20 & \multirow{2}{*}{$\mathrm{nA}$} \\
\hline & & \multicolumn{2}{|c|}{$\begin{array}{l}\text { MAX14753: VouT }=+40 \mathrm{~V}, \mathrm{VIN}_{-}=\mathrm{V}_{\mid N_{\mathrm{N}}}= \\
\mathrm{V}_{\mathrm{INB}}=+10 \mathrm{~V}, \text { Figure } 3\end{array}$} & -10 & & +10 & \\
\hline \multirow{4}{*}{ On-Input Capacitance } & \multirow{4}{*}{ CIN_ON } & \multirow{2}{*}{$\begin{array}{l}\text { MAX } 14752, V_{D D}=+50 \mathrm{~V} \\
\text { OUT unconnected }\end{array}$} & $V_{1 N_{-}}=4 \mathrm{~V}$ & & 43 & & \multirow{4}{*}{$\mathrm{pF}$} \\
\hline & & & $V_{\mid N_{-}}=25 \mathrm{~V}$ & & 26 & & \\
\hline & & \multirow{2}{*}{$\begin{array}{l}\text { MAX } 14753, V_{D D}=+50 \mathrm{~V} \text {, } \\
\text { OUTA, OUTB } \\
\text { unconnected }\end{array}$} & $\begin{array}{l}V_{1 N A_{2}}, V_{I N B_{-}}= \\
4 \mathrm{~V}\end{array}$ & & 26 & & \\
\hline & & & $\begin{array}{l}V_{\text {INA_, }}, V_{\mid N B_{-}}= \\
25 \mathrm{~V}\end{array}$ & & 16 & & \\
\hline \multirow{4}{*}{ Off-Input Capacitance } & \multirow{4}{*}{ CIN_OFF } & \multirow{2}{*}{$M A X 14752, V_{D D}=+50 \mathrm{~V}$} & $V_{I N_{-}}=4 \mathrm{~V}$ & & 6 & & \multirow{4}{*}{$\mathrm{pF}$} \\
\hline & & & $V_{1 N_{-}}=25 \mathrm{~V}$ & & 3.7 & & \\
\hline & & \multirow{2}{*}{$M A X+4753, V_{D D}=+50 \mathrm{~V}$} & $\begin{array}{l}V_{I N A_{2},} V_{I N B_{-}}= \\
4 \mathrm{~V}\end{array}$ & & 6 & & \\
\hline & & & 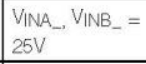 & & 3.7 & & \\
\hline \multirow{4}{*}{ Off-Output Capacitance } & \multirow{4}{*}{ COUT_OFF } & \multirow{2}{*}{$M A X 14752, V_{D D}=+50 \mathrm{~V}$} & $V_{\text {OUT_ }}=4 \mathrm{~V}$ & & 35 & & \multirow{4}{*}{$\mathrm{pF}$} \\
\hline & & & VOUT_ $=25 \mathrm{~V}$ & & 20 & & \\
\hline & & \multirow{2}{*}{$M A X 14753, V_{D D}=+50 \mathrm{~V}$} & $\begin{array}{l}\text { VouTA, } \\
\text { VouTB_ }=4 \mathrm{~V} \\
\end{array}$ & & 19 & & \\
\hline & & & $\begin{array}{l}\text { VOUTA. } \\
\text { VOUTB_ }=25 \mathrm{~V}\end{array}$ & & 11 & & \\
\hline
\end{tabular}

Note 2: All parameters in single-supply operation are expected to be the same as in dual-supplies operation. Note 3: IN-OUT capacitances are negligible $(<1 \mathrm{pF})$ 
Test Circuits/Timing Diagrams/Truth Tables
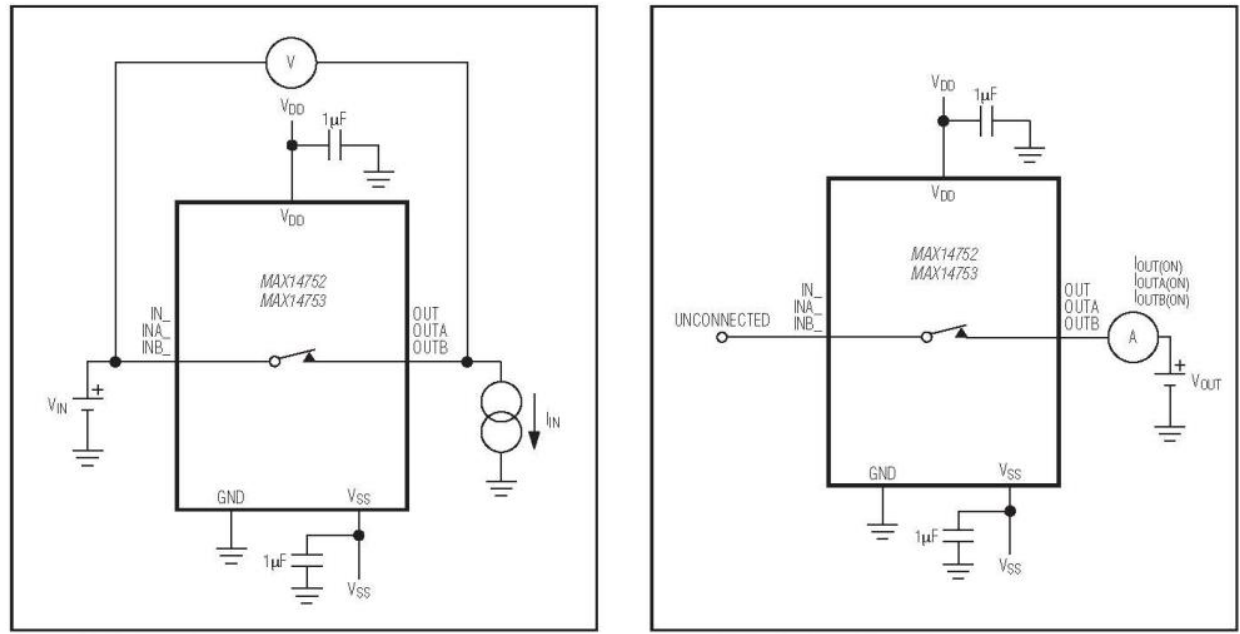

Figure 1. On-Resistance

Figure 2. On-Leakage Current

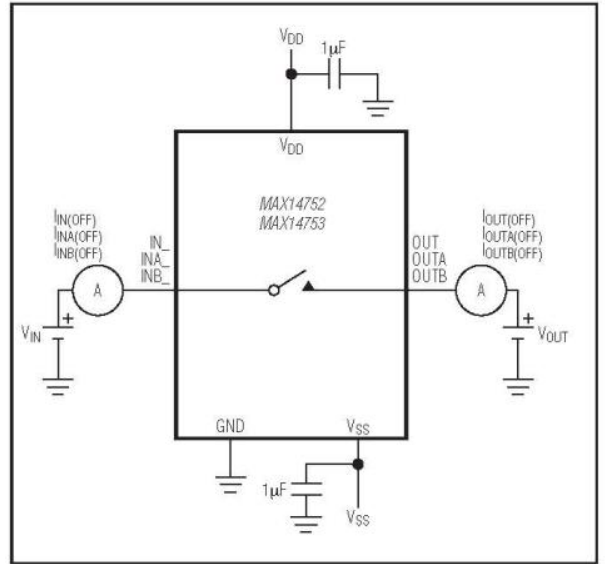

Figure 3. Off-Leakage Current 
Test Circuits/Timing Diagrams/Truth Tables (continued)

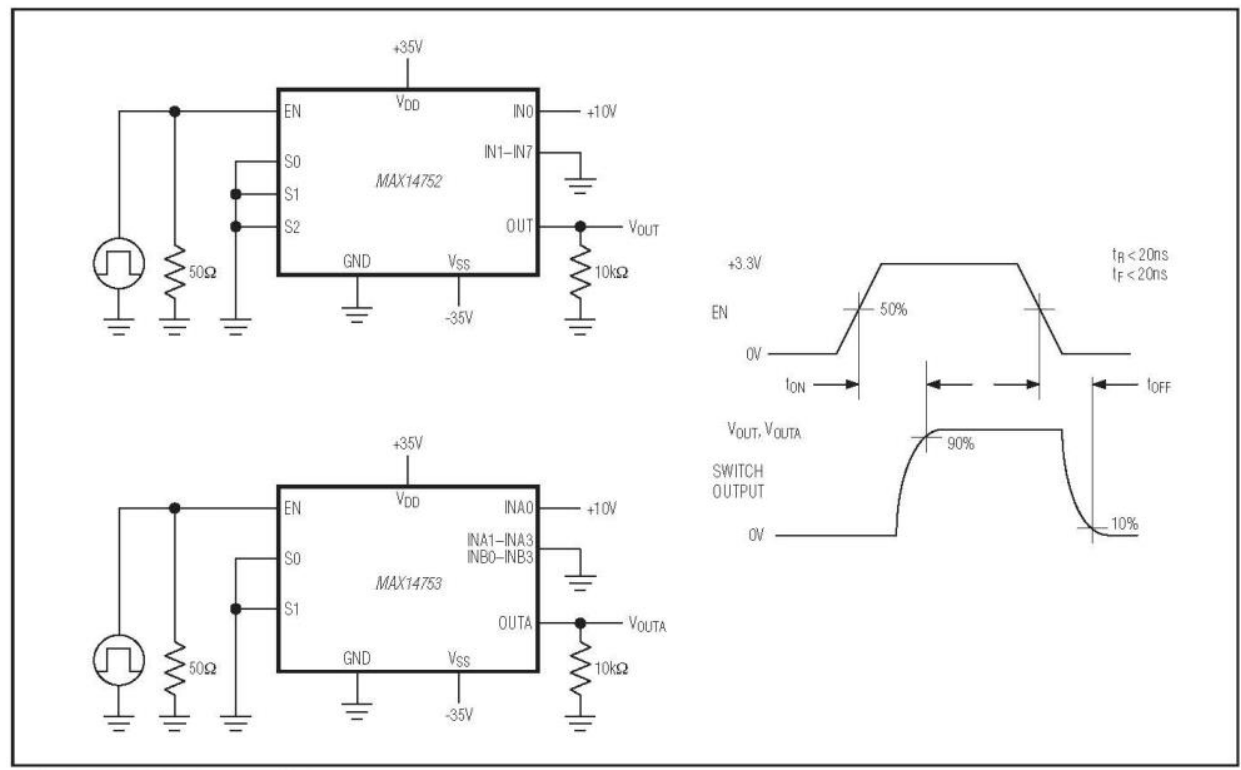

Figure 4. Enable Switching Time 
Test Circuits/Timing Diagrams/Truth Tables (continued)
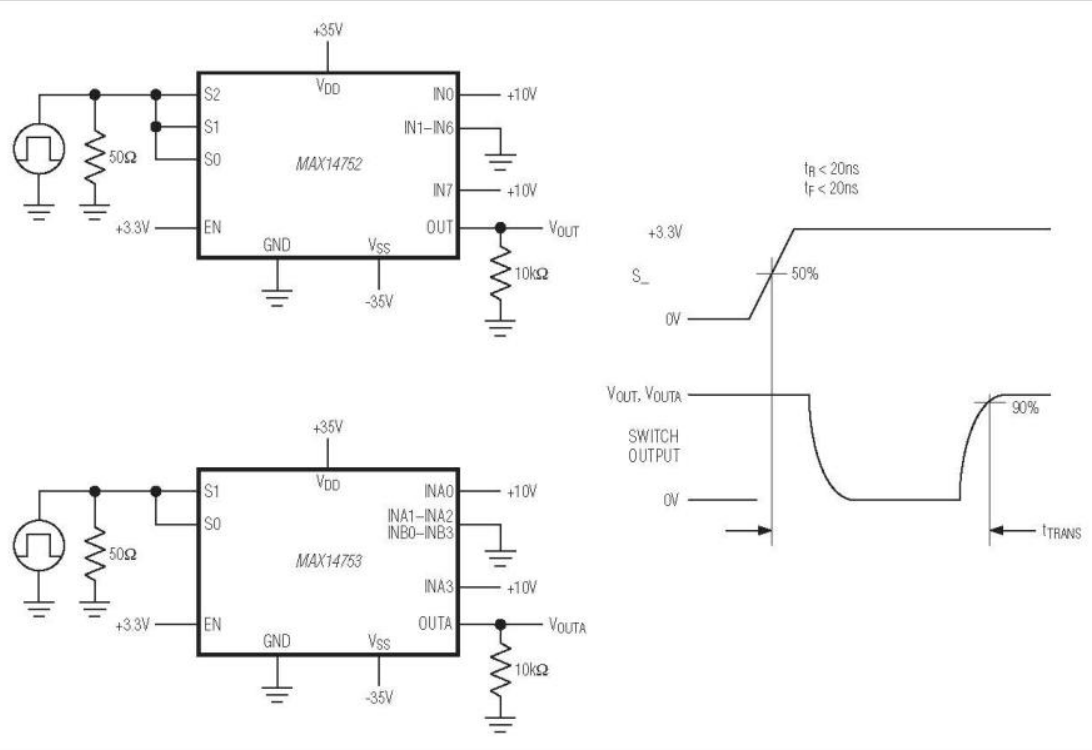

Figure 5. Transition Time
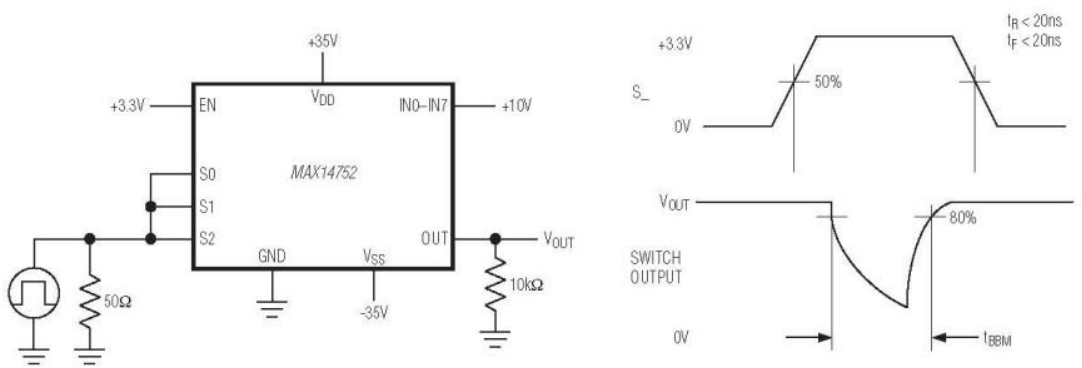

Figure 6. Break-Before-Make Interval 
Test Circuits/Timing Diagrams/Truth Tables (continued)

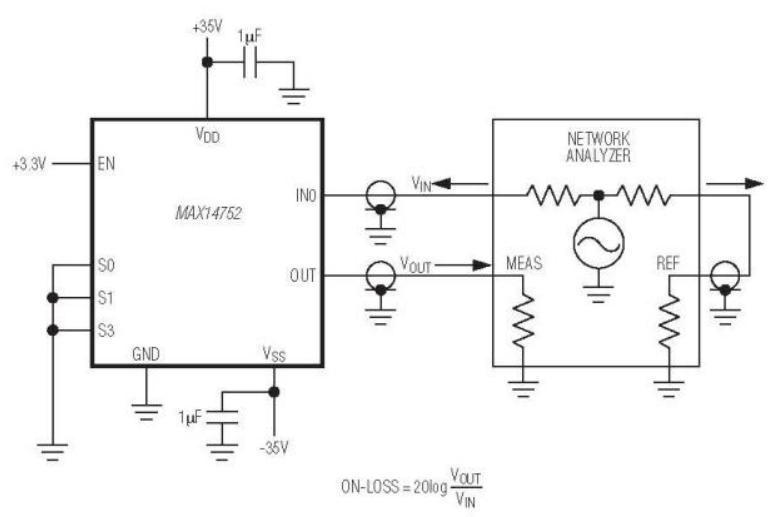

Figure 7. Frequency Response

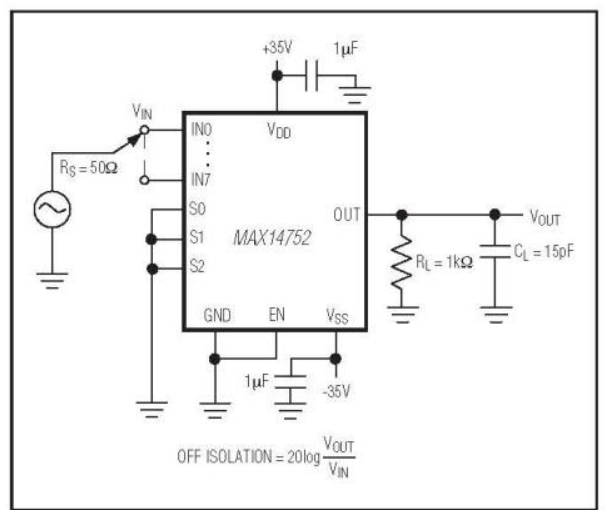

Figure 8. Off-isolation

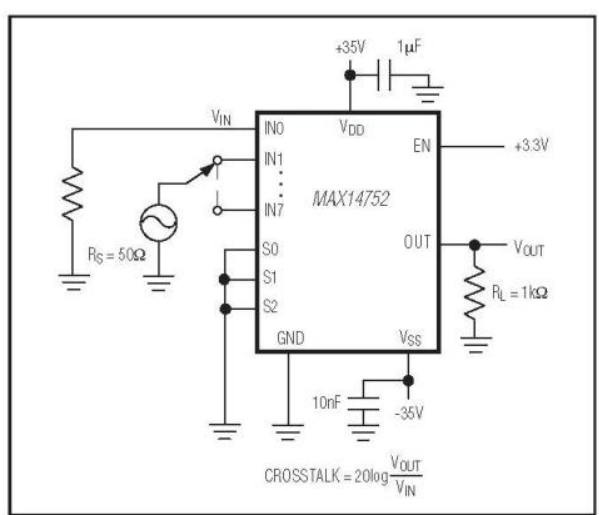

Figure 9. Crosstalk 
Test Circuits/Timing Diagrams/Truth Tables (continued)

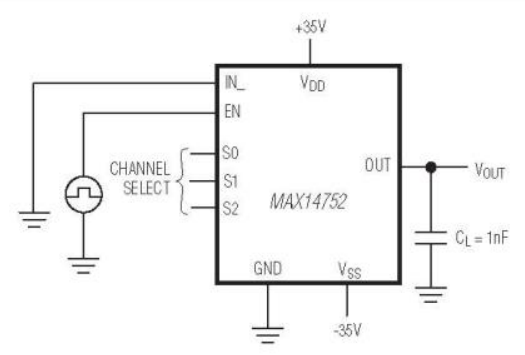

$V_{\text {EN }}$

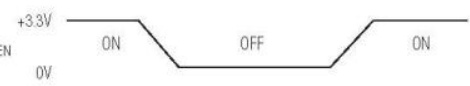

vour

ov $\tau^{\Delta V_{\text {OUT }}}$ $\triangle$ VoUT IS THE MEASURED VOLTAGE DUE TO CHARGE TRANSFER ERRORO WHEN THE CHANNEL TURNS OFF $0=C_{L} \times \Delta V_{\text {OUT }}$

Figure 10. Charge Injection

Table 1. MAX14752 Truth Table

\begin{tabular}{|c|c|c|c|c|}
\hline S2 & S1 & S0 & EN & OUT \\
\hline$X$ & $X$ & $X$ & 0 & All off \\
\hline 0 & 0 & 0 & 1 & IN0 \\
\hline 0 & 0 & 1 & 1 & IN1 \\
\hline 0 & 1 & 0 & 1 & IN2 \\
\hline 0 & 1 & 1 & 1 & IN3 \\
\hline 1 & 0 & 0 & 1 & IN4 \\
\hline 1 & 0 & 1 & 1 & IN5 \\
\hline 1 & 1 & 0 & 1 & IN6 \\
\hline 1 & 1 & 1 & 1 & IN7 \\
\hline
\end{tabular}

Table 2. MAX14753 Truth Table

\begin{tabular}{|c|c|c|c|c|}
\hline S1 & S0 & EN & OUTA & OUTB \\
\hline$X$ & $X$ & 0 & All off & All off \\
\hline 0 & 0 & 1 & INA0 & INB0 \\
\hline 0 & 1 & 1 & INA1 & INB1 \\
\hline 1 & 0 & 1 & INA2 & INB2 \\
\hline 1 & 1 & 1 & INA3 & INB3 \\
\hline
\end{tabular}


Typical Operating Characteristics

( $\mathrm{T}_{\mathrm{A}}=+25^{\circ} \mathrm{C}$, unless otherwise noted.)
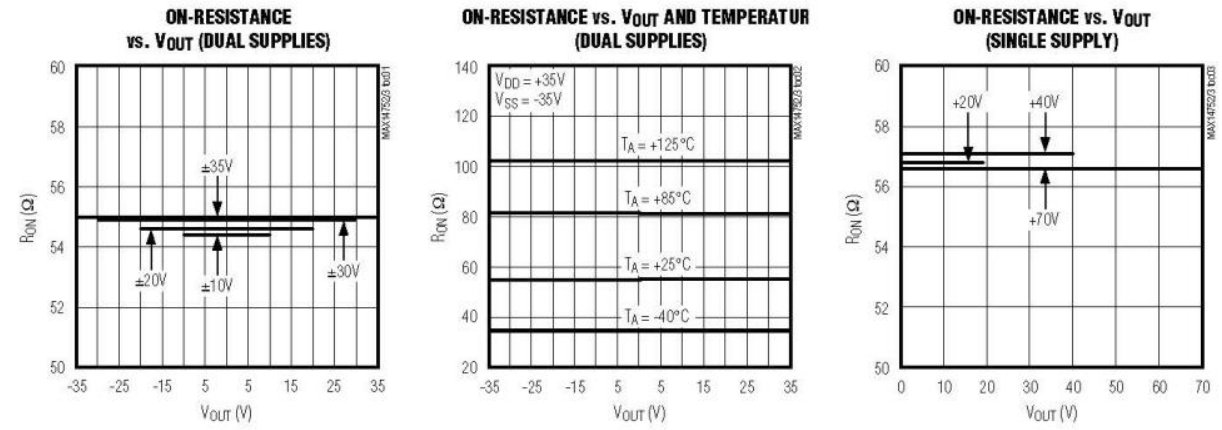

ON-RESISTANCE vs. VOUT AMD TEMPERATU
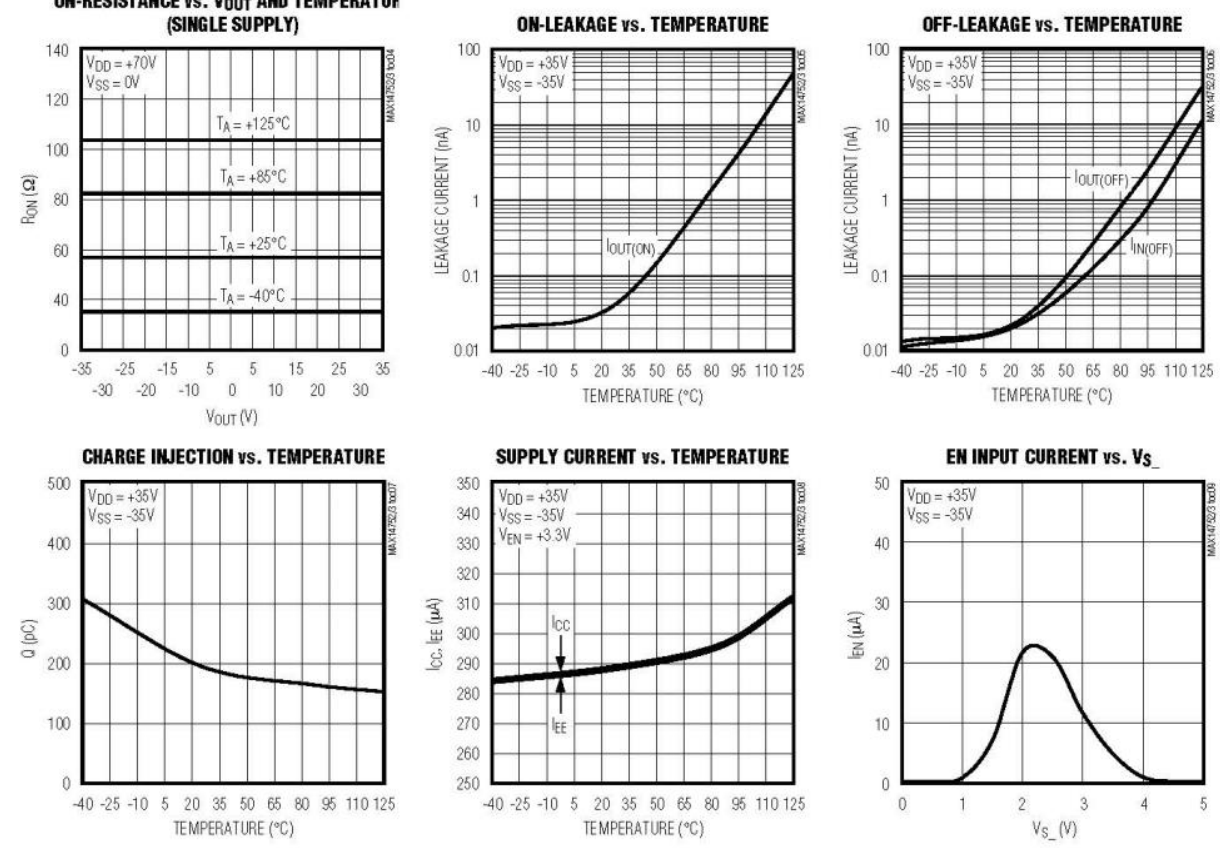
Typical Operating Characteristics (continued)

$\left(T_{A}=+25^{\circ} \mathrm{C}\right.$, unless otherwise noted. $)$

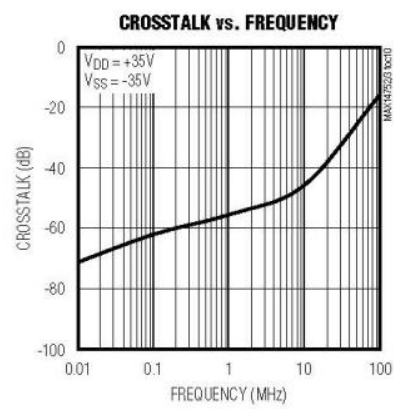

OFF-ISOLATION vS. FREQUENCY
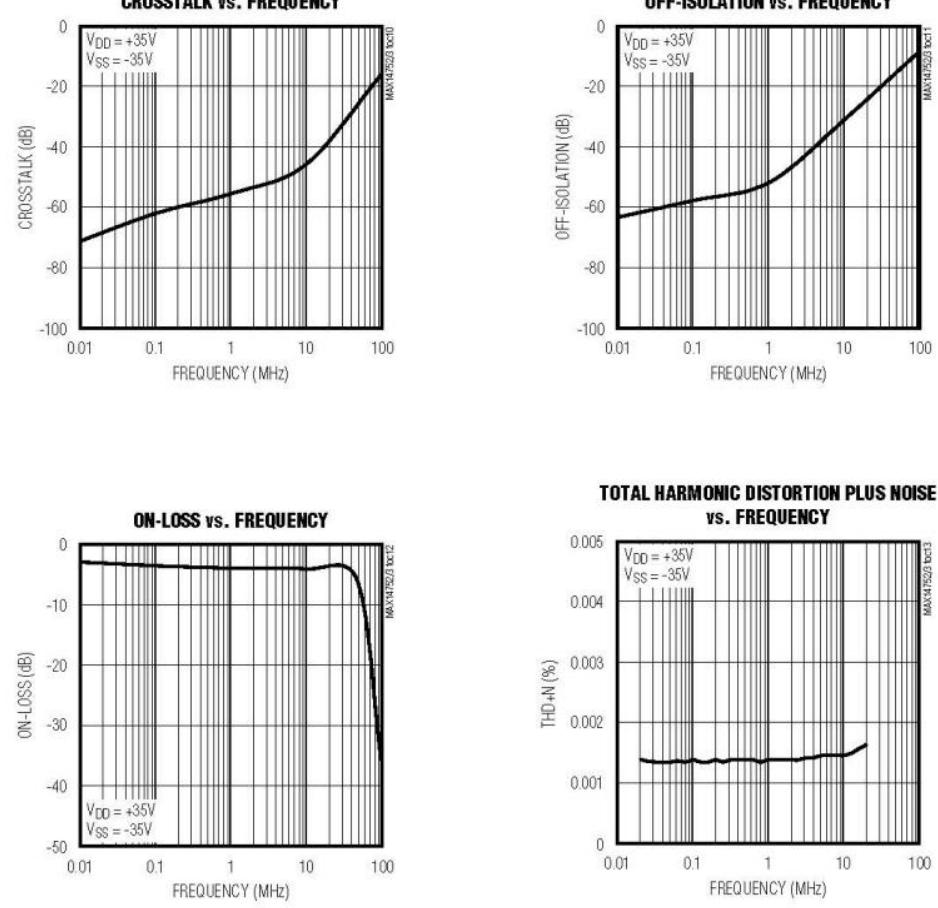
MAX14752/MAX14753

8-Channel/Dual 4-Channel $72 \mathrm{~V}$ Analog Multiplexers

MAX14752 Pin Description (Single 8-to-1 Mux)

\begin{tabular}{|c|c|c|}
\hline PIN & NAME & FUNCTION \\
\hline 1 & So & Mux Input Select \\
\hline 2 & EN & $\begin{array}{l}\text { Mux Enable. Drive EN high to enable the device. The EN high voltage defines input logic voltage level for } \\
\text { So, S1, and S2. }\end{array}$ \\
\hline 3 & $V_{S S}$ & Negative Supply Voltage. Bypass VSS to GND with a $1 \mu \mathrm{F}$ ceramic capacitor. \\
\hline 4 & INO & Bidirectional Analog Input \\
\hline 5 & IN1 & Bidirectional Analog Input \\
\hline 6 & IN2 & Bidirectional Analog Input \\
\hline 7 & IN3 & Bidirectional Analog Input \\
\hline 8 & OUT & Bidirectional Analog Output \\
\hline 9 & IN7 & Bidirectional Analog Input \\
\hline 10 & IN6 & Bidirectional Analog Input \\
\hline 11 & IN5 & Bidirectional Analog Input \\
\hline 12 & IN4 & Bidirectional Analog Input \\
\hline 13 & VDD & Positive Supply Voltage. Bypass VDD to GND with a $1 \mu \mathrm{F}$ ceramic capacitor. \\
\hline 14 & GND & $\begin{array}{l}\text { Ground. Connect GND to VSS for single supply. Bypass GND to VSS with a } 1 \mu \mathrm{F} \text { ceramic capacitor for dual } \\
\text { supply. }\end{array}$ \\
\hline 15 & S2 & Mux Input Select \\
\hline 16 & $\mathrm{~S} 1$ & Mux Input Select \\
\hline
\end{tabular}

\section{MAX14753 Pin Description (Dual 4-to-1 Mux)}

\begin{tabular}{|c|c|c|}
\hline PIN & NAME & FUNCTION \\
\hline 1 & So & Mux Input Select \\
\hline 2 & EN & $\begin{array}{l}\text { Mux Enable. Drive EN high to enable the device. The EN high voltage defines input logic voltage level for } \\
\text { So and S1. }\end{array}$ \\
\hline 3 & $V_{S S}$ & Negative Supply Voltage. Bypass VSS to GND with a $1 \mu \mathrm{F}$ ceramic capacitor. \\
\hline 4 & INAO & Bidirectional Analog Input \\
\hline 5 & INA1 & Bidirectional Analog Input \\
\hline 6 & INA2 & Bidirectional Analog Input \\
\hline 7 & INA3 & Bidirectional Analog Input \\
\hline 8 & OUTA & Bidirectional Analog Output \\
\hline 9 & OUTB & Bidirectional Analog Output \\
\hline 10 & INB3 & Bidirectional Analog Input \\
\hline 11 & INB2 & Bidirectional Analog Input \\
\hline 12 & INB1 & Bidirectional Analog Input \\
\hline 13 & INBO & Bidirectional Analog Input \\
\hline 14 & $V_{D D}$ & Positive Supply Voltage. Bypass VDD to GND with a $1 \mu \mathrm{F}$ ceramic capacitor. \\
\hline 15 & GND & $\begin{array}{l}\text { Ground. Connect GND to VSS for single supply. Bypass GND to VSS with a } 1 \mu \mathrm{F} \text { ceramic capacitor for dual } \\
\text { supply. }\end{array}$ \\
\hline 16 & $\mathrm{~S} 1$ & Mux Input Select \\
\hline
\end{tabular}




\section{Detailed Description}

The MAX14752/MAX14753 are 8-to-1 and dual 4-to-1 high-voltage analog multiplexers. Both devices feature $60 \Omega$ (typ) on-resistance with $0.03 \Omega$ (typ) on-resistance flatness. These low on-resistance multiplexers conduct equally well in either direction.

The MAX14752 is an 8-to-1 multiplexer and MAX14753 is a dual 4-to-1 multiplexer. Both devices operate with dual supplies of $\pm 10 \mathrm{~V}$ to $\pm 36 \mathrm{~V}$ or a single supply of $+20 \mathrm{~V}$ to $+72 \mathrm{~V}$. Both devices can also operate with unbalanced supplies, such as $+36 \mathrm{~V}$ and $-10 \mathrm{~V}$. These multiplexers support rail-to-rail input and output signals. The control logic level is defined via the EN input. These devices do not require power-supply sequencing.

\section{Applications Information}

\section{Current Through the Mux}

The current flowing through each on-channel of the MAX14752/MAX14753 multiplexers must be limited to $\pm 5 \mathrm{~mA}$ for normal operation. If the current exceeds this limit, an internal leakage current from that channel to Vss appears. Larger input current does not destroy the device if the max power dissipation is not exceeded

\section{Input Voltage Clamping}

For applications that require input voltages beyond the normal operating voltages, the internal input diodes to $V_{D D}$ and $V_{S S}$ can be used to limit the input voltages. As shown in Figure 11, series resistors can be employed at the inputs to limit the currents flowing into the diodes during undervoltage and overvoltage conditions. Choose the limiting resistors such that the input currents are limited to IIN $(\max )=100 \mathrm{~mA}$. The values of the current limit resistors can be calculated as the larger of RLIM+ and RLIM-

$$
\begin{aligned}
& R_{L_{I M+}}=\frac{V_{I_{N}}(\max )-V_{D D}}{h_{N}(\max )} \\
& R_{\text {LIM }^{-}}=\frac{V_{S S}-V_{I_{N}}(\min )}{l_{N_{N}}(\max )}
\end{aligned}
$$

During an undervoltage or overvoltage condition, the input impedance is equal to RபM. The additional power dissipation due to the fault currents needs to be calculated. The MAX14752/MAX14753 multiplexer operates normally on a channel that is on during an overvoltage or undervoltage clamping condition on a second channel that is not switched.

\section{Beyond-the-Rail Input}

If input voltages are expected to go beyond the supply voltages, but within the absolute maximum supply voltages of the MAX14752/MAX14753, add two diodes in series with the supplies as shown in Figure 12.

During undervoltage and overvoltage events, the internal diodes pull VDD/VSs supplies up/down. An advantage of this scheme is that the input impedance is high and currents do not flow through the MAX14752/MAX14753 during overvoltage and undervoltage events. The input voltages must be limited to the voltages specified in the Absolute Maximum Ratings section.

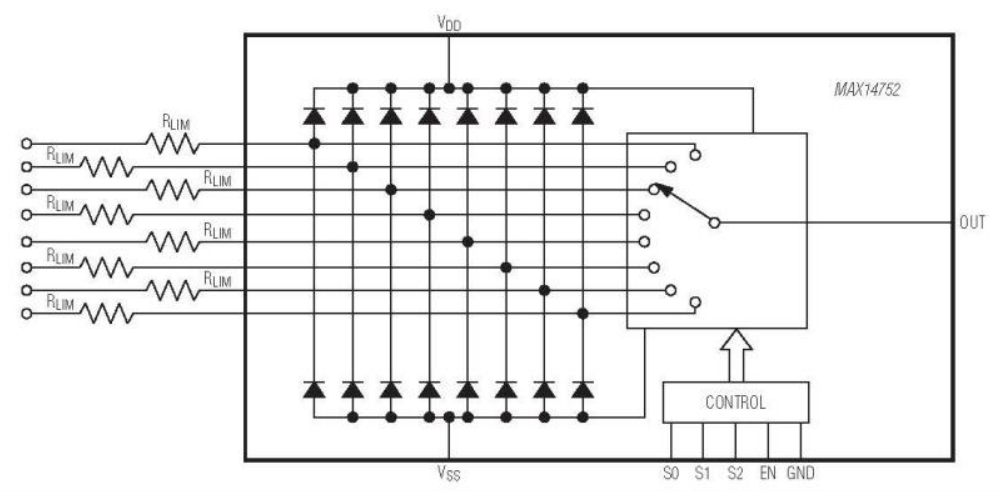

Figure 11. input Overvoltage and Undervoltage Clamping 


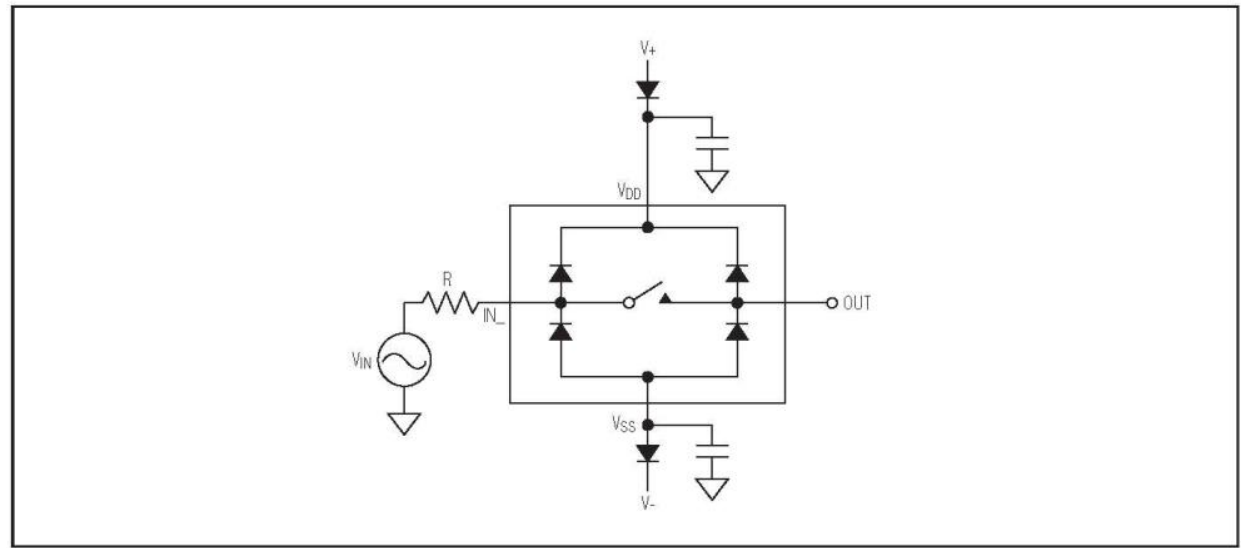

Figure 12. Beyond-the-Rail Application

\section{Pin Configurations}

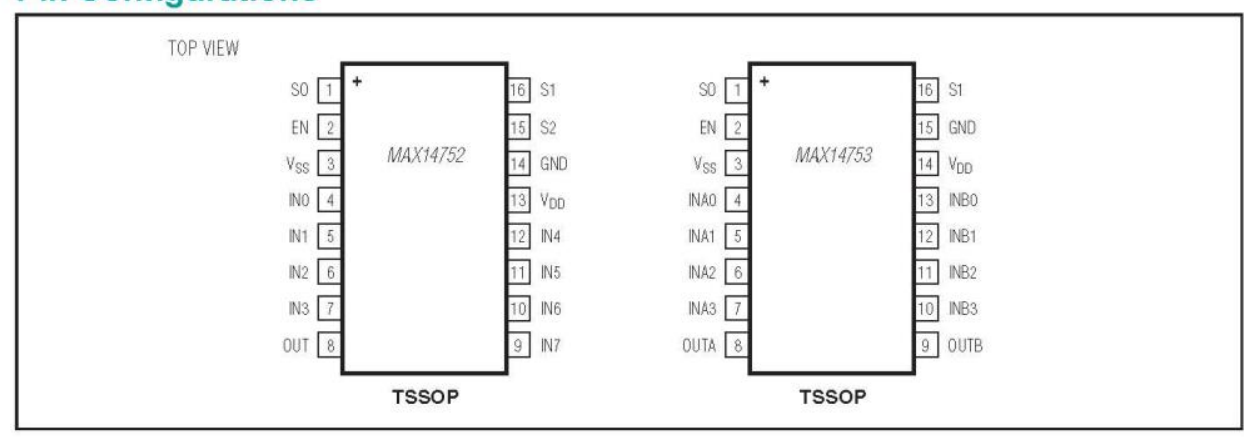

Chip Information

PROCESS: CMOS

\section{Package Information}

For the latest package outline information and land patterns (footprints), go to www.maximintegrated.com/packages. Note that a "+", "\#", or "-" in the package code indicates RoHS status only Package drawings may show a different suffix character, but the drawing pertains to the package regardless of RoHS status.

\begin{tabular}{|c|c|c|c|}
\hline $\begin{array}{c}\text { PACKAGE } \\
\text { TYPE }\end{array}$ & $\begin{array}{c}\text { PACKAGE } \\
\text { CODE }\end{array}$ & $\begin{array}{c}\text { OUTLINE } \\
\text { NO. }\end{array}$ & $\begin{array}{c}\text { LAND } \\
\text { PATTERN NO. }\end{array}$ \\
\hline 16 TSSOP & $\mathrm{U} 16+1$ & $\underline{\mathbf{2 1 - 0 0 6 6}}$ & $\underline{\mathbf{9 0 - 0 1 1 7}}$ \\
\hline
\end{tabular}


MAX14752/MAX14753

8-Channel/Dual 4-Channel $72 \mathrm{~V}$ Analog Multiplexers

\section{Revision History}

\begin{tabular}{|c|c|l|c|}
\hline $\begin{array}{c}\text { REVISION } \\
\text { NUMBER }\end{array}$ & $\begin{array}{c}\text { REVISION } \\
\text { DATE }\end{array}$ & \multicolumn{1}{|c|}{ DESCRIPTION } & $\begin{array}{c}\text { PAGES } \\
\text { CHANGED }\end{array}$ \\
\hline 0 & $8 / 08$ & Initial release & - \\
\hline 1 & $10 / 08$ & $\begin{array}{l}\text { Changed the units from mA to } \mu \text { A for VDD and VSS supply current in the DC Electrical } \\
\text { Characteristics_Dual Supplies table }\end{array}$ & 2 \\
\hline 2 & $2 / 09$ & Added capacitance information to EC table & $\begin{array}{c}2,4,13,14, \\
15,16\end{array}$ \\
\hline 3 & $7 / 10$ & $\begin{array}{l}\text { Deleted the "Input Capacitance" parameter from the DC Electrical Characteristics- } \\
\text { Dual Supplies }\end{array}$ & 3 \\
\hline 4 & $4 / 15$ & Removed automotive reference & 1 \\
\hline 5 & $5 / 15$ & Revised Benefits and Features section & 1 \\
\hline
\end{tabular}

For pricing, delivery, and ordering information, please contact Maxim Direct at 1-888-629-4642, or visit Maxim Integrated's website at www.maximintegrated.com.

Maxim Integrated cannot assume responsibility for use of any circuitry other than circuitry entirely embodied in a Maxim integrated product. No circuit patent Hicenses are implied. Maxim integrated reserves the right to change the circuitry and specifications without notice at any time. The parametric values (min and max limits) shown in the Electrical Characteristics table are guaranteed. Other parametric values quoted in this data sheet are provided for guidance.

Maxim Integrated and the Maxim Integrated logo are trademarks of Maxim Integrated Products, Inc

(2) 2015 Maxim Integrated Products, Inc. | 15 